\title{
Understanding the Experiences of Justice-Involved Youth at the Intersection of the Criminal Justice and Mental Health Systems
}

\author{
by
}

Elisabeth Leroux

A thesis submitted to the Faculty of Graduate and Postdoctoral Affairs in partial fulfillment of the requirements for the degree of

Doctor of Philosophy

in

Psychology

Carleton University

Ottawa, Ontario

(C) 2019

Elisabeth Leroux 


\begin{abstract}
In this dissertation, I examined the psychosocial effects of progressing through the youth criminal justice system. The studies presented here are tied together by the shared goal of promoting a welfare-based youth criminal justice system that adopts an extended responsibility of care toward justice-involved youth beyond reducing recidivism.

Study 1 examined how clinician-derived diagnoses and recommendations are determined in a forensic context when youth provide information about their mental health that is consistent, or inconsistent, with information provided by their caregivers. Clinicians weighed information from youth more heavily when making internalizingspectrum diagnoses such as mood or anxiety disorders, whereas information from caregivers was more heavily weighed when making externalizing-spectrum diagnoses such as conduct disorder, and clinical recommendations.

Study 2 examined changes in the mental health, self-esteem, and perceived social support, of justice-involved youth as they transitioned through the adjudication period of the youth criminal justice system process. Mental health and self-esteem were significantly different across time, with greater negative changes associated with longer adjudication times. Youth who committed sexual offences reported significantly greater negative changes in mental health and self-esteem compared to youth who committed non-sexual offences.
\end{abstract}

Using a mixed-methods approach, Study 3 examined if, and how, justice-involved youth experienced stigma associated with criminal justice system involvement (offending-based stigma). Justice-involved youth experienced moderate internalized offending-based stigma, with greater internalized stigma associated with greater mental 
health symptoms, and lower self-esteem. The qualitative findings indicated justiceinvolved youth construct themselves as devalued members of society because of their offending status. Using an intersectional lens, how youth took up their offending status depended on how they also took up their race, masculinity, and class.

This dissertation applied a broad inter-disciplinary lens, drawing on several fields of study, including correctional policy, criminogenic risk, developmental criminology, developmental psychopathology, forensic assessment, forensic mental health, human rights, intersectionality, legal discourse, and stigma. The conclusions presented in this dissertation build on the empirical foundation provided by these works to further demonstrate the youth criminal justice system in Canada must move away from a strict focus on reducing recidivism among justice-involved youth. 


\section{Acknowledgements}

First and foremost, I would like to acknowledge and thank the youth who participated in my dissertation studies. Their courage to speak with such candor and humour about the most difficult experiences of their lives is admirable. Their stories are a powerful reminder to never lose sight of the humanness of those in the criminal justice system. In the words of justice reformer Bryan Stevenson, "each of us is more than the worst thing we've ever done".

I would like to deeply thank my co-supervisor, Dr. Michael Seto, for everything he has done to support me to date. No one has had a more profound impact on my academic life than Michael. I first emailed Michael in the $3^{\text {rd }}$ year of my undergraduate degree, having no idea of his reputation, or status as a leading expert in the field of sexual offending. I'm not sure what prompted him to respond to that fateful email and take me on as a student, but the academic Gods were surely shining on me that day. Michael's mentorship over the past eight years has shaped me into the academic I am today, and shaped the trajectory of my career, for which I will be forever grateful. I look forward to more years of him telling me how to live my life - which I know is one of his favourite things to do!

I would also like to thank my co-supervisor, Dr. Kevin Nunes, from Carleton. His willingness to supervise me enabled me to pursue the degree of my choice. I am very grateful for the doors Kevin has opened for me. I would also like to thank Dr. Tracey Skilling for her constant support throughout this project. Tracey's guidance over the past few years has been invaluable to the success of this work. I am also very appreciative to have a such a strong and empowered female mentor to look up to. 
I would like to acknowledge my committee members, Dr. Shelley Brown and Dr. Andrea Howard. I am very thankful for their flexibility in accommodating the shifting and changing nature of my dissertation work. I would like to thank Dr. Lara Karaian, for her willingness to act as my internal examiner. I would also like to thank Dr. Michele Peterson-Badali for agreeing to be my external examiner. I have always admired Dr. Peterson-Badali's work, and it is such a privilege to have her evaluating my doctoral dissertation.

In addition, I would like to thank the University Medical Research Fund (UMRF), who provided the funding necessary to complete this work.

Finally, I am immensely grateful for my family: my mother, whose editorial comments during my formative writing years rivaled those of Reviewer 2 but undoubtedly made me into the writer I am now; my father, who taught me to be critical of everything, and whose voice I always hear saying "show me the data"; my brother, who despite our sometimes vicious sibling rivalry, has always been there to witness my life; my partner, who has unconditionally supported me since the day we met; and last, but certainly not least, Huron the cat, who has been my best friend throughout this whole process. I love you all. 


\section{Dedication}

I dedicate this dissertation to everyone who could not be here. To my GrandmaJean, whose quiet tenacity was an inspiration. It took her almost 20 years but she proudly graduated from Carleton University with a Bachelor degree in 1970. My childhood memories will also always include pictures of her diligently working on completing my grandfather's unfinished book. To my Uncle Paul, who was a great friend. If only we could laugh and share celebratory egg rolls one more time. To my Uncle Kurt, who was a quintessential academic, and lived out his academic passion every day. Finally, to my grandfather Poppa, whose decision to attend Carleton University after the war changed the trajectory of his life, and in so doing brought me here today. I am proud to dedicated this work to all of you. 


\section{Table of Contents}

Chapter 1: Introduction 1

Rationale for This Dissertation 3

Dissertation Summary

Chapter 2: The Effect of Youth-Caregiver Discrepancies in Forensic 13 Assessment Clinician Decision-Making

$\begin{array}{ll}\text { Informant Discrepancies } & 13\end{array}$

$\begin{array}{ll}\text { Informant Reliability in the Forensic Context } & 17\end{array}$

$\begin{array}{ll}\text { Current Study } & 19\end{array}$

$\begin{array}{ll}\text { Methods } & 22\end{array}$

Participants $\quad 22$

$\begin{array}{ll}\text { Measures } & 26\end{array}$

Youth Self-Report and Child Behavior Checklist 26

Clinician-derived outcomes 28

Diagnoses 28

Recommendations 29

Data Analysis Plan 30

$\begin{array}{ll}\text { Results } & 32\end{array}$

Clinician-Derived Diagnoses 33

Externalizing spectrum 33

$\begin{array}{ll}\text { Internalizing spectrum } & 37\end{array}$

Clinician-Derived Recommendations 38

General mental health counselling $\quad 40$

$\begin{array}{ll}\text { Psychiatric medication } & 40\end{array}$

Anger management $\quad 40$

Custody recommendation $\quad 41$

Educational supports $\quad 41$

Discussion $\quad 42$

Clinician-Derived Diagnoses 43

Clinician-Derived Recommendations $\quad 45$ 
Limitations and Future Directions $\quad 49$

Chapter Bridge: From Post-Adjudication Forensic Assessments to Pre-Post 53 Adjudication Changes in Mental Health

Study 1 Summary 53

Study 2 Bridge $\quad 55$

Prioritizing differences in offence type $\quad 56$

Chapter 3: Changes in Mental Health, Self-Esteem, and Social Support 58

Among Justice-Involved Youth

Mental Health and Youth Offending 58

Shared risk explanation $\quad 59$

$\begin{array}{ll}\text { Causal mechanism } & 61\end{array}$

Exacerbation Effect of the Criminal Justice System 63

$\begin{array}{ll}\text { Current Study } & 65\end{array}$

$\begin{array}{ll}\text { Hypotheses } & 68\end{array}$

$\begin{array}{ll}\text { Methods } & 69\end{array}$

$\begin{array}{ll}\text { Participants } & 69\end{array}$

$\begin{array}{ll}\text { Recruitment } & 70\end{array}$

Sample descriptives $\quad 71$

$\begin{array}{ll}\text { Measures } & 75\end{array}$

Summary sheet $\quad 75$

Background information $\quad 76$

Revised Child and Anxiety and Depression Scale $\quad 77$

Rosenberg Self-Esteem Scale $\quad 78$

Multidimensional Scale of Perceived Social Support $\quad 78$

$\begin{array}{ll}\text { Procedure } & 79\end{array}$

Results $\quad 81$

Anxiety $\quad 83$

Depression $\quad 85$

Self-Esteem $\quad 89$

Social Support 90

$\begin{array}{ll}\text { Length of Time Since First Offence } & 91\end{array}$ 
$\begin{array}{ll}\text { Discussion } & 94\end{array}$

Sexual Versus Non-Sexual Offence Type 96

Etiological implications $\quad 97$

$\begin{array}{ll}\text { Policy Implications } & 100\end{array}$

Limitations and Future Directions 102

$\begin{array}{ll}\text { Conclusion } & 106\end{array}$

Chapter Bridge: From Pre-Post Adjudication Changes in Mental Health to 108

Internalized Offending-Based Stigma

$\begin{array}{ll}\text { Study } 2 \text { Summary } & 108\end{array}$

Prioritizing differences in offence type 108

Chapter 4: An Interdisciplinary Mixed-Method Analysis of Offending- 110

Based Stigma Among Justice-Involved Youth

$\begin{array}{ll}\text { Conceptual Considerations for Stigma Research } & 111\end{array}$

$\begin{array}{ll}\text { Social psychology framework of stigma } & 111\end{array}$

$\begin{array}{ll}\text { Sociological framework of stigma } & 113\end{array}$

$\begin{array}{ll}\text { Current Study } & 115\end{array}$

$\begin{array}{ll}\text { Methods } & 116\end{array}$

$\begin{array}{ll}\text { Participants } & 116\end{array}$

$\begin{array}{ll}\text { Measures } & 117\end{array}$

Internalized Stigma Scale of Offending 117

Qualitative interview 118

$\begin{array}{ll}\text { Procedure } & 120\end{array}$

$\begin{array}{lr}\text { Results } & 120\end{array}$

$\begin{array}{ll}\text { Quantitative Analysis } & 120\end{array}$

$\begin{array}{ll}\text { ISSO psychometric properties } & 120\end{array}$

Degree of internalized stigma $\quad 122$

ISSO post-hoc testing $\quad 125$

Association of offending-based stigma with psychosocial 125 variables

Qualitative Analysis

$\begin{array}{ll}\text { Theoretical approach } & 127\end{array}$ 
Analysis anchor points $\quad 129$

Analytical approach 133

Devalued social identity $\quad 135$

Restricted upward mobility $\quad 138$

Restricted masculinity $\quad 140$

$\begin{array}{ll}\text { Discussion } & 142\end{array}$

$\begin{array}{ll}\text { Conclusion } & 149\end{array}$

Chapter 5: Discussion $\quad 150$

$\begin{array}{ll}\text { Future Directions } & 151\end{array}$

$\begin{array}{ll}\text { References } & 156\end{array}$ 


\section{List of Tables}

\section{Chapter 2: The Effect of Youth-Caregiver Discrepancies in Forensic}

\section{Assessment Clinician Decision-Making}

Table 1 Descriptive information for full sample of youth included in 25 paired dyads

Table 2 Descriptive Statistics of YSR/CBCL Sub-Scales

Table 3 Clinician-Derived Diagnosis Regression Results 36

Table $4 \quad$ Clinician-Derived Recommendation Regression Results 39

Chapter 3: Changes in Mental Health, Self-Esteem, and Social Support

\section{Among Justice-Involved Youth}

Table 5 Descriptive information by offence type

Table 6

Reported Changes in Anxiety, Depression, Self-Esteem,

and Social Support, Pre- and Post-Charge

Table 7

Regression Results of Time Since Index Offence Charge

Predicting Changes in Reported Anxiety, Depression, and Self-Esteem

\section{Chapter 4: An Interdisciplinary Mixed-Method Analysis of Offending-}

\section{Based Stigma Among Justice-Involved Youth}

Table 8 Correlation matrix for Internalized Stigma Scale of

Offending total score sub scales

Table 9 Independent Samples T-Test Comparisons of

Internalized Stigma Scale of Offending

Table 10 Correlation Matrix for Internalized Stigma Scale of 


\section{List of Figures}

\section{Chapter 2: The Effect of Youth-Caregiver Discrepancies in Forensic Assessment Clinician Decision-Making}

Figure 1 Probability of Receiving a Conduct Disorder Diagnosis at Low and High Levels of Youth and Caregiver Reports of Rule-Breaking Behaviours

Chapter 3: Changes in Mental Health, Self-Esteem, and Social Support Among Justice-Involved Youth

Figure 2 Pre- and Post-Charge Mean Reported Anxiety Scores for Youth Who Committed Sexual Offences and Youth Who Committed Non-Sexual Offences.

Figure 3 Pre- and Post-Charge Mean Reported Depression Scores for Youth Who Committed Sexual Offences and Youth Who Committed Non-Sexual Offences.

Figure 4 Pre- and Post-Charge Mean Reported Self-Esteem Scores for Youth Who Committed Sexual Offences and Youth Who Committed Non-Sexual Offences. 


\section{List of Appendices}

Chapter 2: The Effect of Youth-Caregiver Discrepancies in Forensic Assessment Clinician Decision-Making

Appendix A Sample Copy of Youth Self-Report

Appendix B Sample Copy of Child Behavior Checklist

Chapter 3: Changes in Mental Health, Self-Esteem, and Social Support Among Justice-Involved Youth

Appendix C Summary Sheet

Appendix D Summary Sheet Coding Form

Appendix E Background Survey

Appendix F Modified Revised Child and Adolescent

Depression Scale - Short Version

Appendix G Modified Rosenberg Self-Esteem Scale

Appendix H Modified Multidimensional Scale of Perceived Social Support for Adolescents

Appendix I Centre for Addiction and Mental Health Consent Forms

Appendix J The Royal Ottawa Consent Forms

Appendix K Confirmation of Compensation

Appendix L Centre for Addiction and Mental Health Participant Debrief Form

Appendix M The Royal Ottawa Participant Debrief Form

Chapter 4: An Interdisciplinary Mixed-Method Analysis of OffendingBased Stigma Among Justice-Involved Youth

Appendix N Internalized Stigma Scale of Offending

Appendix O Combined Interview Protocol- Revised Adolescent 


\section{Chapter 1: Introduction}

This dissertation presents three studies that together represent a program of research aimed at advancing our understanding of the psychosocial effects of progressing through the youth criminal justice system. In this dissertation, there is no single overarching research question, but a collection of studies each located in a different literature and answering a distinct but related research question. The common thread among these studies, and the foundation of this program of research more broadly, is the prioritization of the youth experience with the justice system. Each study included here is premised on the position that the youth criminal justice system has an implicit responsibility of care toward justice-involved youth that extends beyond reducing recidivism. As such, this dissertation presents research explicitly oriented toward promoting a welfare-based youth criminal justice system. This is not to say that individual actors in the youth criminal justice system do not consider the welfare of justice-involved youth. But within the structural institution that is the youth criminal justice system, the explicit priority is set to be the maintenance of public safety, sometimes at the expense of the welfare of some of society's most vulnerable youth. It is because the expressed orientation of this dissertation breaks with traditionally prioritized risk-based research that makes it an important contribution to the discipline.

This dissertation also breaks with traditional forensic psychology approaches in the sense that it straddles the boundary between positivism and social constructionism. Meaning, while the work presented here sits within a positivist framework, there is clear reference to social constructionism throughout, most notably in how discussions related to social discourses are weaved in. Singer and Hunter (1999, p. 66) describe social 
discourses as "pieces of conversation or talk with an agenda," meaning language operates within a specific historical and socio-political context to construct knowledge. In this way, language does not simply mirror reality but plays a role in creating the meaning we attribute to experience (Fairclough, 2013). A recurring theme in this dissertation is how social discourses regarding sexual offending (e.g., "predatory," “deviant," "disgusting”) construct youth who have committed a sexual offence in particular ways, which in turn contributes to how they experience the youth criminal justice system.

Similarly, while not an explicitly critical dissertation, the work presented here is informed by critical theory which is concerned with changing society by interrogating the role of social power in creating and/or shaping social phenomena (Fairclough, 2013; How, 2003; Richardson, 2007). For example, the framing of this dissertation centers around the acknowledgment that the youth criminal justice system is a site of structural power and thereby serves to marginalize and oppress those who commit criminal offences. Although Studies 1 and 2 (described below) do not incorporate a critical lens per se, the rationale for pursuing these studies is nonetheless premised on the position that systemic change must take place within the youth criminal justice system due to existing power imbalances. Overall, the broad critical orientation of this dissertation characterizes it as actively oppositional to oppressive social power structures, which in turn introduces social and political ethics (i.e., what is thought to be right or wrong) into the work (Gellers, 2015). This must be acknowledged to appropriately locate the work within psychology research. Further, acknowledging these positions ultimately enhances the ethical integrity of this dissertation. 


\section{Rationale for This Dissertation}

Forensic psychology research has evolved directly from, and largely reflects, the priorities of the criminal justice system. In the current socio-political context, the criminal justice system is positioned and advertised as a system of reform for individuals. The language of "correctional system" and "rehabilitation" dominate our social discourses regarding the criminal justice system, reflecting the ethos that individuals who criminally offend require curative measures toward socially acceptable behaviour. The youth criminal justice system is even more so constructed and viewed as a system of rehabilitation because of how dominant discourses of childhood intersect with discourses of criminality. For example, the preamble of the Youth Criminal Justice Act (YCJA; 2002), the legal statute dictating the implementation of the Canadian youth justice system, explicitly states "society share (sic) a responsibility to address the developmental challenges and the needs of young persons...Canadian society should have a youth criminal justice system that...ensures accountability through meaningful consequences and effective rehabilitation and reintegration" (p.1). The preamble contributes to the construction of a legislative context that prioritizes rehabilitation due to the inherent vulnerability of youth. As discussed by Bala, Carrington, and Roberts (2009), the legal interpretation of the preamble is that public safety is a consequence of rehabilitation, which directs the activities of the system toward the facilitation of rehabilitation, instead of a reliance on punishment.

The political framing of the youth criminal justice system as a site of rehabilitation means the measure of success for the youth correctional system is defined primarily in terms of recidivism. Essentially, successful youth rehabilitation is equated 
with the absence of reoffending. Underpinning this program model is the criminogenic risk framework, which is premised on the assumption that youth who commit crimes possess risk factors that when addressed, presumably by the youth criminal justice system, will reduce future offending. A central concern within the criminogenic risk framework is the assessment of said risk.

Risk assessment has slowly dominated youth (and adult) criminal justice system policy and practice, expanding in scope from its original focus of assessing risk for recidivism to being used in policing, pre-trial processing, sentencing, and case management planning (Bechtel, Holsinger, Lowenkamp, \& Warren, 2017; Casey, Warren, \& Elek, 2011; Desmarais \& Singh, 2013; Jung \& Buro, 2017; Myburgh, Camman, \& Wormith, 2015; Nelson \& Vincent, 2018; Peterson-Badali, Skilling, \& Haqanee, 2015). This creep of the criminogenic risk framework into almost all areas of the criminal justice system has been critically interrogated elsewhere (e.g., Fitzgibbon, 2008; Gaes \& Bales, 2011; Hamilton, 2015; Hannah-Moffat, 2013; Kemshall, 2008; Maurutto \& Hannah-Moffat, 2006, 2007; McNeill, 2012), albeit predominantly from disciplines outside of forensic psychology. In contrast, within the discipline of forensic psychology, the dominant perspective is that a central goal of the field is to produce research that advances the propagation of the criminogenic risk framework, specifically the accurate assessment of risk (Andrews, Bonta, \& Wormith, 2011; Bonta \& Andrews, 2007; Monahan \& Skeem, 2016; Mossman, 2015; Polaschek, 2012). Unpacking the merits of this goal, and the inter- and intra-disciplinary debates regarding the criminogenic risk framework as a driving force of the criminal justice system, is beyond the scope of this dissertation. Rather, the point is to highlight that the goals of the 
discipline of forensic psychology have led to a youth justice research landscape dominated by studies conducted within a criminogenic risk framework.

The consequence of emphasizing research conducted within a criminogenic risk framework for the field of youth justice research is a saturation of studies focused on two main lines of inquiry: (1) What predicts entry into the criminal justice system?; and, (2) What predicts re-entry into the criminal justice system? For example, developmental criminal trajectory work is a robust area of youth justice research aimed at distinguishing typologies of youth with the primary goal of identifying youth at-risk for committing criminal offences, and/or those at risk for re-offending (e.g., Day \& Wiesner, 2019; Domburgh, Loeber, Bezemer, Stallings, \& Stouthamer-Loever, 2009; Domburgh, Vermeiren, Blokland, \& Doreleijers, 2009; Corrado, McCuish, Hart, \& DeLisi, 2015; McCuish, Lussier, \& Corrado, 2016). In recent years, there has also been a proliferation of youth risk assessment research. Ten years ago, Olver, Stockdale, and Wormith (2009) identified 49 unique studies for their meta-analysis on three youth-specific adaptations of risk assessment tools. Since that time, a substantial body of research regarding the development and validation of youth-specific risk assessment tools has continued to grow (e.g., Christiansen \& Vincet, 2013; Clarke, Peterson-Badali, \& Skilling, 2017; Jones, Brown, Robinson, \& Frey, 2015, 2016; Lennox, O’Malley, Bell, Shaw, \& Dolan, 2015; Pusch \& Holtfreter, 2018; Royer-Gagnier, Skilling, Brown, Moore, \& Rawana, 2016; Viljoen, Mordell, \& Beneteau, 2012; Vincent, Chapman, \& Cook, 2011). Research examining the role of responsivity factors in relation to risk reduction (i.e., rehabilitation), including studies related to youth justice service delivery models, has also emerged as an important line of study in the field (Haqanee, Peterson-Badali, \& Skilling, 
2015; Holloway, Cruise, Morin, Kaufman, \& Steele, 2018; Hubbard, 2007; McCormick, Peterson-Badali, \& Skilling, 2017; Peterson-Badali et al., 2015; Taxman, 2014; Thanner \& Taxman, 2003).

The youth justice research stemming from the criminogenic risk framework has undoubtedly advanced evidence-based correctional practice within the youth justice system; however, it has also contributed to the narrowing of the youth criminal justice system's focus toward rehabilitation outcomes, specifically recidivism. Having a youth criminal justice system narrowly focused on recidivism outcomes is problematic because it restricts what are considered the legitimate activities of the system itself, and by extension what is considered legitimate youth justice research. This is not to say the youth criminal justice system should abandon its responsibility toward the reduction of recidivism. Rather, the youth criminal justice system needs to expand its scope of responsibility toward justice-involved youth and address youth needs related to their psychosocial development regardless of whether they contribute toward the ultimate desired outcome of reduced recidivism.

Policymakers in Canada, and internationally, have acknowledged the importance of expanding the state's responsibility towards justice-involved youth. For example, in 2009 the British Columbia Representative for Children and Youth and the British Columbia Provincial Health Officer submitted a joint special report to the Legislative Assembly advocating for a youth criminal justice system focused "on the well-being and safety of children... and the state's responsibility to ensure their development continues given their immaturity" (p. 55). In a similar report entitled More Care Less Court: Keeping Youth out of the Criminal Justice System by the Office of the Child and Youth 
Advocate in New Brunswick (2015), the authors state "to address youth crime effectively...it takes a system that is built to be responsive to the developmental needs of youth...the system needs to address their therapeutic, social, educational, and vocational needs.” (p. 6). In Ontario, the Ministry of Children and Youth Services recently introduced a new Youth Justice Outcomes Framework (2015) with an explicit mandate to "move beyond recidivism as the key measure of impact." (p. 19; Elliot, 2015). An international example comes from the United Kingdom's Ministry of Justice review of the youth criminal justice system in England and Wales. A core recommendation of this report was to build a "system that moves away from justice with some welfare, to a welfare system with justice" (p. 49; Taylor, 2016).

The state's obligation to address the needs of justice-involved youth whether they fall within the scope of reducing reoffending is especially important given the potential for youth to be harmed by the criminal justice system process itself. The youth criminal justice system is an inherently adversarial system that can disrupt the social bonds and psychosocial development of justice-involved youth (Cauffman \& Steinberg, 2012; Dmitrieva, Monahan, Cauffman, \& Steinberg, 2012; Grisso, 2008; Loeber \& Farrignton, 2012; National Research Council, 2013). Consequences of this can include the onset and exacerbation of mental health issues, interference with the completion of schooling, and interference with entry into the workforce (Hirschfield, 2009; Penner, Roesch, \& Viljoen, 2011; Steinberg, 2009). If the state is going to impose such a potentially harmful process onto its youth, it must be required to mitigate and address the issues it contributes to creating. As stated, this imperative exists even if the psychosocial issues are directly related to the youth's formal criminal rehabilitation or not. 
The state has a further obligation to address the psychosocial needs of justiceinvolved youth because these needs are often the direct result of the failure of the state's responsibilities in other systems (Taylor, 2016). A stark example is the consistent failure of the child welfare system, which has led to the over-representation of youth from state custody in the youth criminal justice system in Canada, and internationally (Australian Institute of Health and Welfare, 2017; Bala, Finlay, De Filippis, \& Hunter, 2015; Corrado, Freedman, \& Blatier, 2011; Finlay, 2003; Kovarikova, 2017; Malvaso, Delfabbro, Day, \& Nobes, 2019; McFarlane, 2010; Scully \& Finlay, 2015). Failures within the education system, the public health system, and the mental health system, have also been demonstrated to lead to increased criminal justice system involvement for already vulnerable and marginalized youth (Bird, Amaechi, Bey, \& Taliaferro, 2016; Council on School Health, 2013; Foster, Qaseem, \& Connor, 2004; Kutcher \& McDougall, 2009; Heitzeg, 2016; Hirschfield, 2018; Monterastelli, 2017; Rocque \& Snellings, 2018; Salole \& Abdulle, 2015; Wald \& Losen, 2003).

It is incumbent upon youth justice researchers to contribute to the evidence needed to advance a youth criminal justice system that prioritizes the broad needs of justice-involved youth. This must include research elucidating the effects of the criminal justice system process. Doing so will help ensure the process through which justiceinvolved youth are required to navigate is as least destructive to their psychosocial development as possible. This in turn has the potential to ultimately reduce the long-term cost of justice-involved youth on other systems including the social service system, the mental health system, as well as the adult criminal justice system. 


\section{Dissertation Summary}

Among the most pressing psychosocial needs for youth in the youth criminal

justice system are mental health issues (International Society of Psychiatric-Mental Health Nurses, 2008). Policymakers and academics in Canada, and internationally, are increasingly faced with the reality that the youth criminal justice system is becoming a service for youth with mental health needs (Office of Juvenile Justice and Delinquency, 2000; Underwood \& Washington, 2016). Part of the reasoning for why mental health needs are considered a pressing priority issue is because the youth criminal justice system is not inherently designed or structured to address many of these needs. The resulting consequence of this is an ever-growing gap between the degree of mental health support required by justice-involved youth, and the degree of service available to address these needs. This is because there are substantial rates of mental health needs among this population of youth that are significantly higher compared to youth in the community (Canadian Institute for Health Information, 2008), and the youth criminal justice system is generally poorly equipped to deal with these issues. For example, in a report by the Federal Advisory Committee on Juvenile Justice (2011) in the United States, it was concluded that personnel working within the youth criminal justice system were severely hindered their ability to provide adequate mental health services because of a general lack of research, insufficient policy development, inadequate models of care, and ineffective experience and training of staff. Similar issues are faced in Canada, where only a fraction of justice-involved youth are ever screened and/or assessed for mental health issues. Formal legislative options made available through the YCJA (2002), including having a Youth Mental Health Worker, receiving a comprehensive mental health assessment, or 
receiving a specialized sentence which prioritizes mental health treatment (e.g., mandated enrollment in an Intensive Support and Supervision Program), are rarely applied (Peterson-Badali et al., 2015). As stated, this rarity stands in sharp contrast to the high rate of mental health problems in this population.

In order for the youth criminal justice system to adequately and appropriately address the mental health needs of justice-involved youth, regardless if they are related to recidivism, youth must be assessed. While there are valid and just arguments for assessing mental health needs among justice-involved youth outside of the Risk-NeedResponsivity (RNR) model (Andrews, Bonta, \& Wormith, 2006), it is important to acknowledge that some mental health issues can be criminogenic needs. Although broad mental health diagnoses are not generally empirically established as criminogenic needs, at a more granular level many facets of commonly diagnosed disorders among justiceinvolved youth are (McCormick et al., 2017). A salient example is impulsivity, which is a core diagnostic criteria for Attention-Deficit-Hyperactivity Disorder, and is explicitly identified as a criminogenic need in the RNR framework (Peterson-Badali et al., 2915). As such, youth forensic assessment is not only important from a youth welfare perspective, but also has relevance to the core criminal rehabilitation activities of the youth criminal (i.e., reduction of recidivism).

Studying the decision-making process of clinicians conducting forensic assessments will help inform our understanding of how treatment and service recommendations for justice-involved youth are determined. In the current dissertation, Study 1 examines how clinician-derived diagnoses and recommendations are arrived at when youth provide information about their mental health that is either consistent, or 
inconsistent, with information provided by their caregivers. Study 1 is couched in the developmental psychopathology literature which considers multiple informant assessments of youth as the gold standard of practice (De Los Reyes, 2013). However, few studies have examined multi-informant assessments in a forensic context, and even fewer have included clinician-derived outcomes, such as diagnoses. Given this, Study 1 advances the broader informant discrepancy literature but also the forensic assessment literature.

Understanding the mental health needs of justice-involved youth during the criminal justice system process is another important component of advancing youth criminal justice system responses designed to mitigate any potential harm to the psychosocial development of justice-involved youth. Study 2 examines how youth perceived changes in their mental health, self-esteem, and perceived social support, from before they formally entered the youth criminal justice system to after their adjudication. Specifically, youth were asked to provide retrospective pre-charge reports across a range of psychosocial variables, while simultaneously reporting their current state on the same variables. This study is couched in the forensic mental health literature and is heavily informed by previous work examining the effect of custodial placements on justiceinvolved youth. Study 2 contributes to broader forensic mental health research by asking youth about changes to their psychosocial functioning that they specifically attributed to their experience during the adjudication period of youth criminal justice system processing. The implications of the findings of Study 2 to better the criminal justice system process for youth are discussed. 
Another possible effect associated with involvement in the youth criminal justice system that can disrupt the psychosocial development of justice-involved youth is the experience of stigma. The stigma related to being involved in the criminal justice system for youth and adults is referred to as offending-based stigma. Study 3 explores if, and how, justice-involved youth experience offending-based stigma. Because a very limited body of offender-based stigma research exists, particularly in psychology, Study 3 is couched within the broader stigma literature and draws heavily from the fields of HIV/AIDS, mental health, and sexual minority stigma. Study 3 is unique in relation to Studies 1 and 2 in that it takes an explicit interdisciplinary and intersectional approach. As such, it not only contributes to the area of offending-based stigma specifically, but also contributes to the broader stigma literature by demonstrating the intersectional nature of stigma. 


\section{Chapter 2: The Effect of Youth-Caregiver Discrepancies in Forensic Assessment \\ Clinician Decision-Making}

Forensic assessments play a critical role in the adjudication, sentencing, and management of youth at the intersection of the criminal justice and mental health systems. Within the youth justice system, forensic assessments have a dual role in that they are used to make determinations about a youth's risk of reoffending, in addition to identifying underlying psychopathology and/or post-adjudication treatment and rehabilitation needs of the youth. Previous research has demonstrated clinical reports significantly influence the disposition outcomes of justice-involved youth, with some studies reporting strong correlations between clinician recommendations and judge's final decisions (e.g., Campbell \& Schmidt, 2000, Hecker \& Steinberg, 2002; Cauffman, Piquero, Kimonis, Steinberg, Chassin, \& Fagan, 2007; Schwalbe, Hatcher, \& Maschi, 2009). It is therefore important to understand which variables contribute to clinician decision-making, as these decisions have implications for how the youth criminal justice system responds to the delinquency-related - but also the psychosocial - needs of justice-involved youth.

\section{Informant Discrepancies}

As in other child/youth assessment contexts, multi-informant assessments are considered the gold standard in youth forensic assessment (Grisso, 2008; Renk, 2005; Salekin, 2015). Assessments that rely on information from multiple informants, including the youth themselves, parents or caregivers, and teachers, are considered best practice because each informant offers unique insight into the behavioural and/or emotional problems of the youth (Hunsley \& Mash, 2007). Prior theoretical models of child and 
youth behaviour suggest that multi-informant assessments are necessary because youth systematically vary in where they express certain behaviours and/or symptoms, and informants systematically vary in where they observe certain behaviours and/or symptoms (Kraemer, Measelle, Ablow, Essex, Boyce, \& Kupfer, 2003). Several empirical studies support this model by demonstrating contextual variation in the manifestation of youth mental health issues including conduct problems, attention and hyperactivity, and conduct problems (e.g., Dirks, De Los Reyes, Briggs-Gowan, Cella, \& Wakschlag, 2012; Petitclerc et al., 2015).

Discrepancies between multiple informants are among the most robust findings in developmental psychology (De Los Reyes \& Kazdin, 2005). In their influential metaanalysis of 119 studies that examined informant discrepancies, Achenbach, McConaughy, and Howell (1987) reported a mean correlation of .22 between children and youth, and other informants. The mean correlation between informants was significantly higher for children 6 to 11 years old compared to older youth, and was significantly higher for "undercontrolled" problems (i.e., externalizing behaviour) compared to "overcontrolled" problems (i.e. internalizing symptoms; Achenbach, et al., 1987). Decades of research since the publication of this meta-analysis have replicated and supported these findings, and have consistently demonstrated low to moderate agreement between youth and parent reports across a wide range of psychological constructs and assessment contexts (Collishaw, Goodman, Ford, Rabe-Hesketh, \& Pickles, 2009; Cleridou, Patalay, \& Martin, 2017; De Los Reyes, Youngstrom, Pabón, Youngstrom, Feeny, \& Findlings, 2011; Duhig, Renk, Epstein, \& Phares, 2000; Handwerk, Larzelere, Soper, \& Friman, 
1999; Kolko \& Kazdin, 1993; Renk \& Phares, 2004; Reynolds, MacPherson, Matusiewicz, Schreiber, and Lejuez, 2011).

Informant discrepancies observed throughout the field of developmental psychology can make it difficult to interpret research findings and identify best practice treatments (De Los Reyes \& Kazdin, 2008; De Los Reyes, Kundey, \& Wang, 2011). Additionally, one of the main practical challenges faced by clinicians is how to best integrate discrepant information from multiple informants into an assessment. Earlier work suggested the use of an "optimal informant" (Hart, Lahey, Loeber, \& Hanson, 1994; Loeber, Green, \& Lahey, 1990; Loeber, Green, Lahey, \& Stouthamer-Loeber, 1989; Verhulst \& van der Ende, 1991). For example, Smith (2007) outlined a method to rank the "best" informants based on a multi-dimensional framework including age, setting, and problem type. Other strategies include applying the "OR rule" whereby clinicians count a behaviour or symptom endorsed by any informant, or the "AND rule" whereby clinicians only count a behaviour or symptom if it is endorsed by all informants (Angold, 2002; Lahey et al., 1994; Martel, Markon, \& Smith, 2017; Valo \& Tannock, 2010).

Most empirical research regarding clinician decision making in relation to informant discrepancies indicates clinicians systematically rely on an optimal informant. For example, in a sample of 258 youth-parent dyads drawn from inpatient and outpatient treatment sites, Kramer and colleagues (2004) found that clinicians consistently relied on parent reports of impaired functioning instead of adolescent reports. Clinicians in this study also consistently rated problems reported only by adolescents as less serious compared to problems reported only by parents (Kramer et al., 2004). The evidence that 
clinicians tend to rely on an optimal informant reflects the traditional perspective that informant discrepancies are meaningless and thereby must be "resolved". This perspective necessitates the rejection of one informant in favour of a more "reliable" informant which can result in the loss of valuable information, and has the potential to undermine the entire clinical process.

Recently, the field of research related to multiple informants has evolved, incorporating the position that the way in which informants differ is in itself meaningful (De Los Reyes, 2013; De Los Reyes et al., 2015). An example of this change in perspective is the shift from using difference scores in informant discrepancy research to statistical methods that model interactions between informant reports (e.g., polynomial regression; Laird \& De Los Reyes, 2013; Laird \& Weems, 2011). Although the use of polynomial regression as an alternative to difference scores was proposed decades ago (see Edwards, 1994), only recently has there been a marked increase its application to discrepancy research. Difference scores are increasingly considered redundant as they cannot produce any meaningful information about discrepant reports beyond the effects accounted for by each individual informant (De Los Reyes, Ohannessian, \& Laird, 2016; De Los Reyes, Salas, Menzer, \& Daruwala, 2013).

The shift towards a multiple regression framework allows researchers to examine how the scores from each informant are related to each other while controlling the main effects of each individual informant. This approach opens up a more nuanced examination of informant discrepancies. Despite the shift toward conducting informant discrepancy research within such a multiple regression framework in the broader field of developmental psychopathology (e.g., Becker, Jensen-Doss, Kendall, Birmaher, \& 
Ginsburg, 2016; Becker-Haimes, Jensen-Doss, Birmaher, Kendall, \& Ginsburg, 2017;

Fefer, Ogg, \& Dedrick, 2015; Nelemans et al., 2016; Sibley, Campez, \& Reiker, 2017;

Tackett, Herzhoff, Reardon, Smack, \& Kushner, 2013; Vierhaus, Rueth, \& Lohaus, 2016), no studies to date have used this methodology to examine youth-caregiver discrepancies in a forensic context.

\section{Informant Reliability in the Forensic Context}

Multi-informant assessments are particularly important in a forensic context since contextual and individual factors may influence the reliability of the information provided by justice-involved youth and their parents. Justice-involved youth are vulnerable to response bias given the significant implications of forensic assessments as it relates to restrictions of freedom (e.g., informing custodial placement, sentence length, or probation conditions) and post-adjudication rehabilitation supports (e.g., qualifying for treatment services).

Although it is possible for justice-involved youth to exaggerate their clinical presentation, it is more commonly observed that such youth minimize externalizing/delinquent behaviours and internalizing symptoms (symptoms related to internal emotional states such as mood and anxiety; Grisso, 2004). For example, Branson and Cornell (2008) compared two self-report measures of psychopathology in a sample of incarcerated male youth, the Millon Adolescent Clinical Inventory (MACI) and the Personality Inventory for Youth (PIY), and only half (53.5\%) of the total sample passed the validity screening tests on both measures.

In a similar study, Breuk, Clauser, Stams, Slot, and Doreleijers (2007) examined the reliability of the Youth Self Report (YSR; Achenbach, 1991), which belongs to the 
Achenbach System of Empirically Based Assessment (Achenbach System) and is one the most widely used self-report tools to determine psychopathology in youth, in a sample of justice-involved youth with a severe psychiatric disorder in the Netherlands. Compared to the clinical comparison group, justice-involved youth reported significantly lower scores on the following first-order YSR problem subscales: aggressive behaviour, attention problems, depressed/withdrawn, somatic complaints, anxious/depressed, and social problems. Justice-involved youth also scored significantly lower on the second-order YSR subscale of internalizing problems compared to the clinical comparison group. The justice-involved youth and the clinical control group did not significantly differ on the first-order delinquent behaviour subscale or the second-order externalizing problem subscale; however, all reported scores on these subscales were within the standardized normal range which suggests underreporting on behalf of the justice-involved youth given the forensic context (Breuk et al., 2007).

These results are consistent with work conducted by Vreugdenhill, van den Brink, Ferdinand, Wouters, and Doreleijers (2006) who compared YSR problem subscale scores to clinical diagnoses derived from a structured clinical interview in a sample of incarcerated male youth. They found first-order externalizing YSR problem subscale scores (i.e., attention problems, delinquent behaviour, and aggressive behaviour) were significantly associated with diagnoses of attention-deficit-hyperactive-disorder (ADHD), oppositional defiant disorder (ODD), and conduct disorder (CD), collectively referred to as externalizing diagnoses. However, these effects were considered small $(r=.24$ for ADHD and $r=.41$ for ODD and CD), and there was a large discrepancy in the rate of participants who reported scores within the clinical range of these subscales and received 
an externalizing diagnosis. Specifically, $27 \%$ of the sample fell within the clinical range of the second-order YSR subscale of externalizing problems, whereas $76 \%$ of the sample was diagnosed with an externalizing disorder (Vreugdenhill et al., 2006). This discrepancy is consistent with more recent work by Imbach, Aebi, Metzke, Bessler, and Steinhausen (2013), who reported a high rate of clinician-derived conduct disorder diagnoses, despite a low rate of self-reported externalizing problems, among nonincarcerated justice-involved male youth.

Vreugdenhill and colleagues (2006) also found first-order internalizing YSR subscale scores (i.e., withdrawn behaviour, somatic complaints, anxious/depressed, and social problems) were not significantly associated with affective and anxiety disorder diagnoses. This is unsurprising considering that fewer than $10 \%$ of the sample reported scores within the clinical range on any of these subscales. It is important to note that while this finding might reflect minimization on the part of justice-involved youth, it may also reflect a lack of mental health literacy. It is possible justice-involved youth have difficulty identifying internalizing symptoms and/or difficulty discriminating between symptoms.

\section{Current Study}

The current study tested if the interaction between youth and caregiver reports of behavioural problems and internalizing symptoms, as measured by the Youth Self-Report (YSR) and Child Behavior Checklist (CBCL), are meaningfully associated with receiving a specific clinician-derived diagnosis or recommendation in a clinical forensic setting. Given the study outcomes are youth-centric, the central research hypothesis is that the relationship between youth scores and clinician-derived outcomes will vary depending on 
the corresponding caregiver scores. The interest in including, and examining, specifically the interaction effect between youth and caregivers is two-fold. As previously discussed, informant discrepancies often reflect inconsistencies in behavioural or symptom expression across contexts. Focusing on interpreting the interaction in the current study will provide evidence for whether knowledge of such contextual inconsistency (or consistency) are meaningfully associated with applying a diagnosis or recommendation. Focusing on the interaction effect will also contribute to deciphering whether clinicians tend to rely on specific informants in the presence (or absence) of discrepancies. As discussed, previous work suggests clinicians often tend to rely on an optimal informant. Including interaction effects in the current models will enable conclusions to be drawn about whether the tendency to rely on an optimal informant is conditional upon the existence of discrepancies, essentially determining whether the presence (or absence) of discrepancies is associated with clinical decision-making (De Los Reyes, 2013).

The current study builds on the existing literature regarding informant discrepancies in the youth forensic context in several ways. First, previous studies have demonstrated youth and caregiver reports are differentially related to clinician decisionmaking in samples of justice-involved youth. For example, Penney and Skilling (2012) tested whether Youth Self-Report (YSR), and the parallel caregiver version the Child Behavior Checklist (CBCL), scores predicted the presence or absence of a cliniciangenerated diagnosis in a sample of justice-involved youth/caregiver dyads. Results of their study indicate that youth-reported depressive and anxious symptoms significantly predicted the presence of a clinical diagnosis on the internalizing spectrum, whereas caregiver reports of these symptoms did not. The inverse was true for the externalizing 
problems, with results indicating caregiver-reported aggressive behaviors significantly predicted the presence of a clinical diagnosis on the externalizing spectrum, whereas youth reports of these behaviors did not (Penney \& Skilling, 2012). The current study extends our understanding of the relationship between youth and caregiver reports and clinician decision-making by investigating how youth-parent reports vary as a function of each other. This allows us to speak to the relationship between youth-caregiver discrepancies and clinician decisions.

Secondly, of the few previous studies that have examined youth-caregiver discrepancies in a forensic context all have relied on difference scores. For example, Skilling, Doiron, and Seto (2011) compared discrepancies in youth and parent reports of conduct problems between youth who had committed a sexual offence and youth who had committed a non-sexual offence using standardized difference scores. The results of this study indicate that the magnitude and direction of the youth-caregiver discrepancy differed between the two groups of youth. However, because the analyses were limited to difference scores this restricts our ability to interpret the discrepancies themselves. The current study avoids this limitation by utilizing a multiple regression framework, therefore building on previous work in this area.

Finally, the current study advances the broader youth justice research agenda of identifying and understanding factors that affect how justice-involved youth are processed through, and experience, the youth criminal justice system. The conclusions presented in forensic clinical reports have the potential to shift the rehabilitation trajectory of justice-involved youth (O'Donnell \& Lurigio, 2008). Considered in relation to the youth's right to participate (YCJA, 2002; Child, Youth and Family Services Act, 
2017), there exists an ethical imperative to understand how the participation of justiceinvolved youth in the forensic assessment process influences critical decision-making on their behalf. In this context, it is especially important to understand how information from justice-involved youth is considered in the clinical context in relation to caregiver information.

A discussed, caregivers are key informants in the assessment of adolescent psychopathology, and the youth criminal justice system strongly reinforces the participation of caregivers in the adjudication and rehabilitation processes. However, caregivers can impact how the information provided by youth is heard and weighed by the courts (Canadian Bar Association, 2019). As such, it is important to understand how caregiver reports, in the presence of youth reports, inform clinician decision-making.

\section{Method}

\section{Participants}

A total of 629 male youth, with an accompanying caregiver, participated in a court-ordered forensic assessment between 2001 and 2017 in Toronto, Canada. Of these dyads, $92.4 \%$ consented to the use of their clinical information for research purposes, resulting in a final sample of 580 dyads.

The forensic assessments were conducted by a range of clinicians from varying disciplines during the study period. Specifically, $68.6 \%$ of youth in the current sample were assessed by a psychiatrist and $31.4 \%$ were assessed by a psychologist. As part of the standard assessment battery, youth and caregivers completed the Youth Self-Report (YSR) and the Child Behavior Checklist (CBCL), which are two parallel measures from the Achenbach System (Achenbach, 1991; Achenbach \& Rescorla, 2001). In the current 
analysis, a dyad was defined as any youth-caregiver pair where at least one YSR/CBCL internalizing or externalizing subscale was completed by both informants. It was not necessary for youth-caregiver pairs to have a matching score on all internalizing or externalizing subscales. This approach was chosen to maximize the potential sample size for each outcome regression model conducted.

In addition to completing a battery of self-reported measures, youth participated in a semi-structured clinical interview. Caregivers also participated in an interview to provide collateral information and completed a parallel set of self-report measures. Additional relevant information was also collected from other collateral sources (e.g., probation officers, previous service providers). Based on the collected information, the psychiatrists and psychologists would make the final clinical diagnoses and supervise all report recommendations.

Sample descriptives. Table 1 presents demographic information regarding the full sample of youth included in the paired youth-caregiver dyads. Overall, the majority of youth were non-White and were referred for an assessment in response to a violent non-sexual offence. Most youth had no previous contact with the mental health system, with fewer than $5 \%$ of the full sample previously undergoing an early childhood assessment (defined as any assessment conducted prior to the age of 12), previously receiving a mental health diagnosis, or previously accessing mental health services.

Biological mothers were most often the caregiver who participated in the assessment $(80.1 \%)$, followed by biological fathers (12.9\%), sociolegal parents (i.e., adoptive, step-, and foster parents; 5.3\%), and grandparents (2.1\%). Almost all caregivers completed high school $(49.8 \%)$ or a post-secondary degree $(44.7 \%)$, with the remaining 
caregivers finishing Grade 8 (4.8\%). Annual household income was collected as a categorical variable, with $40.0 \%$ of caregivers reporting less than $\$ 30,000$ per year (considered the poverty line in Canada for a household of one caregiver and one child; Statistics Canada, 2017), 32.6\% of caregivers reporting between $\$ 30,000$ and $\$ 60,000$ per year, and $27.1 \%$ of caregivers reporting greater than $\$ 60,000$ per year. 
Table 1.

Descriptive information for full sample of youth included in paired dyads

\begin{tabular}{|c|c|c|c|c|}
\hline & \multicolumn{4}{|c|}{ Recommendations } \\
\hline & $\begin{array}{c}\text { Full } \\
N=580\end{array}$ & $\begin{array}{c}\text { Non-coded } \\
n=422\end{array}$ & $\begin{array}{l}\text { Coded } \\
n=422\end{array}$ & \\
\hline & $M(S D) / \%$ & $M(S D) / \%$ & $M(S D) / \%$ & $t$-value $/ \chi^{2}$ \\
\hline Age & $16.0(1.5)$ & $16.1(1.5)$ & $15.7(1.6)$ & $2.60^{*}$ \\
\hline \multicolumn{5}{|l|}{ Ethnicity } \\
\hline White & 32.0 & 34.8 & 24.7 & \multirow{3}{*}{$7.98^{*}$} \\
\hline Non-White ${ }^{\mathrm{a}}$ & 66.1 & 62.8 & 74.7 & \\
\hline Indigenous & 1.9 & 2.4 & 0.6 & \\
\hline \multicolumn{5}{|l|}{ Index offence } \\
\hline Non-violent & 20.3 & 21.6 & 25.3 & \multirow{3}{*}{3.89} \\
\hline Sexual offence & 20.2 & 19.4 & 24.7 & \\
\hline Violent offence & 47.2 & 59.0 & 50.0 & \\
\hline Previous criminal history ${ }^{b}$ & 15.3 & 12.8 & 21.5 & $7.03^{* *}$ \\
\hline Previous diagnosis & 20.6 & 22.6 & 16.4 & 7.31 \\
\hline \multicolumn{5}{|l|}{ Current diagnosis } \\
\hline Anxiety & 3.6 & 13.8 & 4.8 & $5.72^{*}$ \\
\hline Depression & 6.4 & 21.6 & 10.2 & $5.97^{*}$ \\
\hline Conduct disorder & 26.7 & 44.3 & 44.9 & 0.01 \\
\hline $\mathrm{ODD}^{\mathrm{c}}$ & 4.8 & 16.5 & 8.0 & $4.01^{*}$ \\
\hline $\mathrm{ADHD}^{\mathrm{d}}$ & 20.2 & 48.2 & 37.4 & 3.25 \\
\hline Any internalizing & 8.1 & 27.4 & 12.5 & $8.52^{* *}$ \\
\hline Any externalizing & 38.6 & 78.7 & 64.6 & $7.58^{* *}$ \\
\hline \multicolumn{5}{|l|}{ Clinical recommendation } \\
\hline Mental health counselling & 8.1 & --- & --- & --- \\
\hline Psychiatric medication & 7.8 & --- & --- & --- \\
\hline Anger management & 9.8 & --- & --- & --- \\
\hline Custodial placement & 4.8 & --- & --- & --- \\
\hline Educational supports & 21.7 & --- & --- & --- \\
\hline
\end{tabular}

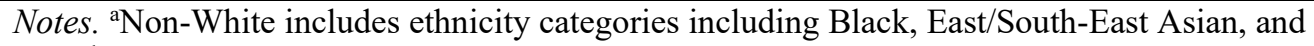
Arab. ${ }^{b}$ Previous criminal history was measured using item 1 of the Level of Youth Service/Case Management risk assessment tool. ${ }^{\mathrm{C}} \mathrm{ODD}$ refers to oppositional defiant disorder. ${ }^{\mathrm{d}} \mathrm{ADHD}$ refers to attentiondeficit-hyperactive-disorder. 


\section{Measures}

Youth Self-Report and Child Behavior Checklist (YSR and CBCL). The YSR and CBCL belong to the Achenbach System (Achenbach and Rescorla, 2001) and are two of the most widely used tools to screen for emotional, behavioural, and social problems among youth (Appendix A and B). Previous research supports the use of the Achenbach System suite in forensic youth assessment (Hoge \& Andrews, 2010; Semel, 2017).

Descriptive statistics for the YSR and CBCL are presented in Table 2. The YSR and CBCL each include 112 items (89 of which are identical between the two measures) that are rated on a 3-point scale ranging from not true, sometimes true, or very true. Higher scores reflect more problematic behavior. The YSR and CBCL provide eight firstorder subscale scores originally identified using factor analysis (Achenbach, 1991). However, because the current study is concerned with dyadic comparisons only the six first-order subscales derived from identical YSR/CBCL items are included in the analyses. This approach enables optimal comparisons between the dyads. The six firstorder subscales included are: Anxious/Depressed (e.g., I am afraid I might think or do something bad), Withdrawn/Depressed (e.g., There is very little I enjoy), Somatic Complaints (e.g., I have nightmares), Attention Problems (e.g., I daydream a lot), RuleBreaking Behavior (e.g., I break rules at home, school, or elsewhere), and Aggressive Behavior (e.g., I am mean to others). The YSR/CBCL first-order subscale scores can also be combined to provide two second-order subscale scores, Internalizing (combined scores from the Anxious/Depressed, Withdrawn/Depressed, and Somatic Complaints subscales) and Externalizing (combined scores from the Rule-Breaking Behavior and Aggressive Behavior). 
Table 2.

Descriptive statistics of $Y S R / C B C L$ scales

\begin{tabular}{|c|c|c|c|c|c|}
\hline & $n$ & $M(S D)$ & Range & $\alpha$ & $r$ \\
\hline \multicolumn{6}{|l|}{ First-order YSR/CBCL scales } \\
\hline YSR anxious/depressed & \multirow{2}{*}{493} & $5.05(4.1)$ & & .80 & \multirow{2}{*}{$.33^{* *}$} \\
\hline CBCL anxious/depressed & & $4.71(4.5)$ & $0-21$ & .83 & \\
\hline YSR withdrawn/depressed & \multirow[b]{2}{*}{506} & $4.76(3.1)$ & $0-15$ & .73 & \multirow{2}{*}{$.32^{* *}$} \\
\hline CBCL withdrawn/depressed & & $4.10(3.5)$ & $0-15$ & .80 & \\
\hline YSR somatic complaints & \multirow{2}{*}{507} & $3.12(3.1)$ & $0-14$ & .77 & \multirow{2}{*}{$.30^{* *}$} \\
\hline CBCL somatic complaints & & $2.19(2.8)$ & $0-18$ & .78 & \\
\hline YSR attention problems & \multirow{2}{*}{495} & $6.68(3.9)$ & $0-18$ & .79 & \multirow{2}{*}{$.41^{* *}$} \\
\hline CBCL attention problems & & $7.05(4.6)$ & $0-18$ & .85 & \\
\hline YSR rule-breaking behavior & \multirow{2}{*}{425} & $8.10(5.3)$ & $0-25$ & .84 & \multirow{2}{*}{$.55^{* *}$} \\
\hline CBCL rule-breaking behavior & & $8.52(6.5)$ & $0-26$ & .89 & \\
\hline YSR aggressive behavior & \multirow{2}{*}{464} & $8.89(6.1)$ & $0-30$ & .87 & \multirow{2}{*}{$.45^{* *}$} \\
\hline CBCL aggressive behavior & & $9.51(8.3)$ & $0-34$ & .93 & \\
\hline \multicolumn{6}{|c|}{ Second-order YSR/CBCL scales } \\
\hline YSR internalizing & \multirow{2}{*}{406} & $12.59(8.9)$ & $0-43$ & .89 & \multirow{2}{*}{$.38^{* *}$} \\
\hline CBCL internalizing & & $10.65(9.2)$ & $0-43$ & .90 & \\
\hline YSR externalizing & \multirow{2}{*}{363} & $16.94(10.6)$ & $0-53$ & .91 & \multirow{2}{*}{$.50^{* *}$} \\
\hline CBCL externalizing & & $18.23(14.4)$ & $0-79$ & .95 & \\
\hline
\end{tabular}

Notes. ${ }^{*} p<.05,{ }^{* *} p<.01,{ }^{* * *} p<.001$ 
Clinician-derived outcomes. Clinician-derived outcomes were coded from the youth's final forensic assessment report as either present or absent. These are divided into diagnoses and recommendations. Due to the nature of file coding, the sample size for each outcome variable varies. Tables 2 and 3 present the sample size and percentages for each clinician-derived outcome included in each analysis.

Diagnoses. Psychiatric diagnoses were made following the nosology of the Diagnostic and Statistical Manual of Mental Disorders $\left(4^{\text {th }}\right.$ or $5^{\text {th }}$ ed.; American Psychiatric Association, 2013). Only internalizing and externalizing spectrum diagnoses that align with symptoms and behavioural problems and internalizing symptoms measured by the YSR/CBCL were included the analyses. These include conduct disorder (CD), oppositional defiant disorder (ODD), attention deficit hyperactive disorder (ADHD), anxiety, and depression. CD, ODD, and ADHD diagnoses were combined into an "any externalizing diagnosis" category, and anxiety and depression diagnoses were combined into an "any internalizing diagnosis" category.

Any diagnoses related to a psychotic disorder (e.g., schizophrenia), a developmental disorder (e.g., autism spectrum disorder), a learning disability (e.g., mild intellectual disability), or trauma (e.g., post-traumatic stress disorder), were excluded because the diagnostic criteria for these disorders do not map on to the constructs measured by the YSR/CBCL measures. Many of these disorders are also rare. For example, only approximately one percent of youth in the current sample of dyads received a diagnosis for a psychotic disorder (1.3\%), post-traumatic stress disorder $(0.4 \%)$, or autism spectrum disorder $(0.9 \%)$. 
Recommendations. Clinical recommendations were coded directly from the youth's final clinical report. Following a standardized coding form, a total of 15 possible recommendations were coded as either present or absent. Given the forensic context, the 15 possible recommendations were chosen because aligned closely with empirically established criminogenic needs including: education, employment, family, substance use, peer relations, personality, anger management, and antisocial attitudes. Many of the 15 originally coded recommendations, but not all, were informed by the results of the internalizing and externalizing factors measured by the YSR/CBCL. As such, only the recommendations that mapped on to the constructs measured by YSR/CBCL were used in the analyses. For example, education related recommendations were included because the YSR/CBCL measures attention problems, whereas substance use recommendations were not included because the YSR/CBCL does not measure substance use issues.

Overall, five recommendations were included in the current analyses: mental health counselling (not otherwise specified), psychiatric medication, anger management, open or secure custody placement, and educational supports (e.g., recommendation to be place in an alternative classroom). It is also important to note only a subset of the youth included in the full sample of dyads had their final clinical reports coded for recommendations because of time and research personnel restrictions.

At the time the current analysis was conducted, clinical recommendations were coded for $27.2 \%(n=158)$ of the full sample of youth included in the paired dyads. Table 1 presents descriptive information for both the non-coded and coded recommendation sub-samples of youth. 
The sub-sample of youth whose recommendations were coded differed from the rest of the sample in two key ways. First, approximately $2.0 \%$ of the full sample were Indigenous $(n=11)$, whereas there was only one Indigenous case files coded for recommendations $(0.6 \%$ of coded cases files). Second, a significantly greater proportion of youth whose recommendations were coded and had a previous criminal history compared to the full sample $(20.3 \%$ to $29.1 \%)$.

\section{Data Analysis Plan}

Raw Youth Self-Report (YSR) and Child Behavior Checklist (CBCL) scores for each subscale were used as the independent variables in the analyses. Although YSR/CBCL T-scores are often used in research with clinical samples, Achenbach and Rescorla (2001) explicitly state raw scores should always be used when conducting any statistical analyses. This is because YSR/CBCL T-scores are truncated, meaning scores at or lower than the mean are all assigned a $\mathrm{T}$-score of 50, resulting in the loss of the bottom part of the scoring distribution. Using raw scores therefore offers superior validity and interpretability of statistical results as such results are based on the full range of variability. All raw subscale scores were mean centered to aid with the interpretation of regression coefficients and to limit multicollinearity (Aiken \& West, 1991; Cohen, Cohen, West, \& Aiken, 2003).

To test the central hypothesis, we conducted a series of multiple logistic regression analyses with interaction terms (i.e., polynomial regression). As discussed, this approach is ideal to understand how youth and caregiver scores are related because the interaction term can test multiple patterns of discrepancies that could not otherwise be captured. Specifically, the interaction term allows one to examine whether high (or low) 
scores from youth are more or less strongly associated with each clinician-derived outcome when scores from caregivers are also high (or low).

The following terms were included in the regression models: youth score, caregiver score, youth*aregiver, (youth score $)^{2}$, $(\text { caregiver score) })^{2}$. The higher order terms were included to strictly control for the possibility of a curvilinear relationship between the predictors and outcomes, and therefore will not be interpreted. The inclusion of higher-order terms is recommended as it has the advantage of reducing the likelihood of an underestimated model (Edwards, 1994). A possible disadvantage of this approach is the interaction term becomes conditional upon the higher order terms, meaning the interaction coefficient changes depending on the inclusion or exclusion of the higherorder terms. To ensure the interaction coefficients in the presented results are not conditional, all regression models were re-run using the original raw youth and caregiver scores, centred around zero. The interaction coefficients for all regression models did not change, indicating the inclusion of the higher order terms did not create conditional effects. On balance, the decision to retain the high order terms in the final models was made.

The interaction term is of most relevance to the current research question as it directly tests the fundamental discrepancy hypothesis that associations between an outcome and reports from one informant vary as a function of the other informant's report. The interaction term is essentially testing whether the association between the youth scores and the outcome is moderated by the caregiver scores. The interaction term is equally testing whether the association between the caregiver scores and the outcome is moderated by the youth scores. 
The only way to fully interpret an interaction effect is to examine the simple slope patterns. As such, post-hoc probing of significant interaction terms was conducted by plotting the simple effect coefficients at low and high levels of youth- and caregiverreported problems.

Of secondary interest are the conditional main effects of the youth and caregiver scores. In the context of the regression models, the association between the youth scores and the outcomes is interpreted at mean levels of caregiver scores. Similarly, the association between the caregiver scores and the outcomes is interpreted at mean levels of youth scores. Although these results cannot speak to the direct main effect of either the youth or caregiver scores on the outcomes, they are meaningful in the context of multiple informant research since multi-informant assessments necessitate the consideration of youth and caregiver reports together.

\section{Results}

To limit Type I error inflation, not all possible regression models were tested. Only first-order and second-order subscales that theoretically align with specific clinician-derived diagnoses and recommendations were included as predictors in the regression models. The beta coefficients, odds ratio (OR), and 95\% confidence interval for the ORs are reported for the conditional main effect of the youth and caregiver scores. ORs above 1 are interpreted as an increase in odds, while ORs below 1 are interpreted as a decrease in odds. Only the beta coefficients are reported for the interaction term, as interpreting ORs for an interaction does not provide meaningful information. $R^{2}$ for each model is also reported. 


\section{Clinician-Derived Diagnoses}

Regression results for all clinician-derived diagnosis outcome models are reported in Table 2.

Externalizing spectrum. A significant interaction term was found when modelling the effect of youth and caregiver reports of rule-breaking behaviour on receiving a conduct disorder $(\mathrm{CD})$ diagnosis. The simple slopes (Figure 1) indicate a discrepancy in the role of caregiver reports of rule-breaking behavior when clinicians make a CD diagnosis. Specifically, when youth report low levels of rule-breaking behavior, the likelihood of receiving a CD diagnosis is greater when caregiver reports are high compared to when caregiver reports are low. In contrast, when youth report high levels of rule-breaking behaviour, the likelihood of receiving a CD diagnosis remains the same regardless of whether caregivers report high or low levels of rule-breaking behaviour.

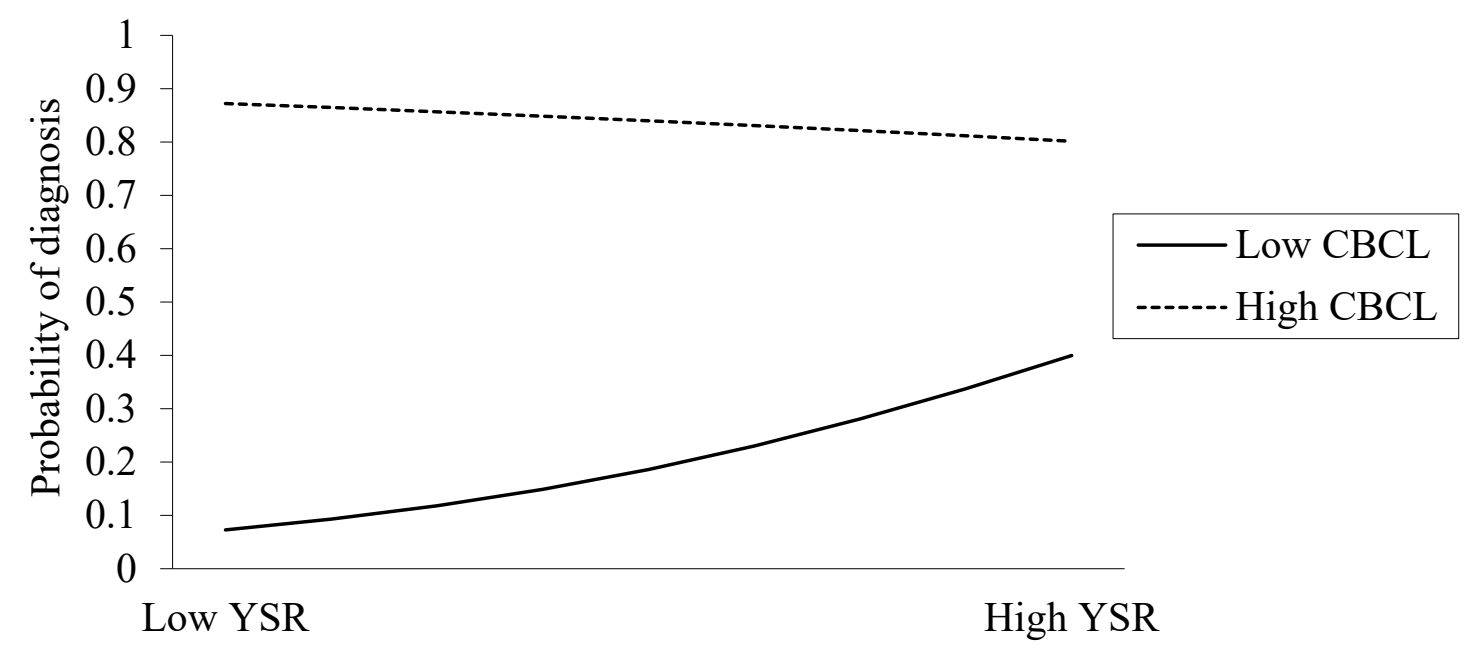

Figure 1. Probability of receiving a conduct disorder diagnosis at low and high levels of youth and caregiver reports of rule-breaking behaviours. 
No significant interaction was found when modelling the effect of youth and caregiver reports of aggressive behaviour on receiving a CD diagnosis. When examining the conditional main effects, both the youth and caregiver reports of aggressive behavior were significant predictors. In both instances, the odds of receiving a CD diagnosis were increased as youth and caregiver reports of aggressive behavior increased. However, for every unit increase on the CBCL aggressive behaviour subscale there is a $13 \%$ increase in the odds of receiving a $\mathrm{CD}$ diagnosis. This finding is in contrast to a $6 \%$ increase in odds of receiving a CD diagnosis for every unit increase on the YSR aggressive behaviour subscale. This finding suggests parent reports are more heavily weighted by clinicians when making $\mathrm{CD}$ diagnoses.

No significant interaction terms were found when modelling the effect of youth and caregiver reports of rule-breaking behavior, or aggressive behaviour, on receiving an oppositional defiant disorder (ODD) diagnosis. When examining the conditional main effects, the only significant predictor of receiving an ODD diagnosis is caregiver reports of rule-breaking behaviour. This finding indicates that at mean levels of youth reported rule-breaking behaviour, the odds of receiving an ODD diagnosis are increased as caregiver reports increase. Whereas, at mean levels of caregiver reported rule-breaking behaviour, the odds of receiving an ODD diagnosis are unchanged regardless of what youth report.

There was no significant interaction term when modelling the effect of youth and caregiver reports of attention problems on receiving an ADHD diagnosis. When examining the conditional main effects, only the caregiver scores were a significant 
predictor. This finding indicates that at mean levels of youth reported attention problems, the odds of receiving an ADHD diagnosis are increased as caregiver scores increase.

No significant interaction term was found when modelling the effect of the second-order externalizing scale on receiving any externalizing diagnosis. When examining the conditional main effects, the caregiver scores are a significant predictor of receiving an externalizing diagnosis, whereas youth scores are not. This finding indicates that at mean levels of youth reported externalizing problems, the odds of receiving an externalizing diagnosis increases as caregiver scores increase. 
Table 3.

\section{Clinical diagnosis regression results}

\begin{tabular}{|c|c|c|c|c|c|c|c|c|}
\hline \multirow[b]{2}{*}{ Predictor } & \multirow[b]{2}{*}{$n$} & \multirow[b]{2}{*}{$\%$} & \multicolumn{2}{|c|}{ YSR } & \multicolumn{2}{|c|}{ CBCL } & \multicolumn{2}{|l|}{ YSR*CBCL } \\
\hline & & & $b(\mathrm{SE})$ & $\mathrm{OR}[\mathrm{C}]$ & $b(\mathrm{SE})$ & $\mathrm{OR}(\mathrm{CI})$ & $b(\mathrm{SE})$ & $R^{2}$ \\
\hline \multicolumn{9}{|l|}{ Anxiety diagnosis } \\
\hline Anxious/depressed & 188 & 6.9 & $0.37 *(.16)$ & $1.45[1.06,1.97]$ & $0.032(.10)$ & $1.03[0.85,1.26]$ & $.01(.02)$ & .17 \\
\hline \multicolumn{9}{|l|}{ Depression diagnosis } \\
\hline Withdrawn/depressed & 205 & 17.6 & $.45^{* * *}(.13)$ & $1.57[1.22,2.02]$ & $.006(.09)$ & $1.01[0.84,1.20]$ & $.004(.02)$ & .34 \\
\hline \multicolumn{9}{|c|}{ Conduct disorder diagnosis } \\
\hline Rule-breaking behavior & 247 & 44.5 & $.06(.04)$ & $1.06[0.98,1.15]$ & $.21^{* * *}(.04)$ & $1.24[1.15,1.33]$ & $-.02^{* *}(.01)$ & .38 \\
\hline Aggressive behavior & 273 & 44.7 & $.06^{*}(.03)$ & $1.06[1.00,1.12]$ & $.13^{* * *}(.03)$ & $1.13[1.08,1.19]$ & $-.004(.003)$ & .26 \\
\hline \multicolumn{9}{|c|}{ Oppositional defiant disorder diagnosis } \\
\hline Rule-breaking behavior & 165 & 9.7 & $-.18(.11)$ & $0.84[0.68,1.04]$ & $.23^{*}(.10)$ & $1.26[1.04,1.53]$ & $.05(.03)$ & .13 \\
\hline Aggressive behavior & 189 & 11.6 & $.08(.07)$ & $1.08[0.95,1.23]$ & $.10(.06)$ & $1.11[1.00,1.23]$ & $-.001(.01)$ & .13 \\
\hline \multicolumn{9}{|c|}{ Attention-deficit-hyperactivity-disorder diagnosis } \\
\hline Attention problems & 233 & 41.2 & $.05(.05)$ & $1.05[0.95,1.16]$ & $.34^{* * *}(.06)$ & $1.40[1.25,1.58]$ & $-.004(.01)$ & .35 \\
\hline \multicolumn{9}{|c|}{ Any internalizing diagnosis } \\
\hline Internalizing & 155 & 18.1 & $.11^{*}(.05)$ & $1.12[1.02,1.24]$ & $.01(.05)$ & $1.01[0.92,1.01]$ & $.004(.003)$ & .37 \\
\hline \multicolumn{9}{|c|}{ Any externalizing diagnosis } \\
\hline Externalizing & 183 & 69.9 & $.01(.03)$ & $1.01[0.97,1.07]$ & $.11^{* * *}(.02)$ & $1.11[1.07,1.16]$ & $-.004(.002)$ & .40 \\
\hline
\end{tabular}

Notes. ${ }^{*} p<.05^{* *} p<.01{ }^{* * *} p<.001$ 
Internalizing spectrum. No significant interaction terms were found when modelling the effect of youth and caregiver reports of internalizing symptoms on receiving an anxiety diagnosis, a depression diagnosis, or any internalizing diagnosis. This result is contrary to what was hypothesized, and suggests the relationship between youth reports of internalizing symptomology and internalizing spectrum diagnoses does not change depending on caregiver reports of internalizing symptomology. In other words, caregiver reports do not moderate the relationship between youth reports and outcomes when it comes to internalizing spectrum diagnoses.

When examining the conditional main effects, the youth scores were significant across all internalizing diagnosis models and the caregiver scores were not. This finding indicates that at mean levels of caregiver reports, the odds of receiving an anxiety diagnosis, a depression diagnosis, or any internalizing diagnosis increases as youth scores increase.

No significant interaction term was found when modelling the effect of the second-order internalizing scale on receiving any internalizing diagnosis. When examining the conditional main effects, the youth scores are a significant predictor of receiving an internalizing diagnosis, whereas caregiver scores are not. This indicates that at mean levels of caregiver-reported internalizing symptomology, the odds of receiving an internalizing diagnosis increases as youth scores increase.

Overall, when it comes to clinicians applying specific DSM diagnoses to youth in the current sample, there is some evidence that the relationship between youth reports and receiving a diagnosis varies depending on caregiver reports. In other words, there is some evidence that caregiver reports moderate the effect of youth reports on DSM diagnosis 
outcomes; however, this evidence is exclusive to the externalizing spectrum. The results indicate a paradoxical relationship between youth and caregiver reports. Specifically, when it comes to the internalizing spectrum, clinicians largely base their decisions on youth reports, but when it comes to the externalizing spectrum, clinicians largely rely on caregiver reports. The one exception is the first-order aggressive behavior scale, where scores from both youth and caregiver significantly contributed to the diagnosis of CD.

\section{Clinician-Derived Recommendations}

Regression results for all clinician recommendation models are reported in Table 3. As discussed, the total number of cases included in each regression model varies depending on available information, and is reported in the table. The proportion of youth within each set of cases who received a specific recommendation is also reported in the table. 
Table 4.

Clinician recommendation regression results

\begin{tabular}{|c|c|c|c|c|c|c|c|c|}
\hline \multirow[b]{2}{*}{ Predictor } & \multirow[b]{2}{*}{$n$} & \multirow[b]{2}{*}{$\%$} & \multicolumn{2}{|c|}{ YSR } & \multicolumn{2}{|c|}{ CBCL } & \multicolumn{2}{|l|}{ YSR*CBCL } \\
\hline & & & $b(\mathrm{SE})$ & OR [C] & $b(\mathrm{SE})$ & $\mathrm{OR}(\mathrm{CI})$ & $b(\mathrm{SE})$ & $R^{2}$ \\
\hline \multicolumn{9}{|c|}{ General mental health counselling } \\
\hline Anxious/Depressed & 93 & 41.9 & $.05(.07)$ & $1.05[0.91,1.22]$ & $.13(.08)$ & $1.14[0.97,1.32]$ & $.01(.01)$ & .08 \\
\hline Withdrawn/Depressed & 95 & 36.8 & $.04(.09)$ & $1.05[0.88,1.24]$ & $-.01(.09)$ & $0.99[0.83,1.19]$ & $.03(.03)$ & .03 \\
\hline Internalizing & 73 & 42.5 & $.02(.04)$ & $1.02[0.94,1.10]$ & $.04(.05)$ & $1.04[0.95,1.14]$ & $.01(.01)$ & .08 \\
\hline \multicolumn{9}{|l|}{ Psychiatric medication } \\
\hline Anxious/Depressed & 119 & 28.6 & $-.001(.08)$ & $1.00[0.86,1.16]$ & $.20^{* *}(.08)$ & $1.22[1.04,1.42]$ & $.01(.01)$ & .14 \\
\hline Withdrawn/Depressed & 124 & 29.8 & $-.13(.09)$ & $0.88[0.74,1.05]$ & $.30^{* *}(.10)$ & $1.35[1.11,1.64]$ & $.01(.03)$ & .23 \\
\hline Attention problems & 122 & 27.0 & $-.01(.08)$ & $0.99[0.85,1.16]$ & $.38^{* * *}(.11)$ & $1.46[1.19,1.80]$ & $-.03(.02)$ & .34 \\
\hline Internalizing & 95 & 25.3 & $-.08(.06)$ & $0.92[0.82,1.04]$ & $.21^{* *}(.08)$ & $1.24[1.07,1.44]$ & $.03(.01)$ & .31 \\
\hline \multicolumn{9}{|l|}{ Anger management } \\
\hline Aggressive behavior & 115 & 38.3 & $.11^{*}(.05)$ & $1.11[1.01,1.23]$ & $.06(.04)$ & $1.07[0.99,1,15]$ & $<.001(.004)$ & .16 \\
\hline \multicolumn{9}{|c|}{ Custody recommendation } \\
\hline Rule-breaking behavior & 108 & 16.7 & $-.09(.10)$ & $0.91[0.75,1.11]$ & $.32^{* *}(.11)$ & $1.4[1.11,1.70]$ & $-.03(.02)$ & .28 \\
\hline Aggressive behavior & 115 & 17.4 & $-.02(.06)$ & $0.98[0.87,1.11]$ & $.13^{*}(.06)$ & $1.14[1.02,1.27]$ & $-.01(.01)$ & .19 \\
\hline Externalizing & 89 & 18.0 & $-.03(.05)$ & $0.97[0.88,1.07]$ & $.10^{*}(.05)$ & $1.10[1.01,1.21]$ & $-.01(.004)$ & .29 \\
\hline \multicolumn{9}{|l|}{ Educational supports } \\
\hline Attention problems & 122 & 75.4 & $.10(.10)$ & $1.11[0.91,1.36]$ & $.12(.06)$ & $1.12[0.99,1.27]$ & $-.01(.03)$ & .18 \\
\hline Aggressive behavior & 115 & 75.7 & $.01(.05)$ & $1.01[0.91,1.11]$ & $.09^{*}(.04)$ & $1.09[1.01,1.18]$ & $.003(.01)$ & .10 \\
\hline
\end{tabular}

Notes. ${ }^{*} p<.05^{* *} p<.01{ }^{* * *} p<.001$ 
General mental health counselling. No significant interaction terms were found when modelling the effect of youth and caregiver reported internalizing symptoms on receiving a recommendation for mental health counselling. The first-order anxious/depressed, and withdrawn/depressed scales were tested, along with the secondorder internalizing scale. Across all models no significant predictors were found, suggesting clinicians recommended mental health counselling independent of youth and caregiver scores on any of these scales. This is reflected in the low proportion of variance explained by these models $(8 \%, 3 \%$, and $8 \%$, respectively).

Psychiatric medication. No significant interaction terms were found when modelling the effect of youth and caregiver reported internalizing symptoms, or attention problems, on receiving a recommendation for psychiatric medication. The first-order anxious/depressed, withdrawn/depressed, and attention problems scales were tested, along with the second-order internalizing scale. Across all models, the conditional main effects of caregiver scores were significant predictors, whereas the youth scores were not. This indicates that at mean levels of youth reported internalizing symptoms, and attention problems, the odds of clinicians recommending psychiatric medication increase as caregiver reports increase. The largest ORs across all study models is observed when examining caregiver scores on the attention problems scales, with an almost $50 \%$ increase in the odds of clinicians recommending psychiatric medication for every unit increase in caregiver scores.

Anger management. No significant interaction term was found when modelling the effect of youth and caregiver reported aggressive behavior on receiving a recommendation for anger management treatment. When examining the conditional main 
effects, only youth reported aggressive behavior was a significant predictor. This finding indicates that at mean levels of caregiver reported aggressive behavior, the likelihood of clinicians recommending anger management treatment increases as youth scores increase.

Custody recommendation. No significant interaction terms were found when modelling the effect of youth and caregiver reported externalizing problems on receiving a recommendation to be placed in a custody setting. The first-order rule-breaking behavior, and aggressive behavior scales were tested, along with the second-order externalizing scale. Across all models the conditional main effects of caregiver scores were significant predictors, whereas youth scores were not. This finding indicates that at mean levels of youth reported externalizing problems, the odds of clinicians recommending a custody placement increases as caregiver reports increase.

Educational supports. No significant interaction terms were found when modelling the effect of youth and caregiver reported attention problems, or aggressive behavior, on receiving a recommendation for educational supports. When examining the first-order attention problems scale, neither youth or caregiver scores were significant predictors. When examining the first-order aggressive behavior scale, the caregiver scores were a significant predictor, whereas the youth scores were not. This finding indicates that at mean levels of youth reported aggressive behavior, the odds of clinicians recommending educational supports increases as caregiver reports increase.

Overall, when it comes to clinician recommendations there is no evidence to indicate the relationship between youth reports and specific recommendations varies depending on caregiver reports. The general alignment of clinical recommendations with 
caregiver reports instead of youth reports has important implications for how youth may take-up the recommendations given to them.

\section{Discussion}

The current study makes a unique contribution to the informant discrepancy and forensic assessment literatures by examining youth-caregiver reports in a clinical forensic setting. By specifically examining the relationship between youth-caregiver reports, and clinician-derived diagnoses and recommendations, the current study also aligns with the strategic direction of informant discrepancy research put forth by Doe Los Reyes (2013).

Overall, agreement between youth and caregiver reports of externalizing behaviours and internalizing symptoms was as expected. The mean correlation between youth-caregiver informants for externalizing subscales was .47 , while the mean correlation for internalizing subscales was .32. These findings are consistent with Achenbach's (1987) original meta-analysis whereby higher correlations were observed between informants for externalizing issues. It is also consistent with work by McConaughy (1992), and more recent findings by Youngstrom, Loeber, and StouthamerLoeber (2000) who specifically examined YSR and CBCL informant agreement. Youth in the current sample also reported higher levels of internalizing symptoms compared to caregivers, and caregivers reported higher levels of externalizing problems compared to youth. This is consistent with the existing literature on differences in reporting between the internalizing and externalizing spectrums (Aebi et al., 2015; Cleridou et al., 2017; Herjanic \& Reich, 1997). This differential responding pattern is the basis for which to expect youth-parent reporting discrepancies, and that such discrepancies will influence clinician-decision making. 


\section{Clinician-Derived Diagnoses}

Contrary to what was expected, study results did not yield consistent evidence that youth-parent reporting discrepancies were associated with clinician-derived DSM diagnoses. In other words, the regression results indicate caregiver reports did not moderate the relationship between youth reports and clinician-derived diagnoses (and vice versa). The one exception was in relation to the diagnosis of conduct disorder (CD) where the interaction coefficient of youth and caregiver reports of rule-breaking behaviour was significant. However, the interaction coefficient $(b=-0.02)$, and corrssponding slope, was very small suggesting a very weak moderation effect.

The current study findings indicate that youth and caregiver reports are weighted and integrated differently depending on whether the clinician is making an externalizing or internalizing spectrum diagnosis. The pattern of results shows that when applying externalizing spectrum diagnoses, caregiver reports carry the most weight. This was especially evident in relation to ADHD, where the odds of receiving an ADHD diagnosis increased by $40 \%$ for every increase in attention problems reported by caregivers. In contrast, the odds of receiving an ADHD diagnosis only increased by $5 \%$ for every increase in attention problems reported by youth. This aligns with empirically-validated ADHD diagnosis best practice guidelines, which state parent reports should be prioritized over youth reports (Rubio-Stipec, Fitzmaurice, Murphy, \& Walker, 2003; Sibley et al., 2017).

In regards to the internalization spectrum, the pattern of results suggest that when applying an anxiety or depression diagnosis youth reports carry the most weight in the decision-making process. This is consistent with the clinical perception that youth reports 
are most useful when gauging internalizing symptoms. It is also consistent with previous research showing clinicians tend to rely on an optimal informant, particularly for internalizing symptoms (Jensen et al., 1999; Klonsky, \& Oltmanns, 2002).

The current findings regarding internalizing diagnoses are not consistent with previous work by Vreugdenhill and colleagues (2006) who did not find a significant association between YSR internalizing subscale scores and clinician-derived anxiety or affective diagnoses. It is possible this inconsistency is because the clinicians in the current study were aware of the YSR when making clinical decisions, whereas in Vreugdenhill and colleague's (2006) study the YSR was administered independent of the diagnostic interview. These inconsistent findings provide support for integrating selfreport measures into the clinical decision-making process, as the current findings suggest doing so yields diagnoses that are better aligned with youth-centered reports of their own feelings. This clinical alignment is important from a youth-centered perspective as it contributes to the validation of the youth experience.

The current study did not find support that youth and caregiver reports vary as a function of each other in relation to clinician-derived diagnoses. However, the use of a multiple regression framework to examine the association between youth and caregiver reports, and clinician-derived diagnoses in a forensic sample provides valuable information to the area of youth forensic assessment. Specifically, the current findings add to the evidence that multiple informant assessments are important in the assessment of mental health issues among justice-involved youth. When conducting a comprehensive forensic assessment, the current findings indicate youth and caregiver reports are not interchangeable. This supports the concept of an informant gradient, which states certain 
informants provide more relevant diagnostic information compared to others (Kuhn et al., 2017). This is similar to the concept of an optimal informant except the informant gradient is based on relative strength of association. In general, the informant gradient is conceptualized as youth reports are less relevant than caregiver reports, but caregiver reports are less relevant than youth and caregiver reports combined. The current findings support the fact that utilizing youth and parent informants in the diagnosis of mental health issues among youth is the best approach. However, the current findings suggest caregiver reports are more relevant than youth reports when it comes externalizing spectrum issues, whereas youth reports are more relevant than caregiver reports when it comes to internalizing spectrum issues. These findings add important nuance to the conclusions drawn by Kuhn and colleagues (2017) who suggested parents should always be prioritizes when assessing global symptomology among youth.

\section{Clinician-Derived Recommendations}

As with the clinician-derived diagnoses, study results did not yield any evidence that youth-parent reporting discrepancies were meaningfully associated with clinicianderived recommendations. In terms of general mental health counselling, youth and caregiver reports of internalizing spectrum symptoms, which theoretically map on to the need for mental health counselling, were not significant predictors for this recommendation. A possible explanation for this finding is clinicians recommended mental health counselling in relation to externalizing spectrum issues, as opposed to internalizing spectrum issues, given externalizing issues are more closely related to criminal offending and the goal of the forensic assessment recommendations is to inform rehabilitation measures. To test this alternative explanation, post hoc regression analyses 
were conducted with the first-order rule-breaking and aggressive behaviour subscales, and the second-order externalizing subscale, as predictors. Overall, the same pattern of findings was observed as with the internalizing spectrum subscales, with no significant interaction terms or significant main effects. In light of these post hoc findings, it is possible that while the YSR and CBCL scores inform clinician decision-making in relation to mental health diagnoses, they may not inform the clinician's perceived need for treatment. It is also possible, given the relative specificity of the YSR and CBCL subscales, clinicians use these scores to inform recommendations related to specialized treatment not captured in the current data, as opposed to general mental health counselling. Regardless of how YSR and CBCL scores are used to inform other treatment recommendations, based on the current findings, it is likely other sources of information inform the clinician decision-making process regarding the recommendation of general mental health counselling.

Psychiatric medication was a listed recommendation in approximately $30 \%$ of the coded reports. The current findings indicate caregiver reports of internalizing symptoms were weighted more in the clinical decision-making process to recommend psychiatric medication compared to youth reports of internalizing symptoms. As caregivers reported greater internalizing spectrum symptoms, the odds of youth receiving a recommendation for psychiatric medication increased anywhere from $22 \%$ to $46 \%$ depending on the specific subscale. Given the previous finding that youth reports were weighted more compared to caregiver reports when applying internalizing spectrum diagnoses, it would seem to follow that youth reports should also be weighted more when making 
recommendations related to psychiatric medication. However, this is not the pattern that is observed in the current findings.

The findings regarding psychiatric medication raise important considerations for the application of mental health treatment recommendations in the forensic setting. Specifically, compliance with any mental health treatment recommended in a forensic context is implicitly coerced due to the adversarial and coercive nature of the criminal justice system. Although mental health treatments are not legally mandated per se, youth may still feel coerced given the forensic context. This is because youth may not be fully aware of their right to refuse treatment in a forensic context, and the right to refuse does not preclude the judicial system from imposing consequences for non-compliance. It is important to note, these consequences may be imposed in direct response to noncompliance of treatment (e.g., not following psychiatrist's orders) or in indirect response to non-compliance (e.g., not participating in anger treatment which results in ongoing confrontations with peers). Adding to this, youth may also feel coerced if the recommendation for treatment stems primarily from information provided by their caregivers and not themselves. As such, special attention should be paid to how mental health treatment recommendations are arrived at for justice-involved youth. Knowing which informant is driving specific treatment recommendations is an essential step to ensuring patient-centered approaches are incorporated in the youth forensic context, which may ultimately reduce the potential for youth to feel coerced.

Caregiver reports of externalizing behaviours were weighted more heavily in the decision-making process regarding custody placement recommendations compared to youth reports. The odds of youth being recommended to a custody facility were increased 
by $40 \%$ for every increase in rule-breaking scores reported by caregivers, suggesting scores on the CBCL rule-breaking subscale was the driving factor for this recommendation. A possible explanation for this finding is that clinicians relied on the most severe report of rule-breaking behaviour to make a custodial recommendation, which in the current study was provided by caregivers. This possible explanation is consistent with the fact a custodial placement is the most restrictive, and arguably most serious, recommendation clinicians can make in a forensic context. As such, there needs to be compelling evidence of severe behavioural disruption to warrant this type of recommendation. Given caregivers reported much greater rule-breaking problems compared to youth, it therefore follows that this information would be prioritized when making a recommendation for a custodial placement. It is important to note, there is high overlap between the items of the YSR/CBCL rule-breaking subscale and other key pieces of information, such as previous criminal history and index offence details, which would be used to make a custodial recommendation in a youth forensic assessment context. As discussed, the composition of the sub-sample whose records were coded for recommendation generally included higher risk youth. Approximately $30 \%$ of the subsample had a previous criminal history (defined as at least 3 prior convictions), which likely contributed to more severe rule-breaking scores. This adds to the proposed explanation that clinicians relied on the most severe reporting of rule-breaking behaviour to make a custodial recommendation, as more severe scores likely were more consistent with the other facts of the case (i.e., previous criminal history). It is also important to acknowledge there is some debate within the field of youth forensic assessment as to whether it should be within the purview of clinicians to make custodial placement 
recommendations on behalf youth. The inclusion of a custodial placement as a recommendation in the current study is not meant to imply custodial recommendations should or should not be included in youth forensic assessment reports. Rather, the purpose of presenting these findings was to shed light on the decision-making process behind applying such a recommendation if, and when, it is included.

\section{Limitations and Future Directions}

Although the overall sample size of the current study was large, the number of cases included in each regression model was substantially reduced due to inconsistencies in the available information. It is therefore possible that some of the analyses were underpowered due to the limited sample size and the relatively large number of parameters included in the model. This may have reduced our overall ability to detect statistically significant effects.

Another possible limitation is the majority of caregiver reports were provided by biological mothers. Previous research has suggested mothers can over-estimate conduct problems compared to fathers, especially for male children, which may have affected the magnitude of CBCL externalizing subscale scores (Christensen, Margolin, \& Sullaway, 1992; Duhig et al., 2000; Jensen, Traylor, Xenakis, \& Davis, 1988). That being said, there remains limited research regarding the effects of caregiver gender on internalizing and externalizing reports. This is especially true for research regarding youth, as most of the existing work on caregiver gender focuses on caregiver reports of elementary schoolaged children (6-12 years old). Further, no studies to date have examined the effects of caregiver gender in a forensic context. Of particular relevance may be the intersection of caregiver gender and previous criminal history. For example, it is possible fathers with a 
previous criminal history may provide a different of pattern of caregiver reports compared to mothers without a previous criminal history due to their own previous experience with forensic assessment. Overall, future research should examine how caregiver gender effects informant agreement (or disagreement) in a forensic context.

Other factors that have been empirically demonstrated to specifically influence maternal reports of youth conduct problems and internalizing problems include motherchild conflict, maternal stress, and maternal depression (Aebi et al., 2017; De Los Reyes \& Kazdin, 2006; Ehrlich, Cassidy, \& Dykas, 2011; Fjermestad, Nilsen, Johannessen, \& Karevold, 2017; Grills \& Ollendick, 2003; Najman et al., 2001; Youngstrom et al., 2000). None of these factors were controlled for in the current study which may have affected the magnitude of the observed interaction effects.

Given maternal factors play an important role in shaping informant discrepancies related to psychopathology, future work should also examine how these factors influence clinician-derived recommendations in the context of multi-informant assessments. The current findings highlight clinician recommendations are largely driven by caregiver reports of behavioural problems and internalizing symptoms. It logically follows that if maternal factors such as stress and depression affect the reporting of behavioural problems and internalizing symptoms, then they must indirectly affect clinician recommendations. However, it would benefit the field of informant discrepancy research to empirically establish such a model. Doing so would provide clinicians additional guidance on how to best integrate caregiver reports into the clinical recommendation process. 
Identifying whether the pattern of results observed regarding clinician recommendations in the current study are specific to the forensic context should also be a priority for future work. Based on the research previously discussed regarding the tendency for caregivers to be considered the optimal informants, it is likely clinicians in non-forensic settings also give more weight to caregiver reports than youth reports when making clinical recommendations for treatment. However, this cannot be assumed given clinical best practices are increasingly moving toward greater patient-centered care and rights-based approaches to treatment (Ambresin, Bennett, Patton, Sanci, \& Sawyer, 2013). It is therefore possible clinical recommendations in non-forensic contexts are increasingly based on youth reports instead of caregiver reports.

Determining whether the tendency to rely on caregiver reports when making clinical recommendations is specific to the forensic context has important implications for the equitable treatment of justice-involved youth in the court process. This is especially true for older youth, as they have a greater right to be heard in the court process compared to younger youth, due to their relative maturity, and because they have the potential to be given more severe dispositions compared to younger youth (Canadian Bar Association, 2019; YCJA, 2002). Although research has shown age is not a significant moderator of youth-caregiver discrepancies when it comes to reporting psychopathology in forensic and non-forensic contexts (e.g., Cleridou et al., 2017; De Los Reyes et al., 2015; Penney \& Skilling, 2011), this does not necessarily mean clinicians consider age when making clinical recommendations. In a study by O'Donnell and Lurigio (2008), age did not significantly predict whether clinicians recommended a community or custodial placement. In the same study, age also did not significantly 
predict whether judges ordered a community or custodial sentence (O’Donnell \& Lurigio, 2008). While these findings suggest age may not be a moderator of decisions regarding custodial placement, it is possible age is a moderator of other rehabilitative recommendations, particularly those regarding psychosocial factors.

Future work regarding the relationship between informant discrepancies and clinical recommendations in the forensic context should also examine the degree of recommendation follow-through. As discussed, youth may be less likely to followthrough on clinical recommendations derived primarily from caregiver reports. This in turn may have a negative effect on their compliance with probation and/or sentencing orders. Alternatively, it is also possible youth may be more likely to follow-through on such clinical recommendations because they have additional support from their caregivers to do so. Specifically, if caregiver reports are used to make clinical recommendations this assumes greater caregiver "buy in" to the issue. It is possible that such increased "buy in" on the part of caregivers would increase the likelihood of overall follow-through. This may be particularly true in a forensic context, as there is some evidence to suggest that youth compliance with probation conditions is related to how legitimate their caregivers perceive these conditions (Cavanagh, \& Cauffman, 2015; Vidal \& Woolard, 2016, 2017). The relationship between youth-caregiver discrepancies and recommendation follow-through may also be mediated by the age of the youth. There are mixed findings regarding the effect of age on probation compliance (e.g., Morgan, 1993; NeMoyer et al., 2014; Schwalbe \& Maschi, 2008); however, the degree to which youth-caregiver discrepancies predict recommendation compliance among justiceinvolved youth may be mediated by youth age. 


\section{Chapter Bridge: From Post-Adjudication Forensic Assessments to Pre-Post Adjudication Changes in Mental Health}

\section{Study 1 Summary}

Study 1 contributes to the area of youth forensic assessment by identifying how clinician decision-making is differentially influenced by youth and caregiver reports of externalizing behaviours and internalizing symptoms. As discussed, forensic assessments are an important part of the criminal justice system process for many youth, and advancing research, and best practices, in this area is critically important to ensuring justice-involved youth receive appropriate mental health services.

The findings from Study 1 provide insight to the degree to which the youth voice is integrated in the forensic assessment process in relation to caregiver reports. The general conclusion of Study 1 is that youth reports are prioritized differentially based on the clinical decision being made (e.g., externalizing versus internalizing diagnosis). This, in turn, has implications for how the youth voice is considered in judicial outcomes. For example, the downstream consequence of relying on caregiver reports when making clinical recommendations, is that judicial decisions based on said recommendations are also being made based on caregiver reports.

Basing judicial decisions primarily on reports from caregivers, or other collateral sources, instead of youth is not necessarily problematic; however, it does reflect an existing tension in the youth criminal justice system between balancing the best interests of the child and youth's right to autonomy and participation in the court process - all of which are subsumed under the United Nations Convention for the Rights of the Child (UNCRC; United Nations General Assembly, 1989) that Canada is a signatory to. 
Although the YCJA (2002) preamble references the Charter of Rights of Freedoms and the UNCRC, only the legal concept of the best interests of the child is explicitly referenced throughout the Act. To date, the only UNCRC right that has gained status as a priority as a legal principle in Canada is the best interests of the child principle, meaning it has primacy over other rights when interpreting the law (Paré, 2017). For example, in A.C. v. Manitoba (2012) the Supreme Court of Canada ruled on the issue of consent in health care, and stated that youth require protection sometimes at the expense of their autonomy rights. The divorcing of the best interests of the child principle from other youth rights outlined in the UNCRC in the Canadian legal landscape has contributed to a general disconnect between the law and youth rights throughout the youth justice system. This is evidenced by several case law analyses that have consistently shown that youth's protected fundamental rights are applied inconsistently and with difficulty (Ahmed, 2013; Bala, 2004; Paré, 2012, 2017; Pearson, 2015).

The purpose of bringing forward this discussion is to highlight how the findings of Study 1 do not exist in isolation from the broader legal context that struggles to appropriately consider and balance youth rights. The evidence presented above also highlights how the YCJA (2002) restricts the conceptualization of youth welfare in the youth justice system to legal definitions rooted in the best interest of the child principle. Meaning, while the preamble of the YCJA (2002) implies the Canadian youth justice system is premised on welfare-based principles by referencing "developmental challenges and needs of young persons" (p.1), and referencing the UNCRC (United Nations General Assembly, 1989), this is not necessarily how the legislation is interpreted and applied in practice. In fact, the evidence above suggests the YCJA (2002) 
divorces itself from the promotion of welfare-based approaches to youth justice by prioritizing the BIC principle above other UNCRC principles which align with a youth welfare perspective (Pearson, 2015).

Although Study 1 was not conducted within an explicit human rights framework, and does not directly address youth rights, the findings nonetheless contribute to our understanding of how decisions are made for youth in the youth criminal justice system. Because one cannot separate how these decisions are made from the context within which they are made, Study 1 has implications for youth rights, and by extension, youth welfare approaches across in the youth criminal justice system as a whole. In this way, Study 1 ties back to the aim of this dissertation which is to advance a youth-centered and welfarebased youth criminal justice system.

\section{Study 2 Bridge}

The typical purpose of forensic assessments in the youth criminal justice system is to inform post-adjudication judicial decisions such as sentence type and severity, probation conditions, and custodial treatment (Salekin, 2015). As such, Study 1 contributes to what we know about the post-adjudication period of the youth criminal justice system.

Study 2 shifts focus from the post-adjudication period of the youth criminal justice system process to the adjudication period. Across the youth justice research landscape, it remains relatively unclear how justice-involved youth experience the adjudication process, and there is a dearth of research specifically asking youth for their perspectives. As discussed, most youth justice research emphasizes risk-based research, which by definition is oriented toward what happens to justice-involved youth after they 
leave the youth criminal justice system, and rarely utilizes data directly provided by youth themselves. Study 2 therefore fills a gap in the youth justice research landscape by examining youth reports of the effects of upstream youth criminal justice system involvement.

Prioritizing differences in offence type. The subsequent two studies emphasise comparisons between youth who have committed sexual offences and youth who have committed non-sexual offences. How youth respond to being in the criminal justice system is complex and can be influenced by a range of factors including demographic characteristics and degree of social support. Among those factors is the type of offence the youth has committed, specifically whether they have committed a sexual or nonsexual offence (Doob \& Gartner, 2013; Letourneau \& Caldwell, 2013; Meloy, Boatwright, \& Curtis, 2013; Mercado, Alvarez, \& Levenson, 2008; Sample \& Kadleck, 2008; Wright, 2015; Zimring, 2004).

Distinguishing youth based on offence type is relevant for the next study, Study 2, because youth who commit sexual offences often report greater psychological distress compared to youth who commit non-sexual offences. In a meta-analysis of 59 studies comparing male youth who committed a sexual offence to those who committed a nonsexual offence, Seto and Lalumière (2010) found that youth who committed a sexual offence were significantly more likely to experience symptoms of anxiety, social anxiety, low self-esteem, and higher levels of social isolation and withdrawal compared to youth who committed a non-sexual offence. It is not possible to determine whether the significant group differences reported are because youth who committed a sexual offence experienced greater psychopathology prior to committing their offence, and/or because 
psychopathology emerged after being identified a sexual offender in the criminal justice system. As highlighted by Seto and Lalumière (2010), it is important to identify the timing of psychopathology among youth who have committed a sexual offence as it has important implications for etiological theories of youth sexual offending that emphasize the role of affective dysregulation and emotional incompetence. Further, understanding differences in the manifestation of mental health issues among these groups of youth as they progress through the criminal justice system can help inform specialized and targeted intervention plans. 


\section{Chapter 3: Changes in Mental Health, Self-Esteem, and Social Support Among Justice-Involved Youth}

The global prevalence rate of any mental health disorder, including behavioural disorders such as conduct disorder, among justice-involved youth is approximately $90 \%$ (Abram et al., 2004; Drerup, Croysdale, \& Hoffman, 2008). Approximately 40 to $60 \%$ of justice-involved youth meet diagnostic criteria for at least one mental disorder, excluding conduct disorder, attention deficit/hyperactivity disorder (ADHD), or substance-use disorder (Public Safety Canada, 2017; Young, Moss, Sedgwick, Fridman, \& Hodgkins, 2015). There is also consistent evidence that rates of mental health problems are significantly higher among justice-involved youth compared to youth in the general population. For example, in Canada the prevalence of mental health problems among justice-involved youth is up to four times greater than the general population of youth (Canadian Institute for Health Information, 2008). Similar disproportionately high rates of mental health problems among justice-involved youth are also observed in Australia and New-Zealand (Bickel \& Campbell, 2002; Justice Health \& Forensic Mental Health, \& Juvenile Justice NSW, 2017; The Royal Australasian College of Physicians, 2011), the Netherlands (Vreugdenhil, et al., 2004), the United-Kingdom and Wales (Lader, Singleton, \& Meltzer, 2003; Lennox, Bell, O’Malley, Shaw, \& Dolan, 2013; Youth Justice Board for England and Wales, 2016), and the United-States (Merikangas et al., 2010; Development Services Group, 2017; Seiter, 2017).

\section{Mental Health and Youth Offending}

To explain the overrepresentation of youth with mental health problems in the criminal justice system, researchers have prioritized two lines of inquiry. The first line of 
inquiry has focused on identifying shared risk factors between mental health problems and criminal offending among youth that may account for the high rates of mental health problems observed. The second line of inquiry has focused on determining whether the high rates of mental health problems observed are because of a causal relationship between mental health problems and criminal offending among youth (McCormick et al., 2015).

Shared risk explanation. The shared risk explanation posits that high rates of mental health issues are observed among justice-involved youth because mental health issues and criminal offending have shared etiological risk factors. Strictly from a probability perspective, youth with certain risk factors are more likely to have mental health problems, and more likely to criminally offend. Although the exact mechanism(s) between a specific risk factor and mental health problems, and the same risk factor and criminal offending, may be similar or different, the risk factor increases the statistical likelihood of both outcomes for youth. It therefore follows that justice-involved youth as a population have a higher rate of mental health issues compared to the population of community youth because the risk factors that predisposed them to their criminal offending are likely to have predisposed them to mental health issues as well.

Several shared risk factors have been identified in the literature. For example, in a large meta-analysis of 114 studies, Fowler, Tompsett, Braciszewski, Jacques-Tiura, and Baltes (2009) found a moderate effect size between exposure to community violence and externalizing problems among youth. A small but significant effect size was also found between exposure to community violence and internalizing problems among adolescents. Exposure to community violence is also a risk factor for the onset and maintenance of 
violent offending among male youth (Baskin \& Sommers, 2014, 2015). Childhood maltreatment is another salient example of a risk factor that can contribute to the onset of mental health issues and criminal offending among youth. There is robust evidence for the relationship between early childhood physical abuse and externalizing behavioural disorders, as well as the relationship between early childhood physical abuse and violent offending (e.g., Fox, Perez, Cass, Baglivio, \& Epps, 2015; Hawkins et al., 2000; Moylan et al., 2010; Maas, Herrenkohl, \& Sousa, 2008; Norman et al., 2012; Peltonen, Ellonen, Larsen, \& Helweg-Larsen, 2010; Silva, Graña, \& González-Cieza, 2014). Similarly, there is robust evidence that early childhood sexual abuse is a risk factor for the onset of affective disorders, and for the onset of sexual offending among youth (e.g., Browne \& Finkelhor, 1986; Hornor, 2010; Lindert et al., 2014; Maniglio, 2015; McCuish, Cale, \& Corrado, 2015; Morais, Alexander, Fix, \& Burkhart, 2018; Papalia, Ogloff, Cutajar, \& Mullen, 2018; Romano \& De Luca, 2001; Seto \& Lalumière, 2010).

The shared risk explanation is helpful in understanding group-level differences between justice-involved youth and community youth. However, it does not address the specific interplay between mental health issues and criminal offending among justiceinvolved youth (Development Services Group, 2017). The shared risk explanation also does not fully explain differences in the mental health profiles observed between youth who commit sexual offences and those who commit non-sexual offences. Based on shared risk, there should be similar rates of psychopathology comparing youth who commit sexual offences and those who commit non-sexual offences. This is because both groups of justice-involved youth possess risk factors that increase the statistical likelihood of criminal offending and the onset of mental health issues. The shared risk 
explanation is partially supported by the findings from Seto and Lalumière's (2010) meta-analysis that there were no significant differences between youth who committed sexual offences and those who committed non-sexual offences for general psychopathology, depression, psychotic symptoms, or suicidal tendencies. However, significant differences were found for anxiety and low self-esteem. These findings suggest the shared risk explanation is not sufficient to explain differences in psychopathology between youth who commit sexual offences and those who commit non-sexual offences. It is likely there are unique factors regarding how youth who commit sexual offences are differentially processed through the youth criminal justice system that account for some of these observed differences in psychopathology.

Causal mechanism. To determine the exact relationship between mental health issues and criminal offending among youth, researchers have investigated whether a causal mechanism exists. To date, there is limited empirical evidence for a causal association between mental health problems and criminal behaviour (Girard \& Wormith, 2004). For example, studies have consistently found mental health issues are generally not related to recidivism among youth. Schubert, Mulvey, and Glasheen (2011) used data from the longitudinal Pathways to Desistance Study (www.pathwaysstudy.pitt.edu) and found that having a mental health issue, except a substance use disorder, did not predict re-arrest in a sample of 797 male youth and 152 female youth. Further, after controlling for criminogenic risk factors, youth with a mental health issue, including a substance use disorder, were equally as likely to reoffend as youth without a mental health issue (Shubert et al., 2011). These findings are consistent with Skeem, Manchak, and Peterson (2011) who concluded a direct relationship between mental health problems and criminal 
offending exists for a minority of youth, but globally this association is almost fully accounted for by criminogenic risk factors.

Adding to the above evidence is work by Davis, Peterson-Badali, and Skilling (2015) who conducted an evaluation of a Canadian youth mental health court program. In their study, youth were referred to mental health court if they had a pre-existing mental health issue, if mental health issues were suspected, or if mental health issues were thought to contribute to their current criminal charges. Although mental health problems were prominent among the sample ( $82 \%$ had at least one prior diagnosis), only one in five youth had charges directly related to their mental health problems. This finding could be explained by the relatively low rate of externalizing disorders $(11.50 \%)$ in the sample, which evidence suggests is the cluster of mental health disorders most directly related to criminal behaviour. In a recent meta-analysis by Wibbelink, Hoeve, Stams, and Oort (2017) a small overall effect size $(d=0.36)$ between any mental health diagnosis and recidivism among justice-involved youth is reported. More specifically, no relationship was found between having an internalizing disorder (e.g., anxiety or depression) and recidivism $(d=0.02)$, whereas a small effect size is reported between having an externalizing disorder and recidivism $(d=0.42)$. When examining specific externalizing diagnoses, a moderate effect size is reported for conduct disorder $(d=0.55)$, whereas negligible effect sizes are reported for substance use disorder $(d=0.19)$, attention-deficithyperactive-disorder (ADHD; $d=0.38)$, and oppositional defiant disorder (ODD; $d=$ 0.08). Overall, it can be concluded that a causal relationship between internalizing spectrum disorders and criminal offending almost certainly does not exist, whereas a causal relationship between externalizing spectrum disorders and criminal offending 
likely exists for a sub-set of youth. Given this conclusion, the question remains: why are internalizing spectrum problems at least twice as high among justice-involved youth compared to the general population (Fazel, Doll, \& Långström, 2008)?

\section{Exacerbation Effect of the Criminal Justice System}

The research emphasis on elucidating the specific relationship between mental health problems and criminal offending behaviour among youth may have contributed to the under-prioritization of research regarding the effect of criminal justice system involvement on the mental health problems of justice-involved youth. Drawing from the shared risk explanation, if youth who criminally offend are statistically more likely to be predisposed to mental health problems, it follows that involvement in a high-stress and socially isolating system would likely contribute to the manifestation of such mental health problems. This mechanism is most likely true for internalizing spectrum problems, given there is very limited empirical evidence of a causal mechanism between internalizing disorders and criminal offending, but there is some evidence of shared risk factors between internalizing spectrum disorders and criminal offending (Fazel, Doll, \& Långström, 2008).

Researchers and practitioners have acknowledged the potential exacerbation effect of criminal justice system involvement on the mental health of justice-involved youth. For example, in their discussion regarding access to mental health care among justice-involved youth, Kutcher and McDougall (2009) state: "It is well-appreciated that the prison environment may lead to or exacerbate mental health problems...Additionally, separation from social supports may cause further stresses that in the presence of toxic environmental pressures may increase the risk or exacerbation of mental health problems 
in vulnerable youth" (p. 16). Despite the intuitive validity of this argument, few empirical studies have directly examined whether negative changes in mental health occur as youth enter and/or progress through the criminal justice system.

There is a dearth of information regarding how mental health problems change as youth progress through the criminal justice system. A handful of studies have examined changes in mental health among detained youth. For example, in a Canadian study by Cesaroni and Peterson-Badali (2010), justice-involved youth with a greater number of pre-existing vulnerabilities (e.g., child protection service involvement, witness to community violence, school problems) were significantly more likely to experience psychosocial adjustment difficulties while in custody compared to justice-involved without pre-existing vulnerabilities. These findings are consistent with similar work from the United Kingdom by Lennox and colleagues (2013) who investigated changes in mental health among male youth over their first 6-months in a detention facility. The proportion of youth with any mental health problem did not change over time, however there was a reduction in symptom severity among some youth. Another large-scale example is the Northwestern Juvenile Project (NJP; Teplin et al., 2002; 2012; 2015), which is a large prospective longitudinal study of youth detained at the Cook County Juvenile Temporary Detention Centre between 1995 and 1998. Youth were assessed upon entry into the detention facility, and approximately five years after baseline. The results of this project show mood and anxiety disorders decreased significantly post-detention, suggesting the prevalence of these disorders among detained youth may be in response to the detention itself. 
Unfortunately, as with most studies examining mental health problems among detained youth, the baseline assessment for the NJP was conducted after the youth were already detained. It is therefore not possible to know the proportion of baseline mental health problems that were pre-existing or are attributed to criminal justice system involvement leading up to detention. Further, findings based solely on detained samples of youth are not necessarily generalizable to justice-involved youth who have not experienced detention, either because of they are lower-risk or live in jurisdictions where the use of detention is less common (e.g., Canada).

\section{Current Study}

The current study examines self-reported changes in mental health attributed to the adjudication period of the youth criminal justice system process. Self-esteem is included as a mental health construct in the current study, and is relevant to examine in the context of youth criminal justice system involvement for several reasons. First, selfesteem is considered a feature of global psychological well-being (Mann, Hosman, Schaalma, \& de Vries, 2004; McClure et al., 2010; Zimmerman, Copeland, Shope, \& Dielman, 1997). As such, there is as much an imperative to address poor self-esteem as any other mental health issues present among justice-involved youth. This is not to say other constructs related to well-being are not valid targets to study and potentially address, including self-determination, autonomy, or competence; however, self-esteem was prioritized because of the existing evidence that adolescence is a particularly vulnerable developmental period for self-esteem. Specifically, in a review of the developmental life-course of self-esteem, Orth and Robins (2014) identified late adolescence as a critical period in the trajectory of self-esteem because it is the period 
when self-esteem crystallizes. Self-esteem among male youth typically increases in late adolescence, and then becomes a stable trait until the mid-50s (Donnellan, Kenny, Tresniewski, Lucas, \& Conger, 2012; Greve \& Enzmann, 2003; Kuster \& Orth, 2013). Given the critical period for self-esteem aligns with the same developmental window that most justice-involved youth initially become involved in the youth criminal justice system, it is important to prioritize self-esteem in youth justice research so we can better understand whether the developmentally normative upward trajectory of self-esteem is disrupted by involvement in the youth criminal justice system.

Self-esteem was also prioritized above other constructs of youth well-being in the current study because poor self-esteem is consistently linked to negative outcomes among justice-involved youth making it a priority target for the current study. For example, low self-esteem is associated with increased psychopathology among justice-involved youth (Donnellan, Trzesniewski, Robins, Moffit, \& Caspi, 2005). In a study by Van Damme, Colins, and Vanderplasschen (2014), detained male youth with low self-esteem were significantly more likely to have psychiatric disorders compared to those with high selfesteem. Specifically, $18.0 \%$ of youth with low self-esteem were diagnosed with an internalizing disorder compared to $2.0 \%$ of youth with high self-esteem. Similarly, $41.0 \%$ of youth with low self-esteem were diagnosed with an externalizing disorder compared to $14.0 \%$ of youth with high self-esteem (Van Damme et al., 2014).

There is also some evidence self-esteem is negatively associated with custody placements among justice-involved youth. For example, previous work indicates incarcerated youth have significantly lower self-esteem compared to non-justice involved youth (Forrest, Tambor, Riley, Ensminger, \& Starfield, 2000). Low self-esteem is also 
considered a risk factor for poor institutional adjustment among justice-involved male youth (Cesaroni \& Peterson-Badali, 2005; Greve, Enzmann, \& Hosser, 2001). In a more recent study by Imbach and colleagues (2013), low self-esteem was significantly associated with greater offence severity. A possible explanation for this observed relationship is that youth who commit more severe criminal offences receive more severe correctional responses, which in turn leads to greater declines in self-esteem. Youth who commit more severe offences also stay in the youth criminal justice system for longer. It is therefore possible the findings from Imbach and colleagues (2013) are explained by length of time in the system. The potential association between length of time in the youth criminal justice system and poor self-esteem is the basis for hypothesizing the magnitude of change in reported self-esteem will be associated with length of adjudication in the current study (see below).

Using a retrospective pre-post design, youth were asked to report how they felt prior to being involved in the criminal justice system and since being involved in the criminal justice system. This type of study design enables the examination of pre- to postcharge differences, as well as group comparisons based on offence type. Comparisons based on offence type, specifically comparisons regarding youth who committed sexual offences versus those who committed non-sexual offences, are particularly important when examining associated effects of criminal justice system involvement with the internal states of youth. Previous work indicates youth who commit sexual offences often report heightened emotional states (e.g., shame and stigma) while in the criminal justice system which are attributed to the labelling they experience due to the sexual nature of their offending (Bonnar-Kidd, 2010; Geer, 2008; Harris \& Socia, 2016; Harris, Walfield, 
Shields, \& Letourneau, 2016; Letourneau \& Caldwell, 2013; Robbers, 2009; Sapp \& Vaughn, 1990). Several theoretical models of sexual offending also emphasize the role of psychopathology and self-esteem in the onset of sexual offending (Kafka, 1997; Miner et al., 2010; Ward \& Seigert, 2002). Identifying the timing of psychopathology among youth who have sexually offended (i.e., pre- or post- criminal justice system involvement) has important implications for etiological theories of youth sexual offending that emphasize the role of affective dysregulation and emotional incompetence. Among other negative effects that may be associated with criminal justice system involvement unique to youth who sexually offend is greater social isolation. Youth who commit sexual offences are at greater risk for being socially rejected due to their status as someone who has sexually offended, and are more likely to self-isolated because of the felt stigma associated with sexual offending (Human Rights Watch, 2013; Letourneau et al., 2018). This is consistent with the findings from Seto and Lalumière's meta-analysis where youth who committed sexual offences were significantly more likely to be socially isolated compared to youth who committed non-sexual offences. Social isolation is negatively associated with positive psychological development among youth, and can ultimately increase distress as it often leads to self-imposed restricted access to mental health and social support (Collins \& Steinberg, 2006; Miller, Esposito-Smythersm, Leichtweism, 2015; Safren \& Pantalone, 2006). As such, changes in perceived social support were also examined in the current study between youth who committed sexual offences and those who committed non-sexual offences.

Hypotheses. The current study is driven by three research hypotheses: 1) a negative change in mental health symptoms, including anxiety, depression, and self- 
esteem, and perceived social support will be observed across all youth in the sample; 2) the magnitude of reported change will be greater for youth who committed a sexual offence compared to youth who committed a non-sexual offence; and, 3) the magnitude of reported change will be related to the total time since youth were charged for all youth.

\section{Methods}

\section{Participants}

The study sample includes 90 male youth who completed the research protocol. To be eligible for the study, youth participants had to identify as male. The decision to only include male youth was primarily driven by the study's focus on differences between youth who commit sexual offences and those who commit non-sexual offences. Most theoretical models of youth sexual offending, and most research on youth sexual offending, focus on male youth because males commit the majority of sexual offences (Varma \& Leroux, 2019). Decades of feminist pathways to crime research has also demonstrated females enter and experience the criminal justice system in unique ways compared to males, and require gender-specific criminal justice system responses (Blanchette \& Brown, 2008; Comack, 1996, 2006; Fortin, 2004; Hannah-Moffat, 2004; Jones, Brown, Wanamaker, \& Greiner, 2014; Laishes, 2002; Pollack, 2007; Renzetti, 2013). Taken together, it was decided the most appropriate approach would be to only include males as the orientation and empirical foundation of the current study could not adequately capture gender-specific factors and nuances.

Other eligibility criteria include: youth must have been charged for a criminal offence they committed between the ages of 12 and 17 years old; youth must have been referred for a court-ordered mental health assessment; and, youth must have attended 
their appointment from a community-based living situation. Youth who required an English language interpreter were not eligible to participate.

Recruitment. Participant recruitment was conducted in the Youth Justice Clinic at the Centre for Addiction and Mental Health (CAMH) in Toronto, ON, and the Family Court Clinic at the Royal in Ottawa, ON. Primary research ethics approval was granted by the CAMH Research Ethics Board (REB) for recruitment in the Youth Justice Clinic, and primary research ethics approval was granted by the Royal REB for the recruitment in the Family Court Clinic. The Carleton University REB also conducted an administrative review of both protocols and granted approval for each recruitment site.

Recruitment in both clinics took place over the course of 26 months between October 2016 and December 2018. A total of 125 eligible youth were approached during the recruitment period, with 91 agreeing to participate in the protocol (73\% recruitment rate). The research team tracked the primary reasons youth declined to participate in the research protocol. In many instances (35\%), eligible youth wanted to complete the research protocol but were under the REB-approved age of consent. In the current study, youth under the age of 16 years old needed consent from their legal guardian to participate in the protocol. This was a barrier for youth who attended their appointments with child protection workers, temporary foster parents, group home workers, or open custody workers. Although the age of consent was a barrier for many youth in the current study, the tracking of this information is important for future work as it highlights the need to either modify the age of consent for research, or modify the definition of guardian, for research with justice-involved youth who are more likely to be in the child 
welfare system. This data will be used in future work to advocate for greater accessibility to research for justice-involved youth.

Another barrier to participation was the accessibility of the clinics and the length of the assessment appointments. Most assessment appointments took the full day, and youth and caregivers often travelled long distances to attend their appointments (particularly in Ottawa where the referral catchment area is very large). Approximately $15 \%$ of youth declined to participate because they, their caregivers, and/or their workers were not available to stay past their scheduled appointment time. Approximately $20 \%$ of youth requested to participate in the research at a second appointment, but either did not attend their second appointment or their second appointment was cancelled by their clinician. Another related barrier was that $10 \%$ of youth who wanted to participate and attended their second appointment did so from secure detention, meaning they either breached their conditions or committed a new offence between the time of being agreeing to the research and their second appointment.

Some potential youth participants (15\%) also left their assessment appointments without notifying the research team. This often occurred after youth became emotionally dysregulated in their clinical assessments. Finally, only $5 \%$ of youth explicitly said they were not interested in participating in the research protocol.

Sample descriptives. The mean age of study youth at the time of their index offence was $16.8(S D=1.6)$ years, and their mean education level was Grade $11(S D=$ 1.5). Most youth identified as Black (45.2\%) followed by White (32.4\%), with the remaining youth identifying as Indigenous (9.5\%), Asian (4.8\%), Latin American (3.6\%), and Arabic (3.6\%). The ethnicity categories presented here align with Statistics Canada 
Census program variables (Statistics Canada, 2019). It is also important to note the ethnicity breakdown of the current sample does not reflect the ethnicity breakdown of the recruitment cities (Ottawa and Toronto).

Almost all youth were living at home at the time of their assessment (64.4\%), with $9.2 \%$ living with other family members, $2.3 \%$ living with foster parents, $11.5 \%$ living in a group home, and 10.3\% living in an open custody facility. Most youth (63.7\%) reported experiencing some form of maltreatment at home, with approximately $50 \%$ reporting a history of verbal abuse, $25 \%$ reporting a history of emotional abuse, $20 \%$ reporting a history of physical abuse, $10 \%$ reporting a history of neglect, and $3 \%$ reporting a history of sexual abuse. Additionally, 30\% of youth reported witnessing intimate partner violence at home. Half of the youth (50\%) were involved with child protective services prior to their involvement in the criminal justice system, with $20 \%$ reporting a history of removal from the home.

Approximately one-third (27.8\%) of youth were charged with a sexual offence, and the remaining youth $(72.2 \%)$ were charged with a non-sexual offence. The sexual offence group were court-ordered for a mental health assessment based on a sexual index offence defined in the Canadian criminal code. All youth in the sexual offence group had been charged with a contact sexual offence, except for one who was exclusively charged with possession of child pornography. Approximately two-thirds (65\%) of the youth charged with a contact sexual offence committed a sexual offence against a child, defined as under the age of 12 years old and at least three years younger than the accused. The remainder of those charged with a contact sexual contact offence committed a sexual offence against a peer, defined as between the ages of 12 and 18 years old. The non- 
sexual offence group were court-ordered for a mental health assessment based on a violent index offence defined by the Canadian criminal code. Examples include aggravated assault, assault with a weapon, robbery, and unlawful possession of a weapon. All youth in the non-sexual offence group had at least one offence against a person. As such, both groups were composed of justice-involved youth who had committed serious offences based on existing Criminal Code legal interpretations of offence seriousness. Table 5 presents descriptive information for the sexual and non-sexual offence groups of youth included in the current sample. The groups did not significantly differ regarding age, or education grade level. There was a significant difference in the proportion of both groups who were White. Specifically, the majority of youth who committed a sexual offence were White $(63.6 \%)$, whereas the majority of youth who committed a non-sexual offence were non-White $(65.4 \%)$. The groups of youth did not significant differ in terms of previous history with child protection services, or across any of the maltreatment history variables. 
Table 5.

Descriptive information by offence type

\begin{tabular}{|c|c|c|c|c|}
\hline & $M(\mathrm{SD}) / \%$ & $M(\mathrm{SD}) / \%$ & $t$-value $/ \chi^{2}$ & $d$ \\
\hline & $\begin{array}{l}\text { Sexual Offence } \\
\qquad(N=25)\end{array}$ & $\begin{array}{c}\text { Non-Sexual } \\
\text { Offence } \\
(N=65)\end{array}$ & & \\
\hline Age & $17.12(2.4)$ & $16.69(1.1)$ & 0.85 & -0.27 \\
\hline Grade & $10.68(1.6)$ & $10.90(1.4)$ & -0.61 & 0.15 \\
\hline $\begin{array}{l}\text { Ethnicity } \\
\text { White } \\
\text { Non- } \\
\text { White }^{\mathrm{a}} \\
\text { Indigenous }\end{array}$ & $\begin{array}{c}63.6 \\
31.8 \\
4.5\end{array}$ & $\begin{array}{c}25.0 \\
65.4 \\
9.6\end{array}$ & $9.96^{*}$ & --- \\
\hline Child protection & 60.9 & 46.2 & 1.47 & 0.35 \\
\hline \multicolumn{5}{|c|}{ Maltreatment history } \\
\hline Sexual abuse & 8.3 & 1.6 & 2.38 & 0.93 \\
\hline Physical abuse & 20.8 & 19.0 & 0.04 & 0.09 \\
\hline Verbal abuse & 47.8 & 50.0 & 0.03 & 0.05 \\
\hline Emotional abuse & 21.7 & 27.0 & 0.24 & 0.14 \\
\hline Neglect & 11.1 & 11.5 & 0.002 & 0.05 \\
\hline Witness violence & 36.0 & 38.5 & 0.05 & 0.05 \\
\hline \multicolumn{5}{|l|}{ Index offence } \\
\hline Substance use & 29.2 & 47.6 & 2.42 & 0.46 \\
\hline Weapon use & 4.2 & 53.1 & $17.47^{* * *}$ & 1.74 \\
\hline
\end{tabular}


There was no significant difference between groups in terms of substance use at the time of the index offence. Overall, a substantial proportion of both groups of youth reported being under the influence of either alcohol or drugs at the time of their index offence. There was a significantly large difference in the proportion of groups who used a weapon during the index offence. Specifically, only $4.2 \%$ of youth who committed a sexual offence used a weapon compared to $53.1 \%$ of youth who committed a non-sexual offence.

\section{Measures}

Summary sheet. Youth were asked to provide summary details of their index offence, defined as the offence that resulted in their referral for a court-ordered mental health assessment. These details were collected using a standardized summary sheet (Appendix C), and included brief participant details (e.g., age at time of index offence), index offence details (e.g., type of offence, age and gender of the victim, if a co-accused was involved), and adjudication details (e.g., was the youth in Youth Mental Health Court, length of time since index arrest). Youth were also asked about any other criminal justice system involvement. This information included any arrests, charges, or administrative breaches that occurred either before or after the youth was charged with the index offence. The summary sheet was also used to identify adolescents who had committed a sibling incest offence, as this sub-group of participants was going to be used for a set of incest-specific research studies published outside of the current dissertation. Given the often cyclical nature of criminal justice system involvement, capturing this information verbally was required to account for the large variability in experience. A 
coding form was developed to input the summary sheet information into an electronic database (Appendix D).

Background information. Demographic information including age, ethnicity, and school grade was collected. Other background information collected covered three domains: family background, maltreatment history, and index offence details (Appendix E).

Family background. Youth were asked about the number of siblings they have, the type of siblings they have (e.g., younger biological sister, older non-biological brother), whether they lived with their biological parents, and about all adults living in their household at the time of the index offence (e.g., biological grandmother, nonbiological father). Youth were also asked about their living situation. Specifically, they were asked about where they were currently living, where they were living at the time of the offence, and if they had ever been removed from their home. Family background information also included if and how often the adults at home used illegal drugs, used alcohol, were violent toward each other, and how often the police came to the household.

Maltreatment history. Youth were asked if and how often they were neglected, and verbally, physically, and emotionally abused, at home. Youth were also asked if and how often their siblings were neglected, and verbally, physically, and emotionally abuse, at home. Youth were also asked to identity who had abused them (e.g., biological mother, non-biological father).

Index offence details. Youth were asked how they felt at the time of offence, if they were under the influence of substances, and if they used a weapon. Youth were also asked about the age, gender, and relationship to the index offence victim(s). 
Revised Child Anxiety and Depression Scale. Anxiety and depression were measured using a modified version of the Revised Child Anxiety and Depression Scale Short version (RCADS-25; Appendix F) developed by Chorpita, Moffit, and Gray (2005). The RCADS-25 is a 25-item measure that assesses DSM-oriented anxiety and major depression. Items are rated on a four-point Likert scale ranging from "never" to "always". The RCADS-25 yields three subscales: total anxiety, total depression, and total anxiety and depression (Chorpita, Yim, Moffit, Umemoto, \& Francis, 2000). Items from each subscale are summed, with higher scores reflection greater anxiety and depression problems.

The RCADS-25 was chosen because it is a brief measure with clinical cut-off scores, and is freely available by the authors. It has also been validated for use with school-based and clinical samples of youth, which aligns with the current study sample. For example, in a study conducted by Ebesutani and colleagues (2012) the 15-item anxiety subscale discriminated between youth with and without a DSM anxiety diagnosis. The 10-item depression subscale also discriminated between youth with and without DSM major depression or dysthymic disorder.

The RCADS-25 items were modified so youth rated each item based on how they felt since they were charged (e.g., "Ever since I was charged, I have trouble sleeping"), and before they were charged (e.g., "Did you have trouble sleeping before you were charged?"). As such, each item was rated twice which allows for the calculation of precharge scores, post-charge scores, and change scores. Pre- and post-charge RCADS-25 anxiety, depression, and total raw scores, as well as T-scores were calculated using the scoring algorithm provided by the authors (https://www.childfirst.ucla.edu/resources/). 
Rosenberg Self-Esteem Scale. Self-esteem was measured using a modified version of the Rosenberg Self-Esteem Scale (RSES; Appendix G) developed by Rosenberg (1998). The RSES is a 10-item measure rated on a four-point Likert scale ranging from "strongly agree" to "strongly disagree". All items are summed, with higher scores representing greater self-esteem.

The RSES was chosen because it is a brief measure that assesses global selfesteem, and is freely available by the authors. The RSES was also originally developed for high school students which aligns with the current study sample. For example, in a review of the RSES psychometric properties for youth, Gray-Little, Williams, and Hancock (1997) report internal consistency ranging from .72 to .88 across studies. The RSES has also been used to measure self-esteem specifically among justice-involved youth, and among youth who have specifically committed a sexual offence (Donnellan, et al., 2005; Monto, Zgourides, \& Harris, 1998).

The RSES items were modified so youth rated each item based on how they felt since they were charged (e.g., "Ever since I was charged, I feel like I am failure"), and before they were charged (e.g., "Did you feel like a failure before you are charged"). As such, each item was rated twice which allows for the calculation of pre-charge scores, post-charge scores, and change scores.

Multidimensional Scale of Perceived Social Support. Perception of social support was measured using a modified version of the Multidimensional Scale of Perceived Social Support (MSPSS) developed by Zimet, Dahlem, and Zimet (1988; Appendix H). The MSPSS is a 12-item measure rated on a seven-point Likert scale ranging from "very strongly agree" to "very strongly disagree". The items of the MSPSS 
measure perceived support across three subscales: family, friends, and significant others. Total and subscale scores are calculated by averaging the items associated with each respective subscale, with higher scores representing greater perceived support.

The MSPSS was chosen because it is a brief measure, and is freely available by the authors. The MSPSS has also been specifically validated for use with youth (Zimmet, Powell, Farley, Werkman, \& Berkhoff, 1990). For example, in a sample of urban youth, the family, friends and significant other subscales yielded Cronbach coefficients of .91, .89, and .91 respectively (Canty-Mitchell \& Zimet, 2000). More recently, the MSPSS has been used to measure perceived social support among registered and non-registered youth who committed sexual offences in the United-States (Letourneau et al., 2018).

The MSPSS items were modified so youth rated each item based on how they felt since they were charged (e.g., "Ever since I was charged, my family really tries to help me"), and before they were charged (e.g., "Did your family really try to help you before you were charged?"). As such, each item was rated twice which allows for the calculation of pre-charge scores, post-charge scores, and change scores. In the current sample.

\section{Procedure}

All participants who agreed to participate in the research protocol were given a unique participant ID. This ID was used to link information from the summary sheets, survey, and interview.

Consent to participate in the research protocol for participants 16 years and older was obtained prior to collecting any information. For youth under the age of 16, consent to participate in research was obtained by their legal guardian after the research team received verbal confirmation of their interest in participating. Youth participants under 
the age of 16 years old then signed an assent form prior to collecting any information. Legal guardians were not permitted to sit-in on the research protocol with their youth, and were not provided with any information regarding their youth's responses.

The information contained in the consent and assent forms was described, and participants were given time to read the forms. Because of the legalistic language used in the consent forms, the research team used simplified language to describe the information, particularly for younger participants. Given the sensitive nature of some of the questions asked, the research team also re-iterated and emphasized the limits of confidentiality during the consent process. Consent and assent forms from both recruitment sites are in the appendix (Appendix I and Appendix J).

Once consent and/or assent was obtained, participants provided verbal answers for the summary sheet. To ensure consistent verbal information was collected at this stage of the protocol, a training guide was developed and implemented (Appendix C). The purpose of collecting this information verbally was to facilitate the accurate documentation of complicated criminal histories, and to build rapport with the youth ahead of the interview.

After the summary sheet information was collected, youth answered the survey. The survey was administered using a laptop and E-prime software. Although E-prime is typically used for response-time experimental tasks, it is one of the few software programs that can run a survey without being connected to the Internet. Choosing a survey platform that did not rely on the Internet was important to ensure survey stability in the clinics, where wireless Internet connections can be unreliable. It was also important not to rely on the Internet as it was expected some youth would have bail conditions that 
restricted Internet access. The E-prime software was also chosen because it supports embedded audio files with each question. All survey questions had a corresponding automated voice recording, which allowed youth to listen to the questions using headphones. Giving youth participants the option of listening to the questions increased the overall accessibility of the research protocol, including increased question comprehension for youth where English was their second language. It also helped mitigate expected fatigue, particularly after the psychoeducational testing component of the youth's clinical assessment. Overall, approximately $15 \%$ of youth listened to the questions. The survey measures were counter-balanced, and the average time it took youth to complete it was 45 minutes.

Youth received $\$ 50$ as compensation for their participation and were asked to initial a confirmation of compensation document for record keeping purposes (Appendix K). Before leaving, youth were given a debriefing form which included contact information for local mental health services (Appendix L and Appendix M). They were also asked to provide any feedback about the research questions or research protocol. The research team also assessed the youth's emotional state prior to letting them leave the clinic. If they seemed distressed or dysregulated, the researcher notified the supervising clinician. This occurred twice during the recruitment period.

\section{Results}

To test the first hypothesis, a series of paired samples $t$-tests was conducted to examine pre- to post-charge change in reported anxiety, depression, self-esteem, and social support. These analyses were conducted with the full sample of youth, and then the two sub-samples of youth who had committed a sexual offence and those who had 
committed a non-sexual offence. To test the second hypothesis, a series of independent samples $t$-tests, was conducted comparing the pre-charge scores between the two subsamples of youth, and post-charge scores respectively. An alternative statistical approach would have been to conduct a mixed ANOVA using within- and between-subject factors. However, conducting $t$-tests is an equivalent approach when there are only two time points (pre- and post-charge), and arguably better accommodates unequal and relatively small group sizes which is the case in the current study (Field, 2018). Further, the main advantage of using an ANOVA instead of $t$-tests is to reduce Type I error; however, this advantage is not relevant to current analysis as pre- and post-charge simple slope $t$-tests would have still needed to be conducted if the ANOVA been used. Overall, although a mixed ANOVA would have been an acceptable statistical approach, the current use of $t$ tests is equally acceptable, and a more parsimonious approach.

Pre- to post-charge change scores were also calculated and examined using independent samples $t$-tests. Finally, simple linear regression was used to test the relationship between pre- to post-charge change scores and total length of time since the index offence.

Cohen's $d$ was calculated for all paired and independent samples $t$-tests using G*Power (Faul, Erdfelder, Lang, \& Buchner, 2007, 2009). For the paired samples $t$-tests a negative Cohen's $d$ reflects a negative change in pre-charge to post-charge scores. For the independent samples $t$-tests a negative Cohen's $d$ means the youth who committed a sexual offence reported worse scores compared to the youth who committed a non-sexual offence. As per Cohen's (1988) suggested interpretation, an effect size of 0.30 is considered small, 0.50 is considered medium, and 0.80 is considered large. 


\section{Anxiety}

Table 6 presents the paired samples $t$-test comparisons of pre- and post-charge reports of anxiety. A significant negative change was found for the full sample of youth. A significant negative change was also found for both sub-samples of youth, however, a medium effect size was observed for youth who committed a sexual offence $(d=-0.68)$, whereas a small effect size was observed for youth who committed a non-sexual offence $(d=-0.34)$.

Figure 2 presents the pre- and post-charge reported anxiety means for both subsamples of youth to visualize change over time. The magnitude of change experienced by the two sub-samples of youth was investigated by calculating and examining pre- to postcharge change scores with higher scores reflecting greater anxiety scores post-charge. The mean change score for youth who committed a sexual offence was $5.96(S D=8.74)$ and the mean change score who committed a non-sexual offence was 2.14 (6.23). The difference between the anxiety change scores was significant, $t(87)=2.31, p=.02$. Cohen's $d$ indicates a medium effect $(d=-0.54)$.

Pre- and post-charge simple slope independent samples $t$-tests were also conducted. Pre-charge reported anxiety did not significantly differ between youth who committed a sexual offence and those who committed a non-sexual offence, $t(87)=-0.45$, $p=.66$. A small effect size is reported for the pre-charge comparison, $(d=0.10)$, with youth who committed a non-sexual offence scoring in the problematic direction. Similarly, post-charge reported anxiety did not significantly differ between the two subsamples of youth, $t(87)=1.49, p=.14$. A small effect is reported for the post-charge 
comparison $(d=-0.35)$, with youth who committed a sexual offence scoring in the problematic direction.

Based on Figure 2, and the reported means, it is clear youth who committed a sexual offence experienced greater negative change in terms of reported anxiety compared to youth who committed a non-sexual offence.

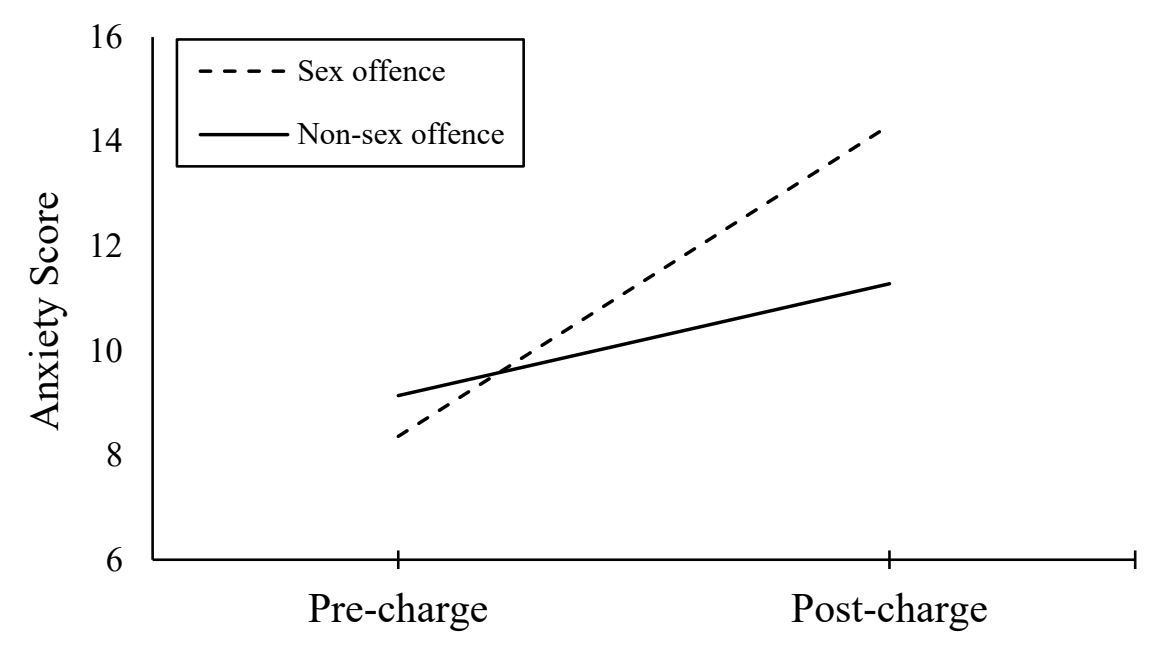

Adjudication Time Point

Figure 2. Pre- and post-charge mean reported anxiety scores for youth who committed sexual offences and youth who committed non-sexual offences.

The proportion of youth from both sub-samples who scored in the clinical range on the anxiety scale (T-score $>70)$ pre- and post-charge was also examined. Among youth who committed a sexual offence, $16 \%$ scored in the clinical range pre-charge and $36 \%$ scored in the clinical range post-charge. Among youth who committed a non-sexual offence, $13 \%$ scored in the clinical range pre-charge and $20 \%$ scored in the clinical range post-charge. This finding indicates the magnitude of negative change experienced by youth who committed sexual offences is more clinically severe. 


\section{Depression}

Table 6 presents the paired samples $t$-test comparisons of pre- and post-charge reports of depression. A significant negative change was found for the full sample of youth. A significant negative change was also found for youth who committed a sexual offence but not for youth who committed a non-sexual offence. A large effect size is reported for youth who committed a sexual offence $(d=-0.80)$, suggesting the reported increase in depression from pre- to post-charge is severe.

Figure 3 presents the pre- and post-charge reported depression means for both sub-samples of youth to visualize change over time. The mean depression change score was $4.28(S D=5.37)$ for youth who committed a sexual offence and $0.23(S D=5.23)$ for youth who committed a non-sexual offence. This difference was large and significant, $t(87)=3.26, p=.002, d=-0.77$.

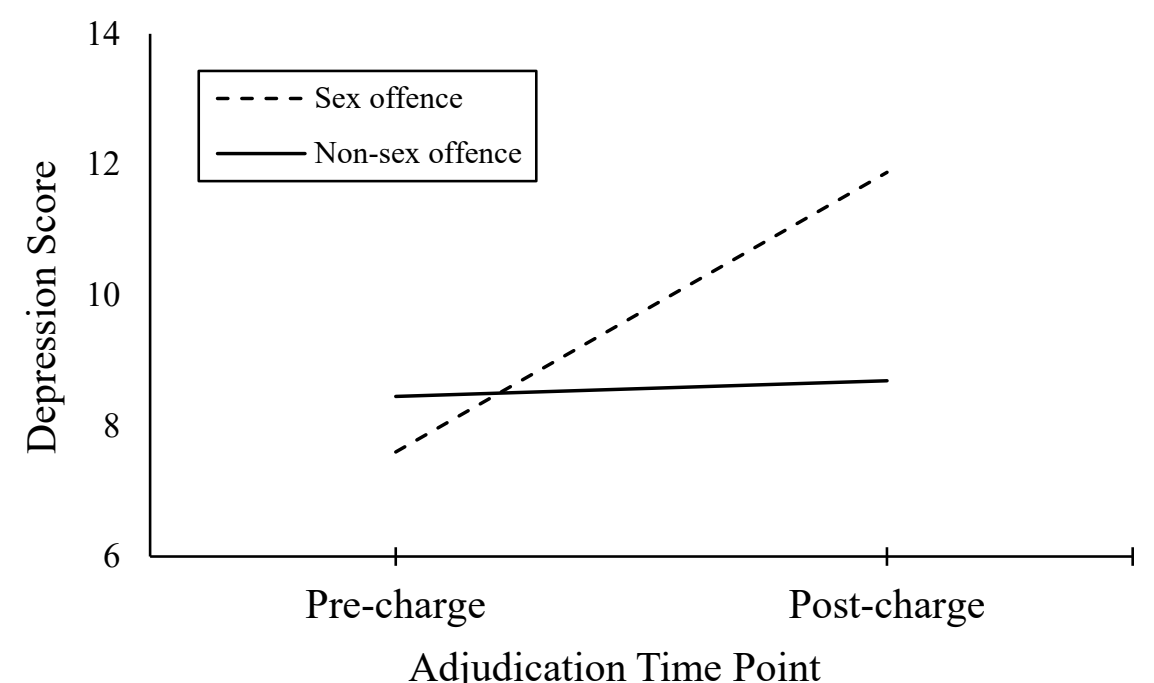

Figure 3. Pre- and post-charge mean reported depression scores for youth who committed sexual offences and youth who committed non-sexual offences. 
Pre- and post-charge simple slope independent samples $t$-tests were also conducted. Pre-charge reported depression did not significantly differ between youth who committed a sexual offence and those who committed a non-sexual offence, $t(88)=0.69$, $p=.49$. A small effect size is reported for the pre-charge comparison $(d=0.16)$, with youth who committed a non-sexual offence scoring in the problematic direction. Postcharge reported depression did significantly differ between the two sub-samples of youth, $t(87)=2.04, p=.04$. A medium effect size is reported for the post-charge comparison $(d=$ -0.48), with youth who committed a sexual offence scoring in the problematic direction. This suggests the degree of difference between groups in relation to depression symptoms widened over time.

The proportion of youth from both sub-samples who scored in the clinical range on the depression scale (T-score $>70)$ pre- and post-charge was also examined. Among youth who committed a sexual offence, $8 \%$ scored in the clinical range pre-charge and $28 \%$ scored in the clinical range post-charge. Among youth who committed a non-sexual offence, $11 \%$ scored in the clinical range pre-charge and $14 \%$ scored in the clinical range post-charge. The three and a half fold increase in the proportion of youth who committed a sexual offence and met the clinical threshold for depression indicates the magnitude of negative change experience by these youth is more clinically severe compared to youth who committed a non-sexual offence. 
Table 6

Reported changes in anxiety, depression, self-esteem, and social support, pre- and post-offence charge

\begin{tabular}{|c|c|c|c|c|c|c|}
\hline & & Pre-Charge & Post-Charge & & & \\
\hline & $N$ & Mean (SD) & Mean (SD) & $t$-value & $95 \% \mathrm{CI}$ & Cohen's $d^{a}$ \\
\hline \multicolumn{7}{|l|}{ Anxiety } \\
\hline Full sample & 89 & $8.92(7.41)$ & $12.13(8.71)$ & $-4.22^{* * *}$ & $-4.73,-1.70$ & -0.45 \\
\hline Sex offence & 25 & $8.36(8.01)$ & $14.32(9.91)$ & $-3.41^{* *}$ & $-9.57,-2.35$ & -0.68 \\
\hline Non-sex offence & 64 & $9.14(7.22)$ & $11.28(8.12)$ & $-2.75^{* *}$ & $-3.70,-0.58$ & -0.34 \\
\hline \multicolumn{7}{|l|}{ Depression } \\
\hline Full sample & 89 & $8.21(5.52)$ & $9.58(6.75)$ & $-2.3^{*}$ & $-2.54,-0.21$ & -0.25 \\
\hline Sex offence & 25 & $7.60(5.04)$ & $11.88(7.11)$ & $-3.99^{* * *}$ & $-6.49,-2.07$ & -0.80 \\
\hline Non-sex offence & 64 & $8.45(5.72)$ & $8.69(6.45)$ & -0.36 & $-1.54,1.07$ & -0.04 \\
\hline \multicolumn{7}{|l|}{ Self-esteem } \\
\hline Full sample & 88 & $25.26(4.19)$ & $22.45(3.84)$ & $5.41^{* * *}$ & $1.78,3.84$ & -0.58 \\
\hline Sex offence & 24 & $25.21(5.72)$ & $20.71(4.75)$ & $3.49^{* *}$ & $1.83,7.17$ & -0.71 \\
\hline Non-sex offence & 64 & $25.28(3.62)$ & $23.11(3.31)$ & $4.27^{* * *}$ & $1.15,3.19$ & -0.53 \\
\hline
\end{tabular}




\section{Social Support}

\begin{tabular}{cccccccc}
\hline Full sample & 89 & $4.32(1.34)$ & $4.40(1.34)$ & -0.76 & $-0.29,0.13$ & 0.08 \\
\hline Sex offence & 24 & $4.82(1.28)$ & $4.82(1.38)$ & 0.01 & $-0.55,0.55$ & -0.002 \\
\hline Non-sex offence & 65 & $4.14(1.32)$ & $4.25(1.30)$ & -1.03 & $-0.33,0.11$ & 0.13
\end{tabular}

Notes. ${ }^{a}$ Negative Cohen's $d$ means negative change mental health, meaning either an increase in depression or anxiety or a decrease in self-esteem or social support..

${ }^{*} p<.05,{ }^{* *} p<.01,{ }^{* * *} p<.001$ 


\section{Self-Esteem}

Table 6 presents the paired samples $t$-test comparisons of pre- and post-charge reports of self-esteem. A significant negative change was found for the full sample of youth, and the two sub-samples of youth, with medium to large effect sizes reported for all comparisons.

Figure 4 presents the pre- and post-charge reported self-esteem means for both sub-samples of youth to visualize change over time. Pre- to post-charge change scores were calculated, with higher scores reflecting greater negative change. The mean change score was $4.50(S D=6.32)$ for youth who committed a sexual offence and $2.17(S D=$ 4.07) for youth who committed a non-sexual offence, but this difference was not significant, $t(30.44)=1.68, p=.10, d=-0.48$.

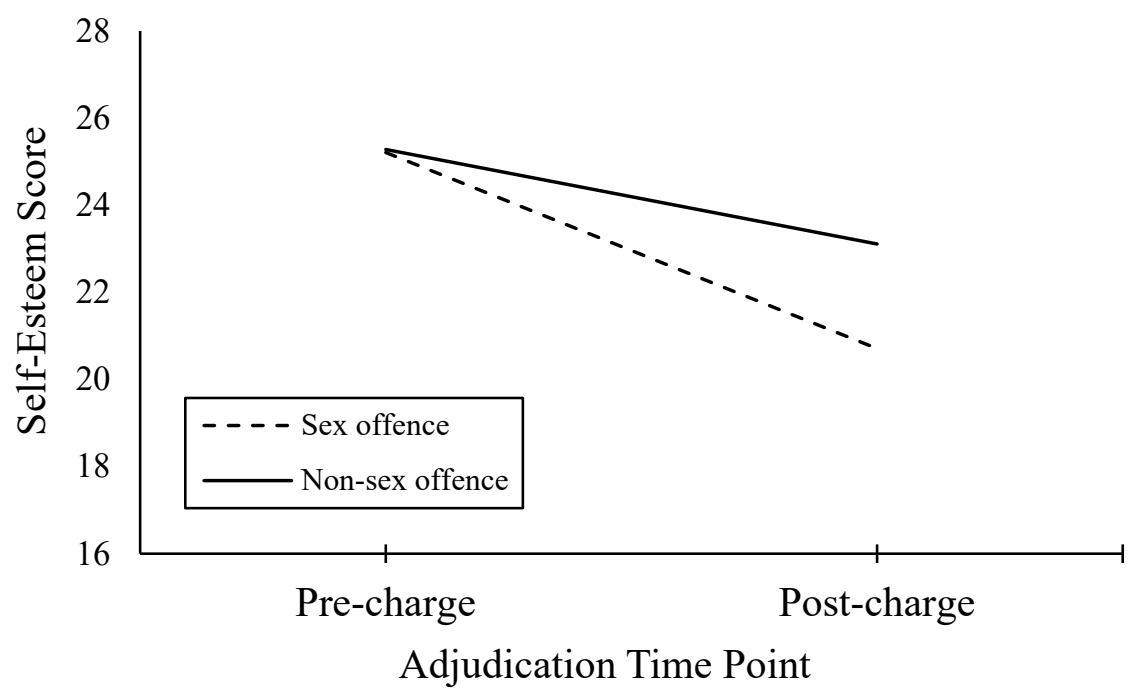

Figure 4. Pre- and post-charge mean reported self-esteem scores for youth who committed sexual offences and youth who committed non-sexual offences. 
Pre- and post-charge simple slope independent samples $t$-tests were also conducted. Pre-charge self-esteem scores did not significantly differ between youth who committed a sexual offence and those who committed a non-sexual offence, $t(87)=-0.11$, $p=.91, d=-0.03$. However, post-charge self-esteem scores did significantly differ between the two subsamples of youth, $t(86)=-2.33, p=.03, d=-0.65$.

\section{Social Support}

Table 6 presents the paired samples $t$-test comparisons of pre- and post-charge reports of social support. No significant negative change was found for the full sample youth, or for youth who committed a sexual offence and a non-sexual offence respectively. The effect sizes for these comparisons are negligible. Pre-charge social support scores did not significantly differ between youth who committed a sexual offence and those who committed a non-sexual offence, $t(88)=1.66, p=.10, d=0.39$. Post-charge social support scores also did not significantly differ between the two sub-samples of youth, $t(87)=1.80, p=.08, d=0.43$.

The social supports means reported in Table 4 are not very indicative of the overall perceptions of social support among the sample of youth because they correspond to the "neutral" anchor on the Multidimensional Scale of Perceived Social Support (MSPSS). As such, we categorized the social support scores into low support, moderate support, and high support as per the MSPSS scoring guidelines (Zimet et al., 1988). Precharge, there was a significant difference in the proportion of youth who committed a sexual offence and reported high support compared to youth who committed a non-sexual offence, $X^{2}(2, N=88)=7.67, p=0.02$. Specifically, $52.0 \%$ of youth who committed a sexual offence reported high levels of social support pre-charge, compared to $22.2 \%$ of 
youth who committed a non-sexual offence. Instead, most youth who committed a nonsexual offence reported moderate social support (63.5\%). Equal proportions of youth who committed a sexual offence and those who committed a non-sexual offence reported low levels of support pre- charge (14.3\% and $12.0 \%$, respectively).

Post-charge, there was no significant difference in the proportions of youth from both groups within each of the social support categories, $X^{2}(2, N=87)=5.67, p=0.06$. This finding is because the proportion of youth who committed a sexual offence and reported high levels of support was reduced at the post-charge stage, thereby creating a more equal distribution of cases across social support categories. Specifically, whereas $52.0 \%$ of youth who committed a sexual offence reported high levels of pre-charge social support, this was reduced to $45 \%$ post-charge. This downward shift resulted in an increase in the proportion of youth who reported moderate levels of support $(28.6 \%$ to $35 \%)$, but did not change the proportion of youth who reported low support $(12.0 \%$ to $13.0 \%)$. In contrast, the proportion of youth who committed a non-sexual offence across social support categories remained relatively stable from pre- to post-charge. Specifically, there were no substantial pre- to post-charge changes in the proportion of youth who reported high levels of support (22.2\% to $18.8 \%)$, moderate levels of support $(63.5 \%$ to $67.2 \%)$, or low levels of support $(14.3 \%$ to $14.1 \%)$.

\section{Length of Time Since First Offence}

To test whether reported changes in anxiety, depression, and self-esteem were related to the length of time spent in the criminal justice system, we calculated a "time since index offence charge" variable using the information collected on the summary sheets (described above). A sub-set of youth (16.7\%) who participated in the current 
study were referred to the recruitment clinics from Youth Mental Health Court (YMHC), a specialized diversion court for youth with diagnosed and/or suspected mental health issues (see Davis, Peterson-Badali, \& Skilling, 2016) for a program description of the Toronto YMHC). A central aim of YMHCs is to reduce the adjudication period for youth, as such the length of time since the index offence change was significantly shorter for youth referred from YMHC compared to the youth referred from standard youth criminal court, $t(77)=2.58, p=.01$. Therefore, the decision was made to remove these youth from the remaining analyses to avoid biased time estimates.

It is important to note youth referred from YMHC did not significantly differ from those referred from standard youth criminal court in regard to pre-charge anxiety, depression, or self-esteem. Meaning, the inclusion of youth from YMHC in the above analyses did not inflate pre-charge means, or bias the results. This may seem surprising given the purpose of YMHCs is to divert young people with mental health issues. However, existing evidence on YMHCs suggests the referral process is complicated and often arbitrary, resulting in few observable differences as it relates to the characteristics of the youth, including the severity of mental health needs, between those in YMHC and those in standard criminal youth court (Davis et al., 2016; Haines, Lane, McGuire, Perkins, \& Whittington, 2015).

Table 7 presents the linear regression results examining whether length of time since index offence charge predicted pre- to post-charge changes in anxiety, depression, and self-esteem. Because the variability between pre- and post-charge social support scores was very small, we excluded social support as an outcome variable in this set of analyses. Also, due to sample size restrictions, we did not report separate regression 
models for youth who committed a sexual offence $(n=19)$ and youth who committed a non-sexual offence $(n=46)$. There was no significant difference between youth who committed a sexual offence and those who committed a non-sexual offence in terms of length of time since index offence charge, $t(63)=1.47, p=0.15$. It is therefore statistically not likely the current regression analyses are biased due to mixing of offence types.

Overall, length of time since index offence charge did significantly predict pre- to post-charge anxiety, depression, and self-esteem change scores. All three outcomes were similarly associated with length of time since index offence charge, with every one month increase resulting in an approximate 0.3 to 0.4 increase in change scores. As greater change scores represent greater deterioration in outcomes from pre- to post-charge, this finding is consistent with the study hypothesis.

\section{Table 7}

Regression results of time since index offence charge predicting changes in reported anxiety, depression, and self-esteem

\begin{tabular}{|c|c|c|c|c|c|c|}
\hline & $\mathbf{N}$ & $B$ (SE) & $\beta$ & $t$-value & $95 \%$ CI & $\mathbf{R}^{2}$ \\
\hline \multicolumn{7}{|l|}{ Anxiety } \\
\hline Time since index & 65 & $0.30(0.12)$ & 0.30 & $2.48^{*}$ & $0.06,0.54$ & $0.09^{*}$ \\
\hline \multicolumn{7}{|l|}{ Depression } \\
\hline Time since index & 65 & $0.31(0.10)$ & 0.37 & $3.20^{* *}$ & $0.12,0.50$ & $0.14^{* *}$ \\
\hline \multicolumn{7}{|l|}{ Self-esteem } \\
\hline Time since index & 64 & $0.34(0.10)$ & 0.41 & $3.54^{* * *}$ & $0.15,0.53$ & $0.17^{* * *}$ \\
\hline
\end{tabular}

Notes. ${ }^{*} p<.05,{ }^{* *} p<01,{ }^{* * *} p<.001$ 
In terms of model fit, length of time since index offence accounted for the least amount of model variance when predicting reported anxiety change scores (9\%). Length of time since index offence accounted for almost twice the model variance when predicting depression and self-esteem change score (14\% and $17 \%$, respectively).

\section{Discussion}

The current study adds to the literature regarding the effects of criminal justice system involvement on youth by examining changes in mental health and social support as youth progress through the adjudication period. All a priori study hypotheses were supported: 1) significant negative changes in reported anxiety, depression, self-esteem, and perceived social support from pre- to post-charge were observed; 2) youth who committed sexual offences reported greater negative changes across all mental health variables from pre- to post-charge compared to youth who committed non-sexual offences; and, 3) length of time since the index offence significantly predicted greater negative changes in anxiety, depression, and self-esteem. Together, these findings fill a gap in the existing research landscape, which to date has primarily focused on how custodial placement affects the mental health needs of youth. By focusing on the experiences of youth during the adjudication stage of the youth criminal justice system process, the current study makes an important contribution toward better understanding how upstream criminal justice system involvement affects youth.

The observed increase in reported anxiety and depression, and the decrease in reported self-esteem, indicate the adjudication period is a vulnerable time for justiceinvolved youth as it relates to their mental health. This is an important finding as the adjudication period is a time of legal limbo where youth are dealing with the significant 
transition of entering the youth criminal justice system yet are often not eligible and/or do not have access to formal mental health supports. This is because from a policy perspective, the youth criminal justice system is structured to provide mental health support within a rehabilitation context, meaning only after the adjudication period is over. However, it is clear from the current findings that justice-involved youth require mental health support not only as part of their formal rehabilitation, but also during the court process.

It is important to emphasize the current findings present perceived changes in psychosocial functioning attributed to the adjudication period by the youth in the sample. These findings provide valuable youth-centered data regarding how youth perceive the adjudication period to have affected them over time. It is not possible to determine from the current study design whether the reported changes are specifically caused by involvement in the youth criminal justice system. In order to determine a causal relationship between the adjudication process and changes in psychosocial functioning among justice-involved youth, prospective and experimental study designs are required. What such studies could look like is discussed below (p. 91).

The issue of causality raises interesting considerations for future youth justice research in this domain regarding the nested nature of the criminal justice system. From a social ecological perspective, the youth criminal justice system is interconnected with other macro-level (e.g., education system) and meso-level systems (e.g., family system). Meaning, the disruptive force of the youth criminal justice system is rarely contained strictly within itself but cascades into other important structures within the youth's life. As such, it may not be possible to specifically isolate the singular effect (whether causal 
or not) of the youth criminal justice system from the broader disruption that can occur in conjunction with criminal justice system involvement. For example, while court appearances are an activity required by the youth criminal justice system, we cannot necessarily decipher whether it is the court appearances themselves that cause negative psychosocial effects, or if it is the collateral consequence of missing school, or the internalized stress of having caregivers take time off work, or the combination of all factors. Overall, all youth justice research must be interpreted through the lens that the youth criminal justice system is not a closed system unassociated with the broader social ecological context of justice-involved youth.

\section{Sexual Versus Non-Sexual Offence Type}

Youth who committed a sexual offence reported significantly greater perceived negative changes attributed to the adjudication process in terms of anxiety and depression compared to youth who committed a non-sexual offence. This is consistent with the original study hypothesis. These are important findings, as negative reports of mental health among justice-involved youth charged with sexual offences have been associated with negative long-term consequences. For example, using data from the Pathways to Desistance project (www.pathwaysstudy.pitt.edu), Fanniff, Schubert, Mulvey, Iselin, and Piquero (2017) found that post-release school and employment success was negatively associated with baseline mood and anxiety problems among youth who committed sexual offences. In contrast, baseline mental health problems were not associated with postrelease school and employment success among youth who committed non-sexual offences. 
One possible explanation for the observed differences based on offence type reported in the current study is that youth who committed sexual offences struggled more as they progressed through the adjudication period because of the sexual nature of their offence. Although no other studies to date have examined the differential effects associated with the adjudication period on different groups of justice-involved youth, the current findings are consistent with the general body of literature regarding the increased negative psychosocial consequences of being labelled a sexual offender for youth (e.g., Letourneau and Caldwell, 2013; Letourneau et al., 2018).

Another possible explanation for the current findings is the youth from each group differed on certain factors not accounted for in the current study. A general limitation of comparisons based on offence type is the inability to randomly assign youth to specific offence type conditions. Although several theoretically relevant factors, including age, maltreatment history, and child protection service involvement, did not differ across groups, it is not possible to rule out the influence of other group-level factors on the observed findings.

Etiological implications. Youth who committed sexual offences reported more severe negative changes in mental health problems from pre- to post charge, resulting in twice as many youth who committed a sexual offence who met the clinical cut-off for anxiety and depression at the post-charge stage compared to youth who committed a nonsexual offence. The pattern of pre- and post-charge anxiety and depression scores between youth who committed sexual offences and those who committed non-sexual offences was not as expected. Based on the generally robust finding that youth who commit sexual offences experience greater psychopathology compared to youth who 
commit non-sexual offences, we would expect to see greater anxiety and depression scores reported from the sexual offence group at both the pre- and post-charge stages. Instead, youth who committed a sexual offence reported lower anxiety and depression scores at pre-charge compared to those who committed a non-sexual offence. If this pattern does reflect offence type effects on psychopathology, it has some important etiological implications.

The observed pattern of pre-charge scores in the current study adds to the evidence that supports the assertion that differences between youth who commit sexual offences and those who commit non-sexual offences reported post-adjudication are not necessarily equivalent to differences pre-adjudication. The implication of establishing non-equivalence between pre-charge and post-charge reports is that etiological models of youth offending that include psychopathology factors, and are based on data gathered post-adjudication, may be biased due to the differential negative effects of criminal justice system involvement experienced by different groups of justice-involved youth. While many psychopathology self-report measures and structured clinical assessment guides ask about symptoms within the past 6 months (e.g., the Youth Self-Report, and Diagnostic Interview Schedule for Children), by the time most justice-involved youth participate in research (or clinical assessments coded for research) they have likely already been in the criminal justice system for at least that amount of time. In the current study, the average adjudication period was 10 months. This is consistent with national Canadian data which shows the median length of a court cases (not including time from police charge to first court appearance) for violent offences is 6-months, and for sexual offences is 9 months (Miladinovic, 2016). 
As discussed by Seto and Lalumière (2010), distinguishing the timing of psychopathology among justice-involved youth in relation to the onset of offending is critically important when trying to establish a causal etiological model. Although their discussion was referring specifically to etiological models of youth sexual offending, the premise applies to etiological models of non-sexual offending as well. For example, psychopathology is considered in several etiological models of youth sexual offending (Barbaree \& Marshall, 2008; Longo \& Prescott, 2011; Ryan, Lane, \& Leversee, 2010; Ward \& Beech, 2006) but is not widely considered a driving etiological factor for youth violent offending (Farrington, 2008; Quinsey, Skilling, Lalumière, Craig, 2004). However, the current findings suggest pre-charge anxiety and depression are more, if not equally, prevalent among youth who commit non-sexual offences. It is possible the role of psychopathology, specifically anxiety and depression, is masked by overt antisocial behaviours (Carswell, Maughan, Davis, Davenport, \& Goddard, 2004; Rawal, Romansky, Jenuwine, \& Lyons, 2004). This aligns with discussions put forth by Liebenderg and Unger (2014) who suggest depression in youth who commit violent offences is often missed because it can present as aggression and is therefore misattributed as a facet of their delinquency, as opposed to their psychopathology. It is important to note how the over-representation of youth of colour, predominantly Black youth, in the youth justice system may have biased etiological models of violent offending away from incorporating psychopathology factors. Specifically, research has shown that standardized depression measures often under-estimate the degree of depression severity for Black youth compared to White youth (e.g., Joe, Baser, Neighbors, Caldwell, \& Jackson, 2009; Taylor, Grant, Zulauf, Fowler, Meyerson, \& Irsheid, 2016). Depression also often 
manifests among Black youth as interpersonal struggles and conflict which increases the likelihood of misattribution toward aggression (Lu, Lindsey, Irsheid, \& Nebbitt, 2017).

\section{Policy Implications}

Shifting youth criminal justice system policy and practice to address the mental health needs of justice-involved youth during the adjudication period is relevant for the Canadian context for several reasons. First, the YCJA (2002) preamble directly stipulates that the youth criminal justice system process should not be harmful to the psychosocial development of youth. The current findings suggest the youth criminal justice system may not be meeting this standard. Correctional policy and practice strategies should therefore be in place to mitigate the negative psychological consequences that are empirically identified as likely associated with the adjudication process, just as strategies exist to prevent harm that has been empirically identified to be likely associated with custodial placement.

Second, the median length of the adjudication period across Canada has steadily increased over time. National statistics regarding the average length of adjudication for justice-involved youth, defined here as the period from being charged to case resolution, are not available. However, based on national youth courts data, the median length of time from first court appearance (not including bail hearings) to case resolution has significantly increased over time. In a report by the Department of Justice of Canada (2016) the median length of time to complete a youth court case increased from 75 days in the year 2000 to 120 days in the year 2014. Similar increases are observed specifically for violent offence cases, which reflects the offence types of the current sample. For example, in the year 2007 the median length for a violent court cases was 148 days 
(Thomas, 2009), compared to 170 days in the year 2015 (Miladinovic, 2016). Of most concern in light of the current findings, is the fact that sexual offence cases consistently have the longest court processing time of any youth offence category. Recent data shows the median length of time to complete a sexual assault youth court case is 258 days, and for other sexual offences (e.g., sexual interference) is 243 days.

The current findings provide two compelling pieces of evidence that support policy and practice changes to reduce court case processing times for youth. First, regression findings indicate the longer justice-involved youth are involved in the adjudication process, the greater the negative effect on their mental health. Second, while study findings show all youth experienced an increase in mental health issues from preto post-charge, youth who committed sexual offences reported the greatest negative changes. Taking these two findings together, it seems paradoxical for youth sexual offence cases to have the longest adjudication times. Ideally, there should be an overall reduction in the adjudication times for justice-involved youth, especially for those who committed a sexual offence; however, it is important to acknowledge that pragmatically this may be a lofty goal considering the existing youth criminal justice system context. Further, imposed shorter adjudication times may interfere with a youth's right to due process. As such, a more pragmatic correctional policy and practice response may be to introduce strategies to mitigate the potential harm of prolonged adjudication periods.

Third, the most recent Canadian statistics show only $56 \%$ of youth are found guilty at the end of their adjudication period (Miladinovic, 2016). This means that for approximately half of all justice-involved youth, they leave the youth criminal justice system without any formal supports after a stressful, and potentially psychologically 
harmful, adjudication process. Further, even among those sentenced, 25\% receive a community service order which typically do not include orders for psychosocial treatment programs.

Finally, it is in the best interest of the youth criminal justice system, and the youth themselves, that mental health issues associated with the adjudication process are mitigated before youth enter custodial facilities or community-based treatment programs. Doing so could alleviate some of the burden placed on correctional services by the everincreasing mental health needs of justice-involved youth (Federal Advisory Committee on Juvenile Justice, 2011; Leschied, 2011). In a recent study by McCormick and colleagues (2017), justice-involved youth on probation were more likely to have their criminogenic needs addressed by probation services if their mental health needs were also addressed. These results suggest if the mental health needs of justice-involved youth could be addressed downstream in the youth criminal justice system process, not only would this positively benefit the youth, but it may also increase the effectiveness of upstream rehabilitation efforts.

\section{Limitations and Future Directions}

A limitation of the current study is the retrospective pretest and post-test design. Youth were asked to report how they felt prior to entering the criminal justice system, which on average meant thinking back retrospectively 10 months. It is therefore possible pre-charge reports do not accurately reflect the degree of problems that were present at that time. Specifically, asking youth to retrospectively assess their pre-charge state at the same time as their current state may have introduced recall bias, and limits our ability to draw causal conclusions. However, the current study design was partially chosen because 
it has the advantage of mitigating response shift bias (Howard, 1980). Self-report measures of psychological constructs are susceptible to response shift bias, which occurs when the individual's frame of reference for a particular construct (e.g., self-esteem) changes over time, or as a result of an intervention. The rationale for implementing a retrospective pre-test and post-test in the current study was that justice-involved youth are likely to endure shifts in their internal frame of reference for multiple psychological constructs due to the experience of being in the youth criminal justice system. Further, from a developmental perspective, justice-involved youth are at a critical period of psychosocial development which suggests their internal frame of reference for psychosocial constructs is naturally changing over time. Further, while prospective pretests are often favored, several studies have demonstrated the validity of retrospective pre-tests when evaluating change among children and youth (e.g., Brook, Akin, Lloyd, Bhattarai, \& McDonald, 2016; Sibthorp, Paisley, Gookin, \& Ward, 2017).

Another advantage of the chosen retrospective pre-test and post-test design is the mitigation of statistical regression toward the mean. One possible limitation of change scores calculated using prospective pre-tests and post-tests is a tendency for individual post-test scores administered at a subsequent time point to regress toward the population mean. This potential limitation of change scores is mitigated in the current study because both pre-test and post-test scores were administered simultaneously. That being said, there are other limitations of using change scores, including possible ceiling and floor effects. Meaning, the variance of the change score is restricted due to restricted variability in the component scores. Change scores are also susceptible to low reliability (Edwards, 2001; Rogosa \& Willet, 1983). 
The chosen study design also limits the ability to determine whether pre- to postcharge changes persisted beyond the adjudication period. Although examining the persistence of mental health problems post-adjudication was not a goal of the current study, future work using a longitudinal design would allow for a better understanding of the trajectory of mental health issues through the youth criminal justice process. While several longitudinal studies have examined the long-term outcomes of youth criminal justice system involvement (e.g., Abram et al., 2015; Teplin et al., 2012), most of these studies examine outcomes from the time youth are released. Missing from the literature are longitudinal multi-wave studies where baseline is measured at first contact with the youth criminal justice system, and follow-up time points correspond to different stages in the youth criminal justice system process as opposed to arbitrary time-points. This type of study design would be advantageous for the field of youth justice research, as it would allow for conclusions to be drawn regarding critical judicial events, but also conclusions regarding the possibility of cumulative strain across social systems. As discussed, the youth criminal justice system does not operate in isolation from social ecological levels (e.g., schooling, friends, family, work).

Determining the relationship between youth criminal justice system involvement and psychosocial consequences would also benefit from experimental study designs whereby justice-involved youth are randomly assigned to diversion or regular judicial processing. This type of study design would address causality criteria such as temporal precedence and specificity (see Hill, 1965). Random assignment to system processing conditions would also rule out third-party variable explanations that were not necessarily accounted for in the current study design. 
The youth in the current study may have been predisposed to exacerbation effects associated with youth criminal justice system involvement because they were all referred for a court-ordered mental health assessment. It is therefore likely most youth in the current sample either had pre-existing mental health issues or possessed mental health risk markers. However, this does not mean the current findings cannot be generalized to other justice-involved youth. Due to the high prevalence of mental health issues and risk markers in the general population of justice-involved youth, the youth in the current sample do not necessarily represent a unique sub-population of justice-involved youth. That being said, future work should use an expanded study sample to determine whether the current study findings are replicated within a general population of youth.

It is also not possible to determine how the study findings were affected by the youth's bail conditions (e.g., not being able to leave the house to play sports). The youth who participated in the current study committed severe offences and/or had a history of repeated offences. As such, most had restrictive bail conditions. Research examining the effect of bail conditions on justice-involved youth is sparse, with most focusing on courtrelated outcomes such as court attendance (e.g., Sprott \& Myers, 2011). However, incorporating bail conditions as a variable in the analysis of pre- to post-charge adjudication outcomes may provide a more nuanced understanding of how the adjudication period affects justice-involved youth. For example, it is possible the current study missed an important interaction effect between bail condition severity and length of time since index offence. 


\section{Conclusion}

A central conclusion of the current study is that youth criminal justice system involvement is associated with greater deterioration of mental health among youth who commit sexual offences compared to those who do not. Figures 2,3 , and 4 clearly show the negative trajectory associated with youth criminal justice system involvement is much steeper for youth who commit sexual offences compared to those who do not. We know from the current findings this is not because of lengthier adjudication times. As such, the question remains: why does this effect occur?

A possible individual-level explanation may be bias among court officials. There is some research to suggest individual perceptions of sexual offending informs how court officials make decisions (e.g., Bouhours \& Daly, 2007; Nhan, Polzer, \& Ferguson, 2010; Sample \& Kadleck, 2008). Findings from the United States would also point toward the possibility that discriminatory system-level policies could account for this effect.

Although the legislative landscape in Canada does not include the same type of restrictive policies as in the United States (e.g., registration and notification laws for children and youth), youth who commit sexual offences in Canada are still eligible for some unique correctional provisions due to the sexual nature of their offence. For example, youth who commit sexual offences in Canada are eligible for restrictive bail conditions regarding access to children, which can then limit their access to parks, organized sports and activities, and sometimes their own siblings. In line with the proposed explanation above that more restrictive bail conditions may account for greater negative changes in mental health among all justice-involved youth, it is possible youth who commit sexual offences are differentially impacted the unique bail conditions imposed on them. 
Cutting across both individual- and system-level explanations is the potential role of stigma in explaining the differential effects of youth criminal justice system involvement observed in the current study. Although the stigma associated with sexual offending was discussed throughout this paper, the current study did not explicitly account for stigma in relation to the variables examined. Future work should specifically examine the relationship between stigma and mental health issues among youth who commit sexual offences. Doing so may contribute to a better understanding of the mechanism(s) behind why youth who commit sexual offences fare more poorly from a psychosocial perspective in the youth criminal justice system compared to youth who commit non-sexual offences. 


\section{Chapter Bridge: From Pre-Post Adjudication Changes in Mental Health to Internalized Offending-Based Stigma}

\section{Study 2 Summary}

Study 2 suggests justice-involved youth who progress through the youth criminal justice system experience negative consequences related to their mental health and social support networks. These findings suggest negative changes to mental health and social support is a collateral consequence of youth criminal justice system involvement. Another possible collateral consequence experienced by youth as they become involved in the youth criminal justice system is offending-based stigma. As discussed, the stigma associated with criminal justice system involvement may directly influence the magnitude of mental health-related consequences experienced by justice-involved youth. It may also be a key mediator in explaining differences observed between youth who commit sexual offences and youth who commit non-sexual offences. Study 3 directly builds on the findings of Study 2 by quantitatively examining the relationship between internalized offending-based stigma and reported anxiety, depression, and self-esteem. These findings (see pages 108-112) provide evidence internalized stigma is associated with the magnitude of mental health-related issues experienced by justice-involved youth. Study 3 also examines the construct of offending-based stigma using qualitative methods. Overall, Study 3 uses an interdisciplinary mixed-methods approach and intersectional theoretical framework to provide a comprehensive and nuanced analysis of offendingbased stigma among justice-involved youth.

Prioritizing differences in offence type. Distinguishing justice-involved youth based on offence type is relevant for Study 3 because of the intense social stigma 
associated with committing sexual offences (Burchfield \& Mingus, 2014). The broad stereotyping of individuals who commit sexual offences as predatory, dangerous, and impossible to rehabilitate, is often applied to youth and can affect how they are responded to by the youth criminal justice system (Seidler, 2010). There is also compelling empirical evidence that shows youth who commit sexual offences experience a high degree of psychological stress as they progress through the criminal justice system because of the stigma attached to committing a sexual offence (Letourneau et al., 2018). An example of this evidence are findings from an extensive qualitative study examining the effect of sexual offender status among youth in the United-States conducted by Human Rights Watch (2013). Among the 281 youth interviewed, 85\% reported negative psychological effects specifically attributed to their status as a sexual offender, including depression, anxiety, social isolation, and difficulty maintaining relationships.

Approximately $20 \%$ of the youth in the study reported attempting suicide, which they reported was because of the sexual offence label. To date, much of the research regarding the effects of being labelled a sexual offender has been conducted in the United States. While Canada does have less harsh and restrictive correctional policies and practices regarding youth who commit sexual offences, it cannot be assumed youth who commit sexual offences in Canada are not impacted by the broad social stigma associated with sexual offending generally. 


\section{Chapter 4: An Interdisciplinary Mixed-Method Analysis of Offending-Based Stigma Among Justice-Involved Youth}

The stigma faced by individuals involved in the criminal justice system (offending-based stigma) is a historically well-established social phenomenon. One of the first to describe this phenomenon was prison reformist Lewis E. Lawes, who detailed how upon discharge a prisoner is "conscious of the invisible stripes fastened upon him by tradition and prejudice" (Lawes, 1938, p. 298). Despite the persistence of offendingbased stigma through to today's society, there is a paucity of research in this area.

Offending-based stigma is a relatively new field of research with most of the work in this area published in the past 10 years - this is in contrast to other stigma-related research, including HIV/AIDS stigma, mental health stigma, and sexual minority stigma, that has been published since at least the 1970s. Most offending-based stigma research has also predominantly focused on previously incarcerated adult males (e.g., LeBel 2012; LeBel, Burnett, Maruna, \& Bushway, 2008; Moore \& Tangney 2017; Moore, Milam, Folk, \& Tangney, 2018). The prioritization of adults in this research domain has resulted in a limited understanding of how offending-based stigma is experienced among justiceinvolved youth.

Also missing from the literature is an interdisciplinary approach to the study of offending-based stigma. As described by Campbell and Deacon (2006), stigma is a phenomenon "rooted in the individual psyche, yet constantly mediated by the material, political, institutional, and symbolic context" (p. 416). Because of various epistemological and ontological boundaries, no one discipline is able to explain the individual-level experience of stigma while simultaneously explaining the socio-cultural 
and historical-political context that creates it. In response to this, the current study puts forth a mixed-method interdisciplinary analysis of offending-based stigma among justiceinvolved youth by drawing on several cross-disciplinary theories and methods.

\section{Conceptual Considerations for Stigma Research}

Stigma is typically studied within the disciplines of anthropology, criminology, psychology, and sociology. Across these disciplines, stigma is defined as existing at the interface of the individual and social dimensions. There is broad recognition that stigma, as a construct, is experienced at the individual level but is grounded in the social space (Yang et al., 2007). However, how social space is theorized and conceptualized within these disciplines differs. Establishing conceptual clarity regarding the social space, and stigma more generally, is of utmost importance because it serves to locate the research in a specific paradigm which then informs the type of research questions that can be asked, and the types of methods that can be used. A clear articulation of the conceptual framework within which findings are produced also has important implications for how these findings are interpreted and understood. The current study draws on two complementary conceptual frameworks derived from social psychology and sociology to produce an integrated understanding of offending-based stigma among justice-involved youth.

Social psychology framework of stigma. A central aim of the current study is to understand the individual-level experience of offending-based stigma among justice involved youth. To do this requires adopting the assumptions underpinning a social psychology framework. This framework locates stigma as a characteristic of the individual and conceptualizes it primarily as a psychological process. For example, 
Moore, Tangney, and Stuewig (2016) refer to the internalization of offending-based stigma as a "maladaptive cognitive pattern" (p. 221). Social psychological formulations of stigma define the social aspects of stigma as psychological variables. The social space is conceptualized as a constellation of stereotypes (defined as socially shared cognitive representations; Ottati, Bodenhausen, \& Newman, 2005) and environmental cues that are cognitively and affectively appraised by the individual (Crocker, Major, \& Steele, 1998; Major \& O’Brien, 2005).

Stigma research conducted in a social psychological framework emphasizes attributional models (e.g., Corrigan, 2000; Jones et al., 1984; Stuart, 2008) and socialcognitive models (e.g., Corrigan \& Watson, 2002) of stigma. This emphasis has led to a proliferation of studies across bodies of stigma-related research (e.g., sexual minority stigma) that has substantially advanced our understanding of how individuals construct their stigmatized social identity through cognitive, affective, and behavioral processes. An example of this research with offending-based stigma is a recent study by Moore and colleagues (2016) who used structural equation modelling to test a social-cognitive model of internalized stigma originally put forth by Corrigan, Watson, and Barr (2006) in a sample of adult male inmates. The results support the conclusion that the internalization process for offending-based stigma is analogous to that of mental health-based stigma. Specifically, believing others hold negative stereotypes about offenders leads to agreement with said stereotypes, which in turn leads to the acceptance of those stereotypes as defining features of the self.

The current study draws on a social psychology framework because it accommodates research questions pertaining to the individual-level experience of 
offending-based stigma. However, this theoretical framework is not sufficient to understand how the criminal justice system, as a macro-social structure, influences the experience of stigma among justice-involved youth. This is because social psychological formulations of stigma restrict how we conceptualize the social space to material that can be cognitively appraised, therefore they do not account for the power dynamics inherent in the social construction of identities (Parker \& Aggleton, 2003). This gap does not undermine the validity of a social psychology framework, but rather simply acknowledges an epistemological boundary of the framework that limits the type of research questions that can be posed within it.

Sociological framework of stigma. Another central aim of the current study is to understand how macro-structures, particularly the criminal justice system, influence the construction and internalization of the offender identity among justice-involved youth. This type of analysis requires the adoption of a sociological framework that conceptualizes the social space as a socio-political context that affords privilege and power to certain collections of identities (Williams \& Frederick, 2015).

Stigma research conducted within a sociological framework aims to understand how macro-structures and systems create and/or reinforce negative social constructions, specifically devalued identities. Embedded in this framework are considerations of structural power (Link \& Phelan, 2001; Parker \& Aggleton, 2003). Structural power is exerted at the macro-level of society, and refers to the way institutional policies and practices create systems (e.g., the mental health system, the criminal justice system, the education system) that privilege powerful social groups while simultaneously oppressing less powerful social groups (Holley, Stromwall, \& Bashor, 2012). These policies and 
practices are fed by social discourses that are themselves patterns of meaning expressed through language that exist at the macro-level of society and are shaped by historicalpolitical context (Singer \& Hunter, 2007; Taylor \& Ussher, 2001; Wodak \& Meyer, 2009). A salient example of structural power in relation to racial identities are the Jim Crow laws that explicitly undermined the rights of Black people in the Southern States in the areas of employment, education, and public accommodation. These laws were reinforced by, and reproduced, negative social discourses about Black people in America (e.g., Black men as lazy and stupid), and contributed to racism being further structured into our social systems (Andersen \& Collins, 2004; Pincus, 1996, 1999). Racial inequality in America (and elsewhere) persists because it is embedded in the normal operations of institutions and is therefore largely invisible to those who do not experience it (Bonilla-Silva, 1997).

The current study draws on a sociology framework of stigma because it accommodates research questions pertaining to the role of the criminal justice system in shaping the experience of offending-based stigma. This paradigm assumes that it is through the institutional power and social discourses attached to the criminal justice system that the offender identity is constructed. The criminal justice system, as a macrosocial structure, legitimates differentials in power and status between individuals based on criminal justice system involvement (Herek, 2007). Status differentials between those with an offender identity and those without are reinforced by other institutions (e.g., the media, the economic system, the social service system) and culturally-embedded social discourses about offenders (e.g., offenders are dangerous; Herek, Gillis, \& Cogan, 2009). These macro-level factors converge to maintain social inequality that serves to 
disadvantage justice-involved youth while simultaneously privileging youth not involved in the criminal justice system (Foucault, 1977; Holley et al., 2012; Young, 1990/2011).

\section{Current Study}

The central research question guiding the current study is: How is offendingbased stigma experienced among justice-involved youth? As discussed, answering this research question fulsomely requires the adoption of complementary cross-discipline frameworks of stigma, each with their own assumptions and methodological boundaries. It therefore follows that no one single method can accommodate the multiplicity of epistemological and ontological considerations needed to sufficiently address the experience of offending-based stigma, because by definition it is a psychological construct experienced at the individual level, yet shaped by macro-level factors. As such, a mixed-method approach is used to answer different components of the central research question. Doing so leverages the different epistemological and ontological positions available to draw on in quantitative and qualitative approaches, as well as the complementary strengths and non-overlapping weaknesses of each method (Johnson \& Turner, 2003).

The quantitative analyses conducted in the current study examine the degree to which offending-based stigma is internalized among the study participants. As described above, the internalization of stigma is a cognitive process whereby negative social stereotypes are integrated into the individual's sense of self. Quantifying this process with the use of a psychometric scale aligns exactly with the social psychology framework described above, and provides insight into how offending-based stigma is experienced at the psychological level among youth. 
The qualitative analyses conducted in the current study examine the meaning of the offender identity among youth and how this meaning is shaped by the criminal justice system. Undertaking a qualitative analysis that incorporates the role of social systems and structural power aligns with the assumptions of a sociology framework of stigma, and provides insight to how the experience of offender-stigma among youth is shaped by macro-level factors. Combining the representativeness and generalizability of the survey data, with the rich and detailed nature of the qualitative data yields comprehensive and nuanced findings that together provide an integrated answer to how offending-based stigma is experienced among justice-involved youth.

\section{Methods}

\section{Participants}

The full sample of 90 justice-involved youth described in Study 2 above (see pages 61-62) was used for the quantitative data analyses. A subset of interviews was purposefully selected based on the analysis anchor points described below $(N=35)$ and used for the qualitative data analysis.

Study participants were not initially recruited considering specific identity axes such as race; however, the interviews included in the current analysis were purposefully sampled from the larger pool of interviews to reflect the study's anchor points. Anchor points are the specific identity categories the researcher chooses to prioritize when conducting an intersectional analysis. Anchor points can fall on the same identity axis (e.g., Black and White both fall on a race axis), or can fall on different identity axes (e.g., Black falls on the race axis whereas woman falls on the gender axis). While most intersectional research includes anchor points along axes of race, gender, and class, less 
commonly considered identity categories can also be included so long as they are conditioned on power relations (Christensen \& Jensen, 2012; Jensen \& Elg, 2010). In the current study, offence type is included as an identity axis, with particular attention paid to sexual versus non-sexual offences. A detailed description of the anchor points incorporated in the current qualitative analysis is provided below.

\section{Measures}

Internalized Stigma Scale of Offending. Internalized stigma was measured using the Internalized Stigma Scale of Offending (ISSO; Appendix N). The ISSO is a modified version of the Internalized Stigma of Mental Illness Scale (ISMIS; Ritscher, Otilingam, \& Grajales, 2003) developed specifically for the current study with the permission and approval of the original ISMIS authors. The ISSO uses the same item roots as the original ISMIS but uses the term "offender" instead of "mental illness". An example of an ISSO item is "I am disappointed in myself for being an offender"; the original ISMIS version of this item was "I am disappointed in myself for having a mental illness".

It was necessary to use a modified scale as a measure that assesses justiceinvolved youth's experience of internalized stigma does not currently exist. Careful consideration was given to using the term "offender" in the ISSO. Although the term "justice-involved youth" is considered more appropriate to refer to youth involved in the criminal justice system, its discursive meaning is not rooted in a broad societal narrative identity. In contrast, the term "offender" has a specific discursive meaning in Canadian society that relates to a particular class of individual. This meaning is derived from the socio-historical context of the Canadian justice system (e.g., Young Offenders Act, 1985), 
and cannot be easily shifted despite movement toward less stigmatizing language. The term "offender" is therefore best suited to evaluate internalized stigma related to criminal justice system involvement, until a time when it no longer invokes the embodiment of a particular marginalized identity.

The ISSO is a 29-item measure rated on a four-point Likert scale ranging from "strongly agree" to "strongly disagree". The original ISMIS has excellent psychometric properties and is the most widely validated tool to measure internalized stigma related to mental illness (Stevelink, Wu, Voorend, \& van Brakel, 2012). It measures four facets of internalized stigma: 1) alienation (e.g., "I feel out of place in the world because I am an offender"); (2) stereotype endorsement (e.g., "Offenders are violent people”); (3) perceived discrimination (e.g., "Others think I can't achieve much in life because I am an offender"); (4) social withdrawal (e.g., "I don’t socialize as much as I used to because being an offender makes me weird"). The ISSO also includes a fifth facet that is calculated and interpreted separately, stigma resistance (e.g., "Offenders make important contributions to society"). The internalized stigma total score is calculated by averaging all answered items reflecting the alienation, stereotype endorsement, perceived discrimination, and social withdrawal facets, with higher scores representing greater internalized stigma. The stigma resistance score is calculated by reverse-coding all stigma resistance items, and averaging all answered items. Higher stigma resistance scores reflect greater ability to resist stigma.

Qualitative interview. A semi-structured interview guide (Appendix O) was used to facilitate the youth interviews. The purpose of the interview was to gather in-depth qualitative data to understand the context within which youth committed their offence(s), 
and the effects of transitioning through the youth criminal justice system. The structure of the interview guide and the wording of the interview questions were iteratively revised throughout the course of data collection. Each iteration was informed by explicit feedback from youth about the interview structure and questions, the interview answers from youth participants, youth participant reactions observed during the interview by the interviewers, and interview memo notes taken by the three interviewers (i.e., myself and the two study research assistants). Iteratively revising the interview guide in this way was beneficial as it allowed for multiple perspectives to inform the interview process, ultimately producing richer data. Modifications to the interview guide naturally stopped, with the final revision completed in November 2017. It is important to note that the core interview questions were not changed, rather the structure of the interview and specific probing questions were modified in response to the iterative revision process.

The complete interview guide had 44 primary questions covering eight domains (described below). The eight domains include: (1) perception of assessment (e.g., "Can you describe what the purpose of your assessment today was?"); (2) offence details (e.g., "What was your state of mind at the time of your offence?"); (3) family (e.g., "Can you describe what your dad is like?"); (4) relationships (e.g., "Has your relationship with your friends changed since being arrested?”); (5) mental health (e.g., "Can you describe some of the ways you cope with going through the court process?"); (6) school (e.g., "Do the people at your school know about your charges?"); (7) criminal justice system (e.g., “Since being arrested have you ever been placed in custody?"); and, (8) perceptions of the future (e.g., "How do you feel about your future?"). Other questions include icebreaker questions (e.g., "Can you please describe yourself for the tape?”) and cool-down 
questions (e.g., "If you had one piece of advice to offer someone else in your position, what would it be?").

Given the complexity of each youth's experience, and the semi-structured nature of the interview, interviewers did not ask consistent follow-up questions. The follow-up questions included in the guide (Appendix N) represent examples and prompts for the interviewers based on typical responses. Globally, all follow-up questions are informed by the following questions: (1) How has their life changed since being involved in the criminal justice system? (2) How has the system affected them? (3) Are their experiences related to race, LBGTQ, and/or Indigenous, or other specific identity? (4) Are their experiences related to the type of offence they were charged with? and, (5) Are their experiences considered traumatic?

\section{Procedure}

The quantitative data used in the current study was collected as part of the survey protocol described in Study 2 above. After the survey was answered, youth were then asked if they wanted to participate in the interview, and 100\% agreed. Interviews were voice recorded, and the average interview length was 50 minutes. Youth were compensated an additional $\$ 30$ for their participation in the interview.

\section{Results}

\section{Quantitative Analysis}

ISSO psychometric properties. As described above, two ISSO scores were calculated and interpreted separately, the internalized stigma score (ISSO total) and the stigma resistance score (ISSO-resistance). This approach is recommended, as it is generally accepted that stigma resistance reflects perceived ability to deflect stigma, as 
opposed to internalized stigma itself (Livingston, Rossiter, \& Verdun-Jones, 2011; Lysaker, Roe, \& Yanos, 2007). This approach is also empirically supported by the current data. Specifically, the ISSO-resistance score was not strongly correlated with the ISSO total score $(r=.12)$. Further, the internal consistency of the four ISSO total subscales (Cronbach's $\alpha=.84$ ) was reduced when the stigma resistance scale was included (Cronbach's $\alpha=$.76). At the item level, the internal consistency of all ISSO total items (Cronbach's $\alpha=.92$ ) was reduced when the ISSO-resistance items were included (Cronbach's $\alpha=.89$ ).

An exploratory principal component factory (EFA) analysis was conducted using the 24 modified items of the ISSO total. The results indicate the ISSO total score is best conceptualized as measuring a unidimensional construct, which is consistent with the original scale (Ritsher et al., 2003). Specifically, the first factor had an eigenvalue of 8.9, and accounted for approximately $40 \%$ of the scale variance. Further, 21 of the 24 items loaded on to the first component with factor loadings $>0.53$. Although the EFA suggests the ISSO total represents a single underlying construct, the four subscales were only moderately correlated with each other (see Table 8). As such, the results for each ISSO total subscale are presented with the caveat that they do not necessarily represent distinct constructs but rather unique facets of the overarching internalized stigma construct. 


\section{Table 8}

Correlation matrix for Internalized Stigma Scale of Offending total score subscales

\begin{tabular}{|c|c|c|c|c|c|c|}
\hline & 1 & 2 & 3 & 4 & 5 & $M(S D)$ \\
\hline 1. ISSO total & --- & $.84^{* *}$ & $.82^{*}$ & $.75^{* *}$ & $.90^{* *}$ & $2.19(0.57)$ \\
\hline 2. Alienation & & --- & $.59^{* *}$ & $.48^{* *}$ & $.65^{* *}$ & $2.40(0.73)$ \\
\hline $\begin{array}{l}\text { 3. Stereotype } \\
\text { endorsement }\end{array}$ & & & --- & $.45^{* *}$ & $.70^{* *}$ & $1.87(0.52)$ \\
\hline $\begin{array}{l}\text { 4. Discrimination } \\
\text { experience }\end{array}$ & & & & --- & $.61^{* *}$ & $2.36(0.72)$ \\
\hline 5. Social withdrawal & & & & & --- & $2.23(0.73)$ \\
\hline
\end{tabular}

Degree of internalized stigma. In the full sample, ISSO total scores ranged from 1.00 to 3.83 with a mean of $2.19(S D=0.57)$. The ISSO-resistance scores ranged from 1.00 to 4.00 with a mean of $2.07(S D=0.58)$. ISSO total and ISSO-resistance scores were then compared between youth who committed sexual offences and those who committed non-sexual offences. This decision was made because of the robust body of work indicating individuals charged with a sexual offence experience greater stigma in the criminal justice system compared to those charged with a non-sexual offence (Harris \& Socia, 2016). Table 9 presents the independent samples $t$-tests values and corresponding Cohen's $d$ effect sizes for these comparisons. A negative Cohen's $d$ means the youth who committed sexual offences reported worse outcomes compared to the youth who committed non-sexual offences. As per Cohen's (1988) suggested interpretation, an effect size of 0.30 is considered small, 0.50 is considered medium, and 0.80 is considered large. 
Overall, youth who committed a sexual offence reported significantly greater internalized stigma compared to youth who committed a non-sexual offence. Large negative effect sizes are observed across the alienation $(d=-1.04)$ and social withdrawal $(d=-0.91)$ subscales, suggesting youth who committed a sexual offence struggle with these facets of stigma the most compared to youth who committed a non-sexual offence. No significant difference was found between groups of youth for the stereotype endorsement subscale, although Cohen's $d$ suggests a medium effect ( $d=-0.45)$. No significant difference was also found between groups of youth for the discrimination experiences subscale, and a small effect size was found $(d=-0.27)$.

The mean score for stigma resistance across both groups of youth was relatively low, and no significant difference was found. This suggests regardless of offence type, youth struggle to internally deflect the stigma associated with their offending identity. However, this is only a tentative conclusion, as the results of the comparison must be interpreted with caution due to the low internal consistency of the stigma resistance subscale itself (Cronbach's $\alpha=.59$ ). Poor reliability suggests the subscale items are possibly not measuring a unified construct of stigma resistance. Poor reliability also reduces statistical power which increases the likelihood of non-significance, and underestimates the true effect size (Kline, 2016; Little, Lindenberger, \& Nesselroade, 1999). 
Table 9

Independent samples t-test comparisons of Internalized Stigma Scale for Offenders

\begin{tabular}{|c|c|c|c|c|c|c|}
\hline & & Sexual Offence & Non-Sexual Offence & & & \\
\hline ISSO Scales & $N$ & Mean (SD) & Mean (SD) & $t$-value & $95 \% \mathrm{CI}$ & Cohen's $d^{a}$ \\
\hline Total score & 90 & $2.48(0.67)$ & $2.07(0.48)$ & $2.73^{* *}$ & $0.10,0.70$ & -0.76 \\
\hline Alienation & 89 & $2.90(0.68)$ & $2.21(0.66)$ & $4.36^{* * *}$ & $0.36,1.00$ & -1.04 \\
\hline Stereotype endorsement & 90 & $2.04(0.62)$ & $1.81(0.47)$ & 1.86 & $-0.02,0.47$ & -0.45 \\
\hline Discrimination experience & 89 & $2.56(0.80)$ & $2.29(0.69)$ & 1.56 & $-0.07,0.61$ & -0.37 \\
\hline Social withdrawal & 89 & $2.68(0.71)$ & $2.06(0.67)$ & $3.78^{* * *}$ & $0.29,-0.94$ & -0.91 \\
\hline Stigma resistance & 89 & $2.18(0.48)$ & $2.03(0.62)$ & 1.11 & $-0.12,0.43$ & 0.26 \\
\hline
\end{tabular}

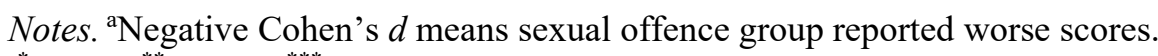

${ }^{*} p<.05,{ }^{* *} p<.01,{ }^{* * *} p<.001$ 
ISSO post-hoc testing. A possible alternative explanation for the observed significant differences between youth who committed a sexual offence and those who did not on the ISSO total score, is they are an artifact of the racial breakdown of the offence type groups. Racial differences are often observed in offending-based stigma research because greater experience being socially marginalized due to race can protect against the internalization of other stigmas (Harris, 1976; Moore et al., 2016, 2018). For example, Winnick and Bodkin (2008) found that being Black was a protective factor against offending-based stigma compared to being White. They concluded that because Black individuals have more experience deflecting racial stigma, the stigma associated with offending is less easily integrated into their self-concept. In the current study, there was a significantly lower proportion of non-White youth (youth of colour) among those who committed a sexual offence compared to those who committed a non-sexual offence (45\% versus $80 \%$ ). However, post hoc probing found no significant differences between White and non-White youth across the ISSO total score, $t(88)=0.64 p=0.52$, or stigma resistance subscale, $t(87)=0.94 p=0.35$. These post hoc findings reinforce the conclusion that the factor driving the observed significant differences between the groups of youth is their offence type.

Association of offending-based stigma with psychosocial variables. The effects associated with internalizing stigma can include increased mental health issues (Levy, Celen-Demirtas, Surguladze, \& Sweeney, 2014; Corrigan et al., 2006; Livingston \& Boyd, 2010; Moore \& Tangney, 2017). To test this association specifically in relation to offending-based stigma, youth reports of anxiety, depression, and self-esteem since they were charged with their current criminal offence were correlated with the ISSO total 
score. The stigma resistance subscale was not included in these analyses, as there is no theoretical or empirical rationale that would suggest an association between the construct of stigma resistance and mental health issues.

Table 10 presents the correlation matrix for the above associations for both youth who committed a sexual offence and youth who committed a non-sexual offence.

\section{Table 10}

\section{Correlation matrix for Internalized Stigma Scale of Offending as a function of youth}

\section{offence type}

\begin{tabular}{lccccc}
\hline & 1 & 2 & 3 & 4 & $M(S D)$ \\
\hline 1. ISSO total & --- & $.45^{*}$ & $.61^{*}$ & -.36 & $2.48(0.67)$ \\
2. Anxiety & $0.54^{* *}$ & --- & & $14.32(9.91)$ \\
3. Depression & $0.54^{* *}$ & & --- & $11.88(7.11)$ \\
4. Self-esteem & $-0.25^{*}$ & & $-1.63)$ \\
$M(S D)$ & $2.07(0.48)$ & $11.28(8.12)$ & $8.69(6.44)$ & $23.11(3.31)$ & $-10.71(4.63)$ \\
\hline
\end{tabular}

Note. Correlations and descriptive statistics for youth who committed a sexual offence $(n=24)$ are presented above the diagonal, and correlations and descriptive statistics for youth who committed a nonsexual offence $(n=64)$ are presented below the diagonal.

The pattern of reported correlation coefficients is similar across both groups of youth suggesting internalized stigma is equally associated with poor mental health and self-esteem among justice-involved youth regardless of offence type. All associations were in the expected direction. Moderate positive correlations are observed between internalized stigma, and post-charge anxiety and depression scores. Small negative correlations are also observed between internalized stigma and self-esteem. A Fisher's $r$ 
to $z$ transformation was conducted to compare the correlation coefficients from both groups (Lenhard, \& Lenhard, 2014; Lowry, 2019). The correlation coefficients did not significantly differ between youth who committed sexual offences and youth who committed non-sexual offence for anxiety $(z=-0.45, p=0.65)$, depression $(z=0.38, p=$ $0.70)$, or self-esteem $(z=0.48, p=0.63)$.

\section{Qualitative Analysis}

Theoretical approach. A limitation of most conceptual models, either within a social psychology or sociology framework, and empirical research of stigma is the emphasis on how a single identity shapes individual experience. This is an issue across bodies of stigma-related research. For example, in a systematic review of 127 published articles on internalized mental health stigma, Livingston and Boyd (2010) concluded, "despite the fact that individuals exist on multiple, intersecting axes of difference, the internalized stigma literature has primarily focused on how a single factor or status shapes people's subjective experiences" (p. 2157). To mitigate against this issue, the current qualitative analysis incorporates intersectionality as the analytical theoretical framework.

Intersectionality is appropriate for the aims of the current study because it incorporates the interplay between macro-level systems and the lived identities of individuals. It therefore aligns with the assumptions underpinning a sociological formulation of stigma. Adopting intersectionality as the analytical theoretical framework ensures the interlocking effects of multiple axes of oppression and privilege on the experience of offending-based stigma among justice-involved youth are at the forefront of the analysis (Burgess-Proctor, 2006; Cole, 2009). 
Intersectionality is historically located in the Black feminist movement (see bell hooks, 1981), and was originally coined by Crenshaw (1989) who described how the employment discrimination experienced by Black women was distinct from that experienced by Black men and White women. The central tenet of intersectionality is that socially constructed identities, primarily those reflecting race, class, and gender, are not independent constructs that exert independent influences on an individual's experience. Rather, experience is shaped by multiple identities that are inextricably intertwined, nuanced, and experienced simultaneously (Griffith, 2012; Crenshaw, 1991). Intersectionality challenges traditional single-axis thinking by explicitly incorporating the role of intersecting patterns between different structures of power. This reflects the roots of intersectionality as a concept born from oppression. For an analysis to be intersectional, it must involve an interpretation of how the historical socio-political context and social power structures mark the relationships among and between identity categories (Bowleg, 2008; Crenshaw, 2011; Cho, Crenshaw, \& McCall, 2013).

The importance of structural power in the construction of social identities, and the maintenance of social inequality, is embedded in the sociological conceptual framework of offending-based stigma and the intersectionality analytical approach adopted in the current qualitative analyses. Tying these frameworks together, the current qualitative analysis assumes: 1) the stigmatized offender identity is constructed by the criminal justice system, and maintained by other macro-level systems; and 2) the offender identity exists simultaneously with other identities, primarily those along the axes of race, class, and gender that are themselves also constructed and maintained by macro-level systems. 
Analysis anchor points. The process of choosing anchor points is necessary to keep an intersectional analysis manageable, and should be clearly rationalized to establish analytical rigor (McCall, 2005; Potter, 2013). Described below are the seven anchor points chosen for the current study: White, non-White, masculinity, working class, middle class, sexual offending, and non-sexual offending. It is important to note, the inclusion of the seven anchor points described below does not mean other identity anchor points, such as sexual orientation and disability, are not important, only that these have been deemed the most relevant to the current research question. Further, while the chosen anchor points are described here linearly this is not meant to suggest a hierarchy of oppressed identities, or that all identities function the same (Bowleg, 2008). It is important to keep in mind that race functions in a fundamentally different way than gender, which again functions differently than class. Similarly, the effects of each axis of oppression or privilege are not simply additive.

White and Non-White. The chosen analytical anchor points of White and nonWhite reflect the identity axis of race. The structural racism embedded in the criminal justice system makes race a critical axis to include when examining offending-based stigma (Bailey et al., 2017; Bonilla-Silva, 1997; Clair, \& Winter, 2016; Cunneen, 2006; Durrheim, Hook, \& Riggs, 2009; Mosher, 1998; Potter, 2013; Warde, 2013). Racial identity is central to any intersectional analysis involving the criminal justice system because White and non-White youth have differential access to power within this system. This is reflected in the countless empirical studies that demonstrate differences between Black and White youth on a range of criminal justice outcomes, including but not limited to police contact, conviction rates, sentencing decisions, and access to mental health 
treatment (Campbell et al., 2018; Claus, Vidal, \& Harmon, 2018; Hetey \& Eberhardt, 2018; Mitchell \& Caudy, 2015; Spinney et al., 2016; The Sentencing Project, 2018). Interviews from White, Black, Indigenous, and Mixed youth are included in the current analysis. Although intersectionality research has historically prioritized oppressed identities, macro-level systems and structural power condition the lives of privileged groups as well. In a recent essay by Crenshaw (2011), she describes how everyone exists within "the matrix of power" (p. 230). Ignoring privileged identities undermines the essence of intersectionality - intersectionality exists to tear down the binary of “oppressed" versus "privileged". In line with this, Verloo (2006) argues that individuals may hold dominance along one axis and subordination along another. Including privileged categories such as Whiteness in intersectional analyses ultimately produces richer knowledge about power relations (Cho et al., 2013; Choo \& Ferree, 2010; Rogers, Scott, \& Way, 2015).

Masculinity. The chosen analytical anchor point of masculinity broadly reflects the identity axis of gender. The identity axis of gender is typically conceptualized as a dimensional structure anchored by female identities on one end, and male identities on the other end. However, in the current study, the analysis is only concerned with the discourses of masculinity and how they shape the experience(s) of male youth in the criminal justice system. The current analysis examined how being a male in the criminal justice system was taken up by the youth, and how their embodiment of varying forms of masculinity informed their offending identity.

The criminal justice system reinforces hegemonic masculinity, meaning it privileges particular ways of performing the male gender that align with the idealized 
form of masculinity dictated by the dominant culture, and existing within a specific historical socio-political context (Connell, 1995; Messerschmidt, 2004; Butler, 1990; Madfis, 2014). Because of its roots in the Black feminist movement, intersectional analysis has typically prioritized the experiences of women. Critical masculinity scholars, however, argue that the male identity must be included in intersectional analyses because the types of masculinities that can be performed and embodied by men are inextricably influenced by other identity axes, primarily race and class (Connell \& Messerschmidt, 2005; Crawshaw, 2009; Hearn, 2011). Essentially, the privilege of the male gender does not guarantee social dominance in all contexts because masculinity is not constructed or embodied equally across all men's lives (Griffith, 2012; Madfis, 2014).

All youth participants whose interviews are included in the current analysis were biologically male, identified as male, and identified as heterosexual. Heterosexuality is a cornerstone of North American hegemonic masculinity, and threats against heterosexuality are usually targeted at undermining someone's masculinity (Kimmel \& Mahler, 2003). While justice-involved youth who embody traditional masculinity and heteronormativity may be privileged within the criminal justice system, their coping mechanisms may harmed by traditional scripts of stoicism. It is therefore important to understand how particular internalizations of the male identity, and its intersections with other axes, influences the experience of offending-based stigma.

Working-class and middle-class. The chosen analytical anchor points of workingclass and middle-class reflect the identity axis of race. Classism, defined as individual and institutional practices that legitimize class-based power differences which privilege the elite and middle classes while simultaneously marginalizing the poor and working 
class, is embedded in the criminal justice system (Bullock \& Limbert, 2009; Bullock, 1995). A salient example of this is the need for justice-involved youth to have access to a guardian with the financial resources to bail them out of detention. Another example is the limited access to legal representation for poor/working-class youth versus affluent youth. Within an intersectional framework, class is not strictly based on socioeconomic indicators but represents the structural system of privilege and power that exists in capitalist societies (Saegert et al., 2007). Because class is defined in terms of dominant cultural scripts, it is embodied and communicated primarily through dress and language. Similar to gender, individuals perform their class in how they dress and speak.

Interviews from youth with varying class backgrounds are included in the current analysis. Unlike the anchor points described above, class was not systematically captured by the eligibility criteria of the study or the demographic questions asked. As discussed, quantifiable socioeconomic indicators like annual family income are a limited method of capturing class, which is particularly true for youth where traditional markers of class do not necessarily apply (Bullock \& Limbert, 2009). For example, unemployment can be a marker of poverty for adults but can be a marker of wealth for youth because it reflects not needing to work because of familial resources. Similarly, there is no variance in education level among youth as it is not expected for them to have graduated high school. As such, class is analyzed by paying attention to how the youth discussed class-related stress (e.g., stress of living in a poor over-policed neighbourhood, going hungry because welfare did not afford enough food), and how they discuss performing their class (e.g., wearing specific clothing to court). 
Analytical approach. Overall, a deductive thematic analysis informed by the sixphase approach outlined by Braun and Clarke (2006) was conducted. Thematic analysis was chosen as the analytical method because it can be flexibly applied to an intersectional theoretical framework without bringing a set of its own assumptions (Braun, Clarke, \& Rance, 2014). Braun and Clarke's (2006) phases were modified to accommodate an intersectionality-informed analysis. Specifically, the six phases were divided into two distinct stages of analysis: the first stage of analysis involved identifying codes within the data, and the second stage involved explicitly contextualizing the codes within intersections of power before assembling them into latent themes.

Although multi-stage analysis is common when conducting an intersectionalityinformed qualitative analysis, there is no set method (Hankivsky \& Grace, 2014; Hunting, 2014). The second stage of analysis often varies in terms of explicitness, with some analysts doing it almost simultaneously with the coding (e.g., Galdas et al., 2012), whereas others has clearly defined stages and use templates to guide the task of incorporating the often implicit experiences of intersectionality into their interpretation (Bowleg, 2008). I chose to conduct an explicit two-stage analysis, where codes were interpreted using a modified template to ensure themes were assembled and articulated within the context of social-historical and structural inequality. This decision was made because of the existing tension of being a White, cis-gendered woman who is middleclass with no prior criminal justice system involvement, analyzing the interviews of predominantly Black cis-gendered men who are poor and involved in the criminal justice system. As such, I could not assume the role of structural power in shaping the experiences of the study participants would be obvious, or automatically at the forefront 
of the analysis. The other two interviewers and coders were also White, middle-class, cisgendered women without prior criminal justice system involvement, which further reinforced the need to make explicit the intersectional analytical framework.

The first stage of analysis included the familiarisation with the data and coding steps outlined in Braun and Clarke's guide (2006). Familiarisation with the data was achieved by reading and re-reading the interview transcripts. This process was aided by the fact that I had conducted most of the interviews included in the current analysis ( $N=$ $30 ; 85.6 \%$ ) and had taken memos about the interviews at the time they were conducted. These memos were reviewed as part of the familiarisation process. The interview data was then coded deductively, meaning the data were coded to specifically answer the central research question: what does it mean to criminally offend for justice-involved youth? The goal of a deductive analysis is to produce a detailed and nuanced account of one particular theme within the data that maps on to the chosen theoretical approach (Braun \& Clarke, 2006; Boyatzis, 1998). This is in contrast to an inductive thematic analysis where the research question evolves from the coding process, and the goal is to produce an accurate reflection of all themes identified across the entire set of data. A deductive coding approach was most appropriate because it aligns with the purpose of the current study which is to understand the latent construct of an offender identity through an intersectional lens.

The second stage of analysis included the searching for themes, reviewing themes, and defining themes steps outlined on Braun and Clarke (2006). The analytical process of assembling themes involved reinterpreting all of the codes relating to the meaning of criminally offending by asking: how do the identity axes of the youth inform how this 
meaning is constructed? This stage of analysis involved systematically reviewing each piece of coded data and contextualizing it within structures of power, regardless of whether the role of power was explicitly articulated or not. Because the interpretative work done at this stage included examining the broader assumptions, structures, and meanings underpinning the articulated experience of youth, the resulting themes are latent in nature and already theorised (Braun, Clarke, \& Rance, 2014; Taylor \& Ussher, 2001).

Devalued social identity. Across the dataset of interviews, the most common theme was that committing a criminal offence means you have a devalued social identity. There were no patterned differences identified in relation to this theme between youth who committed a sexual offence and those who committed a non-sexual offence. Regardless of offence type, youth discussed how their identity, and their personhood, was now defined in terms of negative social discourses regarding offenders broadly. This is demonstrated in the following quote where a Black gang-affiliated youth is discussing how he differs from his older brother:

My older [brother] has nothing to do with any of that. No gang or none of that criminal stuff, he's a good boy. He's in university, and he's working. He's a good boy.

The assumption underpinning the above statement is that the youth speaking is a bad person because he has criminally offended. The quote also illustrates another pattern identified across the dataset, which is that being and "doing good" is closely tied with the concept of upward mobility, which itself is underpinned by the structural system of class. How class contributes to shaping the meaning of "doing good" is discussed in the next section. 
In relation to the current theme of devalued social identity, youth clearly

articulated how committing a criminal offence means you are no longer seen as an individual with value. Instead you are constructed as a person of lower status and value, and a person who is not eligible to participate in normal society.

I feel like I've been put aside. Like I'm not somebody who can achieve anything anymore. They are telling me you aren't this, you are now this. [It's because] they don't truly know what I'm capable of...they heard everything bad about me....that's the only picture they really have of me. Just the bad. All of the good is left out.

I felt suicidal because when you're charged... and they say you're this person but they don't actually know you, like know you at all....[it] makes you feel worthless basically, and makes you feel...makes you wanna put your shoulders down, and put your head down, and just find a way to finish it, you know?

People view us like we're lesser....[especially] Black people, right now...everybody's looking at us like we're the worst ever.

A common pattern identified in the youth's narratives regarding the theme of devalued social identity was the felt permanency of their imposed lower status position. Youth discussed wanting to reclaim a valued position in society by "doing good" or "getting on the right path" but felt this was almost impossible because of how the offending identity is constructed as a permanent status in society. There was a tone of hopelessness in the discussions of youth regarding their lack of power to challenge the entrenched perception that "once an offender, always an offender".

People will always look at me different. People will always talk to me different. I feel unwelcomed, I feel like...just like a different person, you know what I mean? You feel you're not welcome, like...you fucked up once in your life and now you're always a fuck up. I don't know, I want to do good, but like...I can't.

My mom used to call me sweetie, now I don't even know what I am to her. I'm still her son, but in her mind I'm pretty sure I'm just a trouble maker...It makes me feel pretty crappy knowing that your whole family thinks you're not going to do better for your life. 
The narratives from the youth of colour, predominantly Black youth, show how discourses about race intersect with discourses of offending to uniquely position these youth as dangerous. Because of how discourses regarding race function, the offender identity applied to youth of colour takes on a particular violent twist which serves to further position as devalued members of society. The following quotes illustrate this point:

As soon as they hear that I'm [a criminal] they're not going to associate with me at all. They think I'm some fucking scary guy. It pisses me off. I know it shouldn't piss me off because it proves them right. But it just makes me mad. Like, they say I'm an angry person. Then it gets me mad, not because I am an angry person, but because of the fact they label me with that.

Everyone will look at you like "Oh, he's a criminal, he [must have] stabbed someone, be careful." It doesn't faze me because I'm not that type of person, that's what everyone just thinks. If people know that you're criminal already, they're always looking over [their shoulder] at that person. So, yes, I don't want to tell no one I'm a criminal.

If they want to see me like a risk...there's nothing I can do about it personally, because what am I supposed to do, persuade [them]? I can't persuade somebody. If they want to believe that I'm bad, and they want to keep me in the house, they have the full power to do it. I can't do anything about it because they are the judge, and then there's [just] me. I have no power over a judge.

Another common pattern identified in the narratives of youth of colour regarding how race intersects with an offender identity is being positioned as gang members. While primarily driven by racial discourses and stereotypes, class also informs social discourses about gang members. In this way, gang members are society's three-dimensional physical representation of offending, race, and class discourses.

One of the [correctional officers] called me a Blood because I was wearing red. I'm like "I wear blue too... am I a Crip now?" I mean, I told them..."What? Because I'm wearing red, I'm a gangster now?" As soon as a White kid [wears] that, it's fine...it's always the Brown and Black kids being told "Oh, you're a gangster now". 
As soon as they hear someone has a charge of anything, they're going to be scared. They think they're some gangster.

To be labelled a thug, you know what I mean...it's fucked up trying to do good and I'm just like...told I'm a real life thug. Like, what the heck?

The latter quote is also a good example of how the possibility of "doing good" feels further away for youth of colour. This point is also illustrated in the following quote from a Black youth. The implication of the underlined language is that being Black and an offender work together to create a double barrier to success.

People [already] discriminate against you just because of the way you look...if you're not a certain race they don't want you there. So...adding this is just another reason for them to not want you working for them.

Overall, when youth of colour commit criminal offences they are easily and quickly positioned as devalued members of society because of how underlying assumptions about race and class function in relation to the criminal justice system.

Restricted upward mobility. The way the offender identity is constructed for youth is heavily influenced by the concept of class. This is demonstrated in the narratives of youth regarding how their upward mobility is restricted because of the way offenders are positioned in our class system. Specifically, the existing class system reinforces the devalued social identity of those who have committed a criminal offence by excluding and/or marginalizing them within the labour structure which further positions them as low status in society. This is illustrated in the following quote from a White streetinvolved youth whose family was middle class:

Having charges...I feel like I am categorized in, um, lower class per se. And like, I could do certain jobs, but I won't be able to have the same kind of lifestyle. Or maybe I could... but like, only once [my record] is wiped years later. 
A common narrative among youth was that the possibility of their criminal record being sealed was a way out from their lower status in society. Every youth discussed how criminal records represent a barrier to having a "good life". This is an example of how social value is intertwined with our class structure, and how our class structure works with the criminal justice system to oppress individuals who have criminally offended. Youth specifically discussed how their life has more potential because they are in the youth criminal justice system as opposed to the adult system. Underpinning this narrative is the assumption that a sealed record will enable youth to move beyond their devalued status.

Thank God I am young. If I was like, over 18 I wouldn't be so lucky. [My offence] won't show in my record...so if I apply for a job it won't tell them, so it's all good.

What the lawyer told me...I'm lucky I'm not an adult because if I were, I would be registered for life.

I'm lucky I'm not an adult because if I was an adult I'd be in so much trouble. It would've been miserable. I mean, like...I feel like there's still a way out, you know?

Some youth resisted the notion that having a criminal record would prevent them from "doing good". In most cases, they drew on the experiences of people they personally knew as evidence of their potential for success despite being currently positioned as a devalued member of society. The next quote from a Mixed youth is an example of this:

I know some people who are criminals... or went through the court system. And they came out okay. Even better people.

One of my uncle's friends, he's been in the system, like, quite a few times. But he's doing a job where I don't think there any criminal checks. And he's actually,

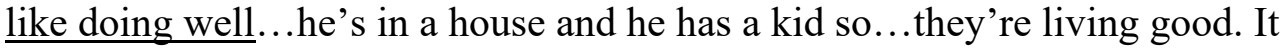


motivates me, you know? Because, like...there's someone out there who's been through what I've been through, and he's still doing [good], you know?

The above quote is to some extent a counter-narrative regarding the permanency of those with criminal records as low class; however, it is important to note how the underlined language suggests the youth understands the experience of their uncle is an exception. The above quote is also a good example of how class informs what is meant by "doing good". Specifically, doing well means fulfilling White middle-class norms. This point is also illustrated in the following quote by a youth discussing his life once his record is sealed:

I feel like I have great things I could do...have my own family, have my own house, my own dog... and I guess, live a happy life.

Restricted masculinity. The way the offender identity is constructed for youth is heavily influenced by hegemonic masculinity scripts. This point is demonstrated in the narratives of youth regarding how their masculinity is performed in the youth criminal justice system. A common theme across the interviews was that their position as an offender means restricting their masculinity to fit with hyper-masculine norms. Within the narratives of youth who came from a context where familiarity with the criminal justice system is a central part of their lived experience, predominantly Black youth from poor neighbourhoods who committed violent offences, it was clear that hegemonic masculinity scripts were implicitly reinforced as part of their normal culture. The following quotes illustrate this:

There's a code....you can't be afraid of nothing. Because by you being afraid, you're either going to be dead or be someone's bitch.

I deal with my own problems. I'm old enough. I'm not a kid [who] tells my feelings. 
Since day one of being charged, I kept everything in. Everything, like my emotions....I told nobody about out. I would go see a psychiatrist and be like "everything's good, I don't need help".

In contrast, some youth were explicitly reinforced to adopt hegemonic masculinity scripts, especially those related to emotionality. The following quotes provide examples of this:

I had a right to one free phone call, so I called my lawyer. He told me "She's gonna try to get you emotional, so try your best to hold everything in"... and I tried my best.

The officers were all big, and they looked big, and they had their guns in their pockets, and they were talking to me. And I was crying, and the officer told me "You know what? I don't feel bad for you."...I understand I did a crime but... why he gotta be like that, you know?

The latter quote is a good example of how youth who are unfamiliar with being in the criminal justice system must navigate their masculinity as it intersects with their offender identity. In this example, the youth is trying to understand why the fact that he's committed a criminal offence means he can't show emotion. The underlying message that he must adopt hegemonic masculinity because he has criminally offended is highly reinforced because he's receiving it in a hyper-masculine context with big men and their guns, both of which represent dominant subjects of masculinity in our society.

A common pattern identified in the narratives of youth of colour was how certain body features that signify greater masculinity, particularly bigger body size, shape how they are positioned in the youth criminal justice system. Many described how their offender identity was informed by the fact they looked like men as opposed to kids. The following quote illustrates this:

I've had a couple of judges ask me if I was an adult. I know some people my age that are really small, they look like kids... and they're coming out of the system, like, they get bail right away. So [my size] does makes a difference...like, when 
they see a bigger guy I guess they feel like he could handle himself in custody. But not the lesser guys, is what I think.

The underlined parts of the above quote are an example of how males who do not present as hyper-masculine are considered lesser in the youth criminal justice system. This leads to an inherent tension in the experience of being an offender for youth. Specifically, because hegemonic masculinity is heavily reinforced in the youth criminal justice system, being an offender means you represent a particular form of masculinity, one that is idealized in dominant culture. Where the tension comes in is when youth want to resist their offender identity because doing so is a threat to their masculinity. This narrative was especially common among youth of colour who came from contexts where criminal justice system involvement was a central part of their upbringing and culture. Essentially, for these youth the consequence of "doing good" means being labelled soft, which in a context dominated by hegemonic masculinity scripts means giving up power. The following quote illustrates this:

My brother is also selling [drugs].... and we have problems because he thinks he's hard. Like, he comes home with all these chains... and then he'll start flexing and call me a bitch just because he think he's tougher... because I'm trying to move away from that.

\section{Discussion}

The current study provides a comprehensive and in-depth look at how offendingbased stigma is experienced by justice-involved youth by presenting complementary quantitative and qualitative findings. This study makes an important contribution to the existing literature in the following ways. First, it is the only study to date to use a psychometric internalized stigma scale to measure offending-based stigma among youth. Second, it is the first study to examine offending-based stigma through an intersectional 
lens. Finally, it is among the first studies to integrate multiple disciplinary perspectives to the study of stigma, let alone offending-based stigma among youth.

In regard to the quantitative findings youth in the current study reported mild to moderate internalized stigma. More specifically, based on established interpretation guidelines (see Lysaker et al., 2007), youth who committed sexual offences reported moderate levels of internalized stigma, whereas youth who committed non-sexual offences reported mild levels of internalized stigma. This is inconsistent with the findings of Moore and colleagues (2016) who reported low levels of internalized stigma among their sample of adult male inmates prior to release. The means reported by Moore and colleagues (2016) and those in the current study cannot be directly compared because different scales were used; however, both scales have the same possible range, so what is considered low, mild, moderate, and high levels of internalized stigma are analogous.

A possible explanation for why justice-involved youth report greater internalized stigma compared to adults is because they are less entrenched in the criminal justice system. This reflects the paradox that seems to exist in relation to offending-based stigma for adults, where individuals from highly criminalized contexts are less likely to internalize stigma. Previous work with adults has shown that greater criminal justice system involvement protects against the internalization of offending-based stigma (Moore et al., 2018). For example, LeBel (2012) discusses how "hard core" offenders are less likely to internalize offending-based stigma because the offender label does not carry negative weight in relation to their self-concept. This effect may also help explain the differences between youth who committed sexual offences and youth who committed non-sexual offences observed in the current study. Specifically, youth who committed 
sexual offences (23.8\%) were significantly less likely to have a previous criminal history compared to youth who committed non-sexual offences $(56.4 \%), X^{2}(1, N=76)=6.46, p=$ .011. It is therefore possible youth who committed sexual offences in the current sample reported greater internalized stigma scores because they were less familiar and/or entrenched in the youth criminal justice system.

The significant differences observed between youth who committed a sexual offence and those who committed a non-sexual offence support the premise that being adjudicated for a sexual offence is associated with greater internalized stigma. A likely explanation for the greater magnitude of internalized stigma reported by youth who committed a sexual offence is the increased social stigma associated with sexually based offences. Given the robust body of work demonstrating how those convicted of a sexual offence are among the most stigmatized groups in the criminal justice system, and society broadly, it therefore follows that youth who committed a sexual offence would report greater internalized stigma compared to those who committed a non-sexual offence (e.g., Burchfiled \& Mingus, 2014; DeLuca, Vaccaro, Rudnik, Graham, Giannicchi, \& Yanos, 2017; Evans \& Cubellis, 2015; Hamilton, 2017; Higgins \& Ireland, 2009; Tewksbury, 2005, 2013; Mingus \& Burchfield, 2012; Ricciardelli \& Moir, 2013; Spencer, 2009).

Consistent with the study hypothesis, significant positive correlations between internalized stigma and mental health issues (anxiety and depression) were observed among youth who committed sexual offences. The association between internalized stigma and self-esteem was not as strong (small effect, $r<.30$ ) as expected, however it was in the predicted direction. These findings are consistent with previous work on the negative psychological effects of being labelled a sexual offender for youth (Human 
Rights Watch, 2013; Letourneau et al., 2018). Surprisingly, the magnitude of association between internalized stigma and mental health issues was greater among youth who committed a non-sexual offence.

Although the underlying social discourses driving the internal stigmatization process experienced by youth who commit sexual offences and those who commit nonsexual offences may differ, it is clear offending-based stigma is associated with mental health concerns for a range of justice-involved youth. This is consistent with previous work examining the negative psychological effects of concealing a non-visible stigma more broadly. For example, in a study conducted by Frable, Platt, and Hoey (1998), university students identified as having a non-visible stigma reported significantly higher rates of anxiety and depression, as well as lower rates of social confidence and selfesteem, compared to non-stigmatized students. The current findings are also consistent with the cognitive-affective-behavioural model developed by Pachankis (2007), which states that having a non-visible stigma contributes to increased psychological distress because of the chronic stress associated with the fear of discovery.

The two facets of internalized stigma where youth who committed sexual offences and those who committed non-sexual offences did not significantly differ was stereotype endorsement and discrimination experience. The lack of significant difference between groups in terms of discrimination experience is consistent with the qualitative findings where no patterns regarding the intersection of offence type and offender identity were identified. Conceptually, these findings (or lack thereof) align because discrimination taps into how youth experience the social world, as by definition, it is the experience of being directly marginalized/disadvantaged due to some identifiable status 
(race, gender, criminal offending status; Thornicroft, Rose, Kassam, \& Sartorius, 2007).

Based on the qualitative findings, how youth construct their offending identity in relation to the social world was not discussed as being shaped by their offence type. It therefore logically follows that when categorized by offence type, youth would not necessarily differ in how they quantify their discrimination experience. Taken together, the quantitative and qualitative findings suggest that internally, at the individual level, youth who commit sexual offences seem to differ in how they internalize offending-based stigma compared to youth who commit non-sexual offences. In contrast, at the structural and social levels, youth who commit sexual offences do not seem to differ in how they conceptualize their offending identity compared to youth who commit non-sexual offences.

Although the qualitative analysis did not yield evidence that offence type informs how youth construct their offending identity, the qualitative findings reinforce the differential effects access to power and social status have in the criminal justice system. The qualitative findings are consistent with the existing body of work examining the intersection of hegemonic masculinity and the constructed identities of justice-involved youth. For example, Messerschmidt's $(1993,1999)$ analysis of justice-involved male youth concluded that depending on their access to power, young men differentially construct their identities. Messerschmidt's (1999) discussion regarding how role expectations shape masculinity (and sexuality) for male youth depending on social position is especially relevant to the current theme of restricted masculinity. Specifically, the current findings provide evidence the youth criminal justice system reinforced hegemonic masculinity which then limits access to certain performances of masculinity, 
thereby restricting how justice-involved youth can construct their offender identity due to restrictions of their masculinity. The current findings are also consistent with a study by Abrams, Anderson-Nathe, and Aguilar (2008) who found that facets of hegemonic masculinity including competition, stoicism, sexism, and homophobia were heavily reinforced in a youth correctional facility by structural elements and interpersonal interactions between staff and the youth. Abrams and colleagues (2008) also found that youth who resisted hegemonic masculinity scripts were marginalized in the institution. Adding to the Canadian evidence base regarding the intersection of hegemonic masculinity and the youth criminal justice system, the current analysis builds on the findings of Cesaroni and Alvi (2010) who analyzed qualitative data from 350 incarcerated male youth regarding their experience in the carceral system. Cesaroni and Alvi (2010) concluded the carceral setting explicitly and implicitly reinforced scripts of hegemonic masculinity by emphasizing the importance of "not showing weakness", and "being tough and unemotional" (p. 315).

Because of the over-criminalization of poor Black communities, the cultural norms and expectations within these communities often mirror those of the criminal justice system (Hirschfield, 2008). In the current study, the expectation identified by many youth of colour was that being an offender was discussed in relation to the internalized obligation to "be hard" or "not be a bitch". This finding is consistent with Majors and Billson (1992) who found marginalized Black youth tend to display "exaggerated masculinity" because their oppressed racial and class statuses prevent the embodiment of other forms of power. The devalued positioning of being an offender in society likely represents yet another pathway to power that is cut-off from these already 
marginalized youth. In this context, you can see why the potential for their youth criminal record to be sealed is so important. It is because it represents the potential reopening of a pathway to power that would otherwise be permanently closed if they were adults. Overall, the current findings indicate that adopting hegemonic masculinity scripts represents one of the only recourse for multiply disenfranchised youth to hold on to what limited power they have access to.

The current qualitative findings are also consistent with the robust body of intersectional and critical race theory-driven work that demonstrates the stereotype of "big, Black, and dangerous" persists throughout societal systems (Dallaire, McCubbin, Morin, \& Cohen, 2000; Keating, 2007, 2016; Keating, Robertson, Francis, \& McCulloch, 2002). In line with this, multiple scholars have discussed how the positioning of people of colour, predominantly Black men, legitimizes more restrictive and punitive institutional responses across systems including the school system, the mental health system, and the criminal justice system (Cousin, 1999; Smiley \& Fakunle, 2016). As it relates specifically to the youth criminal justice system, Nowacki (2017) discusses how youth of colour elicit more punitive responses at all stages of the youth criminal justice system process, which results in the over-representation of these youth in the youth criminal justice system. This in turn reinforces the social discourse that youth of colour are dangerous and/or a threat to public safety. The current qualitative findings build on this work by demonstrating the "big, Black, and dangerous" social discourses regarding race not only inform system responses to youth of colour, but also fundamentally inform how youth of colour construct themselves in relation to their offending identity. 


\section{Conclusion}

Overall, the current study achieved its aim of empirically establishing offendingbased stigma as relevant to the experience of justice-involved youth. The quantitative findings identified the degree to which justice-involved youth internalize offending-based stigma, and highlight the need to address the potential negative effects such stigma may have on the mental health of these vulnerable youth. The qualitative analysis met the goals of an intersectional analysis, as the findings clearly demonstrate how the experience of offending-based stigma takes on nuanced meaning based on intersections of race, gender, and class. Together, these findings are a necessary step to advancing policy and advocacy efforts toward reduction the stigmatization of justice-involved youth. 


\section{Chapter 5: Discussion}

The youth criminal justice system is a complex social institution responsible for addressing the needs of vulnerable and marginalized youth. For some of these youth, the youth criminal justice system is their safety net, and represents their only opportunity to have their psychosocial needs fulsomely addressed. The current dissertation adds to the ethical and legal arguments for promoting a welfare-based youth criminal justice system by offering empirical support for the expansion of rehabilitation to include psychosocial needs, primarily mental health- and stigma-related needs. As discussed, addressing the mental health and stigma-related needs of justice-involved youth will likely have little effect on future criminal justice system involvement. In fact, mental health symptoms are inversely related to recidivism among youth (Vermeiren, Schwab-Stone, Ruchkin, De Clippele, \& Deboutter, 2002). However, not addressing these needs has the potential to disrupt the normative developmental trajectory of these youth, leading to poor individual outcomes (e.g., suicide) and system-level outcomes (e.g., greater reliance on social services). It also can interfere with responsivity to intervention, as in the case of someone who is so depressed or anxious they cannot participate in rehabilitation programs.

Overall, the findings from the studies included in this dissertation add to the evidence for a relatively robust relationship between youth criminal justice system involvement and mental health concerns. The findings from Studies 2 and 3, based on the results of approximately 100 youth from the two largest cities in Ontario, are especially salient as they provide compelling evidence to support the original premise of this dissertation: Our duty of care toward justice-involved youth must extend beyond strictly addressing criminogenic risk factors. Together, the studies presented here call for us to 
shift how we conceptualize rehabilitation from a back-end response focused on recidivism, to a broad spectrum of responses that can, and should, be initiated upstream in the youth criminal justice system process.

\section{Future Directions}

The next steps in this program of work include three lines of further study: 1) replication of current findings; 2) systems-level integration of youth criminal justice and mental health systems; and, 3) expansion to young adult population.

Replication of current findings. Although the current dissertation presents findings from a relatively large sample size, considering the population, future work should aim to replicate the existing findings with a larger, and more diverse sample of justice-involved youth. This includes an expanded focus on Indigenous youth. Indigenous youth are over-represented in the youth criminal justice system, especially in Northern Ontario and Western Canada (Corrado, Kuehn, \& Margaritescu, 2014). While addressing the needs of justice-involved Indigenous youth should be a priority, future work in this area should be Indigenous led and follow the principles of Indigenous-based research (e.g., Ontario Federation of Indigenous Friendship Centres, 2016).

It would also be ideal to replicate the current studies with a national sample of justice-involved youth. There are no national forensic assessment standards for justiceinvolved youth. This may affect the scope of psychosocial needs identified and addressed in the youth forensic assessment context, and subsequently the scope of psychosocial needs addressed by youth justice services, nationally. From a youth rights perspective, it is important to identify potential provincial and territorial discrepancies regarding the referral process, and scope, of forensic assessments to ensure all justice-involved youth 
referred for a forensic assessment in Canada have equitable access to services related to their psychosocial needs. In line with this, there are existing discrepancies across Canadian provinces and territories as it relates to youth court case processing time, with Newfoundland-Labrador, Nova Scotia, Quebec, Manitoba, and the Yukon having the longest, above national average, processing times (Miladinovic, 2016). Identifying whether the adjudication process in these provinces and territories is equally, if not more so, associated with potentially detrimental negative changes in psychosocial functioning among justice-involved youth compared to provinces and territories with shorter youth court processing times, is an important step toward establishing national guidelines and standards for addressing the needs of all Canadian youth as the progress through the youth criminal justice system.

From a generalizability standpoint, it would be beneficial to include justiceinvolved youth who have not been referred for a court-ordered forensic assessment. As discussed above, an ideal study design would be to randomly assign youth to varying system processing conditions at the time of their first court-appearance, and then track their trajectories through the youth criminal justice system while surveying them at key transition points. Examples of possible study conditions include complete systemdiversion, specialized court (e.g., youth mental health court, Aboriginal court) diversion, fast-track condition, and standard court condition. This type of randomized-control prospective longitudinal design would mitigate many of the limitations currently present in the design of Studies 2 and 3, especially retrospective recall bias and the inability to assess longer-term effects of youth criminal justice system involvement. The biggest benefit of this type of study design would be the possibility to draw causal inferences 
regarding the role of the youth criminal justice system process itself on the psychosocial functioning of justice-involved youth. While the current findings provide a strong starting point, to firmly conclude the youth criminal justice system process is detrimental to the well-being of justice-involved youth, causal mechanisms must be elucidated through more rigorous study designs.

Systems-level integration. The findings from this dissertation suggest better systems-level integration of the youth criminal justice and mental health systems is needed. Although progress has been made to narrow the gaps between the youth criminal justice and mental health systems, further work is needed to ensure more justice-involved youth have their psychosocial needs identified, and addressed, while moving through the system, especially during the adjudication period. Instead of focusing on reforming the youth criminal justice system solely from within, in an attempt to increase its capacity, it may be more feasible, and ultimately more beneficial to youth, to advance an integrated systems-of-care approach. Promoting a systems-of-care approach has the potential to result in better outcomes for justice-involved youth because no one institutional system is responsible for carrying the burden of care to address the broad psychosocial needs of these youth. Possible benefits may include more consistently applied services, and services that are more appropriately calibrated for specific needs.

A critical and necessary component of a systems-of-care approach between the youth criminal justice and mental health systems is structurally embedded pathways for cross-sector collaboration. Cross-sector collaboration must be built into the structure of these systems and not strictly reliant on communication between individual system actors. As such, a key objective of this line of future work must be to identify, and then 
evaluate, sites that have structurally embedded cross-sector collaboration. A first step would be to interview a range of professionals working in, and across, both sectors. The perspectives of frontline staff are underrepresented in youth justice research, despite being key actors in the implementation of the youth criminal justice and mental health system activities (Schwalbe, 2012). Future work would benefit from interviewing youth criminal justice and mental health professional to leverage their frontline expertise in identifying ways in which better system integration can be achieved.

This line of research should also include engaging justice-involved youth. Arguably, justice-involved youth are the most important stakeholders in the youth criminal justice system because their lives are directly affected by how the system operates. The benefit of interviewing justice-involved youth in relation to system integration is ensuring the lived experiences of these youth are included in any best practice models, or policy recommendations, stemming from this line of work. From a youth rights perspective, including youth in this line of work aligns with the UNCRC, which states youth have a right to participate in research that examines matters that concern their lives (UN General Assembly, 1989).

Young adults. In 2014, the Canadian Office of the Correctional Investigator released their annual report with a recommendation to implement a National Strategy for young adults (aged 18 to 21 years old) who have offended. In 2017, the Canadian Office of the Correctional Investigator in partnership with the Ontario Office of the Provincial Advocate for Children and Youth released a report entitled Missed Opportunities: The Experience of Young Adults Incarcerated in Federal Penitentiaries, which highlighted Correctional Service of Canada's "disappointing response" and "overall lack of progress" 
in addressing the needs of youth adults who have offended (p. 7). In 2018, the Department of Justice of Canada published the findings from the Federal Government's extensive consultations regarding transforming Canada's criminal justice system. A key recommendation stemming from these consultations was to "Improve youth justice further...by includ[ing] young adults in the youth category" (p. 13). Overall, there is a clear trajectory of increasing social and political will to apply existing youth justice principles to young adults involved in the criminal justice system, particularly those principles related to protecting their psychosocial development.

To transform the above described social and political will into actionable policy and long-lasting system reform, empirical evidence is needed to justify said responses. Future research interested in promoting welfare-based criminal justice system responses for youth cannot exclusively focus on the experiences of individuals in the youth criminal justice system. There must also be a focus on young adults in the adult criminal justice system who are equally, if not more so, vulnerable to the negative psychosocial consequences of criminal justice system involvement. 


\section{References}

Abram, K. M., Teplin, L. A., Charles, D. R., Longworth, S. L., McClelland, G. M., \& Dulcan, M. K. (2004). Posttraumatic stress disorder and trauma in youth in juvenile detention. Archives of General Psychiatry, 61(4), 403-410. doi:10.1001/archpsyc.61.4.403

Abram, K.M., Zwecker, N.A., Welty, L.J., Hershfield, J.A., Dulcan, M.K., Teplin, L.A. (2015). Comorbidity and Continuity of Psychiatric Disorders in Youth After Detention: A Prospective Longitudinal Study. JAMA Psychiatry, 72(1), 84-93. doi:10.1001/jamapsychiatry.2014.1375

Abrams, L. S., Anderson-Nathe, B., \& Aguilar, J. (2008). Constructing masculinities in juvenile corrections. Men and Masculinities, 11(1), 22-41. doi:10.1177/1097184X06291893

Achenbach, T. M. (1991). Manual for the YSR and 1991 profile. Burlington, VT: University of Vermont Department of Psychiatry.

Achenbach, T. M., \& Rescola, L. A. (2001). Manual for the ASEBA school-age forms and profiles. Burlington, VT: University of Vermont, Research Center for Children, Youth, and Families.

Achenbach, T. M., McConaughy, S. H., \& Howell, C. T. (1987). Child/Adolescent behavioral and emotional problems: Implications of cross-informant correlations for situational specificity. Psychological Bulletin, 101(2), 213-232. doi:10.1037/0033-2909.101.2.213

Aebi, M., Kuhn, C., Banaschewski, T., Grimmer, Y., Poustka, L., Steinhausen, H., \& Goodman, R. (2017). The contribution of parent and youth information to identify 
mental health disorders or problems in adolescents. Child and Adolescent Psychiatry and Mental Health, 11(1), 23. doi:10.1186/s13034-017-0160-9

Aebi, M., Linhart, S., Thun-Hohenstein, L., Bessler, C., Steinhausen, H., \& Plattner, B. (2015). Detained male adolescent offender's emotional, physical and sexual maltreatment profiles and their associations to psychiatric disorders and criminal behaviors. Journal of Abnormal Child Psychology, 43(5), 999. doi:10.1007/s10802-014-9961-y

Ahmed, S. (2013). Youth criminal justice act amendments included in the Safe Streets and Communities Act. Paper presented to the $4^{\text {th }}$ Annual Children's Academic Conference.

Aiken, L. S., \& West, S. G. (1991). Multiple regression: Testing and interpreting interactions. Newbury Park, CA: Sage.

Ambresin, A., Bennett, K., Patton, G. C., Sanci, L. A., \& Sawyer, S. M. (2013). Assessment of youth-friendly health care: A systematic review of indicators drawn from young people's perspectives. Journal of Adolescent Health, 52(6), 670-681. doi:10.1016/j.jadohealth.2012.12.014

American Psychiatric Association. (2013). Diagnostic and statistical manual of mental disorders (4th/5th ed.). Washington, DC: Author.

Andersen, M., \& Collins, P. H. (2004). Race, class, and gender (5th ed.). Belmont, CA: Wadsworth.

Andrews, D. A., Bonta, J., \& Wormith, J. S. (2011). The risk-need-responsivity (RNR) model: Does adding the good lives model contribute to effective crime 
prevention? Criminal Justice and Behavior, 38(7), 735-755.

doi: $10.1177 / 0093854811406356$

Angold, A. (2002). Diagnostic interviews with parents and children. In M. Rutters \& E.

Taylor (Eds.), Child and adolescent psychiatry: Modern approached ( $4^{\text {th }}$ ed., 32-

51). Oxford, UK: Blackwell.

Australian Institute of Health and Welfare. (2017). Young people in child protection and under youth justice supervision 2015-16. Data linkage series no. 23. Cat. no. CSI 25. Canberra: AIHW.

Bailey, Z. D., Krieger, N., Agénor, M., Graves, J., Linos, N., \& Bassett, M. T. (2017). Structural racism and health inequities in the USA: Evidence and interventions. Lancet, the, 389(10077), 1453-1463.

Bala, N. (2004). The Charter of Rights and child welfare law. In Conduct of a Child Protection File, (pp. 1-48). Toronto, ON: Law Society of Upper Canada.

Bala, N. C., Carrington, P. J., \& Roberts, J. V. (2009). Evaluating the youth criminal justice act after five years: A qualified success. Canadian Journal of Criminology and Criminal Justice, 51(2), 131-167. doi:10.3138/cjccj.51.2.131

Bala, N., Finlay, J., De Filippis, R., Hunter, K. (2015). Child welfare adolescents \& the youth justice system: Failing to respond effectively to crossover youth. Canadian Criminal Law Review, 19(1), 129-151.

Barbaree, H. E., \& Marshall, W. L. (2006). The juvenile sex offender (Second ed.) Guilford Press. 
Baskin, D., \& Sommers, I. (2014). Exposure to community violence and trajectories of violent offending. Youth Violence and Juvenile Justice, 12(4), 367-385. doi: $10.1177 / 1541204013506920$

Baskin, D., \& Sommers, I. (2015). Trajectories of exposure to community violence and mental health symptoms among serious adolescent offenders. Criminal Justice and Behavior, 42(6), 587-609. doi:10.1177/0093854814556882

Bath, K., Hawke, L. D., Skilling, T., Chaim, G., \& Henderson, J. (2019). The serviceseeking profiles of youth reporting a legal mandate or perceived coercion for substance use treatment. Addictive Behaviors, 90, 27-34.

doi:10.1016/j.addbeh.2018.10.006

Bechtel, K., Holsinger, A. M., Lowenkamp, C. T., \& Warren, M. J. (2017). A metaanalytic review of pretrial research: Risk assessment, bond type, and interventions. American Journal of Criminal Justice : AJCJ, 42(2), 443-467. doi:10.1007/s12103-016-9367-1

Becker-Haimes, E. M., Jensen-Doss, A., Birmaher, B., Kendall, P. C., \& Ginsburg, G. S. (2017). Parent-youth informant disagreement: Implications for youth anxiety treatment. Clinical Child Psychology and Psychiatry, 23(1), 42-56. doi:10.1177/1359104516689586

Becker, E. M., Jensen-Doss, A., Kendall, P. C., Birmaher, B., \& Ginsburg, G. S. (2016). All anxiety is not created equal: Correlates of Parent/Youth agreement vary across subtypes of anxiety. Journal of Psychopathology and Behavioral Assessment, 38(4), 528-537. doi:10.1007/s10862-016-9544-z 
Bickel, R., \& Campbell, A. (2002). Mental health of adolescents in custody: The use of the 'adolescent psychopathology scale' in a Tasmanian context. Australian and New Zealand Journal of Psychiatry, 36(5), 603-609.

Bird, K., Amaechi, A., Bey, N. W., \& Taliaferro, W. (2016). Realizing youth justice: Advancing education and employment through public policy and investment. Washington, DC: CLASP.

Blanchette, K., \& Brown, S. L. (2006). The assessment and treatment of women offenders: An integrative perspective. Hoboken, NJ: J. Wiley \& Sons.

Bonilla-Silva, E. (1997). Rethinking racism: Toward a structural interpretation. American Sociological Review, 62(3), 465-480. doi:10.2307/2657316

Bonnar-Kidd, K. K. (2010). Sexual offender laws and prevention of sexual violence or recidivism. American Journal of Public Health, 100(3), 412-419. doi:10.2105/AJPH.2008.153254

Bonta, J. L., \& Andrews, D. A. (2007). Risk-need-responsivity model for offender assessment and rehabilitation. Ottawa, ON: Public Safety Canada.

Bouhours, B., \& Daly, K. (2007). Youth sex offenders in court: An analysis of judicial sentencing remarks. Punishment \& Society, 9(4), 371-394. doi:10.1177/1462474507080473

Bowleg, L. (2008). When black + lesbian + woman $\neq$ black lesbian woman: The methodological challenges of qualitative and quantitative intersectionality research. Sex Roles, 59(5), 312-325. doi:10.1007/s11199-008-9400-z

Boyatzis, R. E. (1998). Transforming qualitative information: Thematic analysis and code development. Thousand Oaks, CA: Sage. 
Branson, C. E., \& Cornell, D. G. (2008). Correspondence of the Millon Adolescent Clinical Inventory (MACI) and the Personality Inventory for Youth (PIY) in juvenile offenders. Journal of Forensic Psychology Practice, 7(4), 65-100. doi:10.1300/J158v07n04_02

Braun, V., \& Clarke, V. (2006). Using thematic analysis in psychology. Qualitative Research in Psychology, 3(2), 77-101. doi:10.1191/1478088706qp063oa

Braun, V., Clarke, V. \& Rance, N. (2014) How to use thematic analysis with interview data. In Vossler, A. \& Moller, N. (Eds.), The Counselling \& Psychotherapy Research Handbook (pp. 183-197). London: Sage.

Breuk, R. E., Clauser, C. A. C., Stams, G. J. J. M., Slot, N. W., \& Doreleijers, T. A. H. (2007). The validity of questionnaire self-report of psychopathology and parentchild relationship quality in juvenile delinquents with psychiatric disorders. Journal of Adolescence, 30(5), 761-771.

British Columbia Representative for Children and Youth, \& British Columbia Office of the Provincial Health Officer. (2009). Kids, crime and care: Health and wellbeing of children in care: Youth justice experiences and outcomes. Victoria, B.C.: Representative for Children and Youth

Browne, A., \& Finkelhor, D. (1986). Impact of child sexual abuse: A review of the research. Psychological Bulletin, 99(1), 66-77. doi:10.1037/0033-2909.99.1.66

Bullock, H. E, \& Limbert, W. E. (2009). Class. In D. Fox, I. Prilleltensky, \& S. Austin (Eds.), Critical psychology: An introduction (2nd ed., pp. 214-231). Thousand Oaks, CA: SAGE Publications Ltd. 
Bullock, H. E. (1995). Class acts: Middle-class responses to the poor. In B. Lott \& D.

Maluso (Eds.), Social psychology of interpersonal discrimination (pp. 118-159). New York: Guilford Press.

Burchfield, K. B., \& Mingus, W. (2014). Sex offender reintegration: Consequences of the local neighborhood context. American Journal of Criminal Justice, 39(1), 109124. doi:10.1007/s12103-012-9195-X

Burgess-Proctor, A. (2006). Intersections of race, class, gender, and crime: Future directions for feminist criminology. Feminist Criminology, 1(1), 27-47. doi: $10.1177 / 1557085105282899$

Butler, J. (1990). Gender trouble: Feminism and the subversion of identity. London, UK: Routledge.

Campbell, C., \& Deacon, H. (2006). Unravelling the contexts of stigma: From internalisation to resistance to change. Journal of Community \& Applied Social Psychology, 16(6), 411-417. doi:10.1002/casp.901

Campbell, M. A., \& Schmidt, F. (2000). Comparison of mental health and legal factors in the disposition outcome of young offenders. Criminal Justice and Behavior, 27(6), 688-715. doi:10.1177/0093854800027006002

Campbell, N. A., Barnes, A. R., Mandalari, A., Onifade, E., Campbell, C. A., Anderson, V. R., . . Davidson, W. S. (2018). Disproportionate minority contact in the juvenile justice system: An investigation of ethnic disparity in program referral at disposition. Journal of Ethnicity in Criminal Justice, 16(2), 77-98.

doi:10.1080/15377938.2017.1347544

Canadian Bar Association. (2019). Child rights toolkit. Ottawa, ON: Author. 
Canadian Charter of Rights and Freedoms, s.7, Part I of the Constitution Act, 1982, being Schedule B to the Canada Act 1982 (UK), 1982, c11.

Canadian Institute for Health Information. (2008). Health Indicators 2008. Ottawa, ON: Author.

Canty-Mitchell, J., \& Zimet, G. D. (2000). Psychometric properties of the multidimensional scale of perceived social support in urban adolescents. American Journal of Community Psychology, 28(3), 391-400. doi:10.1023/A:1005109522457

Carswell, K., Maughan, B., Davis, H., Davenport, F., \& Goddard, N. (2004). The psychosocial needs of young offenders and adolescents from an inner city area. Journal of Adolescence, 27(4), 415-428.

doi:10.1016/j.adolescence.2004.04.003

Casey, P. M., Warren, R. K., \& Elek, J. K. (2011). Using offender risk and needs assessment information at sentencing: Guidance for courts from a national working group. New York, NY: National Center for State Courts.

Cauffman, E., \& Steinberg, L. (2012). Emerging findings from research on adolescent development and juvenile justice. Victims \& Offenders, 7(4), 428-449. doi:10.1080/15564886.2012.713901

Cauffman, E., \& Steinberg, L. (2012). Emerging findings from research on adolescent development and juvenile justice. Victims \& Offenders, 7(4), 428-449. doi:10.1080/15564886.2012.713901

Cauffman, E., Piquero, A. R., Kimonis, E., Steinberg, L., Chassin, L., \& Fagan, J. (2007). Legal, individual, and environmental predictors of court disposition in a sample of 
serious adolescent offenders. Law and Human Behavior, 31(6), 519-535. doi:10.1007/s10979-006-9076-2

Cavanagh, C., \& Cauffman, E. (2015). Viewing law and order: Mothers' and sons' justice system legitimacy attitudes and juvenile recidivism. Psychology, Public Policy, and Law, 21(4), 432-441. doi:10.1037/law0000054

Cesaroni, C., \& Peterson-Badali, M. (2005). Young offenders in custody: Risk and adjustment. Criminal Justice and Behavior, 32(3), 251-277. doi: $10.1177 / 0093854804274370$

Cesaroni, C., \& Peterson-Badali, M. (2010). Understanding the adjustment of incarcerated young offenders: A Canadian example. Youth Justice, 10(2), 107125. doi: $10.1177 / 1473225410369290$

Child, Youth and Family Services Act, S.O. 2017, c. 14, Schedule 1.

Cho, S., Crenshaw, K. W., \& McCall, L. (2013). Toward a field of intersectionality studies: Theory, applications, and praxis. Signs, 38(4), 785-810. doi: $10.1086 / 669608$

Choo, H. Y., \& Ferree, M. M. (2010). Practicing intersectionality in sociological research: A critical analysis of inclusions, interactions, and institutions in the study of inequalities. Sociological Theory, 28(2), 129-149.

Chorpita, B. F., Moffitt, C. E., \& Gray, J. (2005). Psychometric properties of the revised child anxiety and depression scale in a clinical sample. Behaviour Research and Therapy, 43(3), 309-322. doi:10.1016/j.brat.2004.02.004

Chorpita, B. F., Yim, L., Moffitt, C., Umemoto, L. A., \& Francis, S. E. (2000). Assessment of symptoms of DSM-IV anxiety and depression in children: A 
revised child anxiety and depression scale. Behaviour Research and Therapy, 38(8), 835-855. doi:10.1016/S0005-7967(99)00130-8

Christensen, A., \& Jensen, S. Q. (2012). Doing intersectional analysis: Methodological implications for qualitative research. NORA - Nordic Journal of Feminist and Gender Research, 20(2), 109-125. doi:10.1080/08038740.2012.673505

Christensen, A., Margolin, G., \& Sullaway, M. (1992). Interparental agreement on child behavior problems. Psychological Assessment, 4(4), 419-425.

Christiansen, A. K., \& Vincent, J. P. (2013). Characterization and prediction of sexual and nonsexual recidivism among adjudicated juvenile sex offenders. Behavioral Sciences \& the Law, 31(4), 506-529. doi:10.1002/bs1.2070

Clair, M., \& Winter, A. S. (2016). How judges think about racial disparities: Situational decision-making in the criminal justice system: How judges think about racial disparities. Criminology, 54(2), 332-359. doi:10.1111/1745-9125.12106

Clarke, M. C., Peterson-Badali, M., \& Skilling, T. A. (2017). The relationship between changes in dynamic risk factors and the predictive validity of risk assessments among youth offenders. Criminal Justice and Behavior, 44(10), 1340-1355. doi:10.1177/0093854817719915

Claus, R. E., Vidal, S., \& Harmon, M. (2018). Racial and ethnic disparities in the police handling of juvenile arrests. Crime \& Delinquency, 64(11), 1375-1393. doi: $10.1177 / 0011128717741615$

Cleridou, K., Patalay, P., \& Martin, P. (2017). Does parent-child agreement vary based on presenting problems? Results from a UK clinical sample. Child and Adolescent Psychiatry and Mental Health, 11(1), 22. doi:10.1186/s13034-017-0159-2 
Cohen, J. (1988). Statistical power analysis for the behavioral sciences (2nd ed.). Hillsadale, NJ: L. Erlbaum Associates.

Cohen, J., Cohen, P., West, S. G., \& Aiken, L. S. (2003). Applied multiple regression/correlation for the behavioral sciences ( ${ }^{\text {rd }}$ ed.). Mahwah, NJ: Erlbaum.

Cole, E. R. (2009). Intersectionality and research in psychology. American Psychologist, 64(3), 170-180. doi:10.1037/a0014564

Collins, W. A., \& Steinberg, L. (2006). Adolescent Development in Interpersonal Context. In N. Eisenberg, W. Damon, \& R. M. Lerner (Eds.), Handbook of child psychology: Social, emotional, and personality development (pp. 1003-1067). Hoboken, NJ, US: John Wiley \& Sons Inc.

Collishaw, S., Goodman, R., Ford, T., Rabe-Hesketh, S., \& Pickles, A. (2009). How far are associations between child, family and community factors and child psychopathology informant-specific and informant-general? Journal of Child Psychology and Psychiatry, and Allied Disciplines, 50(5), 571-580. doi:10.1111/j.1469-7610.2008.02026.x

Comack, E. (1996). Women in trouble. Halifax, Canada: Fernwood.

Comack, E. (2006). The feminist engagement with criminology. In G. Balfour \& E. Comack (Eds.), Criminalizing women (pp. 22-55). Halifax, Canada: Fernwood.

Connell, R. W. (1995) Masculinities. Cambridge, MA: Polity Press.

Connell, R. W., \& Messerschmidt, J. W. (2005). Hegemonic masculinity: Rethinking the concept. Gender and Society, 19(6), 829-859. doi:10.1177/0891243205278639 
Corrado, R. R., Freedman, L., \& Blatier, C. (2011). The over-representation of children in care in the youth criminal justice system in British Columbia: Theory and policy issues. International Journal of Child, Youth and Family Studies, 2(1/2), 99. doi:10.18357/ijcyfs21/220115429

Corrado, R. R., Kuehn, S., \& Margaritescu, I. (2014). Policy issues regarding the overrepresentation of incarcerated aboriginal young offenders in a canadian context. Youth Justice, 14(1), 40-62. doi:10.1177/1473225413520361

Corrado, R. R., McCuish, E. C., Hart, S. D., \& DeLisi, M. (2015). The role of psychopathic traits and developmental risk factors on offending trajectories from early adolescence to adulthood: A prospective study of incarcerated youth. Journal of Criminal Justice, 43(4), 357-368.

doi:10.1016/j.jcrimjus.2015.04.007

Corrigan, P. W. (2000). Mental health stigma as social attribution: Implications for research methods and attitude change. Clinical Psychology: Science and Practice, 7(1), 48-67. doi:10.1093/clipsy.7.1.48

Corrigan, P. W., \& Watson, A. C. (2002). Understanding the impact of stigma on people with mental illness. World psychiatry: official journal of the World Psychiatric Association (WPA), 1(1), 16-20.

Corrigan, P. W., Watson, A. C., \& Barr, L. (2006). The self-stigma of mental illness: Implications for self-esteem and self-efficacy. Journal of Social and Clinical Psychology, 25(8), 875-884. doi:10.1521/jscp.2006.25.8.875

Council on School Health. (2013). Out-of-school suspension and expulsion. Pediatrics, 131(3), e1000-e1007. doi:10.1542/peds.2012-3932 
Cousins, L. H. (1999). "Playing between classes": America's troubles with class, race, and gender in a black high school and community. Anthropology \& Education Quarterly, 30(3), 294-316. doi:10.1525/aeq.1999.30.3.294

Crawshaw, P. (2009). Critical perspectives on the health of men: Lessons from medical sociology. Critical Public Health, 19(3-4), 279-285.

doi:10.1080/09581590902941507

Crenshaw, K. (2011). Postcript. In H. Lutz, M. T. H. Vivar, \& L. Supik (Eds.), Framing intersectionality: Debates on a multifaceted concept in gender studies (pp. 221233). Surrey, UK: Ashgate.

Crenshaw, K. W. (1989). Demarginalizing the Intersection of Race and Sex: A Black Feminist Critique of Antidiscrimination Doctrine, Feminist Theory and Antiracist Politics. Chicago, IL: University of Chicago Legal Forum.

Crenshaw, K. W. (1991). Mapping the margins: Intersectionality, identity politics, and violence against women of color. Stanford Law Review, 43(6), 1241-1299. doi: $10.2307 / 1229039$

Crocker, J., Major, B., \& Steele, C. (1998). Social stigma In S. Fiske, D. Gilbert, \& G. Lindzey (Eds.), Handbook of social psychology (pp. 504-553). Boston, MA: McGraw-Hill. doi:10.1007/s10612-013-9203-6

Cunneen, C. (2006). Racism, discrimination and the over-representation of indigenous people in the criminal justice system: Some conceptual and explanatory issues. Current Issues in Criminal Justice, 17(3), 329-346. doi:10.1080/10345329.2006.12036363 
Dallaire, B., McCubbin, M., Morin, P., \& Cohen, D. (2000). Civil commitment due to mental illness and dangerousness: The union of law and psychiatry within a treatment-control system. Sociology of Health \& Illness, 22(5), 679-699. doi:10.1111/1467-9566.00226

Davis, K. M., Peterson-Badali, M., \& Skilling, T. A. (2016). A theoretical evaluation of a youth mental health court program model. International Journal of Law and Psychiatry, 45, 17-24. doi:10.1016/j.ijlp.2016.02.003

Davis, K. M., Peterson-Badali, M., Weagant, B., \& Skilling, T. A. (2015). A process evaluation of Toronto's first youth mental health court. Canadian Journal of Criminology and Criminal Justice, 57(2), 159-159. doi:10.3138/cjccj.2014.E10

Day, D., \& Wiesner, M. (2019). Criminal trajectories: A developmental perspective. New York, NY: NYU Press

De Los Reyes, A. (2013). Strategic objectives for improving understanding of informant discrepancies in developmental psychopathology research. Development and Psychopathology, 25(3), 669. doi:10.1017/S0954579413000096

De Los Reyes, A., \& Kazdin, A. E. (2005). Informant discrepancies in the assessment of childhood psychopathology: A critical review, theoretical framework, and recommendations for further study. Psychological Bulletin, 131(4), 483-509. doi: $10.1037 / 0033-2909.131 .4 .483$

De Los Reyes, A., \& Kazdin, A. E. (2006). Informant discrepancies in assessing child dysfunction relate to dysfunction within mother-child interactions. Journal of Child and Family Studies, 15(5), 643-661. doi:10.1007/s10826-006-9031-3 
De Los Reyes, A., \& Kazdin, A. E. (2008). When the evidence says, "yes, no, and maybe so": Attending to and interpreting inconsistent findings among evidence-based interventions. Current Directions in Psychological Science, 17(1), 47-51. doi:10.1111/j.1467-8721.2008.00546.x

De Los Reyes, A., Augenstein, T. M., Wang, M., Thomas, S. A., Drabick, D. A. G., Burgers, D. E., \& Rabinowitz, J. (2015). The validity of the multi-informant approach to assessing child and adolescent mental health. Psychological Bulletin, 141(4), 858-900. doi:10.1037/a0038498

De Los Reyes, A., Kundey, S. M. A., \& Wang, M. (2011). The end of the primary outcome measure: A research agenda for constructing its replacement. Clinical Psychology Review, 31(5), 829-838. doi:10.1016/j.cpr.2011.03.011

De Los Reyes, A., Ohannessian, C. M., \& Laird, R. D. (2016). Developmental changes in discrepancies between adolescents' and their mothers' views of family communication. Journal of Child and Family Studies, 25(3), 790-797. doi:10.1007/s10826-015-0275-7

De Los Reyes, A., Salas, S., Menzer, M. M., \& Daruwala, S. E. (2013). Criterion validity of interpreting scores from multi-informant statistical interactions as measures of informant discrepancies in psychological assessments of children and adolescents. Psychological Assessment, 25(2), 509-519. doi:10.1037/a003208

De Los Reyes, A., Youngstrom, E. A., Pabón, S. C., Youngstrom, J. K., Feeny, N. C., \& Findling, R. L. (2011). Internal consistency and associated characteristics of informant discrepancies in clinic referred youths age 11 to 17 years. Journal of 
Clinical Child \& Adolescent Psychology, 40(1), 36-53.

doi:10.1080/15374416.2011.533402

DeLuca, J. S., Vaccaro, J., Rudnik, A., Graham, N., Giannicchi, A., \& Yanos, P. T. (2018). Sociodemographic predictors of sex offender stigma: How politics impact attitudes, social distance, and perceptions of sex offender recidivism. International Journal of Offender Therapy and Comparative Criminology, 62(10), 2879-2896. doi:10.1177/0306624X17723639

Department of Justice Canada. (2016). Youth criminal justice in Canada: A compendium of statistics. Ottawa, ON: Author.

Department of Justice Canada. (2018). Transforming Canada's criminal justice system: A report on provincial and territorial stakeholder consultations. Ottawa, ON: Author.

Desmarais, S. L., \& Singh, J. P. (2013). Risk assessment instruments validated and implemented in correctional settings in the United States. New York, NY: Council of State Governments Justice Center.

Development Services Group, Inc. (2017). Intersection between mental health and the juvenile justice system: Literature review. Washington, D.C.: Office of Juvenile Justice and Delinquency Prevention.

Dirks, M. A., De Los Reyes, A., Briggs-Gowan, M., Cella, D., \& Wakschlag, L. S. (2012). Annual research review: Embracing not erasing contextual variability in children's behavior--theory and utility in the selection and use of methods and informants in developmental psychopathology. Journal of Child Psychology and Psychiatry, and Allied Disciplines, 53(5), 558. 
Dmitrieva, J., Monahan, K. C., Cauffman, E., \& Steinberg, L. (2012). Arrested development: The effects of incarceration on the development of psychosocial maturity. Development and Psychopathology, 24(3), 1073-1090. doi:10.1017/S0954579412000545

Domburgh, L., Loeber, R., Bezemer, P. D., Stallings, R., \& Stouthamer-Loeber, M. (2009). Childhood predictors of desistance and level of persistence in offending in early onset offenders. Journal of Abnormal Child Psychology, 37(7), 967-980. doi:10.1007/s10802-009-9329-X

Domburgh, L., Vermeiren, R. R. J. M., Blokland, A. A. J., \& Doreleijers, T. A. H. (2009). Delinquent development in dutch childhood arrestees: Developmental trajectories, risk factors and co-morbidity with adverse outcomes during adolescence. Journal of Abnormal Child Psychology, 37(1), 93-105. doi:10.1007/s 10802-008-9260-6

Donnellan, B. M., Kenny, D. A., Trzesniewski, K. H., Lucas, R. E., \& Conger, R. D. (2012). Using trait-state models to evaluate the longitudinal consistency of global self-esteem from adolescence to adulthood. Journal of Research in Personality, 46(6), 634-645. doi:10.1016/j.jrp.2012.07.005

Donnellan, M. B., Trzesniewski, K. H., Robins, R. W., Moffitt, T. E., \& Caspi, A. (2005). Low self-esteem is related to aggression, antisocial behavior, and delinquency. Psychological Science, 16(4), 328-335. doi:10.1111/j.0956-7976.2005.01535.x Doob, A. N., \& Gartner, R. (2013). Some recent research on sex offender and society's responses to them. Criminological Highlights, (9), 1-28. 
Drerup, L. C., Croysdale, A., \& Hoffmann, N. G. (2008). Patterns of behavioral health conditions among adolescents in a juvenile justice system. Professional Psychology: Research and Practice, 39(2), 122-128.

Duhig, A. M., Renk, K., Epstein, M. K., \& Phares, V. (2000). Interparental agreement on internalizing, externalizing, and total behavior problems: A Metaanalysis. Clinical Psychology: Science and Practice, 7(4), 435-453. doi:10.1093/clipsy.7.4.435

Durrheim, K., Hook, D., \& Riggs, D. W. (2009). Race and racism. In D. Fox, I. Prilleltensky, \& S. Austin (Eds.), Critical psychology: An introduction (2nd ed., pp. 197-214). Thousand Oaks, CA: SAGE Publications Ltd.

Ebesutani, C., Reise, S. P., Chorpita, B. F., Ale, C., Regan, J., Young, J., . . Weisz, J. R. (2012). The revised child anxiety and depression scale-short version: Scale reduction via exploratory bifactor modeling of the broad anxiety factor. Psychological Assessment, 24(4), 833. doi:10.1037/a0027283

Edwards, J. R. (1994). The study of congruence in organizational behavior research: Critique and a proposed alternative. Organizational Behavior and Human Decision Processes, 58(1), 51-100. doi:10.1006/obhd.1994.1029

Ehrlich, K. B., Cassidy, J., \& Dykas, M. J. (2011). Reporter discrepancies among parents, adolescents, and peers: Adolescent attachment and informant depressive symptoms as explanatory factors. Child Development, 82(3), 999-1012. doi:10.1111/j.1467-8624.2010.01530.x 
Elliott, D. (2015). Reimagining youth justice in Ontario: How we transformed a custodyfocused system into a spectrum of services that meet diverse youth needs. Paper presented at North American Council for Juvenile Justice, Toronto, ON.

Evans, D. N., \& Cubellis, M. A. (2015). Coping with stigma: How registered sex offenders manage their public identities. American Journal of Criminal Justice, 40(3), 593-619. doi:10.1007/s12103-014-9277-z

Fairclough, N. (2013). Critical discourse analysis and critical policy studies. Critical Policy Studies, 7(2), 177-197. doi:10.1080/19460171.2013.798239

Fanniff, A. M., Schubert, C. A., Mulvey, E. P., Iselin, A. R., \& Piquero, A. R. (2017). Risk and outcomes: Are adolescents charged with sex offenses different from other adolescent offenders? Journal of Youth and Adolescence, 46(7), 1394-1423. doi:10.1007/s10964-016-0536-9

Farrington, D. P. (Ed.). (2008). Integrated developmental \& life-course theories of offending. New Brunswick, NJ: Transaction Publishers.

Faul, F., Erdfelder, E., Buchner, A., \& Lang, A. (2009). Statistical power analyses using GPower 3.1: Tests for correlation and regression analyses. Behavior Research Methods, 41(4), 1149-1160. doi:10.3758/BRM.41.4.1149

Faul, F., Erdfelder, E., Lang, A., \& Buchner, A. (2007). GPower 3: A flexible statistical power analysis program for the social, behavioral, and biomedical sciences. Behavior Research Methods, 39(2), 175-191. doi:10.3758/BF03193146

Fazel, S., Doll, H., \& Långström, N. (2008). Mental disorders among adolescents in juvenile detention and correctional facilities: A systematic review and metaregression analysis of 25 surveys. Journal of the American Academy of Child 
and Adolescent Psychiatry, 47(9), 1010-1019.

doi:10.1097/CHI.0b013e31817eecf3

Federal Advisory Committee on Juvenile Justice. (2011). Federal Advisory Committee on Juvenile Justice Annual Report 2011. Washington, DC: Department of Justice Office of Juvenile Justice and Delinquency Prevention.

Fefer, S. A., Ogg, J. A., \& Dedrick, R. F. (2015). Use of polynomial regression to investigate biased self-perceptions and ADHD symptoms in young adolescents. Journal of Attention Disorders, 1-10.

doi: $10.1177 / 1087054715573993$

Field, A. P. (2018). Discovering statistics using IBM SPSS statistics (5th, North American ed.). Thousand Oaks, California: Sage Publications Inc.

Finlay, J. (2003) Crossover kids: Care to custody. Toronto, ON: Office of Child and Family Service Advocacy (OCFSA).

Fitzgibbon, D. W. (2008). deconstructing probation: Risk and developments in practice. Journal of Social Work Practice, 22(1), 85-101. doi:10.1080/02650530701872405

Fjermestad, K. W., Nilsen, W., Johannessen, T. D., \& Karevold, E. B. (2017). Mothers' and fathers' internalizing symptoms influence parental ratings of adolescent anxiety symptoms. Journal of Family Psychology, 31(7), 939-944. doi:10.1037/fam0000322

Forrest, C. B., Tambor, E., Riley, A. W., Ensminger, M. E., \& Starfield, B. (2000). The health profile of incarcerated male youths. Pediatrics, 105(1), 286. 
Fortin, D. (2004). A correctional programming strategy for women. Forum on Corrections Research. Ottawa: Correctional Service of Canada.

Foster, E. M., Qaseem, A., \& Connor, T. (2004). Can better mental health services reduce the risk of juvenile justice system involvement? American Journal of Public Health, 94(5), 859-865. doi:10.2105/AJPH.94.5.859

Foucault, M. (1977). Discipline and punish. New York: Pantheon.

Fowler, P. J., Tompsett, C. J., Braciszewski, J. M., Jacques-Tiura, A. J., \& Baltes, B. B. (2009). Community violence: A meta-analysis on the effect of exposure and mental health outcomes of children and adolescents. Development and Psychopathology, 21(1), 227-259. doi:10.1017/S0954579409000145

Fox, B. H., Perez, N., Cass, E., Baglivio, M. T., \& Epps, N. (2015). Trauma changes everything: Examining the relationship between adverse childhood experiences and serious, violent and chronic juvenile offenders. Child Abuse \& Neglect, 46, 163-173. doi:10.1016/j.chiabu.2015.01.011

Frable, D. E. S., Platt, L., \& Hoey, S. (1998). Concealable stigmas and positive selfperceptions: Feeling better around similar others. Journal of Personality and Social Psychology, 74(4), 909-922. doi:10.1037/0022-3514.74.4.909

Gaes, G. G., \& Bales, W. D. (2011). Deconstructing the risk principle. Criminology \& Public Policy, 10(4), 979-985. doi:10.1111/j.1745-9133.2011.00777.x

Galdas, P. M., Oliffe, J. L., Wong, S. T., Ratner, P. A., Johnson, J. L., \& Kelly, M. T. (2012). Canadian Punjabi Sikh men's experiences of lifestyle changes following myocardial infarction: Cultural connections. Ethnicity \& Health, 17(3), 253-266. doi:10.1080/13557858.2011.610440 
Geer, P. (2008). Justice served? The high cost of juvenile sex offender registration. Developments in Mental Health Law, 27(2), 33.

Gellers, J. C. (2015). GREENING CRITICAL DISCOURSE ANALYSIS: Applications to the study of environmental law. Critical Discourse Studies, 12(4), 482-493. doi:10.1080/17405904.2015.1023326

Girard, L., \& Wormith, J. S. (2004). The predictive validity of the level of service inventory-ontario revision on general and violent recidivism among various offender groups. Criminal Justice and Behavior, 31(2), 150-181. doi:10.1177/0093854803261335

Gray-Little, B., Williams, V. S. L., \& Hancock, T. D. (1997). An item response theory analysis of the Rosenberg self-esteem scale. Personality and Social Psychology Bulletin, 23(5), 443-451. doi:10.1177/0146167297235001

Greve, W., \& Enzmann, D. (2003). Self-esteem maintenance among incarcerated young males: Stabilisation through accommodative processes. International Journal of Behavioral Development, 27(1), 12-20. doi:10.1080/01650250143000562

Greve, W., Enzmann, D., \& Hosser, D. (2001). The stabilization of self-esteem among incarcerated adolescents: Accommodative and immunizing processes. International Journal of Offender Therapy and Comparative Criminology, 45(6), 749-768. doi:10.1177/0306624X01456008

Griffith, D. M. (2012). An intersectional approach to men's health. Journal of Men's Health, 9(2), 106-112. doi:10.1016/j.jomh.2012.03.003 
Grills, A. E., \& Ollendick, T. H. (2003). Multiple informant agreement and the anxiety disorders interview schedule for parents and children. Journal of the American Academy of Child \& Adolescent Psychiatry, 42(1), 30-40.

Grisso, T. (2004). Double jeopardy: Adolescent offenders with mental disorders. Chicago: University of Chicago Press.

Grisso, T. (2008). Adolescent offenders with mental disorders. The Future of Children, 18(2), 143-164. doi:10.1353/foc.0.0016

Haines, A., Lane, S., McGuire, J., Perkins, E., \& Whittington, R. (2015). Offending outcomes of a mental health youth diversion pilot scheme in england: Youth diversion and re-offending. Criminal Behaviour and Mental Health, 25(2), 126140. doi:10.1002/cbm. 1916

Hamilton, E. (2017). Identity concerns among sexual offenders: The narrative call. Practice Innovations, 2(1), 13-20. doi:10.1037/pri0000039

Hamilton, M. (2015). Risk-needs assessment: Constitutional and ethical challenges. American Criminal Law Review, 52(2), 261.

Handwerk, M. L., Larzelere, R. E., Soper, S. H., \& Friman, P. C. (1999). Parent and child discrepancies in reporting severity of problem behaviors in three out-of-home settings. Psychological Assessment, 11(1), 14-23. doi:10.1037/1040-3590.11.1.14

Hankivsy, O. \& Grace, D. (2015). Understanding and emphasizing difference and intersectionality in mixed and multimethods research. In S. N. Hesse-Biber \& R. B. Johnson (Eds), The Oxford Handbook of Mixed and Multimethods Research (pp.110-127). Oxford, UK: Oxford University Press. 
Hannah-Moffat, K. (2004). Losing ground: Gendered knowledges, parole risk, and responsibility. Social Politics: International Studies in Gender, State and Society, 11(3), 363-385. doi:10.1093/sp/jxh041

Hannah-Moffat, K. (2013). Actuarial sentencing: An 'unsettled' proposition. Justice Quarterly, 30(2), 270-296. doi:10.1080/07418825.2012.682603

Haqanee, Z., Peterson-Badali, M., \& Skilling, T. (2015). Making 'what works' work: Examining probation officers' experiences addressing the criminogenic needs of juvenile offenders. Journal of Offender Rehabilitation, 54(1), 37-59. doi:10.1080/10509674.2014.980485

Harris, A. J., \& Socia, K. M. (2016). What's in a name? Evaluating the effects of the "Sex offender" label on public opinions and beliefs. Sexual Abuse: A Journal of Research and Treatment, 28(7), 660-678. doi:10.1177/1079063214564391

Harris, A. J., Walfield, S. M., Shields, R. T., \& Letourneau, E. J. (2016). Collateral consequences of juvenile sex offender registration and notification: Results from a survey of treatment providers. Sexual Abuse: A Journal of Research and Treatment, 28(8), 770-790. doi:10.1177/1079063215574004

Harris, A. R. (1976). Race, commitment to deviance, and spoiled identity. American Sociological Review, 41(5), 432.

Hart, E. L., Lahey, B. B., Loeber, R., \& Hanson, K. S. (1994). Criterion validity of informants in the diagnosis of disruptive behavior disorders in children: A preliminary study. Journal of Consulting and Clinical Psychology, 62(2), 410414. doi:10.1037/0022-006X.62.2.410 
Hawkins, J. D., Herrenkohl, T. I., Farrington, D. P., Brewerm D., Catalano, R. F., Herachi, T. W., \& Cothern, L. (2000). Predictors of youth violence. Juvenile Justice Bulletin. Washington, DC: Department of Justice Office of Juvenile Justice and Delinquency Prevention.

Hearn, J. (2011) Neglected intersectionality in studying men: Age(ing), virtuality, transnationality. In H. Lutz, M. Vivar \& L. Supik (Eds) Framing Intersectionality: Debates on a Multi-faceted Concept in Gender Studies (pp. 89104). Farnham, UK: Ashgate.

Hecker, T., \& Steinberg, L. (2002). Psychological evaluation at juvenile court disposition. Professional Psychology: Research and Practice, 33(3), 300-306. doi:10.1037//0735-7028.33.3.300

Heitzeg, N. A. (2016). The school-to-prison pipeline: Education, discipline, and racialized double standards. Santa Barbara, CA: ABC-CLIO, LLC.

Herek, G. M. (2007). Confronting sexual stigma and prejudice: Theory and practice. Journal of Social Issues, 63(4), 905-925.

Herek, G. M., Gillis, J. R., \& Cogan, J. C. (2009). Internalized stigma among sexual minority adults: Insights from a social psychological perspective. Journal of Counseling Psychology, 56(1), 32-43. doi:10.1037/a0014672

Herjanic, B., \& Reich, W. (1997). Development of a structured psychiatric interview for children: Agreement between child and parent on individual symptoms. Journal of Abnormal Child Psychology, 25(1), 21-31. doi:10.1023/A:1025703323438

Hetey, R. C., \& Eberhardt, J. L. (2018). The numbers don't speak for themselves: Racial disparities and the persistence of inequality in the criminal justice system. Current 
Directions in Psychological Science, 27(3), 183-187.

doi:10.1177/0963721418763931

Higgins, C., \& Ireland, C. (2009). Attitudes towards male and female sex offenders: A comparison of forensic staff, prison officers and the general public in Northern Ireland. The British Journal of Forensic Practice, 11(1), 14-19.

doi:10.1108/14636646200900004

Hirschfield, P. (2009). Another way out: The impact of juvenile arrests on high school dropout. Sociology of Education, 82(4), 368-393.

doi:10.1177/003804070908200404

Hirschfield, P. J. (2018). Schools and crime. Annual Review of Criminology, 1(1), 149169. doi:10.1146/annurev-criminol-032317-092358

Hoge, R. D., \& Andrews, D. A. (2010). Evaluation for risk of violence in juvenile. New York, NY: Oxford University Press.

Holley, L. C., Stromwall, L. K., \& Bashor, K. H. (2012). Reconceptualizing stigma: Toward a critical anti-oppressive paradigm. Stigma Research and Action, 2(2), 51-61. doi:10.5463/SRA.v1i1.9

Holloway, E. D., Cruise, K. R., Morin, S. L., Kaufman, H., \& Steele, R. D. (2018). Juvenile probation officers' evaluation of traumatic event exposures and traumatic stress symptoms as responsivity factors in risk assessment and case planning. Law and Human Behavior, 42(4), 369-384. doi:10.1037/1hb0000283

hooks, b. (1989). Talking back: Thinking feminist, thinking black. Boston, MA: South End Press. 
Hornor, G. (2010). Child sexual abuse: Consequences and implications.Journal of Pediatric Health Care, 24(6), 358-364. doi:10.1016/j.pedhc.2009.07.003

How, A. (2003). Critical theory. New York, NY: Palgrave MacMillan.

Hubbard, D. J. (2007). Getting the most out of correctional treatment: Testing the responsivity principle on male and female offenders. Federal Probation, 71(1), 2.

Human Rights Watch. (2013). Raised on the registry: The irreparable harm of placing children on sex offender registries in the US. New York, NY: Author.

Hunsley, J., \& Mash, E. J. (2007). Evidence-based assessment. Annual Review of Clinical Psychology, 3(1), 29-51. doi:10.1146/annurev.clinpsy.3.022806.091419

Hunting, G. (2014). Intersectionality-informed qualitative research: A primer.

Vancouver, BC: The Institute for Intersectionality Research \& Policy, SFU.

Imbach, D., Aebi, M., Metzke, C. W., Bessler, C., \& Steinhausen, H. C. (2013).

Internalizing and externalizing problems, depression, and self-esteem in nondetained male juvenile offenders. Child and Adolescent Psychiatry and Mental Health, 7(1), 7-7. doi:10.1186/1753-2000-7-7

International Society of Psychiatric-Mental Health Nurses. 2008. Meeting the Mental Health Needs of Youth in Juvenile Justice. Madison, WI: Author.

Jensen, P. S., Rubio-Stipec, M., Canino, G., Bird, H. R., Dulcan, M. K., Schwab-Stone, M. E., \& Lahey, B. B. (1999). Parent and child contributions to diagnosis of mental disorder: Are both informants always necessary? Journal of the American Academy of Child \& Adolescent Psychiatry, 38(12), 1569-1579.

doi:10.1097/00004583-199912000-00019 
Jensen, S. Q., \& Elg, C. (2010) Intersectionality as embodiment. Kvinder, Køn og Forskning, 19(3), 30-40.

Joe, S., Baser, R. S., Neighbors, H. W., Caldwell, C. H., \& Jackson, J. S. (2009). 12month and lifetime prevalence of suicide attempts among black adolescents in the national survey of American life. Journal of the American Academy of Child \& Adolescent Psychiatry, 48(3), 271-282. doi:10.1097/CHI.0b013e318195bccf

Johnson, B., \& Turner, L. A. (2003). Data collection strategies in mixed methods research. In A. Tashakkori \& C. Teddlie (Eds.), Handbook of mixed methods in social and behavioral research (pp. 297-319). Thousand Oaks, CA: Sage.

Jones, E. E., Farina, A., Hastorf, A. H., Markus, H., Miller, D. T., \& Scott, R. A. (1984). Social stigma: The psychology of marked relationships. New York: Freeman.

Jones, N. J., Brown, S. L., Robinson, D., \& Frey, D. (2015). Incorporating strengths into quantitative assessments of criminal risk for adult offenders: The service planning instrument. Criminal Justice and Behavior, 42(3), 321-338. doi: $10.1177 / 0093854814547041$

Jones, N. J., Brown, S. L., Robinson, D., \& Frey, D. (2016). Validity of the youth assessment and screening instrument: A juvenile justice tool incorporating risks, needs, and strengths. Law and Human Behavior, 40(2), 182-194. doi:10.1037/lhb0000170

Jones, N. J., Brown, S. L., Wanamaker, K. A., \& Greiner, L. E. (2014). A quantitative exploration of gendered pathways to crime in a sample of male and female juvenile offenders. Feminist Criminology, 9(2), 113-136.

doi:10.1177/1557085113501850 
Jung, S., \& Buro, K. (2017). Appraising risk for intimate partner violence in a police context. Criminal Justice and Behavior, 44(2), 240-260. doi: $10.1177 / 0093854816667974$

Justice Health \& Forensic Mental Health, \& Juvenile Justice NSW. (2017). 2015 young people in custody health survey: Full report. Malabar, NSW: Author.

Kafka, M. P. (1997). A monoamine hypothesis for the pathophysiology of paraphilic disorders. Archives of Sexual Behavior, 26(4), 343-358. doi:10.1023/A:1024535201089

Keating, F. (2007). African Caribbean men and mental health. London, UK: Race Equality Foundation.

Keating, F. (2016). Racialized communities, producing madness and dangerousness. Intersectionalities: A Global Journal of Social Work Analysis, Research, Polity, and Practice, 5(3).

Keating, F., Robertson, D., Francis, E., \& McCulloch, A. (2002). Breaking circles of fear: A review of the relationship between African and Caribbean communities and mental health services. London, UK: Sainsbury Centre for Mental Health.

Kemshall, H. (2008). Risks, rights and justice: Understanding and responding to youth risk. Youth Justice, 8(1), 21-37. doi:10.1177/1473225407087040

Kimmel, M. S., \& Mahler, M. (2003). Adolescent masculinity, homophobia, and violence: Random school shootings, 1982-2001. American Behavioral Scientist, 46(10), 1439-1458. doi:10.1177/0002764203046010010

Kline, R. B. (2016). Principles and practice of structural equation modeling (4th ed.) New York, NY: Guilford Press. 
Klonsky, E. D., \& Oltmanns, T. F. (2002). Informant-Reports of personality disorder: Relation to Self-Reports and future research directions. Clinical Psychology: Science and Practice, 9(3), 300-311. doi:10.1093/clipsy.9.3.300

Kovarikova, J. (2017). Exploring youth outcomes after aging-out of care. Toronto, ON: Office of the Provincial Advocate for Children and Youth.

Kraemer, H. C., Measelle, J. R., Ablow, J. C., Essex, M. J., Boyce, W. T., \& Kupfer, D. J. (2003). A new approach to integrating data from multiple informants in psychiatric assessment and research: Mixing and matching contexts and perspectives. American Journal of Psychiatry, 160(9), 1566-1577. doi:10.1176/appi.ajp.160.9.1566

Kramer, T. L., Phillips, S. D., Hargis, M. B., Miller, T. L., Burns, B. J., \& Robbins, J. M. (2004). Disagreement between parent and adolescent reports of functional impairment. Journal of Child Psychology and Psychiatry, 45(2), 248-259. doi:10.1111/j.1469-7610.2004.00217.x

Kuhn, C., Aebi, M., Jakobsen, H., Banaschewski, T., Poustka, L., Grimmer, Y., . . . Steinhausen, H. (2017). Effective mental health screening in adolescents: Should we collect data from youth, parents or both? Child Psychiatry and Human Development, 48(3), 385-392. doi:10.1007/s10578-016-0665-0

Kuster, F., \& Orth, U. (2013). The long-term stability of self-esteem: Its time-dependent decay and nonzero asymptote. Personality and Social Psychology Bulletin, 39(5), 677-690. doi:10.1177/0146167213480189 
Kutcher, S., \& McDougall, A. (2009). Problems with access to adolescent mental health care can lead to dealings with the criminal justice system. Paediatrics \& Child Health, 14(1), 15-18. doi:10.1093/pch/14.1.15

Lader, D., Singleton, N., \& Meltzer H. (2003). Psychiatric morbidity among young offenders in England and Wales. International Review of Psychiatry, 15(1), 144147. doi:10.1080/0954026021000046074

Lahey, B.B., Applegate, B., McBurnett, K., Biederman, J., Greenhill, L., Hynd, G.W., . . . \& Shaffer, D. (1994). DSM-IV field trials for Attention Deficit Hyperactivity Disorder in children and adolescents. American Journal of Psychiatry, 151(11), 1673-85. doi:10.1176/ajp.151.11.1673

Laird, R. D., \& De Los Reyes, A. (2013). Testing informant discrepancies as predictors of early adolescent psychopathology: Why difference scores cannot tell you what you want to know and how polynomial regression may. Journal of Abnormal Child Psychology, 41(1), 1-14. doi:10.1007/s10802-012-9659-y

Laird, R. D., \& Weems, C. F. (2011). The equivalence of regression models using difference scores and models using separate scores for each informant: Implications for the study of informant discrepancies. Psychological Assessment, 23(2), 388-397. doi:10.1037/a0021926

Laishes, J. (2002). The 2002 mental health strategy for female offender. Ottawa: Correctional Service of Canada, Mental Health, Health Services.

Lawes, L. E. (1938). Invisible Stripes. New York, NY: Farrar and Rinehart, Inc. LeBel, T. P. (2012). Invisible stripes? Formerly incarcerated persons' perceptions of stigma. Deviant Behavior, 33(2), 89-107. doi:10.1080/01639625.2010.538365 
LeBel, T. P., Burnett, R., Maruna, S., \& Bushway, S. (2008). The 'Chicken and egg' of subjective and social factors in desistance from crime. European Journal of Criminology, 5(2), 131-159. doi:10.1177/1477370807087640

Lenhard, W., \& Lenhard, A. (2014). Hypothesis tests for comparing correlations. Psychometrica. doi:10.13140/RG.2.1.2954.1367

Lennox, C., Bell, V., O'Malley, K., Shaw, J., \& Dolan, M. (2013). A prospective cohort study of the changing mental health needs of adolescents in custody. $B M J$ Open, 3(3), e002358. doi:10.1136/bmjopen-2012-002358

Lennox, C., O'Malley, K., Bell, V., Shaw, J., \& Dolan, M. (2015). The validity and clinical utility of the Massachusetts youth screening instrument - version 2 (MAYSI-2) in the UK: Validity and utility of the MAYSI-2 in the UK. Criminal Behaviour and Mental Health, 25(3), 207-219. doi:10.1002/cbm.1924

Leschied, A. (2011). Youth justice and mental health in perspective. Healthcare Quarterly, 14(2), 58-63. doi: 10.12927/hcq.2011.22364

Letourneau, E. J., \& Caldwell, M. F. (2013). Expensive, harmful policies that don't work or how juvenile sexual offending is addressed in the U.S. International Journal of Behavioral Consultation and Therapy, 8(3-4), 23-29. doi:10.1037/h0100979

Letourneau, E. J., Harris, A. J., Shields, R. T., Walfield, S. M., Ruzicka, A. E., Buckman, C., , . Nair, R. (2018). Effects of juvenile sex offender registration on adolescent well-being: An empirical examination. Psychology, Public Policy, and Law, 24(1), 105-117. doi:10.1037/law0000155 
Levy, B., Celen-Demirtas, S., Surguladze, T., \& Sweeney, K. K. (2014). Stigma and discrimination: A socio-cultural etiology of mental illness. The Humanistic Psychologist, 42(2), 199-214. doi:10.1080/08873267.2014.893513

Liebenberg, L., \& Ungar, M. (2014). A comparison of service use among youth involved with juvenile justice and mental health. Children and Youth Services Review, 39, 117-122. doi:10.1016/j.childyouth.2014.02.007

Lindert, J., von Ehrenstein, O. S., Grashow, R., Gal, G., Braehler, E., \& Weisskopf, M. G. (2014). Sexual and physical abuse in childhood is associated with depression and anxiety over the life course: Systematic review and metaanalysis. International Journal of Public Health, 59(2), 359-372. doi:10.1007/s00038-013-0519-5

Link, B. G., \& Phelan, J. C. (2001). Conceptualizing stigma. Annual Review of Sociology, 27(1), 363-385. doi:10.1146/annurev.soc.27.1.363

Little, T. D., Lindenberger, U., \& Nesselroade, J. R. (1999). On selecting indicators for multivariate measurement and modeling with latent variables: When "good" indicators are bad and "bad" indicators are good. Psychological Methods, 4(2), 192-211. doi:10.1037/1082-989X.4.2.192

Livingston, J. D., \& Boyd, J. E. (2010). Correlates and consequences of internalized stigma for people living with mental illness: A systematic review and metaanalysis. Social Science \& Medicine, 71(12), 2150-2161. doi:10.1016/j.socscimed.2010.09.030

Livingston, J. D., Rossiter, K. R., \& Verdun-Jones, S. N. (2011). 'Forensic' labelling: An empirical assessment of its effects on self-stigma for people with severe mental 
illness. Psychiatry Research, 188(1), 115-122.

doi:10.1016/j.psychres.2011.01.018

Loeber, R., \& Farrington, D. P. (2012). From juvenile delinquency to adult crime:

Criminal careers, justice policy, and prevention. New York: Oxford University Press.

Loeber, R., Green, S. M., \& Lahey, B. B. (1990). Mental health professional's perception of the utility of children, mothers, and teachers as informants on childhood psychopathology. Journal of Clinical Child Psychology, 19(2). doi:10.1207/s15374424jccp1902_5

Loeber, R., Green, S., Lahey, B. B., \& Stouthamer-Loeber, M. (1989). Optimal informants on childhood disruptive disorders. Development and Psychopathology, 10(4), 317-337. doi:10.1017/S095457940000050X

Longo, R. E., \& Prescott, D. S. (Eds.). (2011). Current perspectives: Working with sexually aggressive youth \& youth with sexual behavior problems. Holyoke, MA: NEARI Press.

Lowry, R. (2019). VasserStats [Computer software]. Retrieved from: http://vassarstats.net

Lu, W., Lindsey, M. A., Irsheid, S., \& Nebbitt, V. E. (2017). Psychometric properties of the CES-D among black adolescents in public housing. Journal of the Society for Social Work and Research, 8(4), 595-619. doi:10.1086/694791

Lysaker, P. H., Roe, D., \& Yanos, P. T. (2007). Toward understanding the insight paradox: Internalized stigma moderates the association between insight and social 
functioning, hope, and self-esteem among people with schizophrenia spectrum disorders. Schizophrenia Bulletin, 33(1), 192-199. doi:10.1093/schbul/sb1016

Maas, C., Herrenkohl, T. I., \& Sousa, C. (2008). Review of research on child maltreatment and violence in youth. Los Angeles, CA: Sage Publications. doi: $10.1177 / 1524838007311105$

Madfis, E. (2014). Triple entitlement and homicidal anger: An exploration of the intersectional identities of american mass murderers. Men and Masculinities, 17(1), 67-86. doi:10.1177/1097184X14523432

Major, B., \& O'Brien, L. T. (2005). The social psychology of stigma. Annual Review of Psychology, 56(1), 393-421. doi:10.1146/annurev.psych.56.091103.070137

Majors, R., \& J. M. Billson. (1992). Cool pose. New York, NY: Lexington Books. Malvaso, C. G., Delfabbro, P. H., Day, A., \& Nobes, G. (2019). Young people under youth justice supervision with varying child protection histories: An analysis of group differences. International Journal of Offender Therapy and Comparative Criminology, 63(2), 159-178. doi:10.1177/0306624X18791735

Maniglio, R. (2015). Significance, nature, and direction of the association between child sexual abuse and conduct disorder: A systematic review. Los Angeles, CA:

SAGE Publications. doi:10.1177/1524838014526068

Mann, M., Hosman, C. M. H., Schaalma, H. P., \& de Vries, N. K. (2004). Self-esteem in a broad-spectrum approach for mental health promotion. Health Education Research, 19(4), 357-372. doi:10.1093/her/cyg041 
Martel, M. M., Markon, K., \& Smith, G. T. (2017). Research review: Multi-informant integration in child and adolescent psychopathology diagnosis. Journal of Child Psychology and Psychiatry, 58(2), 116-128. doi:10.1111/jcpp.12611

Maurutto, P., \& Hannah-Moffat, K. (2006). assembling risk and the restructuring of penal control. The British Journal of Criminology, 46(3), 438-454. doi:10.1093/bjc/azi073

Maurutto, P., \& Hannah-Moffat, K. (2007). Understanding risk in the context of the youth criminal justice act. Canadian Journal of Criminology and Criminal Justice, 49(4), 465-491. doi:10.3138/cjccj.49.4.465

McCall, L. (2005). The complexity of intersectionality. Signs, 30(3), 1771-1800. doi: $10.1086 / 426800$

McClure, Auden C., Tanski, S. E., Kingsbury, J., Gerrard, M., \& Sargent, J. D. (2010). Characteristics associated with low self-esteem among US adolescents. Academic Pediatrics, 10(4), 238-244.e2. doi:10.1016/j.acap.2010.03.007

McConaughy, S.H. (1992). Objective assessment of children's behavioral and emotional problems. In C.E. Walker \& M.C. Roberts (Eds.), Handbook of clinical child psychology (pp. 163-180). New York: Wiley.

McCormick, S., Peterson-Badali, M., \& Skilling, T. A. (2017). The role of mental health and specific responsivity in juvenile justice rehabilitation. Law and Human Behavior, 41(1), 55-67. doi:10.1037/lhb0000228

McCuish, E. C., Cale, J., \& Corrado, R. R. (2017). Abuse experiences of family members, child maltreatment, and the development of sex offending among incarcerated adolescent males: Differences between adolescent sex offenders and 
adolescent non-sex offenders. International Journal of Offender Therapy and Comparative Criminology, 61(2), 127-149. doi:10.1177/0306624X15597492 McCuish, E., Lussier, P., \& Corrado, R. (2016). Criminal careers of juvenile sex and nonsex offenders: Evidence from a prospective longitudinal study. Youth Violence and Juvenile Justice, 14(3), 199-224. doi:10.1177/1541204014567541

McFarlane, K. (2010). From care to custody: Young women in out-of-home care in the criminal justice system. Current Issues in Criminal Justice, 22(2), 345-353. doi:10.1080/10345329.2010.12035890

McNeill, F. (2012). Four forms of 'offender' rehabilitation: Towards an interdisciplinary perspective: Forms of offender rehabilitation. Legal and Criminological Psychology, 17(1), 18-36. doi:10.1111/j.2044-8333.2011.02039.x

Meloy, M., Boatwright, J., \& Curtis, K. (2013). Views from the top and bottom: Lawmakers and practitioners discuss sex offender laws. American Journal of Criminal Justice, 38(4), 616-638. doi:10.1007/s12103-012-9189-8

Mercado, C. C., Alvarez, S., \& Levenson, J. (2008). The impact of specialized sex offender legislation on community reentry. Sexual Abuse: A Journal of Research and Treatment, 20(2), 188-205. doi:10.1177/1079063208317540

Merikangas, K. R., Ph.D., He, J., M.Sc., Burstein, M., Ph.D., Swanson, S. A., Sc.M., Avenevoli, S., Ph.D., Cui, L., M.Sc., . . Swendsen, J., Ph.D. (2010). Lifetime prevalence of mental disorders in U.S. adolescents: Results from the national comorbidity survey Replication-Adolescent supplement (NCS-A). Journal of the American Academy of Child \& Adolescent Psychiatry, 49(10), 980-989. doi:10.1016/j.jaac.2010.05.017 
Messerschmidt, J. W. (1993). Masculinities and crime: Critique and reconceptualization of theory. Lanham, MD: Rowman \& Littlefield.

Messerschmidt, J. W. (1999). Making bodies matter: Adolescent masculinities, the body, and varieties of violence. Theoretical Criminology, 3(2), 197-220. doi: $10.1177 / 1362480699003002004$

Messerschmidt, J. W. (2004). Flesh and blood: Adolescent gender diversity and violence. Lanham, MD: Rowman \& Littlefield Publishers.

Miladinovic, Z. (2016). Youth court statistics in Canada, 2014/2015. Juristat. Statistics Canada Catalogue no. 85-002-X. Ottawa, ON: Statistics Canada.

Miller, A. B., Esposito-Smythers, C., \& Leichtweis, R. N. (2015). Role of social support in adolescent suicidal ideation and suicide attempts. Journal of Adolescent Health, 56(3), 286-292. doi:10.1016/j.jadohealth.2014.10.265

Miner, M. H., Robinson, B. E., Knight, R. A., Berg, D., Romine, R., \& Netland, J. (2010). Understanding sexual perpetration against children: Effects of attachment style, interpersonal involvement, and hypersexuality. Sexual Abuse: A Journal of Research and Treatment, 22(1), 58-77. doi:10.1177/1079063209353183

Mingus, W., \& Burchfield, K. B. (2012). From prison to integration: Applying modified labeling theory to sex offenders. Criminal Justice Studies, 25(1), 97-109. doi:10.1080/1478601X.2012.657906

Ministry of Children and Youth Services. (2015). Youth justice outcomes framework. Toronto, ON: Author.

Mitchell, O., \& Caudy, M. S. (2015). Examining racial disparities in drug arrests. Justice Quarterly, 32(2), 288-313. doi:10.1080/07418825.2012.761721 
Monahan, J., \& Skeem, J. L. (2016). Risk assessment in criminal sentencing. Annual Review of Clinical Psychology, 12(1), 489-513.

Monterastelli, S. (2017). Every hand's a loser: The intersection of zero-tolerance policies, mental illness in children and adolescents, and the juvenile justice system. Law and Psychology Review, 41, 209.

Monto, M., Zgourides, G., \& Harris, R. (1998). Empathy, self-esteem, and the adolescent sexual offender. Sexual Abuse: A Journal of Research and Treatment, 10(2), $127-$ 140. doi:10.1023/A:1022019532573

Moore, K. E., \& Tangney, J. P. (2017). Managing the concealable stigma of criminal justice system involvement: A longitudinal examination of anticipated stigma, social withdrawal, and post-release adjustment: Managing the concealable stigma. Journal of Social Issues, 73(2), 322-340. doi:10.1111/josi.12219

Moore, K. E., Milam, K. C., Folk, J. B., \& Tangney, J. P. (2018). Self-stigma among criminal offenders: Risk and protective factors. Stigma and Health, 3(3), 241-252. doi:10.1037/sah0000092

Moore, K. E., Tangney, J. P., \& Stuewig, J. B. (2016). The self-stigma process in criminal offenders. Stigma and Health, 1(3), 206-224. doi:10.1037/sah0000024

Morais, H. B., Alexander, A. A., Fix, R. L., \& Burkhart, B. R. (2018). Childhood sexual abuse in adolescents adjudicated for sexual offenses: Mental health consequences and sexual offending behaviors. Sexual Abuse: A Journal of Research and Treatment, 30(1), 23-42. doi:10.1177/1079063215625224

Morgan, K. D. (1993). Factors influencing probation outcomes: A review of the literature. Federal Probation, 57(2), 23-29. 
Mosher, C. J. (1998). Discrimination and denial: Systemic racism in Ontario's legal and criminal justice systems, 1892-1961. Toronto, ON: University of Toronto Press.

Mossman, D. (2015). From group data to useful probabilities: The relevance of actuarial risk assessment in individual instances. The Journal of the American Academy of Psychiatry and the Law, 43(1), 93.

Moylan, C. A., Herrenkohl, T. I., Sousa, C., Tajima, E. A., Herrenkohl, R. C., \& Russo, M. J. (2010). The effects of child abuse and exposure to domestic violence on adolescent internalizing and externalizing behavior problems. Journal of Family Violence, 25(1), 53-63. doi:10.1007/s10896-009-9269-9

Myburgh, J-E., Camman, C., \& Wormith, J. S. (2015). Review of pretrial risk assessment and factors predicting pretrial release failure. Saskatoon, SK: University of Saskatchewan Centre for Forensic Behavioural Science and Justice Studies.

Najman, J. M., Williams, G. M., Nikles, J., Spence, S., Bor, W., O'Callaghan, M., . . Shuttlewood, G. J. (2001). Bias influencing maternal reports of child behaviour and emotional state. Social Psychiatry and Psychiatric Epidemiology, 36(4), 186194. doi:10.1007/s001270170062

National Research Council. (2013). Reforming juvenile justice: A developmental approach. Washington, DC: The National Academies Press. https://doi.org.10.17226/14685

Nelemans, S. A., Branje, S. J. T., Hale, W., Goossens, L., Koot, H. M., Oldehinkel, A. J., \& Meeus, W. H. J. (2016). Discrepancies between perceptions of the ParentAdolescent relationship and early adolescent depressive symptoms: An 
illustration of polynomial regression analysis. Journal of Youth and Adolescence, 45(10), 2049-2063.

Nelson, R. J., \& Vincent, G. M. (2018). Matching services to criminogenic needs following comprehensive risk assessment implementation in juvenile probation. Criminal Justice and Behavior, 45(8), 1136-1153. doi: $10.1177 / 0093854818780923$

NeMoyer, A., Goldstein, N. E. S., McKitten, R. L., Prelic, A., Ebbecke, J., Foster, E., \& Burkard, C. (2014). Predictors of juveniles' noncompliance with probation requirements. Law and Human Behavior, 38(6), 580-591. doi:10.1037/lhb0000083

Newton-Howes, G., \& Mullen, R. (2011). Coercion in psychiatric care: systematic review of correlates and themes psychiatric services. Psychiatry Services, 65(5), 465-470. doi: 10.1176/ps.62.5.pss6205_0465.

Nhan, J., Polzer, K., \& Ferguson, J. (2012). “More dangerous than hitmen”: Judicial perceptions of sexual offenders. International Journal of Criminology and Sociological Theory, 5(1), 823-836.

Norman, R. E., Byambaa, M., De, R., Butchart, A., Scott, J., \& Vos, T. (2012). The longterm health consequences of child physical abuse, emotional abuse, and neglect: A systematic review and meta-analysis. PLoS Medicine, 9(11), e1001349. doi:10.1371/journal.pmed.1001349

Nowacki, J. S. (2017). An intersectional approach to race/ethnicity, sex, and age disparity in federal sentencing outcomes: An examination of policy across time 
periods. Criminology \& Criminal Justice, 17(1), 97-116.

doi:10.1177/1748895816642502

O'Donnell, P. C., \& Lurigio, A. J. (2008). Psychosocial predictors of clinicians' recommendations and judges' placement orders in a juvenile court. Criminal Justice and Behavior, 35(11), 1429-1448. doi:10.1177/0093854808324061

Office of Juvenile Justice and Delinquency Prevention. (2000). Juvenile Justice Journal, II (1). Washington, DC: US Department of Justice, Office of Juvenile Justice and Delinquency Prevention.

Office of the Child and Youth Advocate. (2015). More care less court: Keeping youth out of the criminal justice system. Fredericton, NB: Author.

Office of the Correctional Investigator, \& Ontario Office of the Provincial Advocate for Children and Youth. (2017). Missed Opportunities: The Experience of Young Adults Incarcerated in Federal Penitentiaries. Ottawa, ON: Office of the Correctional Investigator.

Office of the Correctional Investigator. (2014). Annual report of the Office of the Correctional Investigator, 2013-2014. Ottawa, ON: Author.

Olver, M. E., Stockdale, K. C., \& Wormith, J. S. (2009). Risk assessment with young offenders: A meta-analysis of three assessment measures. Criminal Justice and Behavior, 36(4), 329-353. doi:10.1177/0093854809331457

Ontario Federation of Indigenous Friendship Centres. (2016). USAI Framework: Utility self-voicing access inter-relationality. Toronto, ON: Author.

Orth, U., \& Robins, R. W. (2014). The development of self-esteem. Current Directions in Psychological Science, 23(5), 381-387. doi:10.1177/0963721414547414 
Ottati, V., Bodenhausen, G. V., \& Newman, L. S. (2005). Social psychological models of mental illness stigma In P. W. Corrigan (Ed.), On the stigma of mental illness: practical strategies for research and social change (pp. 99-128). Washington, DC: American Psychological Association. doi:10.1037/10887-004

Pachankis, J. E. (2007). The psychological implications of concealing a stigma: A cognitive-affective-behavioral model. Psychological Bulletin, 133(2), 328-345. doi:10.1037/0033-2909.133.2.328

Papalia, N., Ogloff, J. R. P., Cutajar, M., \& Mullen, P. E. (2018). Child sexual abuse and criminal offending: Gender-specific effects and the role of abuse characteristics and other adverse outcomes. Child Maltreatment, 23(4), 399-416. doi:10.1177/1077559518785779

Paré, M. (2012). Examining Children's Participation as a Right Protected under Canadian Law. In E. Murray (Ed.), Children Matter - Exploring Child and Youth Human Rights Issues in Canada [E-book]. Calgary, AB: Mount Royal University.

Paré, M. (2017). Children’s rights are human rights and why Canadian implementation lags behind. Canadian Journal of Children's Rights, 4(1), 24-47.

Parker, R., \& Aggleton, P. (2003). HIV and AIDS-related stigma and discrimination: A conceptual framework and implications for action. Social Science \& Medicine, 57(1), 13-24. doi:10.1016/S0277-9536(02)00304-0

Pearson, L. (2015). A Canada fit for children 2015: Identity, rights, and belonging. Ottawa, ON: Landon Pearson Resource Centre for the Study of Childhood and Children's Rights. 
Peltonen, K., Ellonen, N., Larsen, H. B., \& Helweg-Larsen, K. (2010). Parental violence and adolescent mental health. European Child \& Adolescent Psychiatry, 19(11), 813-822. doi:10.1007/s00787-010-0130-8

Penner, E. K., Roesch, R., \& Viljoen, J. L. (2011). Young offenders in custody: An international comparison of mental health services. International Journal of Forensic Mental Health, 10(3), 215-232. doi:10.1080/14999013.2011.598427

Penney, S. R., \& Skilling, T. A. (2012). Moderators of informant agreement in the assessment of adolescent psychopathology: Extension to a forensic sample. Psychological Assessment, 24(2), 386-401. doi:10.1037/a0025693

Peterson-Badali, M., McCormick, S., Vitopoulos, N., Davis, K., Haqanee, Z., \& Skilling, T. A. (2015). Mental health in the context of Canada's youth justice system. Canadian Criminal Law Review, 19(1), 5.

Peterson-Badali, M., Skilling, T., \& Haqanee, Z. (2015). Examining implementation of risk assessment in case management for youth in the justice system. Criminal Justice and Behavior, 42(3), 304-320. doi:10.1177/0093854814549595

Petitclerc, A., Briggs-Gowan, M. J., Estabrook, R., Burns, J. L., Anderson, E. L., McCarthy, K. J., \& Wakschlag, L. S. (2015). Contextual variation in young children's observed disruptive behavior on the DB-DOS: Implications for early identification. Journal of Child Psychology and Psychiatry, 56(9).

Pincus, F. L. (1996). Discrimination comes in many forms: Individual, institutional, and structural. American Behavioral Scientist, 40(2), 186-194. doi:10.1177/0002764296040002009 
Pincus, F.L. (1999). From individual to structural discrimination In H. J. Ehrlich (Ed.), Race and Ethnic Conflict: Contending Views on Prejudice, Discrimination, and Ethnoviolence (pp.120-124). Boulder, CO: Westview Press.

Polaschek, D. L. L. (2012). An appraisal of the risk-need-responsivity (RNR) model of offender rehabilitation and its application in correctional treatment: The RNR model of offender rehabilitation: An appraisal. Legal and Criminological Psychology, 17(1), 1-17. doi:10.1111/j.2044-8333.2011.02038.x

Pollack, S. (2007). "I'm just not good in relationships": Victimization discourses and the gendered regulation of criminalized women. Feminist Criminology, 2(2), 158174. doi: $10.1177 / 1557085106297521$

Potter, H. (2013). Intersectional criminology: Interrogating identity and power in criminological research and theory. Critical Criminology, 21(3), 305-318. doi:10.1007/s10612-013-9203-6

Public Safety Canada. (2017). Research highlights: Youth mental health, mental illness \& crime. Ottawa, ON: Author.

Pusch, N., \& Holtfreter, K. (2018). Gender and risk assessment in juvenile offenders: A meta-analysis. Criminal Justice and Behavior, 45(1), 56-81. doi: $10.1177 / 0093854817721720$

Quinsey, V. L., Skilling, T. A., Lalumière, M. L., \& Craig, W. M. (2004). Juvenile delinquency: Understanding the origins of individual differences. Washington, DC: American Psychological Association. doi:10.1037/10623-000

Rawal, P., Romansky, J., Jenuwine, M., \& Lyons, J. S. (2004). Racial differences in the mental health needs and service utilization of youth in the juvenile justice 
system. The Journal of Behavioral Health Services \& Research, 31(3), 242-254. doi:10.1007/BF02287288

Renk, K. (2005). Cross-informant ratings of the behavior of children and adolescents: The "Gold standard". Journal of Child and Family Studies, 14(4), 457-468. doi:10.1007/s10826-005-7182-2

Renk, K., \& Phares, V. (2004). Cross-informant ratings of social competence in children and adolescents. Clinical Psychology Review, 24(2), 239-254. doi:10.1016/j.cpr.2004.01.004

Renzetti, C. (2013). Feminist Criminology. New York, NY: Routledge.

Reynolds, E. K., MacPherson, L., Matusiewicz, A. K., Schreiber, W. M., \& Lejuez, C. W. (2011). Discrepancy between mother and child reports of parental knowledge and the relation to risk behavior engagement. Journal of Clinical Child \& Adolescent Psychology, 40(1), 67-79. doi:10.1080/15374416.2011.533406

Ricciardelli, R., \& Moir, M. (2013). Stigmatized among the stigmatized: Sex offenders in Canadian penitentiaries. Canadian Journal of Criminology and Criminal Justice, 55(3), 353-385. doi:10.3138/cjccj.2012.E22

Richardson, J. E. (2007). Analysing newspapers: An approach from critical discourse analysis. New York, NY: Palgrave Macmillan.

Ritsher, J. B., Otilingam, P. G., \& Grajales, M. (2003). Internalized stigma of mental illness: psychometric properties of a new measure. Psychiatry Research, 121, 3149. doi:10.1016/j.psychres.2003.08.008 
Robbers, M. L. P. (2009). Lifers on the outside: Sex offenders and disintegrative shaming. International Journal of Offender Therapy and Comparative Criminology, 53(1), 5-28. doi:10.1177/0306624X07312953

Rocque, M., \& Snellings, Q. (2018). The new disciplinology: Research, theory, and remaining puzzles on the school-to-prison pipeline. Journal of Criminal Justice, 59, 3-11. doi:10.1016/j.jcrimjus.2017.05.002

Rogers, L. O., Scott, M. A., \& Way, N. (2015). Racial and gender identity among black adolescent males: An intersectionality perspective. Child Development, 86(2), 407-424. doi:10.1111/cdev.12303

Romano, E., \& De Luca, R. V. (2001). Male sexual abuse: A review of effects, abuse characteristics, and links with later psychological functioning. Aggression and Violent Behavior, 6(1), 55-78. doi:10.1016/S1359-1789(99)00011-7

Rosenberg, M. (1989). Society and the Adolescent Self-Image (Revised). Middletown, CT: Wesleyan University Press.

Royer-Gagnier, Skilling, T.A., Brown, S.L., Moore, T., \& Rawana, J. (2016). The Strength Assessment Inventory - Youth Version: An evaluation of the psychometric properties with male and female justice-involved youth. Psychological Assessment, 28, 563-574. doi: 10.1037/pas0000199

Rubio-Stipec, M., Fitzmaurice, G., Murphy, J., \& Walker, A. (2003). The use of multiple informants in identifying the risk factors of depressive and disruptive disorders: Are they interchangeable? Social Psychiatry and Psychiatric Epidemiology, 38(2), 51-58. doi:10.1007/s00127-003-0600-0 
Ryan, G., Lane, S. L., \& Leversee, T. F. (2010). Juvenile sexual offending: Causes, consequences, and correction (3rd ed.) Hoboken, N. J.: John Wiley \& Sons.

Saegert, S. C., Adler, N. E., Bullock, H. E., Cauce, A. M., Lui, W. M., \& Wyche, K. F. (2007). Report of the APA task force report on socioeconomic status. Washington, DC: American Psychological Association.

Safren, S. A., \& Pantalone, D. W. (2006). Social anxiety and barriers to resilience among lesbian, gay, and bisexual adolescents. In A. M. Omoto \& H. S. Kurtzman (Eds.), Sexual orientation and mental health: Examining identity and development in lesbian, gay, and bisexual people (pp.55-71). Washington, DC: American Psychological Association

Salekin, R. T. (2015). Forensic evaluation and treatment of juveniles: Innovation and best practice. Washington, D.C: American Psychological Association.

Salole, A. T., \& Abdulle, Z. (2015). Quick to punish: An examination of the school to prison pipeline for marginalized youth. Canadian Review of Social Policy, (72/73), 124.

Sample, L. L., \& Kadleck, C. (2008). Sex offender laws: Legislators' accounts of the need for policy. Criminal Justice Policy Review, 19(1), 40-62. doi: $10.1177 / 0887403407308292$

Sapp, A. D., \& Vaughn, M. S. (1990). The social status of adult and juvenile sex offenders in prison: An analysis of the importation model. Journal of Police and Criminal Psychology, 6(2), 2-7. doi:10.1007/BF02806591

SCC (2012). A.C. v. Manitoba (Director of Child and Family Services, [2009] 2 S.C.R.181 
Schubert, C. A., Mulvey, E. P., \& Glasheen, C. (2011). Influence of mental health and substance use problems and criminogenic risk on outcomes in serious juvenile offenders. Journal of the American Academy of Child \& Adolescent Psychiatry, 50(9), 925-937. doi:10.1016/j.jaac.2011.06.006

Schwalbe, C. S. (2012). Toward an integrated theory of probation. Criminal Justice and Behavior, 39 (2), 185-201. doi:10.1177/0093854811430185

Schwalbe, C. S., \& Maschi, T. (2008). Investigating probation strategies with juvenile offenders: The influence of officers' attitudes and youth characteristics. Law and Human Behavior, 33(5), 357-367. doi:10.1007/s10979-008-9158-4

Schwalbe, C. S., Hatcher, S. S., \& Maschi, T. (2009). The effects of treatment needs and prior social services use on juvenile court decision making. Social Work Research, 33(1), 31-40. doi:10.1093/swr/33.1.31

Scully, B. M., \& Finlay, J. (2015). Cross-over youth: Care to custody. Ottawa, ON: Youth Justice Canada.

Seidler, K. (2010). Community management of sex offenders: stigma versus support. Sexual Abuse in Australia and New Zealand, 2(2), 66-76.

Seiter, L. (2017). Mental health and juvenile justice: A review of prevalence, promising practices, and areas for improvement. Washington, DC: National Technical Assistance Center for the Education of Neglected or Delinquent Children and Youth.

Semel, R. A. (2017). Utility of the ASEBA Youth Self-Report 9YSR) in juvenile delinquency assessments. EC Psychology and Psychiatry, 1(6), 217-225. 
Seto, M. C., \& Lalumière, M. L. (2010). What is so special about male adolescent sexual offending? A review and test of explanations through metaanalysis. Psychological Bulletin, 136(4), 526-575. doi:10.1037/a0019700

Sibley, M. H., Campez, M., \& Raiker, J. S. (2017). Re-examining ADHD-related selfreporting problems using polynomial regression. Assessment, 1-10. doi: $10.1177 / 1073191117693349$

Silva, T. C., Graña, J. L., \& González-Cieza, L. (2014). Self-reported physical and emotional abuse among youth offenders and their association with internalizing and externalizing psychopathology: A preliminary study. International Journal of Offender Therapy and Comparative Criminology, 58(5), 590-606. doi:10.1177/0306624X12474975

Simon, J. (1998). Managing the monstrous: Sex offenders and the new penology. Psychology, Public Policy, and Law, 4(1-2), 452-467.

Singer, D., \& Hunter, M. (1999). The experience of premature menopause: A thematic discourse analysis. Journal of Reproductive and Infant Psychology, 17(1), 63-81. doi:10.1080/02646839908404585

Skeem, J. L., Manchak, S., \& Peterson, J. K. (2011). Correctional policy for offenders with mental illness: Creating a new paradigm for recidivism reduction. Law and Human Behavior, 35(2), 110-126. doi:10.1007/s10979-010-9223-7

Skilling, T. A., Doiron, J. M., \& Seto, M. C. (2011). Exploring differences in youth and parent reports of antisociality among adolescent sexual and nonsexual offenders. Psychological Assessment, 23(1), 153-163. doi:10.1037/a0021229 
Smiley, C., \& Fakunle, D. (2016). From "brute" to "thug:" the demonization and criminalization of unarmed black male victims in America. Journal of Human Behavior in the Social Environment, 26(3-4), 350. doi:10.1080/10911359.2015.1129256

Smith, S. R. (2007). Making sense of multiple informants in child and adolescent psychopathology: A guide for clinicians. Journal of Psychoeducational Assessment, 25(2), 139-149. doi:10.1177/0734282906296233

Spencer, D. (2009). Sex offender as Homo Sacer. Punishment \& Society, 11(2), 219-240. doi: $10.1177 / 1462474508101493$

Spinney, E., Yeide, M., Feyerherm, W., Cohen, M., Stephenson, R., \& Thomas, C. (2016). Racial disparities in referrals to mental health and substance abuse services from the juvenile justice system: A review of the literature. Journal of Crime and Justice, 39(1), 153-173. doi:10.1080/0735648X.2015.1133492

Sprott, J. B., \& Myers, N. M. (2011). Set up to fail: The unintended consequences of multiple bail conditions. Canadian Journal of Criminology and Criminal Justice, 53(4), 404-423. doi:10.3138/cjccj.53.4.404

Statistics Canada. (2017). Low-income measure, after tax (LIM-AT). Ottawa, ON: Auhtor.

Statistics Canada. (2019). Reference materials, 2016 Census. Ottawa, ON: Author. Steinberg, L. (2009). Adolescent development and juvenile justice. Annual Review of Clinical Psychology, 5(1), 459-485. doi:10.1146/annurev.clinpsy.032408.153603 
Stevelink, S. A. M., Wu, I. C., Voorend, G. N., \& van Brakel, W. H. (2012). The psychometric assessment of internalized stigma instruments: A systematic review. Stigma Research and Action, 2(2), 100-118. doi:10.5463/SRA.v1i1.11

Stuart H (2008). Building an evidence base for anti-stigma programming In J. ArboledaFlórez, \& N. Sartorius (Eds.), Understanding the Stigma of Mental Illness: Theory and Interventions (pp. 135-146). Chichester, UK: John Wiley \& Sons.

Tackett, J. L., Herzhoff, K., Reardon, K. W., Smack, A. J., \& Kushner, S. C. (2013). The relevance of informant discrepancies for the assessment of adolescent personality pathology. Clinical Psychology: Science and Practice, 20(4), 378-392. doi:10.1111/cpsp.12048

Taxman, F. S. (2014). Second generation of RNR: The importance of systemic responsivity in expanding core principles of responsivity. Federal Probation, 78(2), 32.

Taylor, C. (2016). Review of the youth justice system in England and Wales. London, UK: United Kingdom Ministry of Justice.

Taylor, G. W., \& Ussher, J. M. (2001). Making sense of S\&M: A discourse analytic account. Sexualities, 4(3), 293-314. doi:10.1177/136346001004003002

Taylor, J. J., Grant, K. E., Zulauf, C. A., Fowler, P. J., Meyerson, D. A., \& Irsheid, S. (2018). Exposure to community violence and the trajectory of internalizing and externalizing symptoms in a sample of low-income urban youth. Journal of Clinical Child \& Adolescent Psychology, 47(3), 421-435. doi:10.1080/15374416.2016.1152553 
Teplin, L. A., Abram, K. M., McClelland, G. M., Dulcan, M. K., \& Mericle, A. A. (2002). Psychiatric disorders in youth in juvenile detention. Archives of General Psychiatry, 59(12), 1133-1143. doi:10.1001/archpsyc.59.12.1133

Teplin, L. A., Welty, L. J., Abram, K. M., Dulcan, M. K., \& Washburn, J. J. (2012). Prevalence and persistence of psychiatric disorders in youth after detention: A prospective longitudinal study. Archives of General Psychiatry, 69(10), 10311043. doi:10.1001/archgenpsychiatry.2011.2062

Teplin, L. A., Welty, L. J., Abram, K. M., Dulcan, M. K., Washburn, J. J., McCaoy, K., \& Stokes, M. L. (2015). Psychiatric disorders in youth after detention. Washington, D.C.: Office of Juvenile Justice and Delinquency Prevention.

Tewksbury, R. (2005). Collateral consequences of sex offender registration. Journal of Contemporary Criminal Justice, 21(1), 67-81. doi:10.1177/1043986204271704

Tewksbury, R. (2013). Sex offenders and campus-based sex offender registration: Stigma, vulnerability, isolation, and the classroom as refuge. Journal of Qualitative Criminal Justice and Criminology, 1(2), 221-242.

Thanner, M. H., \& Taxman, F. S. (2003). Responsivity: The value of providing intensive services to high-risk offenders. Journal of Substance Abuse Treatment, 24(2), 137-147. doi:10.1016/S0740-5472(02)00352-5

The Royal Australasian College of Physicians. (2011). The health and well-being of incarcerated adolescents. Sydney, AUS: Author.

The Sentencing Project. (2018). Regarding racial disparities in the United States criminal justice system. Washington, DC: Author. 
Thomas, J. (2009). Youth court statistics, 2006/2007. Juristat, 28(4). Statistics Canada Catalogue no. 85-002-X. Ottawa, ON: Statistics Canada.

Thornicroft, G., Rose, D., Kassam, A., \& Sartorius, N. (2007). Stigma: Ignorance, prejudice or discrimination? The British Journal of Psychiatry, 190(3), 192-193. doi:10.1192/bjp.bp.106.025791

Underwood, L. A., \& Washington, A. (2016). Mental illness and juvenile offenders. International Journal of Environmental Research and Public Health, 13 (2), 228. doi:10.3390/ijerph13020228

United Nations General Assembly. (1989). United Nations Convention on the Rights of the Child. New York, NY: United Nations.

Valo, S., \& Tannock, R. (2010). Diagnostic instability of DSM-IV ADHD subtypes: Effects of informant source, instrumentation, and methods for combining symptom reports. Journal of Clinical Child \& Adolescent Psychology, 39(6), 749. $10.1080 / 15374416.2010 .517172$

Van Damme, L., Colins, O. F., \& Vanderplasschen, W. (2014). Gender differences in psychiatric disorders and clusters of self-esteem among detained adolescents. Psychiatry Research, 220(3), 991-997. doi:10.1016/j.psychres.2014.10.012

Varma, K., \& Leroux, E. J. (in press). Understanding the nature and scope of adolescent sexual offending across Canada using Uniform Crime Reporting data. Annual Review of Interdisciplinary Justice Research, 8.

Verhulst, F. C., \& van der Ende, J. (1991). Assessment of child psychopathology: Relationships between different methods, different informants and clinical 
judgment of severity. Acta Psychiatrica Scandinavica, 84(2), 155-159.

doi:10.1111/j.1600-0447.1991.tb03120.x

Verloo, M. M. T. (2006). Multiple inequalities, intersectionality and the european union. The European Journal of Women's Studies, 13(3), 211-228. doi: $10.1177 / 1350506806065753$

Vermeiren, R., Schwab-Stone, M., Ruchkin, V., De Clippele, A., \& Deboutte, D. (2002). Predicting recidivism in delinquent adolescents from psychological and psychiatric assessment. Comprehensive Psychiatry, 43(2), 142-149. doi:10.1053/comp.2002.30809

Vidal, S., \& Woolard, J. (2016). Parents' perceptions of juvenile probation: Relationship and interaction with juvenile probation officers, parent strategies, and youth's compliance on probation. Children and Youth Services Review, 66, 1-8. doi:10.1016/j.childyouth.2016.04.019

Vidal, S., \& Woolard, J. (2017). Youth's perceptions of parental support and parental knowledge as moderators of the association between youth-probation officer relationship and probation non-compliance. Journal of Youth and Adolescence, 46(7), 1452. doi:10.1007/s10964-015-0368-z

Vierhaus, M., Rueth, J. E., \& Lohaus, A. (2016). Parents' perceived similarity to their children, and parents' perspective taking efforts: Associations of cross-informant discrepancies with adolescent problem behavior. Frontiers in Psychology, 7, 367. doi:10.3389/fpsyg.2016.00367 
Viljoen, J. L., Mordell, S., \& Beneteau, J. L. (2012). Prediction of adolescent sexual reoffending: A meta-analysis of the J-SOAP-II, ERASOR, J-SORRAT-II, and static-99. Law and Human Behavior, 36(5), 423-438. doi:10.1037/h0093938

Viljoen, J. L., Mordell, S., \& Beneteau, J. L. (2012). Prediction of adolescent sexual reoffending: A meta-analysis of the J-SOAP-II, ERASOR, J-SORRAT-II, and static-99. Law and Human Behavior, 36(5), 423-438. doi:10.1037/h0093938

Vincent, G. M., Chapman, J., \& Cook, N. E. (2011). Risk-needs assessment in juvenile justice: Predictive validity of the SAVRY, racial differences, and the contribution of needs factors. Criminal Justice and Behavior, 38(1), 42-62.

doi:10.1177/0093854810386000

Vreugdenhil, C., Doreleijers, T. A. H., Vermeiren, R., Wouters, Luuk F. J. M, \& van den Brink, W. (2004). Psychiatric disorders in a representative sample of incarcerated boys in the Netherlands. Journal of the American Academy of Child and Adolescent Psychiatry, 43(1), 97-104. doi:10.1097/00004583-200401000-00019

Vreugdenhil, C., van den Brink, W., Ferdinand, R., Wouters, L., \& Doreleijers, T. (2006). The ability of YSR scales to predict DSM/DISC-C psychiatric disorders among incarcerated male adolescents. European Child \& Adolescent Psychiatry, 15(2), 88-96. doi:10.1007/s00787-006-0497-8

Wald, J., \& Losen, D. J. (2003). Defining and redirecting a school-to-prison pipeline. New Directions for Youth Development, 2003(99), 9-15. doi:10.1002/yd.51

Ward, T., \& Beech, A. (2006). An integrated theory of sexual offending. Aggression and Violent Behavior, 11(1), 44-63. doi:10.1016/j.avb.2005.05.002 
Ward, T., \& Siegert, R. J. (2002). Toward a comprehensive theory of child sexual abuse: A theory knitting perspective. Psychology, Crime \& Law, 8(4), 319-351. doi:10.1080/10683160208401823

Warde, B. (2013). Black male disproportionality in the criminal justice systems of the USA, Canada, and England: A comparative analysis of incarceration. Journal of African American Studies, 17(4), 461-479. doi:10.1007/s12111-012-9235-0

Wibbelink, C. J. M., Hoeve, M., Stams, G. J. J. M., \& Oort, F. J. (2017). A meta-analysis of the association between mental disorders and juvenile recidivism. Aggression and Violent Behavior, 33, 78-90. doi:10.1016/j.avb.2017.01.005

Williams, S. L., \& Fredrick, E. G. (2015). One size may not fit all: The need for a more inclusive and intersectional psychological science on stigma. Sex Roles, 73(9), 384-390. doi:10.1007/s11199-015-0491-z

Winnick, T. A., \& Bodkin, M. (2008). Anticipated stigma and stigma management among those to be labeled "ex-con". Deviant Behavior, 29, 295-333.

Wodak, R., \& Meyer, M. (2009). Methods of critical discourse analysis. London: Sage.

Wright, R. G. (Ed). (2015). Sex offender laws: Failed policies, new directions. New York, NY: Springer Publishing Company, LLC.

Yang, L. H., Kleinman, A., Link, B. G., Phelan, J. C., Lee, S., \& Good, B. (2007). Culture and stigma: Adding moral experience to stigma theory. Social Science \& Medicine, 64(7), 1524-1535. doi:10.1016/j.socscimed.2006.11.013

Young Offenders Act, R.S.C., 1985, c. Y-1.

Young, I. M. (1990/2011). Justice and the politics of difference. Princeton, NJ: Princeton University Press. 
Young, S., Moss, D., Sedgwick, O., Fridman, M., \& Hodgkins, P. (2015). A metaanalysis of the prevalence of attention deficit hyperactivity disorder in incarcerated populations. Psychological Medicine, 45(2), 247-258. doi:10.1017/S0033291714000762

Youngstrom, E., Loeber, R., \& Stouthamer-Loeber, M. (2000). Patterns and correlates of agreement between parent, teacher, and male adolescent ratings of externalizing and internalizing problems. Journal of Consulting and Clinical Psychology, 68(6), 1038-1050. doi:10.1037/0022-006X.68.6.1038

Youth Criminal Justice Act, S.C. 2002, c. 1.

Youth Justice Board for England and Wales. (2016). Annual report and accounts 20152016. London, UK: Author.

Zimet, G. D., Dahlem, N. W., Zimet, S. G., \& Farley, G. K. (1988). The Multidimensional Scale of Perceived Support. Journal of Personality Assessment, 52(1), 30-41. doi:10.1207/s15327752jpa5201_2

Zimet, G. D., Powell, S. S., Farley, G. K., Werkman, S., \& Berkoff, K. A. (1990). Psychometric characteristics of the multidimensional scale of perceived social support. Journal of Personality Assessment, 55(3-4), 610-617. doi:10.1207/s15327752jpa5503\&4_17

Zimmerman, M. A., Copeland, L. A., Shope, J. T., \& Dielman, T. E. (1997). A longitudinal study of self-esteem: Implications for adolescent development. Journal of Youth and Adolescence, 26(2), 117-141. doi:10.1023/A:1024596313925 
Zimring, F. E. (2004). An American travesty: Legal responses to adolescent sexual offending. Chicago, IL: The University of Chicago Press. 


\section{Appendix A}

\section{Sample Copy of Youth Self-Report}

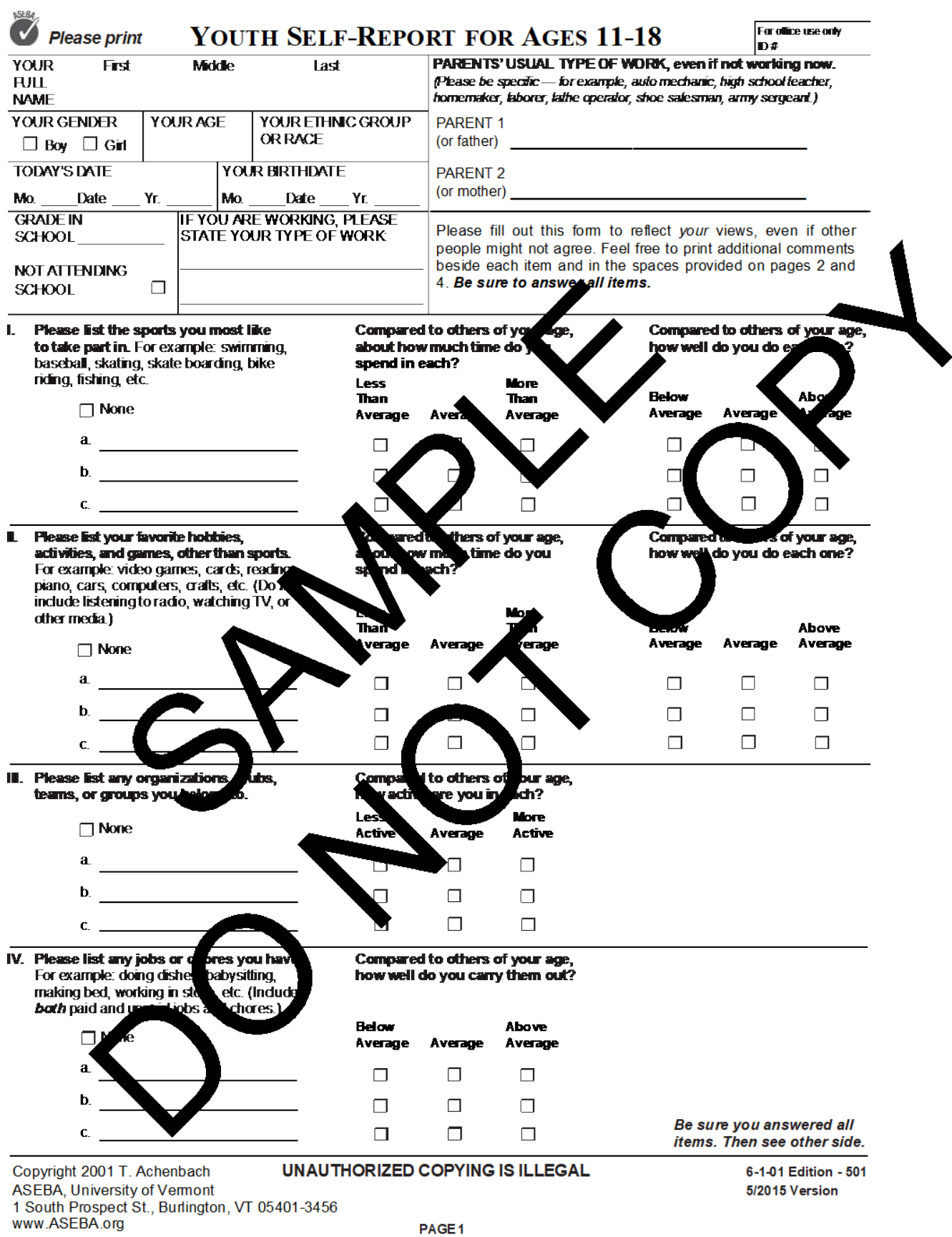


Please print. Be sure to answer all items.

v. 1. About how many close friends do you have? (Do not include brothers \& sisters)

$$
\square \text { None } \square 1 \quad \beth_{2} \text { or } 3 \quad \square_{4} \text { or more }
$$

2. About how many times a week do you do things with any frlends outsidc of regular school hours? (Do not include brothers \& sisters)
$\square$ Less than 1
$\beth_{1}$ or $2 \quad \square 3$ or more

VI. Compared to others of your age, how well do you:

a. Get aiong with your brothers \& sisters?
b. Get along with other kids?
c. Gc: along with your parents?
d. Do things by yourself?

VII. 1. Performance in academic subjects.

$\square$ I do not attend school

$\begin{array}{cccc}\text { Worse } & \text { Average } & \text { Better } & \\ \neg & \sqcap & \neg & \text { I have no brothers or sisters } \\ \sqsupset & \square & \sqsupset & \end{array}$

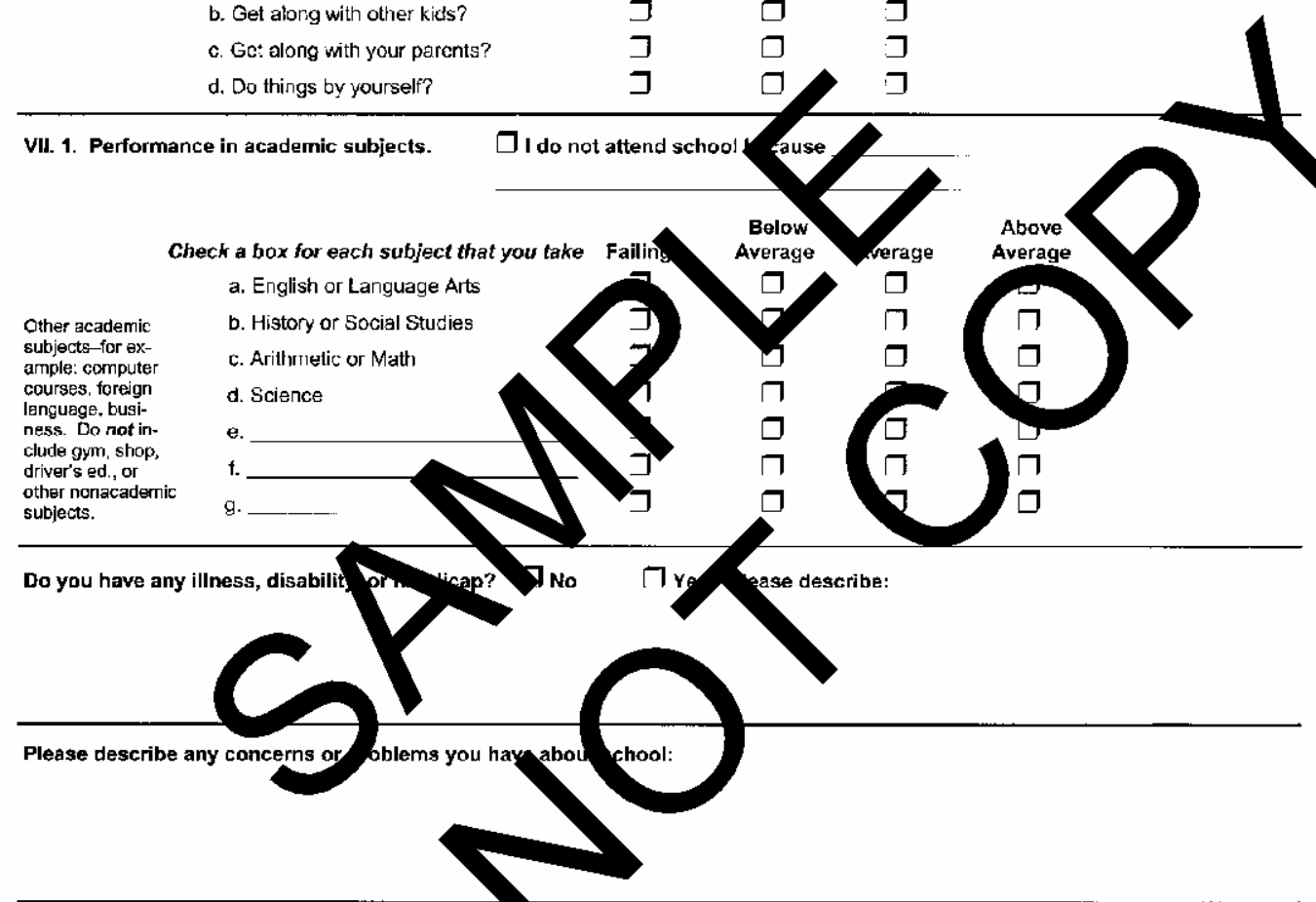

Please describe any other concerns you have:

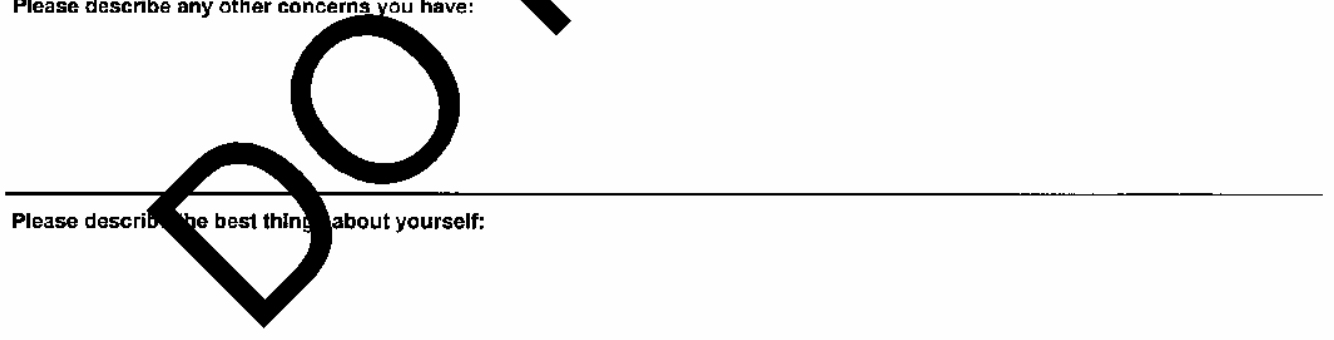


Please print. Be surc to answer all hems.

Below is a list of items that describe kids. For each item that describes you now or within the past 6 months, please circle the 2 if the item is very true or often true of you. Circle the 1 if the item is somewhat or sometimes true of you. If the item is not true of you, circle the $\boldsymbol{\theta}$.

$0=$ Not True

1 = Somewhat or Sometimes True 2 = Very True or Often True

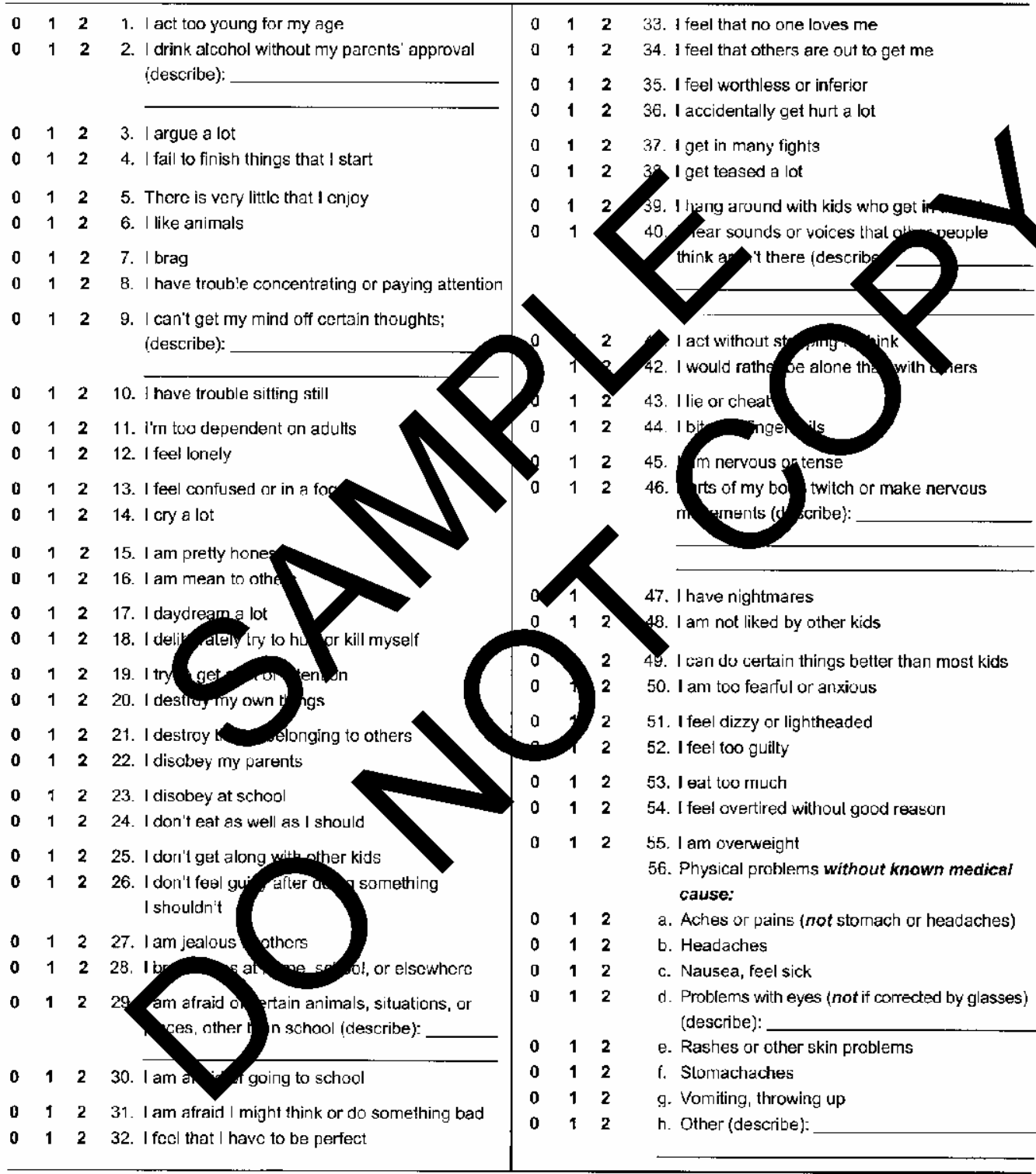


Please print. Be sure to answer all items.

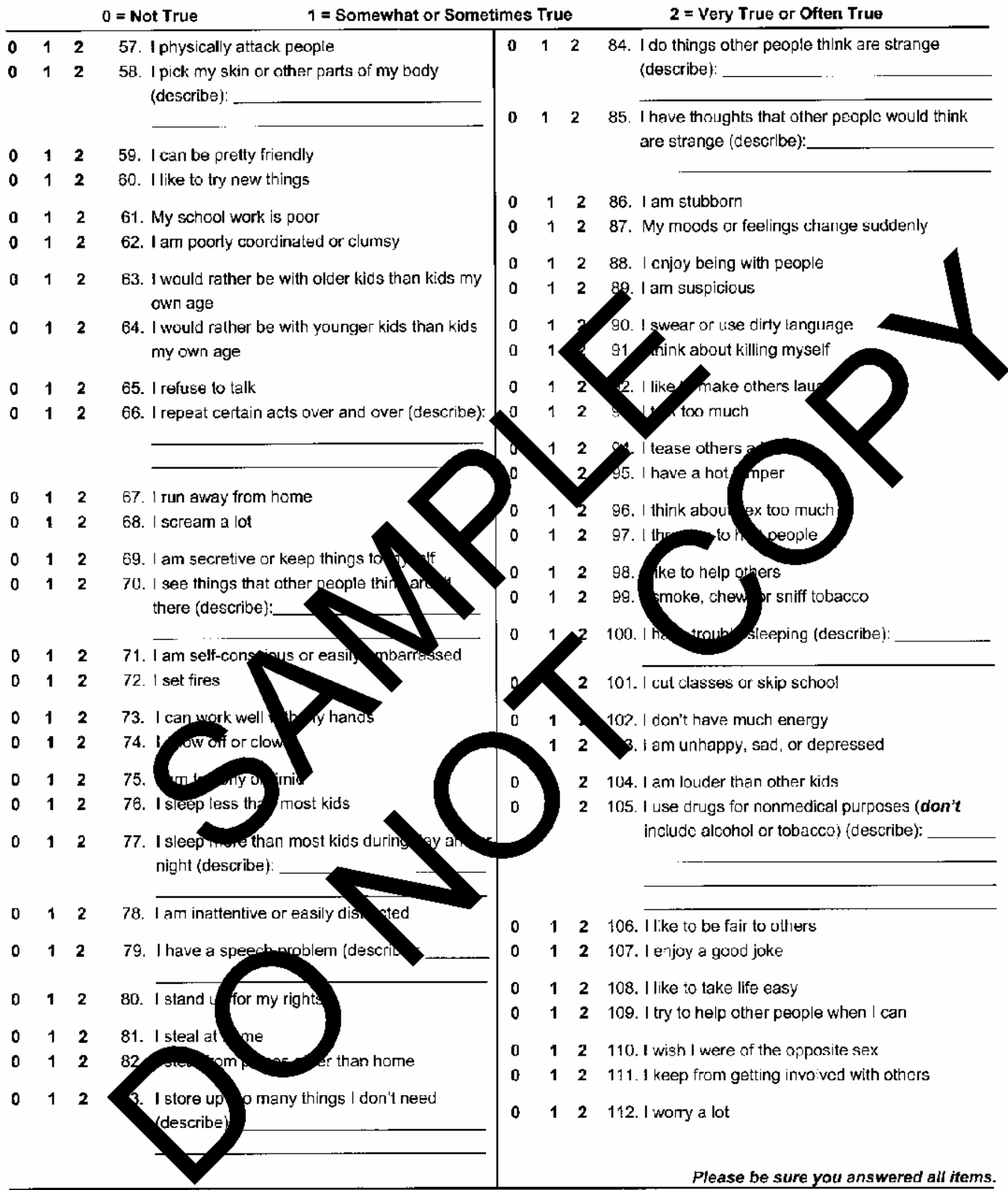

Please write down anything else that describes your feelings, behavior, or interests: 


\section{Appendix B}

\section{Sample Copy of Child Behavior Checklist}

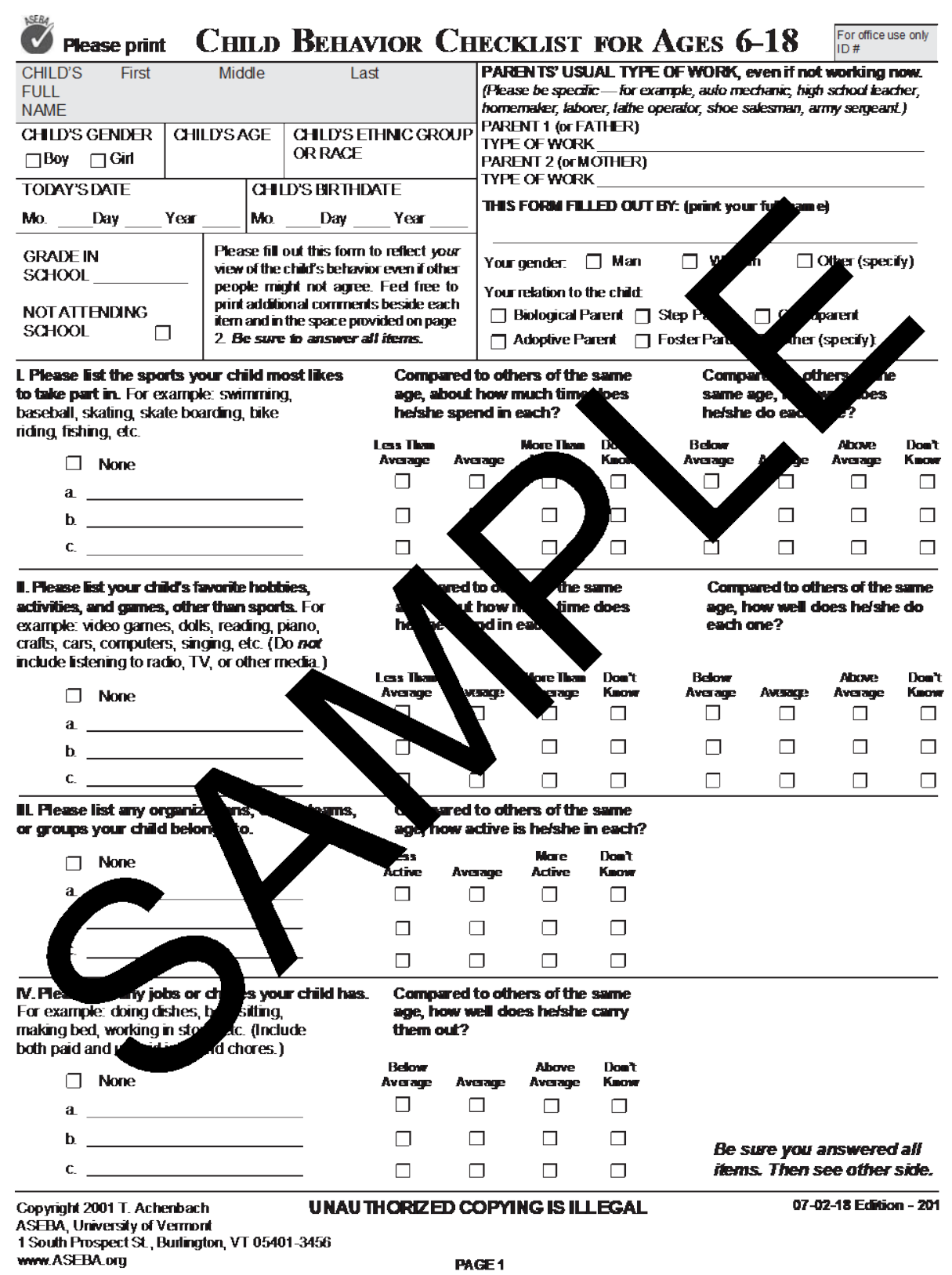


Please print. Be sure to answer all items.

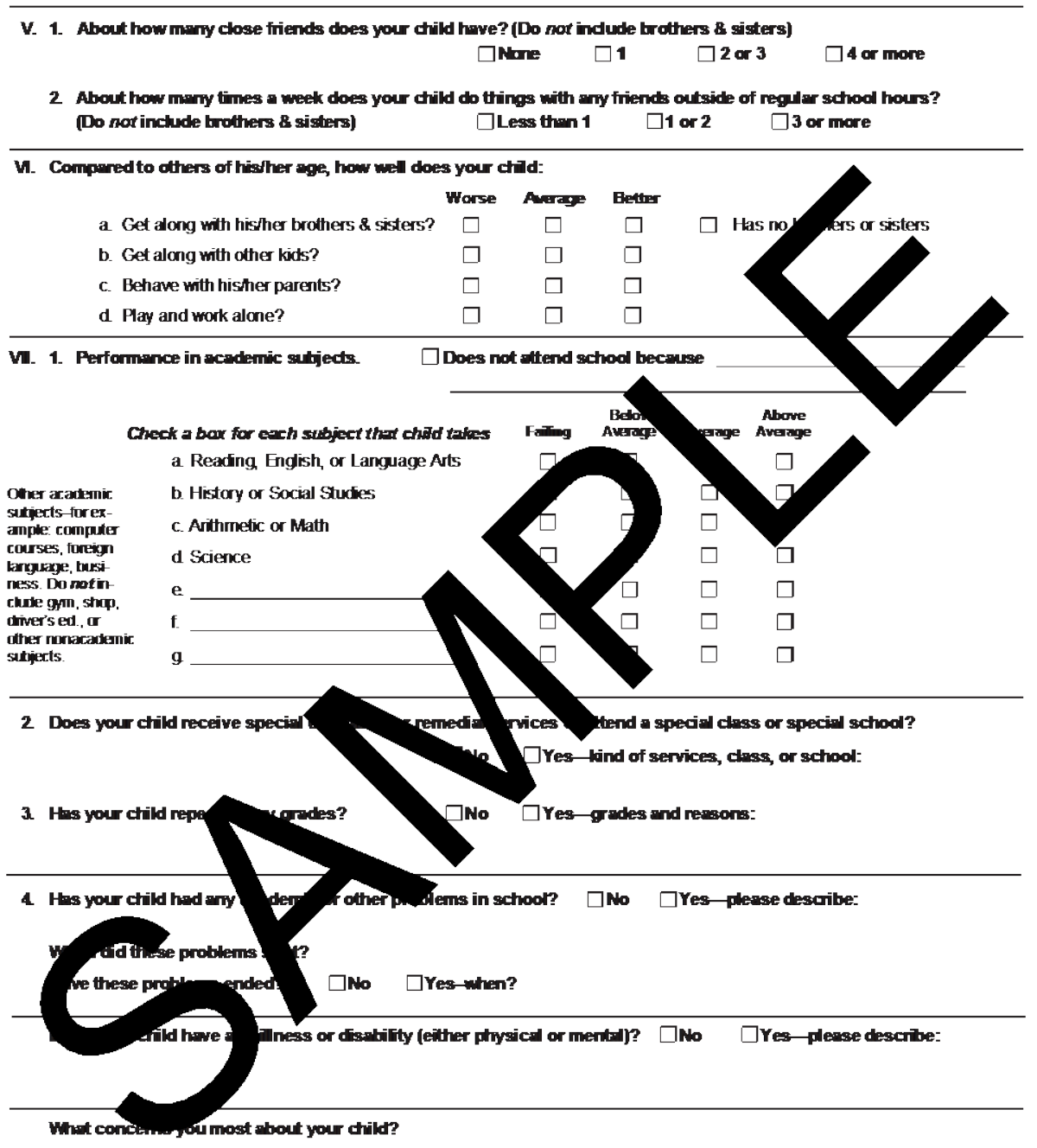

Please describe the best things about your child. 
Please print. Be sure to answer all iterns.

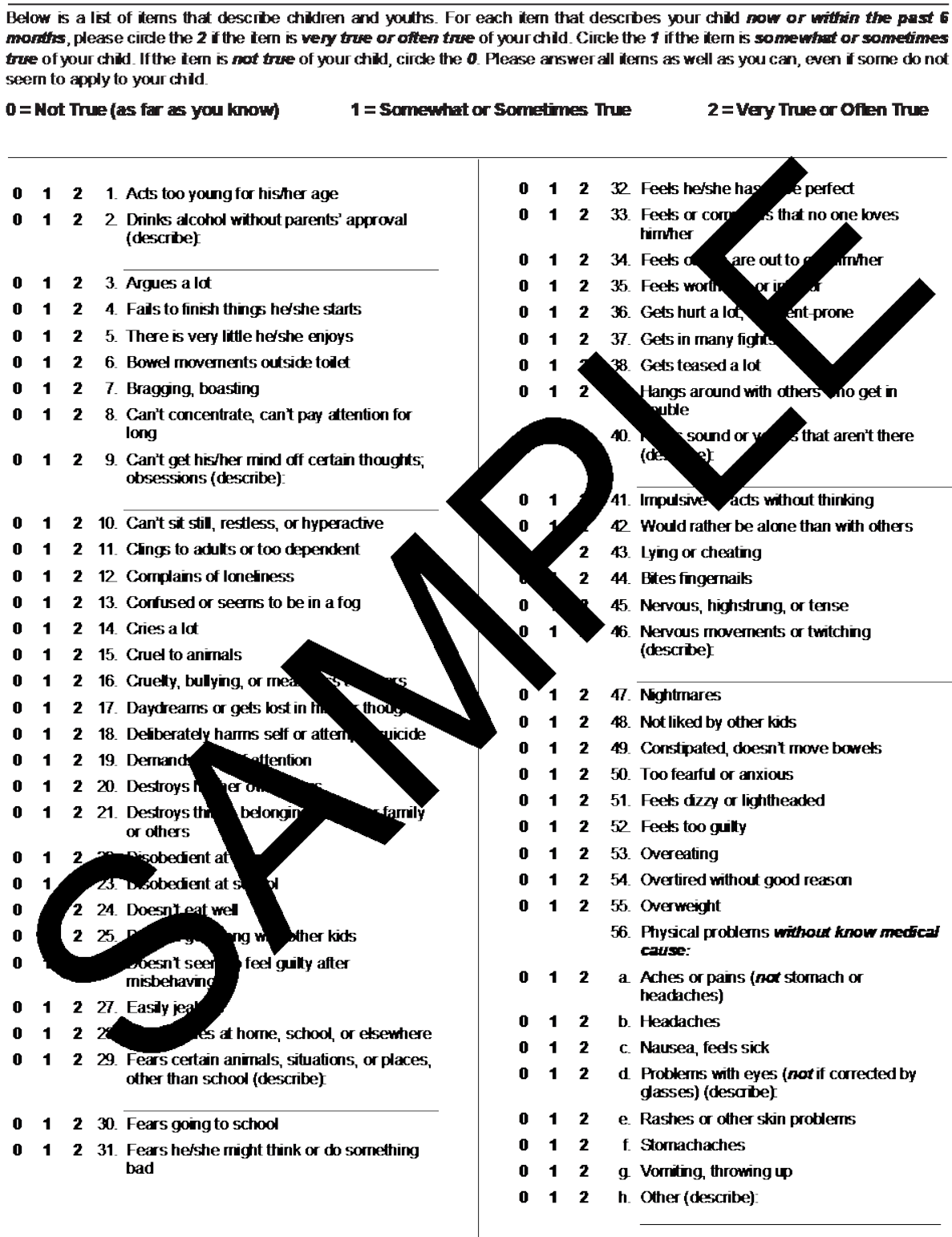

PAGE 3 Be sure you answered all items Then see other side. 
Please print. Be sure to answer all items.

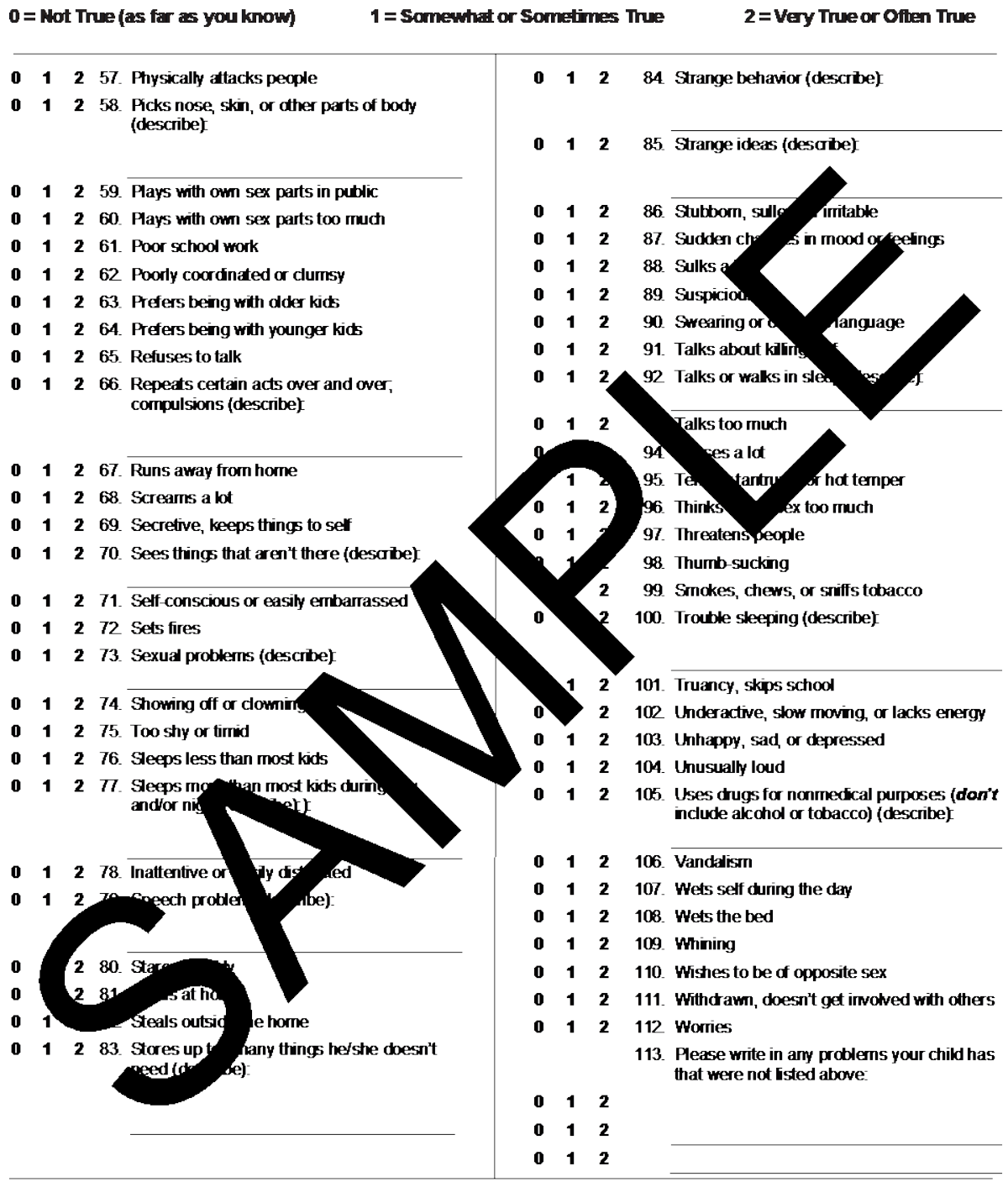




\section{Appendix C}

\section{Summary Sheet}

Participant ID\#

Adolescent Participant Summary Sheet

Location of data collection:

$\square$ САMH

$\square \mathbf{R O H}$

Current age of participant:

Age of participant at time of offence:

Month/year of index offence arrest:

Month/year of study:

Section 34 assessment:

$\square$ YES $\quad \square$ No

Mental Health Court referral

Timing of assessment:

$\square$ PRE-FINDING

$\square$ PRE-SENIENCING

$\square$ CONDITIONAL RELEASE

Index offence(s):

Conviction details:

\section{$\square$ PLEAD GUILTY}

$\square$ CONVICTED

Offence involves a co-accused:

$\square$ YES

$\square$ NO

Offence type:

$$
\begin{aligned}
& \square \text { VIOLENT ONIY } \\
& \square \text { VIOLENT AND SEXUAL } \\
& \square \text { SEXUAL ONLY }
\end{aligned}
$$

Victim type:

$\square$ UNRELATED FEMAIE
$\square$ UNRELATED MALE
$\square$ BIOLOGICALIY RELATED FEMALE
$\square$ BIOLOGICALIY RELATED MALE
$\square$ MULTIPLE UNRELATED VICTIMS
$\square$ MULTIPLE RELATED VICTIMS

\begin{tabular}{|l|}
\hline Additional Victim Detaik \\
\hline
\end{tabular}

Meets sibling incest criteria:

$\square$ YES

$\square$ NO

Parent attended appointment:

$\square$ YES

$\square$ No

Parent participated:

$\square$ YES

$\square$ NO

Researcher Initial: 
Adolescent Participant Summary Sheet

Participant ID\#

STEP 1 = Provide participant ID

Location of data collection:
$\begin{aligned} & \text { Current age of participant: } \\ & \text { STEP } 2 \text { = start with age and then move to charges } \\ & \text { Age of participant at time of offence: }\end{aligned}$ M CAMH
Month/year of index offence arrest:
Month/year of study: $\begin{array}{ll}\text { STEP } 3=\text { after establishing the charges that led to } \\ \text { the Sec. 34 referral ask if they have had any prior } \\ \text { crim. justice system involvement. }\end{array}$
Section 34 assessment:
If YES = get the type of charge + month/year + age of
participant + victim details type of previous offences.
INCLUDES charges that were dropped.

Offence involves a co-accused: $\quad \square$ YES $\quad \square$ NO $\longrightarrow$ If YES $=$ note age + gender + relationship

$\begin{array}{ll}\text { Offence type: } \quad & \square \text { VIOLENT ONLY } \\ & \square \text { VIOLENT AND SEXUAL } \\ & \square \text { SEXUAL ONLY }\end{array}$

Victim type: $\quad \square$ UNRELATED FEMALE

$\square$ UNRELATED MALE

$\square$ BIOLOGICALLY RELATED FEMALE

$\square$ BIOLOGICALLY RELATED MALE

$\square$ MULTIPLE UNRELATED VICTIMS

$\square$ MULTIPLE RELATED VICTIMS

Additional Victim Details
Note age + gender + relationship of each victim for
EVERY offence
Victim details for each previous offence can be
anywhere as long as they are labelled

\begin{tabular}{llll} 
Meets sibling incest criteria: & $\square$ YES & $\square$ NO \\
\hline Parent attended appointment: & $\square$ YES & $\square$ NO & $\square$ NOS \\
\hline Parent participated: & Researcher Initial:
\end{tabular}

If you think the parent will participate at a later time, leave this part blank until it is confirmed either way 


\section{Appendix D}

\section{Summary Sheet Coding Form}

Subject: Participant number

\section{Participation_Location:}

1- $\mathrm{CAMH}$

2- $\mathrm{ROH}$

Parent type: (String variable; for parent summary sheet data only)

Participation_Age: Age at time of participation (continuous variable)

Summary_Sheet_Informant: Who is providing the data on the summary sheet?

1- Adolescent

2- Parent/guardian

Index_Offence_Age: Age when index offence occurred (continuous variable)

Index_Offence_Age_Charged: Age when charged for index offence (if different than age when offence occurred; continuous variable)

Index_Offence_Month_of_Arrest: Month/date when arrested for index offence

Month_of_Participation: Month/date of participation

Months_Between_Index_Arrest_and_Participation: How many months in between the arrest for index offence and date of participation in study

Index_Offence_Historical: Was the participant charged for their index offence more than one year after the offence occurred?

\section{Section_34_Assessment:}

1- Yes

0 - No

\section{Mental_Health_Court:}

1- Yes

0 - No 
Mental_Health_Diagnoses: Any diagnoses that have been given and are reason for index offence mental health court deferral (as written on summary sheet)

Timing of Assessment: When is the assessment taking place? (Index offence only)

1- $\quad$ Pre-finding assessment (if pre-finding report, plead is not applicable)

2- $\quad$ Pre-sentencing assessment

3- Conditional release assessment

4- Other assessment timing

Index_Offence_Plead: Of index offence (if pre-finding report, plead not applicable)

1- Plead guilty

2- Convicted (tried and found guilty; or if found guilty but currently appealing)

Index Offence: String variable of offence (what was written on summary sheet)

Index_Offence_Type:

1- Violent only

2- Violent and sexual

3- Sexual only

Index_Offence_Sibling_Incest: (Only if biologically related female or an unrelated female is victim of index offence)

1- Yes

0 - No

Subsequent_Cahrge_After_Index: Has the participant been charged with anything since index offence?

1- Yes

0 - No

Subsequent_Charges: String variable of subsequent charges

Index_Offence_Breach: Was there a breach of conditions in relation to index offence?

1- Yes

0 - No

\section{Index_Offence_Breach_Type:}

1- Breach of bail conditions 
2- Breach of probation conditions

Index_Offence_Occur_Inside_Home: Did the index offence occur inside the offender's home? (If offender is living in a group home, the group home is not regarded to be their home).

1- Yes

0 - No

Parent of Sex Offender: (Only on parent summary sheet database)

1- Yes

0 - No

Index_Offence_Involves_Co-Accused:

1- Yes

0 - No

Index_Offence_Older_Co-Accused: Does the index offence involve a co-accused who is older than the participant?

1- Yes

0 - No

Index_Offence_Co-Accused_Age_Category: What age category does co-accused 1 fall into at the time of the index offence?

1- Child (0 to 12 years)

2- Younger teen (younger than age of offender at time of offence)

3- Same age teen (same age as offender at time of offence)

4- Older teen (older than offender at time of offence)

5- Young adult (18-25 years)

6- Adult (over 25 years)

Index_Offence_Co-Accused_1_Age: Age of co-accused 1 at time of index offence

Co-Accused_1_Sex: Sex of co-accused 1 involved in index offence

1- Female

2- Male 
Co-Accused_1_Relation: Relationship of co-accused 1 involved in index offence

1- Biological sibling

2- Non-biological sibling

3- Cousin

4- Friend

5- Romantic partner (of either sex)

6- Group home peer

7- Same-age acquaintance

8- Older acquaintance

9- Parent (biological, adoptive or foster)

Repeat co-accused details for co-accused 2-3 involved in index offence if applicable.

Index_Multiple_Victims: Were there multiple victims involved in the index offence?

1- Yes

0 - No

Index_Number_of_Victims: How many victims were involved in index offence?

Index_Victim_1_Age_Category: What age category does victim 1 fall into at the time of the index offence?

1- Child (0 to 12 years)

2- Younger teen (younger than age of offender at time of offence)

3- Same age teen (same age as offender at time of offence)

4- Older teen (older than offender at time of offence)

5- Young adult (18-25 years)

6- Adult (over 25 years)

Index_Victim_1_Age: Victim 1 age at time of the index offence (continuous variable)

\section{Index_Victim_1_Sex:}

1- Female

2- Male 
Index_Victim_1_Relation: Relation of victim 1 involved in index offence

1- Biological sibling

2- Non-biological sibling

3- Friend

4- Romantic partner (of either sex)

5- Group home peer

6- Same-age acquaintance

7- Older acquaintance

8- Worker (includes group home worker, social worker, and probation officer)

9- School staff

10- Parent (biological, adoptive, or foster)

11- Property owner

12- Stranger

13- Victim online only

Repeat details for victims 2-5 of index offence if applicable.

Previous_Charges: Does the participant have previous charges?

$1-$ Yes

$0-$ No

Previous_Incident_Charges: What was the participant charged with at previous offence? (What was written on summary sheet; string variable)

*Previous offence labelled "incident 1" should be the participant's most recent charge after index offence "incident 2 " is second most recent, and so on

Previous_Incident_1_Month: Month that the participant was charged for previous incident 1 (string variable)

Incident_1_Age: Participant age at time of previous offence 1 (continuous variable)

Incident_1_Involve_Breach: Does the previous incident 1 involve a breach of conditions for incident 1 charges?

$1-$ Yes

0 - No

\section{Incident_1_Breach_Type:}

1- Breach of bail conditions

2- Breach of probation conditions 
Incident_1_Involve_Co-Accused: Did the previous incident 1 involve a co-accused?

1- Yes

0 - No

Incident_1_Older_Co-Accused: Did the previous incident 1 involve a co-accused who was older than the participant at the time of offence?

1- Yes

0 - No

Incident_1_Co-Accused_1_Age_Category: What age category does the co-accused 1 involved in previous incident 1 fall into?

1- Child (0 to 12 years)

2- Younger teen (younger than age of offender at time of offence)

3- Same age teen (same age as offender at time of offence)

4- Older teen (older than offender at time of offence)

5- Young adult (18-25 years)

6- Adult (over 25 years)

Incident_1_Co-Accused_1_Age: Age of co-accused 1 at time of previous incident 1 (continuous variable)

Incident_1_Co-Accused_1_Sex: Sex of co-accused 1 involved in previous incident 1

1- Female

2- Male

Incident_1_Co-Accused_1_Relation: Relationship of co-accused 1 involved in previous incident $\overline{1}$

1- Biological sibling

2- Non-biological sibling

3- Cousin

4- Friend

5- Romantic partner (of either sex)

6- Group home peer

7- Same-age acquaintance

8- Older acquaintance

9- Parent (biological, adoptive or foster)

Incident_1_Multiple_Victims: Does previous incident 1 include multiple victims? 
$1-$ Yes

$0-\mathrm{No}$

Incident_1_Victim_1_Age_Category: What age category does victim 1 fall into at the time of previous incident 1 ?

0 - Child (0 to 12 years)

1- Younger teen (younger than age of offender at time of offence)

2- Same age teen (same age as offender at time of offence)

3- Older teen (older than offender at time of offence)

4- Young adult (18-25 years)

5- Adult (over 25 years)

Incident_1_Previous_Victim_1_Age: Victim 1 age at time of previous incident 1

Incident_1_Previous_Victim_1_Sex: Sex of victim 1 involved in previous incident 1

1- Female

2- Male

Incident_1_Previous_Victim_1_Relation: Relationship of victim 1 involved in previous incident 1

1- Biological sibling

2- Non-biological sibling

3- Friend

4- Romantic partner (of either sex)

5- Group home peer

6- Same-age acquaintance

7- Older acquaintance

8- Worker (includes group home worker, social worker, and probation officer)

9- School staff

10- Parent (biological, adoptive, or foster)

11- Property owner

12- Stranger

13-Victim online only

Repeat details for previous incidents 2-3 if applicable, and for victim 2 of previous incidents if applicable.

\section{Parent/Adolescent_Participation:}

$1-$ Yes

0 - No 
Parent_Not_Attending_Reason: If parent did not attend adolescent's appointment, what was the reason given for not attending? (String variable).

Total_Time_in_CJS: The total amount of time participant has spent in criminal justice system, calculated in years between the date of their first ever charge and the date of participation in study. If less than a year, use a decimal and round to the nearest quarter. 


\section{Appendix E}

\section{Background Survey}

\section{$\underline{\text { Demographic Information }}$}

1. How old are you today?
a) 12
b) 13
c) 14
d) 15
e) 16
f) 17
g) 18

2. What is your ethnicity?
a) Caucasian/White
b) African-Canadian/Caribbean/Black
c) Asian-Canadian
d) Aboriginal/Métis
e) Latino
f) Other, specify:

3. What grade are you in at school?

\section{Index Offence Details}

4. What type of offense have you been charged with?
a) Sexual offense
b) Violent and sexual offense
c) Violent only offense
d) Drug offense

5. Is this the first time you have been charged with this type of offense?
a) Yes
b) $\mathrm{No}$

6. How old were you when you committed the offense you have been charged with? 
7. How old were you when you were officially charged for the offense you have been charged with?

8. How many victims were involved in your offense?

9. What is the gender of the victim involved in your offense?

a) Male

b) Female

10. How old was the victim involved in your offense?

11. Which best describes the relationship you have with the victim in your offense?

Select all that apply.

a) Biological sibling (includes half-siblings)

b) Non-biological siblings (includes adopted, foster, or step-siblings)

c) Cousin

d) Friend from the neighborhood

e) Someone you know from the neighborhood

f) Friend from school

g) Someone you know from school

h) Stranger

12. Was the victim involved in your offense living with you at the time the offense happened?

a) Yes

b) No

13. Did you physically hurt the victim involved in your offense?

a) Yes

b) No

14. How badly was the victim involved in your offense physically hurt?

Select all that apply.

a) Cuts and bruises

b) Bleeding

c) Broken bones

d) Unconscious

e) They went to the hospital 
f) An ambulance had to come

g) The victim did not get hurt

15. Did you feel out of control when you committed the offense you have been charged with?
a) Yes
b) $\mathrm{No}$

16. Were you under the influence of alcohol (that is, were you drunk) during your offense?
a) Yes
b) No

17. Were you under the influence of drugs (that is, were you high) during your offense?
a) Yes
b) No

\section{Family Background}

The next set of questions have to do with the environment you grew up in

18. Did your parents live in the same house?
a) Yes
b) No

19. Did your biological mother live in the same home as you?
a) Yes
b) No

20. Did your biological father live in the same home as you?
a) Yes
b) No

21. How many siblings did you have living with you? 
22. What type of siblings did you have living with you?

\section{Select all that apply.}

a) Older biological sister

b) Older biological brother

c) Older non-biological sister (adopted/foster/step)

d) Older non-biological brother (adopted/foster/step)

e) Younger biological sister

f) Younger biological brother

g) Younger non-biological sister (adopted/foster/step)

h) Younger non-biological brother (adopted/foster/step)

i) I do not have siblings

\section{Where are you currently living?}
a) At home
b) At a friend's house
c) With other family members
d) In a group home
e) With a foster family
f) In a correctional facility
g) Alone
h) Other

24. Were you ever removed from your home because it was unsafe?
a) Yes
b) No

25. BEFORE your offense, how often did Children's Aid come to your home?
a) Once or twice
b) A couple time's a year
c) Once a month
d) A couple time's a month
e) Once a week
f) Every day
g) Never

26. BEFORE your offense, how often did the police come to your home?
a) Once or twice
b) A couple time's a year
c) Once a month
d) A couple time's a month 

e) Once a week
f) Every day
g) Never

27. How often were the adults at home violent with each other?
a) Once or twice
b) A couple times a year
c) Once a month
d) A couple times a month
e) Once a week
f) Every day
g) Never

28. How often were the adults at home verbally abusive with each other?
a) Once or twice
b) A couple time's a year
c) Once a month
d) A couple time's a month
e) Once a week
f) Every day
g) Never

29. How often did the adults at home drink alcohol?
a) Once or twice
b) A couple times a year
c) Once a month
d) A couple times a month
e) Once a week
f) Every day
g) Never

30. How often did the adults at home do illegal drugs? (for example, smoke marijuana)
a) Once or twice
b) A couple times a year
c) Once a month
d) A couple times a month
e) Once a week
f) Every day
g) Never 
31. How often did the adults at home misuse prescription medication? (for example, pain medication to get high)
a) Once or twice
b) A couple times a year
c) Once a month
d) A couple times a month
e) Once a week
f) Every day
g) Never

32. How often were you uncomfortable going home?
a) Once or twice
b) A couple times a year
c) Once a month
d) A couple times a month
e) Once a week
f) Every day
g) Never - I was always comfortable going home

33. How often did you avoid going home?
a) Once or twice
b) A couple times a year
c) Once a month
d) A couple times a month
e) Once a week
f) Every day
g) Never - I did not avoid going home

\section{Maltreatment History}

34. How often did the adults at home physically abuse you?
a) Once or twice
b) A couple times a year
c) Once a month
d) A couple times a month
e) Once a week
f) Every day
g) Never - the adults were not physically abusive 
35. Who at home physically hurt you?

\section{Select all that apply.}
a) Biological mother
b) Non-biological mother
c) Biological father
d) Non-biological father
e) Grandparents
f) Other female relative (aunt)
g) Other male relative (uncle)
h) Female family friends
i) Male family friends
j) No one

36. How often did the adults at home physically abuse any of your siblings?

Press 0 if you do not have siblings.
a) Once or twice
b) A couple times a year
c) Once a month
d) A couple times a month
e) Once a week
f) Every day
g) Never

37. Who at home physically abused your siblings?

Press 0 if you do not have siblings.
a) Biological mother
b) Non-biological mother
c) Biological father
d) Non-biological father
e) Grandparents
f) Other female relative (aunt)
g) Other male relative (uncle)
h) Female family friends
i) Male family friends
j) No one 
38. How often did the adults at home sexually abuse you?
a) Once or twice
b) A couple times a year
c) Once a month
d) A couple times a month
e) Once a week
f) Every day
g) Never

39. Who at home sexually abused you?

Select all that apply.
a) Biological mother
b) Non-biological mother
c) Biological father
d) Non-biological father
e) Grandparents
f) Other female relative (aunt)
g) Other male relative (uncle)
h) Female family friends
i) Male family friends
j) No one

40. How often did the adults at home sexually abuse any of your siblings?

Press 0 if you do not have siblings.
a) Once or twice
b) A couple times a year
c) Once a month
d) A couple times a month
e) Once a week
f) Every day
g) Never

41. Who at home sexually abused your siblings?

Press 0 if you do not have siblings.
a) Biological mother
b) Non-biological mother
c) Biological father
d) Non-biological father
e) Grandparents 
f) Other female relative (aunt)

g) Other male relative (uncle)

h) Female family friends

i) Male family friends

j) No one

42. How often did the adults at home verbally abuse you?
a) Once or twice
b) A couple times a year
c) Once a month
d) A couple times a month
e) Once a week
f) Every day
g) Never - the adults were not physically abusive

\section{Who at home verbally abused you?}

\section{Select all that apply.}
a) Biological mother
b) Non-biological mother
c) Biological father
d) Non-biological father
e) Grandparents
f) Other female relative (aunt)
g) Other male relative (uncle)
h) Female family friends
i) Male family friends
j) No one

44. How often did the adults at home verbally abuse any of your siblings?

\section{Press 0 if you do not have siblings.}
a) Once or twice
b) A couple times a year
c) Once a month
d) A couple times a month
e) Once a week
f) Every day
g) Never 
45. Who at home verbally abused your siblings?

Press 0 if you do not have siblings.
a) Biological mother
b) Non-biological mother
c) Biological father
d) Non-biological father
e) Grandparents
f) Other female relative (aunt)
g) Other male relative (uncle)
h) Female family friends
i) Male family friends
j) No one

46. How often did the adults at home emotionally abuse you?
a) Once or twice
b) A couple times a year
c) Once a month
d) A couple times a month
e) Once a week
f) Every day
g) Never - the adults were not physically abusive

47. Who at home emotionally abused you?

Select all that apply.
a) Biological mother
b) Non-biological mother
c) Biological father
d) Non-biological father
e) Grandparents
f) Other female relative (aunt)
g) Other male relative (uncle)
h) Female family friends
i) Male family friends
j) No one 
48. How often did the adults at home emotionally abuse any of your siblings?

Press 0 if you do not have siblings.
a) Once or twice
b) A couple times a year
c) Once a month
d) A couple times a month
e) Once a week
f) Every day
g) Never

49. Who at home emotionally abused your siblings?

Press 0 if you do not have siblings.
a) Biological mother
b) Non-biological mother
c) Biological father
d) Non-biological father
e) Grandparents
f) Other female relative (aunt)
g) Other male relative (uncle)
h) Female family friends
i) Male family friends
j) No one

50. How often did the adults at home neglect you?
a) Once or twice
b) A couple times a year
c) Once a month
d) A couple times a month
e) Once a week
f) Every day
g) Never

51. Who at home neglected you?

Select all that apply.
a) Biological mother
b) Non-biological mother
c) Biological father
d) Non-biological father
e) Grandparents 
f) Other female relative (aunt)

g) Other male relative (uncle)

h) Female family friends

i) Male family friends

j) No one

52. How often did the adults at home neglect any of your siblings?

Press 0 if you do not have siblings.
a) Once or twice
b) A couple times a year
c) Once a month
d) A couple times a month
e) Once a week
f) Every day
g) Never

53. Who at home neglected your siblings?

Press 0 if you do not have siblings.
a) Biological mother
b) Non-biological mother
c) Biological father
d) Non-biological father
e) Grandparents
f) Other female relative (aunt)
g) Other male relative (uncle)
h) Female family friends
i) Male family friends
j) No one 


\section{Appendix F}

Modified Revised Child and Adolescent Depression Scale - Short Version Ever since I was charged I feel sad or empty

$\begin{array}{cccc}1 & 2 & 3 & 4 \\ \text { Never } & \text { Sometimes } & \text { Often } & \text { Always }\end{array}$

Did you feel sad or empty before you were charged?
1
Sometimes
3
4
Never
Often
Always

Sometimes

Ever since I was charged, I worry when I think I have not done well at something

$\begin{array}{cccc}1 & 2 & 3 & 4 \\ \text { Never } & \text { Sometimes } & \text { Often } & \text { Always }\end{array}$

Did you worry about not doing well before you were charged?

$\begin{array}{cccc}1 & 2 & 3 & 4 \\ \text { Never } & \text { Sometimes } & \text { Often } & \text { Always }\end{array}$

Ever since I was charged, I feel afraid of being home alone

$\begin{array}{cccc}1 & 2 & 3 & 4 \\ \text { Never } & \text { Sometimes } & \text { Often } & \text { Always }\end{array}$

Were you afraid of being home alone before you were charged?
1
Never
2
Sometimes
3
Often

Ever since I was charged, nothing is fun anymore

$\begin{array}{cccc}1 & 2 & 3 & 4 \\ \text { Never } & \text { Sometimes } & \text { Often } & \text { Always }\end{array}$

Did you feel like nothing was fun before you were charged?

$\begin{array}{cccc}1 & 2 & 3 & 4 \\ \text { Never } & \text { Sometimes } & \text { Often } & \text { Always }\end{array}$


Ever since I was charged, I feel worried that something terrible will happen to the people I care about

$\begin{array}{cccc}1 & 2 & 3 & 4 \\ \text { Never } & \text { Sometimes } & \text { Often } & \text { Always }\end{array}$

Did you worry about something terrible happening before you were charged?

$\begin{array}{cccc}1 & 2 & 3 & 4 \\ \text { Never } & \text { Sometimes } & \text { Often } & \text { Always }\end{array}$

Ever since I was charged, I am afraid of being in crowded places (for example, the mall or a bus)

$\begin{array}{cccc}1 & 2 & 3 & 4 \\ \text { Never } & \text { Sometimes } & \text { Often } & \text { Always }\end{array}$

Were you afraid of being in crowded places before you were charged?
1
Never
2
Sometimes
3
Often
4
Always

Ever since I was charged, I worry about what other people think of me
1
2
Sometimes
3
Often
4
Always

Did you worry about what other people thought of you before you were charged?

$\begin{array}{cccc}1 & 2 & 3 & 4 \\ \text { Never } & \text { Sometimes } & \text { Often } & \text { Always }\end{array}$

Ever since I was charged, I have trouble sleeping

$\begin{array}{cccc}1 & 2 & 3 & 4 \\ \text { Never } & \text { Sometimes } & \text { Often } & \text { Always }\end{array}$

Did you have trouble sleeping before you were charged?
1
Never
2
Sometimes
3
Often
Always

Ever since I was charged, I feel scared if I have to sleep by myself
1
2
Sometimes
3
Often 
Were you scared to sleep by yourself before you were charged?

$\begin{array}{cccc}1 & 2 & 3 & 4 \\ \text { Never } & \text { Sometimes } & \text { Often } & \text { Always }\end{array}$

Ever since I was charged, I have trouble with my appetite

$\begin{array}{cccc}1 & 2 & 3 & 4 \\ \text { Never } & \text { Sometimes } & \text { Often } & \text { Always }\end{array}$

Did you have trouble with your appetite before you were charged?

$\begin{array}{cccc}1 & 2 & 3 & 4 \\ \text { Never } & \text { Sometimes } & \text { Often } & \text { Always }\end{array}$

Ever since I was charged, I become dizzy or I faint for no reason
1
Never
Sometimes
3

Did you become dizzy or faint for no reason before you were charged?

$\begin{array}{cccc}1 & 2 & 3 & 4 \\ \text { Never } & \text { Sometimes } & \text { Often } & \text { Always }\end{array}$

Ever since I was charged, I have to do somethings over and over (for example, washing my hands)

$\begin{array}{cccc}1 & 2 & 3 & 4 \\ \text { Never } & \text { Sometimes } & \text { Often } & \text { Always }\end{array}$

Did you have to do some things over and over before you were charged?

$\begin{array}{cccc}1 & 2 & 3 & 4 \\ \text { Never } & \text { Sometimes } & \text { Often } & \text { Always }\end{array}$

Ever since I was charged, I do not have any energy
1
Never
Sometimes
3

Did you feel like you had energy before you were charged?

$\begin{array}{cccc}1 & 2 & 3 & 4 \\ \text { Never } & \text { Sometimes } & \text { Often } & \text { Always }\end{array}$


Ever since I was charged, I tremble or shake for no reason

$\begin{array}{cccc}1 & 2 & 3 & 4 \\ \text { Never } & \text { Sometimes } & \text { Often } & \text { Always }\end{array}$

Did you tremble or shake for no reason before you were charged?

$\begin{array}{cccc}1 & 2 & 3 & 4 \\ \text { Never } & \text { Sometimes } & \text { Often } & \text { Always }\end{array}$

Did you feel like you had energy before you were charged?

$\begin{array}{cccc}1 & 2 & 3 & 4 \\ \text { Never } & \text { Sometimes } & \text { Often } & \text { Always }\end{array}$

Ever since I was charged, I cannot think clearly
1
Never
Sometimes
3
4
Always

Could you think clearly before you were charged?

$\begin{array}{cccc}1 & 2 & 3 & 4 \\ \text { Never } & \text { Sometimes } & \text { Often } & \text { Always }\end{array}$

Ever since I was charged, I feel worthless

$\begin{array}{cccc}1 & 2 & 3 & 4 \\ \text { Never } & \text { Sometimes } & \text { Often } & \text { Always }\end{array}$

Did you feel worthless before you were charged?

$\begin{array}{cccc}1 & 2 & 3 & 4 \\ \text { Never } & \text { Sometimes } & \text { Often } & \text { Always }\end{array}$

Ever since I was charged, I have to think special thoughts to stop bad things from happening (for example, counting)

$\begin{array}{cccc}1 & 2 & 3 & 4 \\ \text { Never } & \text { Sometimes } & \text { Often } & \text { Always }\end{array}$

Did you have to think special thoughts before you were charged?
1
2
Sometimes
3
Often
Never
Always 
Ever since I was charged, I think about death

$\begin{array}{cccc}1 & 2 & 3 & 4 \\ \text { Never } & \text { Sometimes } & \text { Often } & \text { Always }\end{array}$

Did you think about death before you were charged?

$\begin{array}{cccc}1 & 2 & 3 & 4 \\ \text { Never } & \text { Sometimes } & \text { Often } & \text { Always }\end{array}$

Ever since I was charged, I feel like I do not want to move

$\begin{array}{cccc}1 & 2 & 3 & 4 \\ \text { Never } & \text { Sometimes } & \text { Often } & \text { Always }\end{array}$

Did you feel like you wanted to move before you were charged?
1
Never
Sometimes
3

Ever since I was charged, I worry I will suddenly start feeling scared, even when there is nothing to be afraid of
1
Never
2
Sometimes
3
4
Always

Did you worry about suddenly feeling scared before you were charged?

$\begin{array}{cccc}1 & 2 & 3 & 4 \\ \text { Never } & \text { Sometimes } & \text { Often } & \text { Always }\end{array}$

Ever since I was charged, I feel tired a lot

$\begin{array}{cccc}1 & 2 & 3 & 4 \\ \text { Never } & \text { Sometimes } & \text { Often } & \text { Always }\end{array}$

Were you tired a lot before you were charged?
1
Never
Sometimes
3
Often
4
Always

Ever since I was charged, I feel afraid that I will make a fool out of myself in front of people

$\begin{array}{cccc}1 & 2 & 3 & 4 \\ \text { Never } & \text { Sometimes } & \text { Often } & \text { Always }\end{array}$


Were you afraid of making a fool out of yourself before you were charged?

$\begin{array}{cccc}1 & 2 & 3 & 4 \\ \text { Never } & \text { Sometimes } & \text { Often } & \text { Always }\end{array}$

Ever since I was charged, I have to do things in just the right way to stop bad things from happening

$\begin{array}{cccc}1 & 2 & 3 & 4 \\ \text { Never } & \text { Sometimes } & \text { Often } & \text { Always }\end{array}$

Did you have to do things in just the right way before you were charged?

$\begin{array}{cccc}1 & 2 & 3 & 4 \\ \text { Never } & \text { Sometimes } & \text { Often } & \text { Always }\end{array}$

Ever since I was charged, I feel restless
1
Never
$\stackrel{2}{\text { Sometimes }}$
3
4
Often
Always

Did you feel restless before you were charged?
1
2
3
4
Never
Sometimes
Often
Always

Ever since I was charged, I worry that something bad will happen to me

$\begin{array}{cccc}1 & 2 & 3 & 4 \\ \text { Never } & \text { Sometimes } & \text { Often } & \text { Always }\end{array}$

Did you worry that something bad would happen to you before you were charged?

$\begin{array}{cccc}1 & 2 & 3 & 4 \\ \text { Never } & \text { Sometimes } & \text { Often } & \text { Always }\end{array}$




\section{Appendix G}

\section{Modified Rosenberg Self-Esteem Scale}

Ever since I was charged, I feel like I am worth as much as other people

$\begin{array}{cccc}1 & 2 & 3 & 4 \\ \text { Strongly Agree } & \text { Agree } & \text { Disagree } & \text { Strongly Disagree }\end{array}$

Did you feel like you had as much worth as other people before you were charged? 1

Strongly Agree

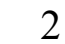

Agree
3

Disagree
4

Strongly Disagree

Ever since I was charged, I feel like I have a lot of good qualities
1
2
Agree
3
4
Strongly Agree
Disagree
Strongly Disagree

Did you feel like you had a lot of good qualities before you were charged?
1
Strongly Agree
2
Agree
Disagree
4
Strongly Disagree

Ever since I was charged, I feel like I am a failure
1
2
3
4
Strongly Agree
Agree
Disagree
Strongly Disagree

Did you feel like a failure before you were charged?
1
2
3
4
Strongly Agree
Agree
Disagree
Strongly Disagree

Ever since I was charged, I feel like I can do things as well as other people
1
Strongly Agree
2
Agree
3
Disagree
4
Strongly Disagree

Did you feel like you could do things as well as other people before you were charged?
1
Strongly Agree
2
Agree
3
Disagree
4
Strongly Disagree 
Ever since I was charged, I feel like I have nothing to be proud of
1
2
3
Disagree
4
Strongly Disagree
Strongly Agree
Agree

Did you feel like you could be proud before you were charged?
1
Strongly Agree
2
Agree
3
Disagree
4
Strongly Disagree

Ever since I was charged, I feel positive about myself
1
2
3
Strongly Agree
Agree
Disagree
4
Strongly Disagree

Did you feel positive about yourself before you were charged?
1
2
Agree
3
Disagree
4
Strongly Agree
Strongly Disagree

Agree

Ever since I was charged, I feel satisfied with myself
1
2
3
4
Strongly Agree
Agree
Disagree
Strongly Disagree

Did you feel satisfied with yourself before you were charged?
1
Strongly Agree
2
Agree
Disagree
4
Strongly Disagree

Ever since I was charged, I wish I could have more respect for myself
1
Strongly Agree
2
Agree
3
Disagree
4
Strongly Disagree

Did you wish you could have more respect for yourself before you were charged?

$\begin{array}{cccc}1 & 2 & 3 & 4 \\ \text { Strongly Agree } & \text { Agree } & \text { Disagree } & \text { Strongly Disagree }\end{array}$

Ever since I was charged, I feel useless
1
2
3
4
Strongly Agree
Agree
Disagree
Strongly Disagree 
Did you feel useless before you were charged?
1 2
3
4
Agree
Disagree
Strongly Disagree

Strongly Agree

Ever since I was charged, I feel like I am no good at all

$\begin{array}{cccc}1 & 2 & 3 & 4 \\ \text { Strongly Agree } & \text { Agree } & \text { Disagree } & \text { Strongly Disagree }\end{array}$

Did you feel like you were no good at all before you were charged?

1

Strongly Agree
2

Agree
3

Disagree
4

Strongly Disagree 


\section{Appendix H}

Modified Multidimensional Scale of Perceived Social Support for Adolescents Ever since I was charged, I have a special person around when I need them

$\begin{array}{ccccccc}1 & 2 & 3 & 4 & 5 & 6 & 7 \\ \text { Very } & \text { Strongly } & \text { Somewhat } & \text { Neutral } & \text { Somewhat } & \text { Strongly } & \text { Very } \\ \text { Strongly } & \text { Disagree } & \text { Agree } & \text { Strongly } \\ \text { Disagree } & \text { Disagree } & & & \text { Agree }\end{array}$

Was your special person around when you needed them before you were charged?

$\begin{array}{ccccccc}1 & 2 & 3 & 4 & 5 & 6 & 7 \\ \text { Very } & \text { Strongly } & \text { Somewhat } & \text { Neutral } & \text { Somewhat } & \text { Strongly } & \text { Very } \\ \text { Strongly } & \text { Disongly } \\ \text { Disagree } & \text { Disagree } & \text { Disagree } & & \text { Agree } & \text { Agree }\end{array}$

Ever since I was charged, I have a special person I can share my happiness and sadness with

$\begin{array}{ccccccc}1 & 2 & 3 & 4 & 5 & 6 & 7 \\ \text { Very } & \text { Strongly } & \text { Somewhat } & \text { Neutral } & \text { Somewhat } & \text { Strongly } & \text { Very } \\ \text { Strongly } & \text { Disagree } & \text { Disagree } & \text { Agree } & \text { Strongly } \\ \text { Disagree } & \text { Disaree }\end{array}$

Did you share your happiness and sadness with your special person before you were charged?

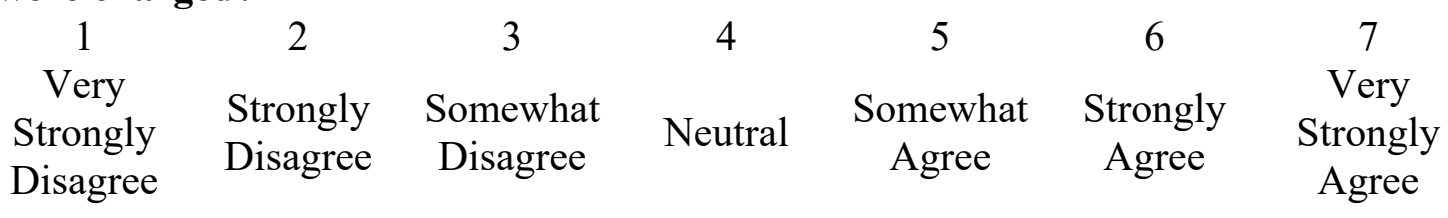

Ever since I was charged, my family really tries to help me

$\begin{array}{ccccccc}1 & 2 & 3 & 4 & 5 & 6 & 7 \\ \text { Very } & \text { Strongly } & \text { Somewhat } & \text { Neutral } & \text { Somewhat } & \text { Strongly } & \text { Very } \\ \text { Strongly } & \text { Disagree } & \text { Disagree } & & \text { Agree } & \text { Atrongly } \\ \text { Disagree } & \text { Disaree }\end{array}$

Did your family really try to help you before you were charged?

$\begin{array}{ccccccc}1 & 2 & 3 & 4 & 5 & 6 & 7 \\ \text { Very } & \text { Strongly } & \text { Somewhat } & \text { Neutral } & \text { Somewhat } & \text { Strongly } & \text { Very } \\ \text { Strongly } & \text { Disagree } & \text { Disagree } & \text { Agree } & \text { Strongly } \\ \text { Disagree } & \text { Disree }\end{array}$


Ever since I was charged, I get the emotional help and support I need from my family

$\begin{array}{ccccccc}1 & 2 & 3 & 4 & 5 & 6 & 7 \\ \text { Very } & \text { Strongly } & \text { Somewhat } & \text { Neutral } & \text { Somewhat } & \text { Strongly } & \text { Very } \\ \text { Strongly } & \text { Disagree } & \text { Disagree } & \text { Agree } & \text { Agree } \\ \text { Disagree } & \text { Disaree } & & & & & \text { Agree }\end{array}$

Did you get the emotional support you needed from your family before you were charged?

$\begin{array}{ccccccc}1 & 2 & 3 & 4 & 5 & 6 & 7 \\ \text { Very } & \text { Strongly } & \text { Somewhat } & \text { Neutral } & \text { Somewhat } & \text { Strongly } & \text { Very } \\ \text { Strongly } & \text { Disagree } & \text { Disagree } & \text { Agree } & \text { Strongly } \\ \text { Disagree } & \text { Disagee }\end{array}$

Ever since I was charged, I have a special person who comforts me

$\begin{array}{ccccccc}1 & 2 & 3 & 4 & 5 & 6 & 7 \\ \text { Very } & \text { Strongly } & \text { Somewhat } & \text { Neutral } & \text { Somewhat } & \text { Strongly } & \text { Very } \\ \text { Strongly } & \text { Disongly } \\ \text { Disagree } & \text { Disagree } & \text { Disagree } & & \text { Agree } & \begin{array}{c}\text { Agree } \\ \text { A }\end{array}\end{array}$

Did your special person comfort you before you were charged?

$\begin{array}{ccccccc}1 & 2 & 3 & 4 & 5 & 6 & 7 \\ \text { Very } & \text { Strongly } & \text { Somewhat } & \text { Neutral } & \text { Somewhat } & \text { Strongly } & \text { Very } \\ \text { Strongly } & \text { Disagree } & \text { Disagree } & \text { Agree } & \text { Atrongly } \\ \text { Disagree } & \text { Disaree }\end{array}$

Ever since I was charged, my friends really try to help me

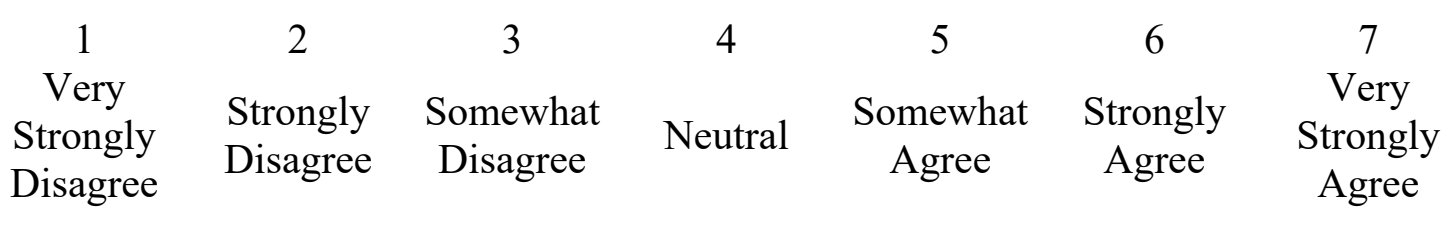

Did your friends really try to help you before you were charged?

\begin{tabular}{|c|c|c|c|c|c|c|}
\hline 1 & 2 & 3 & 4 & 5 & 6 & 7 \\
\hline $\begin{array}{c}\text { Very } \\
\text { Strongly } \\
\text { Disagree }\end{array}$ & $\begin{array}{l}\text { Strongly } \\
\text { Disagree }\end{array}$ & $\begin{array}{l}\text { Somewhat } \\
\text { Disagree }\end{array}$ & Neutral & $\begin{array}{c}\text { Somewhat } \\
\text { Agree }\end{array}$ & $\begin{array}{l}\text { Strongly } \\
\text { Agree }\end{array}$ & $\begin{array}{c}\text { Very } \\
\text { Strongly } \\
\text { Agree }\end{array}$ \\
\hline
\end{tabular}


Ever since I was charged, I can count on my friends when things are going badly

\begin{tabular}{|c|c|c|c|c|c|c|}
\hline 1 & 2 & 3 & 4 & 5 & 6 & 7 \\
\hline $\begin{array}{c}\text { Very } \\
\text { Strongly } \\
\text { Disagree }\end{array}$ & $\begin{array}{l}\text { Strongly } \\
\text { Disagree }\end{array}$ & $\begin{array}{l}\text { Somewhat } \\
\text { Disagree }\end{array}$ & Neutral & $\begin{array}{c}\text { Somewhat } \\
\text { Agree }\end{array}$ & $\begin{array}{l}\text { Strongly } \\
\text { Agree }\end{array}$ & $\begin{array}{c}\text { Very } \\
\text { Strongly } \\
\text { Agree }\end{array}$ \\
\hline
\end{tabular}

Could you could on your friends before you were charged?

\begin{tabular}{|c|c|c|c|c|c|c|}
\hline 1 & 2 & 3 & 4 & 5 & 6 & 7 \\
\hline $\begin{array}{c}\text { Very } \\
\text { Strongly } \\
\text { Disagree }\end{array}$ & $\begin{array}{l}\text { Strongly } \\
\text { Disagree }\end{array}$ & $\begin{array}{c}\text { Somewhat } \\
\text { Disagree }\end{array}$ & $\mathrm{Neu}$ & $\begin{array}{c}\text { Somewhat } \\
\text { Agree }\end{array}$ & $\begin{array}{c}\text { Strongly } \\
\text { Agree }\end{array}$ & $\begin{array}{c}\text { Very } \\
\text { Strongly } \\
\text { Agree }\end{array}$ \\
\hline
\end{tabular}

Ever since I was charged, I can talk to my family about my problems

\begin{tabular}{|c|c|c|c|c|c|c|}
\hline 1 & 2 & 3 & 4 & 5 & 6 & 7 \\
\hline $\begin{array}{c}\text { Very } \\
\text { Strongly } \\
\text { Disagree }\end{array}$ & $\begin{array}{l}\text { Strongly } \\
\text { Disagree }\end{array}$ & $\begin{array}{l}\text { Somewhat } \\
\text { Disagree }\end{array}$ & Neutral & $\begin{array}{c}\text { Somewhat } \\
\text { Agree }\end{array}$ & $\begin{array}{c}\text { Strongly } \\
\text { Agree }\end{array}$ & $\begin{array}{c}\text { Very } \\
\text { Strongly } \\
\text { Agree }\end{array}$ \\
\hline
\end{tabular}

Could you talk to your family about your problems before you were charged?

\begin{tabular}{|c|c|c|c|c|c|c|}
\hline 1 & 2 & 3 & 4 & 5 & 6 & 7 \\
\hline $\begin{array}{c}\text { Very } \\
\text { Strongly } \\
\text { Disagree }\end{array}$ & $\begin{array}{l}\text { Strongly } \\
\text { Disagree }\end{array}$ & $\begin{array}{l}\text { Somewhat } \\
\text { Disagree }\end{array}$ & Neutral & $\begin{array}{c}\text { Somewhat } \\
\text { Agree }\end{array}$ & $\begin{array}{c}\text { Strongly } \\
\text { Agree }\end{array}$ & $\begin{array}{c}\text { Very } \\
\text { Strongly } \\
\text { Agree }\end{array}$ \\
\hline
\end{tabular}

Ever since I was charged, I have friends I can share my happiness and sadness with

\begin{tabular}{|c|c|c|c|c|c|c|}
\hline 1 & 2 & 3 & 4 & 5 & 6 & 7 \\
\hline $\begin{array}{c}\text { Very } \\
\text { Strongly } \\
\text { Disagree }\end{array}$ & $\begin{array}{l}\text { Strongly } \\
\text { Disagree }\end{array}$ & $\begin{array}{l}\text { Somewhat } \\
\text { Disagree }\end{array}$ & Neutral & $\begin{array}{c}\text { Somewhat } \\
\text { Agree }\end{array}$ & $\begin{array}{c}\text { Strongly } \\
\text { Agree }\end{array}$ & $\begin{array}{c}\text { Very } \\
\text { Strongly } \\
\text { Agree }\end{array}$ \\
\hline
\end{tabular}

Did you have friends you could share your happiness and sadness with before you were charged?

\begin{tabular}{|c|c|c|c|c|c|c|}
\hline & & & & & & \\
\hline Ve & & & & & & \\
\hline $\begin{array}{l}\text { Strongly } \\
\text { Disagree }\end{array}$ & $\begin{array}{l}\text { Strongly } \\
\text { Disagree }\end{array}$ & $\begin{array}{l}\text { Somewhat } \\
\text { Disagree }\end{array}$ & Neutral & $\begin{array}{c}\text { Somewhat } \\
\text { Agree }\end{array}$ & $\begin{array}{c}\text { Strongly } \\
\text { Agree }\end{array}$ & $\begin{array}{c}\text { Strongly } \\
\text { Agree }\end{array}$ \\
\hline
\end{tabular}


Ever since I was charged, I have someone special in my life who cares about my feelings

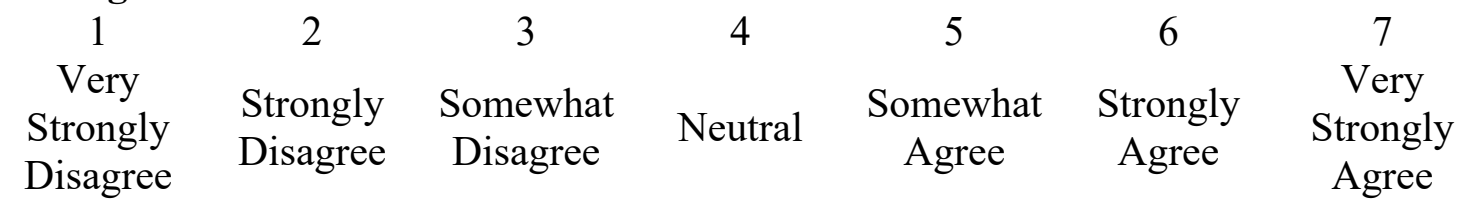

Did your special person care about your feelings before you were charged?

\begin{tabular}{|c|c|c|c|c|c|c|}
\hline 1 & 2 & 3 & 4 & 5 & 6 & 7 \\
\hline $\begin{array}{c}\text { Very } \\
\text { Strongly } \\
\text { Disagree }\end{array}$ & $\begin{array}{l}\text { Strongly } \\
\text { Disagree }\end{array}$ & $\begin{array}{c}\text { Somewhat } \\
\text { Disagree }\end{array}$ & Neutral & $\begin{array}{c}\text { Somewhat } \\
\text { Agree }\end{array}$ & $\begin{array}{c}\text { Strongly } \\
\text { Agree }\end{array}$ & $\begin{array}{c}\text { Very } \\
\text { Strongly } \\
\text { Agree }\end{array}$ \\
\hline
\end{tabular}

Ever since I was charged, my family is willing to help me make decisions

\begin{tabular}{|c|c|c|c|c|c|c|}
\hline 1 & 2 & 3 & 4 & 5 & 6 & 7 \\
\hline $\begin{array}{c}\text { Very } \\
\text { Strongly } \\
\text { Disagree }\end{array}$ & $\begin{array}{l}\text { Strongly } \\
\text { Disagree }\end{array}$ & $\begin{array}{l}\text { Somewhat } \\
\text { Disagree }\end{array}$ & Neutral & $\begin{array}{c}\text { Somewhat } \\
\text { Agree }\end{array}$ & $\begin{array}{c}\text { Strongly } \\
\text { Agree }\end{array}$ & $\begin{array}{c}\text { Very } \\
\text { Strongly } \\
\text { Agree }\end{array}$ \\
\hline
\end{tabular}

Was your family willing to help you make decisions before you were charged?

$\begin{array}{ccccccc}1 & 2 & 3 & 4 & 5 & 6 & 7 \\ \text { Very } & \text { Strongly } & \text { Somewhat } & \text { Neutral } & \text { Somewhat } & \text { Strongly } & \text { Very } \\ \text { Strongly } & \text { Disagree } & \text { Disagree } & \text { Agree } & \text { Agree } & \begin{array}{c}\text { Strongly } \\ \text { Agree }\end{array}\end{array}$

Ever since I was charged, I can talk about my problems with my friends

\begin{tabular}{|c|c|c|c|c|c|c|}
\hline 1 & 2 & 3 & 4 & 5 & 6 & 7 \\
\hline $\begin{array}{c}\text { Very } \\
\text { Strongly } \\
\text { Disagree }\end{array}$ & $\begin{array}{l}\text { Strongly } \\
\text { Disagree }\end{array}$ & $\begin{array}{l}\text { Somewhat } \\
\text { Disagree }\end{array}$ & Neutral & $\begin{array}{c}\text { Somewhat } \\
\text { Agree }\end{array}$ & $\begin{array}{l}\text { Strongly } \\
\text { Agree }\end{array}$ & $\begin{array}{c}\text { Very } \\
\text { Strongly } \\
\text { Agree }\end{array}$ \\
\hline
\end{tabular}

Could you talk to your friends about your problems before you were charged?

\begin{tabular}{|c|c|c|c|c|c|c|}
\hline 1 & 2 & 3 & 4 & 5 & 6 & 7 \\
\hline $\begin{array}{c}\text { Very } \\
\text { Strongly } \\
\text { Disagree }\end{array}$ & $\begin{array}{l}\text { Strongly } \\
\text { Disagree }\end{array}$ & $\begin{array}{c}\text { Somewhat } \\
\text { Disagree }\end{array}$ & Neutral & $\begin{array}{c}\text { Somewhat } \\
\text { Agree }\end{array}$ & $\begin{array}{l}\text { Strongly } \\
\text { Agree }\end{array}$ & $\begin{array}{c}\text { Very } \\
\text { Strongly } \\
\text { Agree }\end{array}$ \\
\hline
\end{tabular}




\section{Appendix I}

\section{Centre for Addiction and Mental Health Consent Forms}

\section{Questionnaire Information Form for Participants 16 or older}

Title of Study: Understanding the Experiences of Young Offenders and their Parents

\section{Study Investigators:}

Dr. Tracey Skilling

Clinician Scientist/Psychologist

Adolescent Service, CAMH

Child, Youth, and Family Program

tracey.skilling@camh.ca

416-535-8501 ext. 34578

\author{
Ms. Elisabeth Leroux \\ PhD Student \\ Carleton University \\ elisabeth.leroux@carleton.ca
}

Purpose of the Study: The purpose of the study is to understand the experiences of adolescents involved in the criminal justice system and their parents. This study is largely being conducted by a research student (Elisabeth Leroux) who will be using the information you provide for her $\mathrm{PhD}$ dissertation.

Procedure: Should you wish to participate, you will be asked to complete a self report questionnaire package that will take approximately 45 minutes to complete. We'll ask you some questions about your family, your friends, and your feelings.

Protection of Personal Information: At no time during this study will any of your personal information, including name, address, and birth date, be recorded. All of the answers you provide will be kept confidential and cannot be linked to any other information that may have been previously recorded while you have been at CAMH. Any details you share about yourself will remain strictly confidential, and will only be used for research purposes.

Whether you participate in this study or not will not influence any legal or treatment related decision. None of the information you provide will be recorded in your (or your child's) clinical file or shared with your (or your child's) clinicians.

Compensation: You will be compensated $\$ 20$ for your participation and you will have to sign confirming you received it.

Voluntary Participation: Your participation in this study is completely voluntary. That means you may refuse to participate and, if you do agree to participate, you may decide to stop at any time. You may also choose not to answer specific questions. Your decision to accept or refuse to participate in the study will in no way affect your experiences or record within the youth court system or your future access to services at CAMH (or anywhere else). 
Questions: If you have any questions or concerns about the study - please ask them. If you have any further questions that you don't believe have been answered or questions in the future, you may contact Elisabeth Leroux at elisabeth.leroux@carleton.ca or Dr. Skilling at the CAMH number above or tracey.skilling@camh.ca. Additionally, Dr. Padraig Darby, Chair, Research Ethics Board, Centre for Addiction and Mental Health, may be contacted by research participants to discuss their rights. Dr. Darby may be reached by telephone at 416-535-8501 ext. 36876 .

Do you agree to participate in this study? $\quad \square$ Yes $\quad \square$ No

Would you like a copy of this consent form? $\quad \square$ Yes $\quad \square$ No

Signature of Participant Date

Signature of Witness Date

As part of the Research Services Quality Assurance Program, this study may be monitored and/or audited by a member of the Quality Assurance Team. Your research records and CAMH records may be reviewed during which confidentiality will be maintained as per CAMH policies and extent permitted by law 


\section{Questionnaire Information Form for Parents of Participants under 16 years}

Title of Study: Understanding the Experiences of Young Offenders and their Parents

\section{Study Investigators:}

Dr. Tracey Skilling Clinician Scientist/Psychologist

Adolescent Service, CAMH

Child, Youth, and Family Program tracey.skilling@camh.ca

416-535-8501 ext. 34578

\author{
Ms. Elisabeth Leroux \\ PhD Student \\ Carleton University \\ elisabeth.leroux@carleton.ca
}

Purpose of the Study: The purpose of the study is to understand the experiences of adolescents involved in the criminal justice system and their parents. This study is largely being conducted by a research student (Elisabeth Leroux) who will be using the information you provide for her $\mathrm{PhD}$ dissertation.

Procedure: Should you wish to participate, you will be asked to complete a self report questionnaire package that will take approximately 45 minutes to complete. We'll ask you some questions about your family, your friends, and your feelings.

Protection of Personal Information: At no time during this study will any of your personal information, including name, address, and birth date, be recorded. All of the answers you provide will be kept confidential and cannot be linked to any other information that may have been previously recorded while you have been at CAMH. Any details you share about yourself will remain strictly confidential, and will only be used for research purposes.

Whether you participate in this study or not will not influence any legal or treatment related decision. None of the information you provide will be recorded in your (or your child's) clinical file or shared with your (or your child's) clinicians.

Compensation: You will be compensated $\$ 20$ for your participation and you will have to sign confirming you received it.

Voluntary Participation: Your participation in this study is completely voluntary. That means you may refuse to participate and, if you do agree to participate, you may decide to stop at any time. You may also choose not to answer specific questions. Your decision to accept or refuse to participate in the study will in no way affect your experiences or record within the youth court system or your future access to services at CAMH (or anywhere else). 
Questions: If you have any questions or concerns about the study - please ask them. If you have any further questions that you don't believe have been answered or questions in the future, you may contact Elisabeth Leroux at elisabeth.leroux@carleton.ca or Dr. Skilling at the CAMH number above or tracey.skilling@camh.ca. Additionally, Dr. Padraig Darby, Chair, Research Ethics Board, Centre for Addiction and Mental Health, may be contacted by research participants to discuss their rights. Dr. Darby may be reached by telephone at 416-535-8501 ext. 36876 .

Do you agree to allow your child to participate in this study? $\square$ Yes No

Would you like a copy of this consent form? $\quad \square$ Yes No

Name of person under 16 years:
Signature of Parent/Guardian
Date

Signature of Witness

Date

As part of the Research Services Quality Assurance Program, this study may be monitored and/or audited by a member of the Quality Assurance Team. Your research records and CAMH records may be reviewed during which confidentiality will be maintained as per CAMH policies and extent permitted by law 


\section{Questionnaire Information Form for Participants under 16 years}

Title of Study: Understanding the Experiences of Young Offenders and their Parents

\section{Study Investigators:}

Dr. Tracey Skilling

Clinician Scientist/Psychologist

Adolescent Service, CAMH

Child, Youth, and Family Program

tracey.skilling@camh.ca

416-535-8501 ext. 34578
Ms. Elisabeth Leroux

PhD Student

Carleton University

elisabeth.leroux@carleton.ca

Study: The purpose of the study is to understand the experiences of adolescents involved in the criminal justice system and their parents. This study is largely being conducted by a research student (Elisabeth Leroux) who will be using the information you provide for her $\mathrm{PhD}$ dissertation.

Procedure: Should you wish to participate, you will be asked to complete a self report questionnaire package that will take approximately 45 minutes to complete. We'll ask you some questions about your family, your friends, and your feelings.

Protection of Personal Information: At no time during this study will any of your personal information, including name, address, and birth date, be recorded. All of the answers you provide will be kept confidential and cannot be linked to any other information that may have been previously recorded while you have been at CAMH. Any details you share about yourself will remain strictly confidential, and will only be used for research purposes.

Whether you participate in this study or not will not influence any legal or treatment related decision. None of the information you provide will be recorded in your (or your child's) clinical file or shared with your (or your child's) clinicians.

Compensation: You will be compensated $\$ 20$ for your participation and you will have to sign confirming you received it.

Voluntary Participation: Your participation in this study is completely voluntary. That means you may refuse to participate and, if you do agree to participate, you may decide to stop at any time. You may also choose not to answer specific questions. Your decision to accept or refuse to participate in the study will in no way affect your experiences or record within the youth court system or your future access to services at CAMH (or anywhere else). 
Questions: If you have any questions or concerns about the study - please ask them. If you have any further questions that you don't believe have been answered or questions in the future, you may contact Elisabeth Leroux at elisabeth.leroux@carleton.ca or Dr. Skilling at the CAMH number above or tracey.skilling@camh.ca. Additionally, Dr. Padraig Darby, Chair, Research Ethics Board, Centre for Addiction and Mental Health, may be contacted by research participants to discuss their rights. Dr. Darby may be reached by telephone at 416-535-8501 ext. 36876.

Do you agree to participate in this study? $\quad \square \quad$ Yes $\square$ No

Do you have permission from your parent/guardian to participate? $\square$ Yes $\square$ No

Would you like a copy of this assent form?

Yes $\square$ No

Name of person under 16 years:

As part of the Research Services Quality Assurance Program, this study may be monitored and/or audited by a member of the Quality Assurance Team. Your research records and $C A M H$ records may be reviewed during which confidentiality will be maintained as per CAMH policies and extent permitted by law 


\section{$\underline{\text { Interview Information Form for Participants } 16 \text { years or older }}$}

Title of Study: Understanding the Experiences of Young Offenders and their Parents

\section{Study Investigators:}

Dr. Tracey Skilling Clinician Scientist/Psychologist

Adolescent Service, CAMH

Child, Youth, and Family Program

tracey.skilling@camh.ca

416-535-8501 ext. 34578

$\begin{array}{ll}\text { Ms. Elisabeth Leroux } & \text { Dr. Michael Seto } \\ \text { PhD Student } & \text { University of Ottawa } \\ \text { Carleton University } & \text { Institute of Mental Health } \\ \text { elisabeth.leroux@carleton.ca } & \text { Research } \\ & \begin{array}{l}\text { michael.seto@theroyal.ca } \\ 613-345-1461 \text { ext. 2605 }\end{array}\end{array}$

613-345-1461 ext. 2605

Purpose of the Study: The purpose of the study is to understand the experiences of adolescents involved in the criminal justice system and their parents. This study is largely being conducted by a research student (Elisabeth Leroux) who will be using the information you provide for her $\mathrm{PhD}$ dissertation.

Procedure: Should you wish to participate, you will be asked to answer some interview questions. The interview will probably range from 45 minutes up to $11 / 2$ hours, but it depends on how much you decide to talk. You will be asked questions about your life before you were charged with your offence, and how your life has changed since you have been charged.

Protection of Personal Information: At no time during this study will any of your personal information, including name, address, and birth date, be recorded. All of the answers you provide will be kept confidential and cannot be linked to any other information that may have been previously recorded while you have been at CAMH. Voice recordings will be transcribed and then deleted. The electronic transcriptions will be anonymized and kept on a secure device. Any details you share about yourself will remain strictly confidential, and will only be used for research purposes.

Whether you participate in this study or not will not influence any legal or treatment related decision. None of the information you provide will be recorded in your (or your child's) clinical file or shared with your (or your child's) clinicians.

Although we want to keep all of the information you share with us private, if you directly tell us about any incidents of abuse (sexual, physical, emotional) or neglect about yourself (or your child) or any other minor we are legally obligated to report it. If you answer the anonymous questions about abuse or neglect this will not be reported.

Compensation: You will be compensated $\$ 30$ for your participation and you will have to sign confirming you received it. 
Voluntary Participation: Your participation in this study is completely voluntary. That means you may refuse to participate and, if you do agree to participate, you may decide to stop at any time. You may also choose not to answer specific questions. Your decision to accept or refuse to participate in the study will in no way affect your experiences or record within the youth court system or your future access to services at CAMH (or anywhere else).

Questions: If you have any questions or concerns about the study - please ask them. If you have any further questions that you don't believe have been answered or questions in the future, you may contact Elisabeth Leroux at elisabeth.leroux@carleton.ca or Dr. Skilling at the CAMH number above or tracey.skilling@camh.ca. Additionally, Dr. Robert Levitan, Chair, Research Ethics Board, Centre for Addiction and Mental Health, may be contacted by research participants to discuss their rights. Dr. Levitan may be reached by telephone at 416-535-8501 ext. 34020.

Do you agree to participate in this study? $\quad \square$ Yes $\quad \square$ No

Would you like a copy of this consent form? $\quad \square$ Yes $\quad \square$ No

Signature of Participant Date

Signature of Witness

Date

As part of the Research Services Quality Assurance Program, this study may be monitored and/or audited by a member of the Quality Assurance Team. Your research records and $C A M H$ records may be reviewed during which confidentiality will be maintained as per CAMH policies and extent permitted by law. 


\section{Interview Information Form for Parents of Participants under 16 years}

Title of Study: Understanding the Experiences of Young Offenders and their Parents

\section{Study Investigators:}

\author{
Dr. Tracey Skilling \\ Clinician Scientist/Psychologist \\ Adolescent Service, CAMH \\ Child, Youth, and Family Program \\ tracey.skilling@camh.ca \\ 416-535-8501 ext. 34578
}

\author{
Ms. Elisabeth Leroux \\ PhD Student \\ Carleton University \\ elisabeth.leroux@carleton.ca
}

Purpose of the Study: The purpose of the study is to understand the experiences of adolescents involved in the criminal justice system and their parents. This study is largely being conducted by a research student (Elisabeth Leroux) who will be using the information you provide for her $\mathrm{PhD}$ dissertation.

Procedure: Should you wish to participate, you will be asked to answer some interview questions. The interview will probably range from 45 minutes up to $11 / 2$ hours, but it depends on how much you decide to talk. You will be asked questions about your life before you were charged with your offence, and how your life has changed since you have been charged.

Protection of Personal Information: At no time during this study will any of your personal information, including name, address, and birth date, be recorded. All of the answers you provide will be kept confidential and cannot be linked to any other information that may have been previously recorded while you have been at CAMH. Voice recordings will be transcribed and then deleted. The electronic transcriptions will be anonymized and kept on a secure device. Any details you share about yourself will remain strictly confidential, and will only be used for research purposes.

Whether you participate in this study or not will not influence any legal or treatment related decision. None of the information you provide will be recorded in your (or your child's) clinical file or shared with your (or your child's) clinicians.

Although we want to keep all of the information you share with us private, if you directly tell us about any incidents of abuse (sexual, physical, emotional) or neglect about yourself (or your child) or any other minor we are legally obligated to report it. If you answer the anonymous questions about abuse or neglect this will not be reported.

Compensation: You will be compensated $\$ 30$ for your participation and you will have to sign confirming you received it. 
Voluntary Participation: Your participation in this study is completely voluntary. That means you may refuse to participate and, if you do agree to participate, you may decide to stop at any time. You may also choose not to answer specific questions. Your decision to accept or refuse to participate in the study will in no way affect your experiences or record within the youth court system or your future access to services at CAMH (or anywhere else).

Questions: If you have any questions or concerns about the study - please ask them. If you have any further questions that you don't believe have been answered or questions in the future, you may contact Elisabeth Leroux at elisabeth.leroux@carleton.ca or Dr. Skilling at the CAMH number above or tracey.skilling@camh.ca. Additionally, Dr. Robert Levitan, Chair, Research Ethics Board, Centre for Addiction and Mental Health, may be contacted by research participants to discuss their rights. Dr. Levitan may be reached by telephone at 416-535-8501 ext. 34020.

Do you agree to allow your child to participate in this study? $\quad \square$ Yes $\quad \square$ No Would you like a copy of this consent form? $\quad \square$ Yes $\quad \square$ No Name of person under 16 years:

As part of the Research Services Quality Assurance Program, this study may be monitored and/or audited by a member of the Quality Assurance Team. Your research records and $C A M H$ records may be reviewed during which confidentiality will be maintained as per CAMH policies and extent permitted by law. 


\section{$\underline{\text { Interview Information Form for Participants under } 16 \text { years }}$}

Title of Study: Understanding the Experiences of Young Offenders and their Parents

\section{Study Investigators:}

Dr. Tracey Skilling

Clinician Scientist/Psychologist

Adolescent Service, CAMH

Child, Youth, and Family Program

tracey.skilling@camh.ca

416-535-8501 ext. 34578

\author{
Ms. Elisabeth Leroux \\ PhD Student \\ Dr. Michael Seto \\ University of Ottawa \\ Institute of Mental Health \\ Carleton University \\ elisabeth.leroux@carleton.ca \\ Research \\ michael.seto@theroyal.ca \\ 613-345-1461 ext. 2605
}

Purpose of the Study: The purpose of the study is to understand the experiences of adolescents involved in the criminal justice system and their parents. This study is largely being conducted by a research student (Elisabeth Leroux) who will be using the information you provide for her $\mathrm{PhD}$ dissertation.

Procedure: Should you wish to participate, you will be asked to answer some interview questions. The interview will probably range from 45 minutes up to $11 / 2$ hours, but it depends on how much you decide to talk. You will be asked questions about your life before you were charged with your offence, and how your life has changed since you have been charged.

Protection of Personal Information: At no time during this study will any of your personal information, including name, address, and birth date, be recorded. All of the answers you provide will be kept confidential and cannot be linked to any other information that may have been previously recorded while you have been at CAMH. Voice recordings will be transcribed and then deleted. The electronic transcriptions will be anonymized and kept on a secure device. Any details you share about yourself will remain strictly confidential, and will only be used for research purposes.

Whether you participate in this study or not will not influence any legal or treatment related decision. None of the information you provide will be recorded in your (or your child's) clinical file or shared with your (or your child's) clinicians.

Although we want to keep all of the information you share with us private, if you directly tell us about any incidents of abuse (sexual, physical, emotional) or neglect about yourself (or your child) or any other minor we are legally obligated to report it. If you answer the anonymous questions about abuse or neglect this will not be reported.

Compensation: You will be compensated $\$ 30$ for your participation and you will have to sign confirming you received it. 
Voluntary Participation: Your participation in this study is completely voluntary. That means you may refuse to participate and, if you do agree to participate, you may decide to stop at any time. You may also choose not to answer specific questions. Your decision to accept or refuse to participate in the study will in no way affect your experiences or record within the youth court system or your future access to services at CAMH (or anywhere else).

Questions: If you have any questions or concerns about the study - please ask them. If you have any further questions that you don't believe have been answered or questions in the future, you may contact Elisabeth Leroux at elisabeth.leroux@carleton.ca or Dr. Skilling at the CAMH number above or tracey.skilling@camh.ca. Additionally, Dr. Robert Levitan, Chair, Research Ethics Board, Centre for Addiction and Mental Health, may be contacted by research participants to discuss their rights. Dr. Levitan may be reached by telephone at 416-535-8501 ext. 34020.

Do you agree to participate in this study? $\quad \square$ Yes $\quad \square$ No

Do you have permission from your guardian to participate? $\square$ Yes $\square$ No

Would you like a copy of this assent form? $\quad \square$ Yes $\quad \square$ No

Name of person under 16 years:

As part of the Research Services Quality Assurance Program, this study may be monitored and/or audited by a member of the Quality Assurance Team. Your research records and $C A M H$ records may be reviewed during which confidentiality will be maintained as per CAMH policies and extent permitted by law. 


\section{Appendix J}

\section{The Royal Ottawa Consent Forms}

\section{Questionnaire Information Form for Participants 16 or older}

Title of Study: Understanding the Experiences of Young Offenders and their Parents

\section{Study Investigators:}

\author{
Ms. Elisabeth Leroux \\ PhD Student \\ Carleton University \\ elisabeth.leroux@carleton.ca
}

\author{
Dr. Michael Seto \\ University of Ottawa Institute of \\ Mental Health Research \\ michael.seto@theroyal.ca \\ 613-345-1461 ext. 2605
}

\author{
Dr. Greg Motayne \\ Family Court Clinic, IFP \\ Royal Ottawa Mental Health \\ Centre \\ greg.motayne@theroyal.ca \\ 613-722-6521 ext. 6400
}

Purpose of the Study: The purpose of the study is to understand the experiences of adolescents involved in the criminal justice system and their parents. We are also interested in examining differences between adolescents charged with a sexual offence and adolescents charged with non-sexual offences.

Procedure: You will complete a questionnaire using a laptop that will take approximately 45 minutes to complete. The questionnaire does include some questions about your sexual experiences, but it mostly focuses on your family, your friends, and your feelings.

Protection of Personal Information: At no time during this study will any of your personal information, including name, address, and birth date, be recorded. All of the answers you provide will be kept confidential and cannot be linked to any other information that may have been previously recorded while you have been at the Royal. Any details you share about yourself will remain strictly confidential, and will only be used for research purposes.

Whether you participate in this study or not will not influence any legal or treatment related decision. None of the information you provide will be recorded in your (or your child's) clinical file or shared with your (or your child's) clinicians.

Although we want to keep all of the information you share with us private, there are certain things that we are legally obligated to report. These include:

- Any incidents of abuse (sexual, physical, emotional) or neglect about yourself

- Any incidents of abuse (sexual, physical, emotional) or neglect about someone under 18

- Any imminent risk of harm to yourself

- Any imminent risk of harm to someone else

- Any sexual misconduct by a regulated 
If you disclose any of this information, we will report it to Dr. Motayne, the Clinic Psychologist.

You CAN talk about incidents of abuse or neglect that have already been reported. And, you CAN answer the anonymous questions about abuse or neglect and it will not be reported.

Compensation: You will be compensated $\$ 20$ for your participation and you will have to sign confirming you received it.

Voluntary Participation: Your participation in this study is completely voluntary. That means you may refuse to participate and, if you do agree to participate, you may decide to stop at any time. You may also choose not to answer specific questions. Your decision to accept or refuse to participate in the study will in no way affect your experiences or record within the youth court system or your future access to services at the Royal (or anywhere else).

Questions: If you have any questions or concerns about the study - please ask them. If you have any further questions that you don't believe have been answered or questions in the future, you may contact Elisabeth Leroux at elisabeth.leroux@carleton.ca or Dr. Michael Seto at the Royal number above. Additionally, the Chair of the Research Ethics Board, Royal Ottawa Health Care Group may be contacted by research participants to discuss their rights at (613) 722-6521 ext. 6214.

Do you agree to participate in this study?

$\square$ Yes

$\square$ No

Would you like a copy of this consent form?

$\square$ Yes

$\square$ No 


\section{Questionnaire Information Form for Parents of Participants under 16 years}

Title of Study: Understanding the Experiences of Young Offenders and their Parents

\section{Study Investigators:}

\author{
Ms. Elisabeth Leroux \\ PhD Student \\ Carleton University \\ elisabeth.leroux@carleton.ca
}

\author{
Dr. Michael Seto \\ University of Ottawa Institute of \\ Mental Health Research \\ michael.seto@theroyal.ca \\ 613-345-1461 ext.2605
}

\author{
Dr. Greg Motayne \\ Family Court Clinic, IFP \\ Royal Ottawa Mental Health \\ Centre \\ greg.motayne@theroyal.ca \\ 613-722-6521 ext. 6400
}

Purpose of the Study: The purpose of the study is to understand the experiences of adolescents involved in the criminal justice system and their parents. We are also interested in examining differences between adolescents charged with a sexual offence and adolescents charged with non-sexual offences.

Procedure: You will complete a questionnaire using a laptop that will take approximately 45 minutes to complete. The questionnaire does include some questions about your sexual experiences, but it mostly focuses on your family, your friends, and your feelings.

Protection of Personal Information: At no time during this study will any of your personal information, including name, address, and birth date, be recorded. All of the answers you provide will be kept confidential and cannot be linked to any other information that may have been previously recorded while you have been at the Royal. Any details you share about yourself will remain strictly confidential, and will only be used for research purposes.

Whether you participate in this study or not will not influence any legal or treatment related decision. None of the information you provide will be recorded in your (or your child's) clinical file or shared with your (or your child's) clinicians.

Although we want to keep all of the information you share with us private, there are certain things that we are legally obligated to report. These include:

- Any incidents of abuse (sexual, physical, emotional) or neglect about yourself

- Any incidents of abuse (sexual, physical, emotional) or neglect about someone under 18

- Any imminent risk of harm to yourself

- Any imminent risk of harm to someone else

- Any sexual misconduct by a regulated

If you disclose any of this information, we will report it to Dr. Motayne, the Clinic Psychologist. 
You CAN talk about incidents of abuse or neglect that have already been reported. And, you CAN answer the anonymous questions about abuse or neglect and it will not be reported.

Compensation: You will be compensated \$20 for your participation and you will have to sign confirming you received it.

Voluntary Participation: Your participation in this study is completely voluntary. That means you may refuse to participate and, if you do agree to participate, you may decide to stop at any time. You may also choose not to answer specific questions. Your decision to accept or refuse to participate in the study will in no way affect your experiences or record within the youth court system or your future access to services at the Royal (or anywhere else).

Questions: If you have any questions or concerns about the study - please ask them. If you have any further questions that you don't believe have been answered or questions in the future, you may contact Elisabeth Leroux at elisabeth.leroux@carleton.ca or Dr. Michael Seto at the Royal number above. Additionally, the Chair of the Research Ethics Board, Royal Ottawa Health Care Group may be contacted by research participants to discuss their rights at (613) 722-6521 ext. 6214.

Do you agree to participate in this study?

$\square$ Yes

$\square$ No

Would you like a copy of this consent form?

$\square$ Yes

$\square$ No

Name of person under 16 years:

Signature of Parent/Guardian

Date

Signature of Witness

Date 


\section{Questionnaire Information Form for Participants under 16 years}

Title of Study: Understanding the Experiences of Young Offenders and their Parents

\section{Study Investigators:}

\author{
Ms. Elisabeth Leroux \\ PhD Student \\ Carleton University \\ elisabeth.leroux@carleton.ca
}

\author{
Dr. Michael Seto \\ University of Ottawa Institute of \\ Mental Health Research \\ michael.seto@theroyal.ca \\ 613-345-1461 ext. 2605
}

\author{
Dr. Greg Motayne \\ Family Court Clinic, IFP \\ Royal Ottawa Mental Health \\ Centre \\ greg.motayne@theroyal.ca \\ 613-722-6521 ext. 6400
}

Purpose of the Study: The purpose of the study is to understand the experiences of adolescents involved in the criminal justice system and their parents. We are also interested in examining differences between adolescents charged with a sexual offence and adolescents charged with non-sexual offences.

Procedure: You will complete a questionnaire using a laptop that will take approximately 45 minutes to complete. The questionnaire does include some questions about your sexual experiences, but it mostly focuses on your family, your friends, and your feelings.

Protection of Personal Information: At no time during this study will any of your personal information, including name, address, and birth date, be recorded. All of the answers you provide will be kept confidential and cannot be linked to any other information that may have been previously recorded while you have been at the Royal. Any details you share about yourself will remain strictly confidential, and will only be used for research purposes.

Whether you participate in this study or not will not influence any legal or treatment related decision. None of the information you provide will be recorded in your (or your child's) clinical file or shared with your (or your child's) clinicians.

Although we want to keep all of the information you share with us private, there are certain things that we are legally obligated to report. These include:

- Any incidents of abuse (sexual, physical, emotional) or neglect about yourself

- Any incidents of abuse (sexual, physical, emotional) or neglect about someone under 18

- Any imminent risk of harm to yourself

- Any imminent risk of harm to someone else

- Any sexual misconduct by a regulated

If you disclose any of this information, we will report it to Dr. Motayne, the Clinic Psychologist. 
You CAN talk about incidents of abuse or neglect that have already been reported. And, you CAN answer the anonymous questions about abuse or neglect and it will not be reported.

Compensation: You will be compensated $\$ 20$ for your participation and you will have to sign confirming you received it.

Voluntary Participation: Your participation in this study is completely voluntary. That means you may refuse to participate and, if you do agree to participate, you may decide to stop at any time. You may also choose not to answer specific questions. Your decision to accept or refuse to participate in the study will in no way affect your experiences or record within the youth court system or your future access to services at the Royal (or anywhere else).

Questions: If you have any questions or concerns about the study - please ask them. If you have any further questions that you don't believe have been answered or questions in the future, you may contact Elisabeth Leroux at elisabeth.leroux@carleton.ca or Dr. Michael Seto at the Royal number above. Additionally, the Chair of the Research Ethics Board, Royal Ottawa Health Care Group may be contacted by research participants to discuss their rights at (613) 722-6521 ext. 6214.

Do you agree to participate in this study?

$\square$ Yes

$\square$ No

Would you like a copy of this consent form?

$\square$ Yes

$\square$ No

Name of person under 16 years: 


\section{$\underline{\text { Interview Information Form for Participants } 16 \text { years or older }}$}

Title of Study: Understanding the Experiences of Young Offenders and their Parents

\section{Study Investigators:}

\author{
Ms. Elisabeth Leroux \\ PhD Student \\ Carleton University \\ elisabeth.leroux@carleton.ca
}

\author{
Dr. Michael Seto \\ University of Ottawa Institute of \\ Mental Health Research \\ michael.seto@theroyal.ca \\ 613-345-1461 ext. 2605
}

\author{
Dr. Greg Motayne \\ Family Court Clinic, IFP \\ Royal Ottawa Mental Health \\ Centre \\ greg.motayne@theroyal.ca \\ 613-722-6521 ext. 6400
}

Purpose of the Study: The purpose of the study is to understand the experiences of adolescents involved in the criminal justice system and their parents.

Procedure: You will be asked a series of interview questions. The interview will be voice recorded and will range from 30 minutes up to 1 hour. You will be asked questions about the ways in which your life has changed since you (or your child) have been charged with a criminal offense.

Protection of Personal Information: At no time during this study will any of your personal information, including name, address, and birth date, be recorded. All of the answers you provide will be kept confidential and cannot be linked to any other information that may have been previously recorded while you have been at the Royal. Voice recordings will be transcribed and then deleted. The electronic transcriptions will be anonymized and kept on a secure device. Any details you share about yourself will remain strictly confidential, and will only be used for research purposes.

Whether you participate in this study or not will not influence any legal or treatment related decision. None of the information you provide will be recorded in your (or your child's) clinical file or shared with your (or your child's) clinicians.

Although we want to keep all of the information you share with us private, there are certain things that we are legally obligated to report. These include:

- Any incidents of abuse (sexual, physical, emotional) or neglect about yourself

- Any incidents of abuse (sexual, physical, emotional) or neglect about someone under 18

- Any imminent risk of harm to yourself

- Any imminent risk of harm to someone else

- Any sexual misconduct by a regulated

If you disclose any of this information, we will report it to Dr. Motayne, the Clinic Psychologist. 
You CAN talk about incidents of abuse or neglect that have already been reported. And, you CAN answer the anonymous questions about abuse or neglect and it will not be reported.

Compensation: You will be compensated $\$ 20$ for your participation and you will have to sign confirming you received it.

Voluntary Participation: Your participation in this study is completely voluntary. That means you may refuse to participate and, if you do agree to participate, you may decide to stop at any time. You may also choose not to answer specific questions. Your decision to accept or refuse to participate in the study will in no way affect your experiences or record within the youth court system or your future access to services at the Royal (or anywhere else).

Questions: If you have any questions or concerns about the study - please ask them. If you have any further questions that you don't believe have been answered or questions in the future, you may contact Elisabeth Leroux at elisabeth.leroux@carleton.ca or Dr. Michael Seto. Additionally, the Chair of the Research Ethics Board, Royal Ottawa Health Care Group may be contacted by research participants to discuss their rights at (613) 722 6521 ext. 6214.

Do you agree to participate in this study? $\quad \square$ Yes $\quad \square$ No

Would you like a copy of this consent form? $\quad \square$ Yes $\quad \square$ No

Signature of Participant Date

Signature of Witness Date 


\section{$\underline{\text { Interview Information Form for Parents of Participants under } 16 \text { years }}$}

Title of Study: Understanding the Experiences of Young Offenders and their Parents

\section{Study Investigators:}

\author{
Ms. Elisabeth Leroux \\ $\mathrm{PhD}$ Student \\ Carleton University \\ elisabeth.leroux@carleton.ca
}

Dr. Michael Seto
University of Ottawa Institute of
Mental Health Research
michael.seto@theroyal.ca
613-345-1461 ext. 2605

\author{
Dr. Greg Motayne \\ Family Court Clinic, IFP \\ Royal Ottawa Mental Health \\ Centre \\ greg.motayne@theroyal.ca \\ 613-722-6521 ext. 6400
}

Purpose of the Study: The purpose of the study is to understand the experiences of adolescents involved in the criminal justice system and their parents.

Procedure: You will be asked a series of interview questions. The interview will be voice recorded and will range from 30 minutes up to 1 hour. You will be asked questions about the ways in which your life has changed since you (or your child) have been charged with a criminal offense.

Protection of Personal Information: At no time during this study will any of your personal information, including name, address, and birth date, be recorded. All of the answers you provide will be kept confidential and cannot be linked to any other information that may have been previously recorded while you have been at the Royal. Voice recordings will be transcribed and then deleted. The electronic transcriptions will be anonymized and kept on a secure device. Any details you share about yourself will remain strictly confidential, and will only be used for research purposes.

Whether you participate in this study or not will not influence any legal or treatment related decision. None of the information you provide will be recorded in your (or your child's) clinical file or shared with your (or your child's) clinicians.

Although we want to keep all of the information you share with us private, there are certain things that we are legally obligated to report. These include:

- Any incidents of abuse (sexual, physical, emotional) or neglect about yourself

- Any incidents of abuse (sexual, physical, emotional) or neglect about someone under 18

- Any imminent risk of harm to yourself

- Any imminent risk of harm to someone else

- Any sexual misconduct by a regulated

If you disclose any of this information, we will report it to Dr. Motayne, the Clinic Psychologist. 
You CAN talk about incidents of abuse or neglect that have already been reported. And, you CAN answer the anonymous questions about abuse or neglect and it will not be reported.

Compensation: You will be compensated $\$ 20$ for your participation and you will have to sign confirming you received it.

Voluntary Participation: Your participation in this study is completely voluntary. That means you may refuse to participate and, if you do agree to participate, you may decide to stop at any time. You may also choose not to answer specific questions. Your decision to accept or refuse to participate in the study will in no way affect your experiences or record within the youth court system or your future access to services at the Royal (or anywhere else).

Questions: If you have any questions or concerns about the study - please ask them. If you have any further questions that you don't believe have been answered or questions in the future, you may contact Elisabeth Leroux at elisabeth.leroux@carleton.ca or Dr. Michael Seto. Additionally, the Chair of the Research Ethics Board, Royal Ottawa Health Care Group may be contacted by research participants to discuss their rights at (613) 722 6521 ext. 6214.

Do you agree to participate in this study? $\quad \square$ Yes $\quad \square$ No

Would you like a copy of this consent form? $\quad \square$ Yes $\quad \square$ No

Name of person under 16 years:
Signature of Parent/Guardian
Date 


\section{$\underline{\text { Interview Information Form for Participants under } 16 \text { years }}$}

Title of Study: Understanding the Experiences of Young Offenders and their Parents

\section{Study Investigators:}

\author{
Ms. Elisabeth Leroux \\ $\mathrm{PhD}$ Student \\ Carleton University \\ elisabeth.leroux@carleton.ca
}

Dr. Michael Seto
University of Ottawa Institute of
Mental Health Research
michael.seto@theroyal.ca
613-345-1461 ext. 2605

\author{
Dr. Greg Motayne \\ Family Court Clinic, IFP \\ Royal Ottawa Mental Health \\ Centre \\ greg.motayne@theroyal.ca \\ 613-722-6521 ext. 6400
}

Purpose of the Study: The purpose of the study is to understand the experiences of adolescents involved in the criminal justice system and their parents.

Procedure: You will be asked a series of interview questions. The interview will be voice recorded and will range from 30 minutes up to 1 hour. You will be asked questions about the ways in which your life has changed since you (or your child) have been charged with a criminal offense.

Protection of Personal Information: At no time during this study will any of your personal information, including name, address, and birth date, be recorded. All of the answers you provide will be kept confidential and cannot be linked to any other information that may have been previously recorded while you have been at the Royal. Voice recordings will be transcribed and then deleted. The electronic transcriptions will be anonymized and kept on a secure device. Any details you share about yourself will remain strictly confidential, and will only be used for research purposes.

Whether you participate in this study or not will not influence any legal or treatment related decision. None of the information you provide will be recorded in your (or your child's) clinical file or shared with your (or your child's) clinicians.

Although we want to keep all of the information you share with us private, there are certain things that we are legally obligated to report. These include:

- Any incidents of abuse (sexual, physical, emotional) or neglect about yourself

- Any incidents of abuse (sexual, physical, emotional) or neglect about someone under 18

- Any imminent risk of harm to yourself

- Any imminent risk of harm to someone else

- Any sexual misconduct by a regulated

If you disclose any of this information, we will report it to Dr. Motayne, the Clinic Psychologist. 
You CAN talk about incidents of abuse or neglect that have already been reported. And, you CAN answer the anonymous questions about abuse or neglect and it will not be reported.

Compensation: You will be compensated $\$ 20$ for your participation and you will have to sign confirming you received it.

Voluntary Participation: Your participation in this study is completely voluntary. That means you may refuse to participate and, if you do agree to participate, you may decide to stop at any time. You may also choose not to answer specific questions. Your decision to accept or refuse to participate in the study will in no way affect your experiences or record within the youth court system or your future access to services at the Royal (or anywhere else).

Questions: If you have any questions or concerns about the study - please ask them. If you have any further questions that you don't believe have been answered or questions in the future, you may contact Elisabeth Leroux at elisabeth.leroux@carleton.ca or Dr. Michael Seto. Additionally, the Chair of the Research Ethics Board, Royal Ottawa Health Care Group may be contacted by research participants to discuss their rights at (613) 722 6521 ext. 6214.

Do you agree to participate in this study? $\quad \square$ Yes $\quad \square$ No

Would you like a copy of this consent form? $\quad \square$ Yes $\quad \square$ No

Name of person under 16 years:

Signature of Participant Date




\section{Appendix K}

\section{Confirmation of Compensation}

Study Title: Understanding the Experiences of Young Offenders and their Parents

Investigators: Elisabeth Leroux (Ph.D. Student), Dr. Kelly Babchishin,

Dr. Greg Motayne, \& Dr. Michael Seto

CAMH REB\#: 118-2015

Royal REB\#: 2016-002

Carleton REB\#: 16-053

By initialing below, I confirm that I have been given:

\$20.00 for the survey I completed

$\$ 30.00$ for the interview I completed

Researcher initial:

Participant initial: 


\section{Appendix L}

\section{Centre for Addiction and Mental Health Participant Debrief Form \\ Resources for Research Participants}

\section{Have questions about your rights as a research participant?}

\section{Office of Research Ethics at CAMH}

If you have questions regarding your rights as research participants: Contact Dr.

Padraig Darby, Research Ethics Board Chair, CAMH

Telephone: 416-535-8501 ext. 36876

Email: padraig.darby@camh.ca

Need someone to talk to?

Ontario Mental Health Helpline (24-hours)

Personnel will listen, offer support and provide strategies to help meet your goals. They will also give you information about mental health services in Ontario.

Telephone: 1-866-531-2600 (free calling from anywhere within Canada)

\section{Kids Help Phone (24-hours)}

Professional counselors provide anonymous, confidential and non-judgemental support for children and youth 5-20 years old

Telephone: 1-800-668-6868 (free calling from anywhere within Canada)

Want to access mental health services in Toronto?

The Centre for Addition and Mental Health (CAMH) (1001 Queen Street West)

Provides assessment and intervention services for youth and adults

- CAMH uses a centralized intake process. To inquire about the availability of specific programs please call: 416-535-8501 (and then press 2).

The Hincks-Dellcrest Centre (440 Jarvis Street)

Provides outpatient services for youth up to the age of 17

- Contact the central intake line at: 416-924-1164 to begin the intake process

The Hospital for Sick Children (SickKids) (555 University Avenue)

Provides assessment and intervention services for children and adolescents

Teen Clinic

Phone: 416-813-5804

Contact person: Susan

Duncan, Patient Care

Information

Coordinator

\section{Substance Abuse Outreach Program}

Phone: (416) 813-5097

Contact person: Elnara

Zeinalov, Intake Coordinator
Substance Abuse Day

Treatment Program

Phone: (416) 813-7941, (416)

813-8854

Contact person: Paul Pereira,

Counsellor

Youthdale Psychiatric Crisis Services - Mobile response team.

- Contact: 416-363-9990 (24 hours a day, seven days a week) 


\section{Appendix M}

\section{The Royal Ottawa Participant Debrief Form}

\section{Resources for Research Participants}

\section{Have questions about your rights as a research participant?}

\section{Office of Research Ethics at the Royal}

If you have questions regarding your rights as research participants: Contact Dr. Pierre Blier, Research Ethics Chair, Royal Ottawa Health Care Group, Ottawa, ON.

Telephone: 613-722-6521 ext. 6944

Email: pierre.blier@theroyal.ca

\section{Need someone to talk to?}

Ontario Mental Health Helpline (24-hours)

Personnel will listen, offer support and provide strategies to help meet your goals. They will also give you information about mental health services in Ontario.

Telephone: 1-866-531-2600 (free calling from anywhere within Canada)

\section{Kids Help Phone (24-hours)}

Professional counselors provide anonymous, confidential and non-judgemental support for children and youth 5-20 years old

Telephone: 1-800-668-6868 (free calling from anywhere within Canada)

Want to access mental health services in Ottawa?

The Royal (1145 Carling Ave.)

Provides assessment and intervention services for youth and adults

- To inquire about the availability of specific programs at the Royal please call: 613-722-6521 or 1-800-987-6424

Youth Services Bureau of Ottawa (2301 Carling Ave.)

Provides community based services for youth

\section{Mental Health Services Hub}

Phone: (613) 562-3004

Contact person: Maureen Davidson, Intake

Services Coordinator

\author{
Youth and Family Counselling \\ Phone: (613) 562-3004 \\ Contact person: Mandy Leafloor, \\ Coordinator
}

\section{Youth Services Bureau of Ottawa - Crisis Line}

- Contact: (613) 260-2360 (24 hours a day, seven days a week)

The Children's Hospital of Eastern Ontario (CHEO) (401 Smyth Road) Provides assessment and intervention services for children and adolescents

- To inquire about the availability of specific mental health programs at CHEO please call: 613-737-7600 ext. 24966 


\section{Appendix N}

\section{Internalized Stigma Scale of Offending}

I feel out of place in the world because I'm an offender

$\begin{array}{cccc}1 & 2 & 3 & 4 \\ \text { Strongly Disagree } & \text { Disagree } & \text { Agree } & \text { Strongly Agree }\end{array}$

Being an offender has ruined my life
1
2
3
4
Strongly Disagree
Disagree
Agree
Strongly Agree

People who are not offenders themselves cannot understand me
1
2
Disagree
3
4
Strongly Disagree
Agree
Strongly Agree

I am embarrassed or ashamed because I am an offender

$\begin{array}{cccc}1 & 2 & 3 & 4 \\ \text { Strongly Disagree } & \text { Disagree } & \text { Agree } & \text { Strongly Agree }\end{array}$

I am disappointed in myself for being an offender

$\begin{array}{cccc}1 & 2 & 3 & 4 \\ \text { Strongly Disagree } & \text { Disagree } & \text { Agree } & \text { Strongly Agree }\end{array}$

I feel inferior to people who are not offenders themselves

$\begin{array}{cccc}1 & 2 & 3 & 4 \\ \text { Strongly Disagree } & \text { Disagree } & \text { Agree } & \text { Strongly Agree }\end{array}$

Stereotypes about offenders apply to me

$\begin{array}{cccc}1 & 2 & 3 & 4 \\ \text { Strongly Disagree } & \text { Disagree } & \text { Agree } & \text { Strongly Agree }\end{array}$

People can tell I'm an offender by the way that I look

$\begin{array}{cccc}1 & 2 & 3 & 4 \\ \text { Strongly Disagree } & \text { Disagree } & \text { Agree } & \text { Strongly Agree }\end{array}$


Others think that I cannot achieve much in life because I' $m$ an offender
Strongly Disagree
2
Disagree
3
4
Agree Strongly Agree

People ignore me or take me less seriously just because I'm an offender
1
2
Disagree
3
4
Strongly Disagree
Agree
Strongly Agree

People often treat me like a child just because I'm an offender

$\begin{array}{cccc}1 & 2 & 3 & 4 \\ \text { Strongly Disagree } & \text { Disagree } & \text { Agree } & \text { Strongly Agree }\end{array}$

Nobody would be interested in getting close to me because I'm an offender
1
Strongly Disagree
2
Disagree
3
4

I do not talk much about myself because I do not want to bother people by being an offender
1
Strongly Disagree
2
Disagree
3
4
Agree
Strongly Agree

I do not socialize as much as I used to because being an offender makes me weird
1
Strongly Disagree
2
Disagree
3
Agree
4
Strongly Agree

Negative stereotypes about being an offender keep me away from the normal world

$\begin{array}{cccc}1 & 2 & 3 & 4 \\ \text { Strongly Disagree } & \text { Disagree } & \text { Agree } & \text { Strongly Agree }\end{array}$

I stay away from social situations so I can protect my family and friends from embarrassment

$\begin{array}{cccc}1 & 2 & 3 & 4 \\ \text { Strongly Disagree } & \text { Disagree } & \text { Agree } & \text { Strongly Agree }\end{array}$

Being around people who are not offenders themselves makes me feel out of place or inadequate
1
2
3
4
Strongly Disagree
Disagree
Agree
Strongly Agree 
I avoid getting close to people who are not offenders themselves so I can avoid rejection

$\begin{array}{cccc}1 & 2 & 3 & 4 \\ \text { Strongly Disagree } & \text { Disagree } & \text { Agree } & \text { Strongly Agree }\end{array}$

Offenders are violent people

$\begin{array}{cccc}1 & 2 & 3 & 4 \\ \text { Strongly Disagree } & \text { Disagree } & \text { Agree } & \text { Strongly Agree }\end{array}$

I can have a good and fulfilling life despite being an offender

$\begin{array}{cccc}1 & 2 & 3 & 4 \\ \text { Strongly Disagree } & \text { Disagree } & \text { Agree } & \text { Strongly Agree }\end{array}$

Offenders can make important contributions to society

$\begin{array}{cccc}1 & 2 & 3 & 4 \\ \text { Strongly Disagree } & \text { Disagree } & \text { Agree } & \text { Strongly Agree }\end{array}$

I need other people to make most decisions for me because I'm an offender
Strongly Disagree
2
Disagree
3
4
Agree Strongly Agree

Offenders should not have romantic relationships
1
Strongly Disagree
2
Disagree
3
Agree
4
Strongly Agree

Being an offender has made me a tough survivor
Strongly Disagree
2
Disagree
3
Agree
4
Strongly Agree

I cannot contribute anything to society because I'm an offender
1
Strongly Disagree 2
Disagree
3
Agree
4
Strongly Agree

I feel comfortable being seen in public with other people who are offenders themselves
1 2
Strongly Disagree
Disagree
3
4
Agree
Strongly Agree 
People discriminate against me because I'm an offender

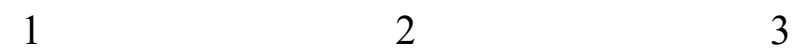

Strongly Disagree Disagree Agree

In general, I am able to live life the way I want to

123

Strongly Disagree $\quad$ Disagree $\quad$ Agree

Offenders cannot live good and rewarding lives

Strongly Disagree

2

Disagree

3

Agree
4

Strongly Agree

4

Strongly Agree 


\section{Appendix O}

\section{Combined Interview Protocol- Revised Adolescent}

Thank you again for agreeing to answer the interview portion of this study. I know we already went through the study information form together, and that you signed and agreed to talk to me; however, I just want to highlight once more that if you tell me about any incidents of abuse regarding a minor I legally need to report it.

Anything you indicated in your questionnaire is fine, because those answers are completely anonymous. There is absolutely no way for myself or anyone else to know what you answered. But because we are sitting face-to-face right now, anything you tell me about experiences you have had personally, or the experiences of someone you know, I will have to report. That includes any sexual behavior between a minor, (so someone under 18 years old), and a professional like a doctor or a therapist-or even a teacher. Also, if you tell me you want to hurt yourself or you want to hurt someone else, I need to report that as well.

I'm not saying that you cannot tell me about those things. You just need to know that I cannot, no matter what, keep it a secret.

That being said, none of the questions I want to ask you right now have anything to do with that. I mostly just want to know a little bit more about you, your family, and your daily life.

\section{DOMAIN 1: PERCEPTIONS OF ASSESSMENT}

1. Can you please describe yourself for the tape?

\section{FOLLOW UP:}

$=$ what is your personality like?

$=$ what are some things you like to do?

2. Can you tell me how you ended up participating in this study, meaning how did you end up at the Royal/CAMH?

NOTE: you can use the answer of this question to lead you into the following one.

\section{Can you describe what the purpose of your assessment today was?}

\section{FOLLOW UP:}

$=$ did anyone tell you what the assessment would be about before you got here?

Parents, lawyer, worker, judge?

= how do you feel about the assessment?

$=$ do you think the assessment will help you?

$=$ why or why not? 


\section{DOMAIN 2: OFFENCE DETAILS}

So, the reason you were referred to the Family Court/Youth Justice Clinic is because you were charged with committing a crime. I know we've already gone through some of the details already - but for the tape....

\section{Could you tell me in your own words what happened?}

NOTE: You can use the summary sheet as a guide to prompt your questions here.

\section{FOLLOW-UP:}

$=$ how did you end up arrested?

= how did you feel when you were arrested?

$=$ who bailed you out?

$=$ how did the police treat you?

NOTE: we are trying to capture the following key information:

- what led up to the offence,

- what actually happened (i.e., level of violence, nature of sexual offence, co-accused, etc.), and;

- how they feel about it in terms of the offence itself AND the process

\section{Were you on substances at the time of your offence? Drugs or alcohol?}

\section{If YES:}

= what specific substances were they on?

$=$ do they regularly use these substances?

$=$ when/age they first started using substances?

= how did they first using substances?

\section{If NO:}

$=$ do you ever use substances?

= if YES: how regularly, age they started, and how they started

\section{What was your state of mind at the time of your offence?}

\section{FOLLOW UP:}

= were you angry? upset?

$=$ did you feel out of control when you were doing it?

$=$ do you remember the offence well? Is it a blurr? 


\section{How do you feel about your offence?}

\section{FOLLOW UP:}

$=$ do you regret what you did? feel guilty?

= how do you cope with what you did?

NOTE: I always preface these questions saying that they don't HAVE to feel bad or guilty - it doesn't matter - we are just curious....don't want to make them feel like they need to feel remorseful

\section{Where were you living at the time of your offence?}

NOTE: If they were not living at home, we want to track ALL of their housing transitions BEFORE the offence, and all transitions SINCE the offence as well.

\section{FOLLOW UP:}

$=$ who were you living with?

Okay - so moving on from the offence, I want to switch gears and ask you some questions about your family.

\section{DOMAIN 3: FAMILY}

9. Can you describe who is in your family?

\section{FOLLOW UP:}

= who was living with you as you were growing up? Siblings? Grandparents? NOTE: If they discuss step-parents, step-children, etc.... you want to determine when these people ENTERED their lives and LEFT.

NOTE: Need to distinguish between biologically-related and non-biologically related family.

NOTE: if they are in the care of CAS, ask them why they were removed from their home, when that happened, where they went. You can use the answer to the previous question as a guide.

\section{Can you describe what your mom is like?}

\section{FOLLOW-UP:}

$=$ what was your mom like growing up?

= what types of things did you typically do together?

= what was your relationship with your mom like growing up? Were you close? 


\section{How did your mom react when she found out about your offence?}

\section{FOLLOW-UP:}

= how did your mom found out you were arrested?

$=$ how did her reaction make you feel?

$=$ did it upset you that she was upset?

= has your relationship with your mom changed since being arrested?

12. Can you describe what your dad is like?

\section{FOLLOW-UP:}

$=$ what was your dad like growing up?

= what types of things did you typically do together?

= what was your relationship with your dad like growing up? Were you close?

13. How did your dad react when he found out about your offence?

\section{FOLLOW-UP:}

= how did your dad found out you were arrested?

$=$ how did their reaction make you feel?

$=$ did it upset you that he was upset?

$=$ has your relationship with your dad changed since being arrested?

NOTE: Often dads will have been in-and-out of the youth's life - need to determine that last time they lived with them, and how it affected them that they left.

\section{How did your parents get along with one another when you were growing up?}

NOTE: you will often get a sense of whether there was domestic abuse in the home...so use that to ask directly about violence in the household.

\section{FOLLOW-UP:}

$=$ did your parents fight a lot? Were they eve physical with each other?

15. Can you describe what your sibling(s) is like?

\section{FOLLOW-UP:}

= what was your relationship like with your sibling(s) growing up? Were you close?

$=$ why do you think you are/are not close with your sibling?

$=$ did you ever have to take care of your sibling(s)? 
16. How did your sibling(s) react when they found out about your offence?

\section{FOLLOW-UP:}

= how did your sibling(s) found out you were arrested?

$=$ how did their reaction make you feel?

$=$ did it upset you that he was upset?

$=$ has your relationship with your sibling(s) changed since being arrested?

17. Can you describe some of the things in your life that bothered you before you were arrested?

\section{FOLLOW UP:}

$=$ anything bother you at school? With friends? Family-stuff?

$=$ why did these things bother you?

= how did these things makes you feel?

18. Would you talk to your parents about the things that bothered you?

\section{FOLLOW UP:}

$=$ why or why not?

$=$ is there anyone you would have talked to? A teacher? Friend?

$=$ does it bother you that you had no one to talk to?

19. Can you tell me what you know about sex?

\section{FOLLOW UP:}

= how would you describe sex to someone who asked you?

\section{Where did you learn about sex?}

NOTE: they may have learned about sex on the internet - you want to specify if they mean porn. If YES, then ask them when and why they first started seeking out porn.

\section{FOLLOW-UP:}

$=$ what types of things did you learn? 
21. Did your parents ever have "the talk" with you?

\section{FOLLOW UP:}

$=$ do you remember what they told you?

$=$ do you know how old you were when they did that?

22. Do you ever talk about sex with your parents?

\section{FOLLOW UP:}

$=$ why or why not?

= if you had questions about sex, who would you ask? What would you do?

$=$ do you feel like you have a healthy sexuality?

\section{DOMAIN 4: RELATIONSHIPS}

23. Can you tell me about any other family members involved in your life?

\section{FOLLOW UP:}

$=$ do they know about your offence?

$=$ why or why not?

$=$ has your relationship with them changed since being arrested?

$=$ How so?

$=$ How does it make you feel?

$=$ Does it have to do with the type of offence you've been charged with?

$=$ do you generally feel supported by your family

\section{Can you describe who your friends are?}

\section{FOLLOW UP:}

= how many close friends would you say you have?

$=$ male or female?

$=$ do they know about your offence?

= why or why not? 
25. Has your relationship with your friends changed since being arrested?

\section{FOLLOW UP:}

= how so?

$=$ how does it make you feel?

$=$ does it have to do with the type of offence you've been charged with?

NOTE: if they don't have any friends, I always ask them if they feel lonely.

26. How do you feel around your friends since you were arrested?

\section{DOMAIN 5: MENTAL HEALTH}

27. Can you describe any experience you have had with the mental health system?

FOLLOW UP:

$=$ have you ever been tested at school?

$=$ have you ever been given a diagnosis?

$=$ if YES: what diagnoses? When were they given?

$=$ are you on any medication?

28. How do you feel about coming to this clinic for a mental health assessment?

29. Do you think your mental health has been affected since being charged?

\section{FOLLOW UP:}

$=$ do you feel more depressed or anxious?

$=$ have you noticed changes in your sleep? Anything like that....?

\section{FOLLOW UP:}

$=$ In what ways has your mental health been affected?

$=$ Is there anyone you talk to?

$=$ Why or why not?

$=$ Do you wish you had access to treatment?

30. Can you describe some of the ways you cope with going through the court process? FOLLOW UP:

$=$ is there anything you do to help you relax? Exercise? Substances? Self-harming? 


\section{DOMAIN 6: SCHOOL}

31. Can you describe what your relationship with school is like?

\section{FOLLOW UP:}

$=$ do you like school?

$=$ are you a good student?

= were you ever suspended or expelled before you were charged?

32. Are you in the same school you were in when you were charged?

\section{FOLLOW UP:}

= why or why not?

= how did it make you feel to leave?

$=$ do you think it is fair that you had to leave? Why or why not?

NOTE: We want to document ALL school-related transitions since being charged and the reasons WHY they transitioned.

33. Do the people at your school know about your charges?

\section{FOLLOW UP:}

= why or why not?

$=$ does it have to do with the type of offence you've been charged with?

34. How do you feel at school since being charged?

\section{FOLLOW UP:}

$=$ do you feel differently at school? Why or why not?

\section{DOMAIN 7: CRIMINAL JUSTICE SYSTEM}

35. Can you tell me how you feel about going to court? Specifically, how do you feel when you are actually at court?

\section{FOLLOW UP:}

$=$ Do you think you are treated differently at court because of your race? LGBTQ status? Native/Indigenous status? 
36. Do you have any court-ordered conditions?

\section{FOLLOW UP:}

= can you describe how your conditions have affected your life?

$=$ do you think your conditions are appropriate/fair? Why or why not?

37. Do you have a legal aid lawyer?

\section{FOLLOW UP:}

$=$ do you like them?

$=$ did they encourage you to plead guilty (based on summary sheet)

38. Since being arrested have you ever been placed in custody?

\section{FOLLOW UP:}

$=$ why do you think you were sent to custody?

$=$ how do you feel when in custody?

39. How do you feel about the police?

NOTE: you can revisit experiences with the police from earlier on in this section.

NOTE: If applicable: How do you feel about CAS, group homes, etc.?

\section{DOMAIN 8: PERCEPTIONS OF THE FUTURE}

40. How do you feel about your future?

41. Do you think being labelled as someone who has committed an offence will affect your future?

\section{FOLLOW UP:}

$=$ why or why not?

$=$ do you think your feelings have to do with the type of offence you've been charged with?

$=$ IF they discuss the benefits of having a youth record, or sealed record, ask they

HOW they know about this? Who told them?

NOTE: we want to know their thought process behind their feelings re: future - where did they pick up the assumptions they hold about their futures. 
42. Can you tell me what the worst part of going through this process has been for you? Specifically, from the time you were charged to now, what has been the worse part?

\section{FOLLOW UP:}

$=$ why is that the worst part?

43. Can you tell me what the best part of going through this process has been for you, if there has been one?

\section{FOLLOW UP}

$=$ why is this the best part?

44. If there was one thing about you that you wanted the world to know, or if you had one piece of advice to offer to someone else in your position, what would it be? 